\title{
A Rapid Dosimetric Assessment Method Using Cone Beam CT in Prostate Cancer Patients
}

\author{
Almatani, Turki U.
}

How to cite:

Almatani, Turki U. (2017) A Rapid Dosimetric Assessment Method Using Cone Beam CT in Prostate Cancer Patients. Doctoral thesis, Swansea University.

http://cronfa.swan.ac.uk/Record/cronfa50330

Use policy:

This item is brought to you by Swansea University. Any person downloading material is agreeing to abide by the terms of the repository licence: copies of full text items may be used or reproduced in any format or medium, without prior permission for personal research or study, educational or non-commercial purposes only. The copyright for any work remains with the original author unless otherwise specified. The full-text must not be sold in any format or medium without the formal permission of the copyright holder. Permission for multiple reproductions should be obtained from the original author.

Authors are personally responsible for adhering to copyright and publisher restrictions when uploading content to the repository.

Please link to the metadata record in the Swansea University repository, Cronfa (link given in the citation reference above.)

http://www.swansea.ac.uk/library/researchsupport/ris-support/ 


\title{
A Rapid Dosimetric Assessment Method Using Cone Beam CT in Prostate Cancer Patients
}

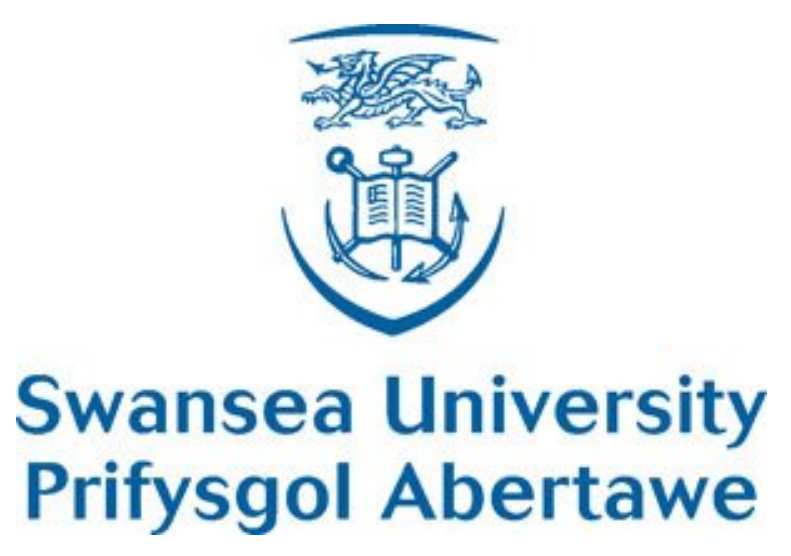

\section{Turki Uthman Almatani}

Submitted to Swansea University in fulfilment of the requirements for the

Degree of Doctor of Philosophy

\author{
School of Medicine
}

Swansea University 2017 


\section{Abstract}

Objective: Cone beam CT (CBCT) images contain more scatter than a conventional CT image and therefore provide inaccurate Hounsfield units (HU). Consequently CBCT images cannot be used directly for radiotherapy dose calculation. The aim of this study is to enable dose calculations to be performed with the use of CBCT images taken during radiotherapy and evaluate the necessity of re-planning.

Methods: A phantom, a standard prostate cancer patient and prostate cancer patients with single and double metallic hips were imaged using both CT and CBCT. A multilevel threshold algorithm (MLT) was used to categorise pixel values in the CBCT images into segments of homogeneous HU. The variation in HU with position in the CBCT images was taken into consideration and the benefit of using a large number of materials has been explored. This segmentation method relies upon the operator dividing the CBCT data into a set of volumes where the variation in the relationship between pixel values and HUs is small. In addition, an automated MLT algorithm was developed to reduce the operator time associated with the process. Furthermore, magnetic resonance (MR) images of the standard prostate case were segmented and converted into HUs using the MLT algorithm. Radiotherapy treatment plans were generated from CT images and then copied to the segmented CBCT and MR data sets and the doses were recalculated and compared using pencil beam (PB), collapsed cone (CC) and Monte Carlo (MC) algorithms.

Results: Compared with the planning CT (pCT) treatment plan, in the phantom case, a gamma evaluation showed all points in planning target volume (PTV), rectum and bladder had gamma value $<1(3 \% / 3 \mathrm{~mm})$ in the segmented CBCT, when considering only 2 material bins, water and bone. For the standard patient case, using 3 materials, air, water and bone, was accurate enough to provide accurate dose calculations with differences of less than $2 \%$. For the patient with a metallic hip, increasing the number of bins to define the material type from 7 materials to 8 materials, required $50 \%$ more operator time to improve the accuracy by $0.01 \%$ using PB and CC and $0.05 \%$ when using $\mathrm{MC}$ algorithms. The use of 5 values of $\mathrm{HU}$ (air, adipose, water, bone and metal implant) gave the best balance between dose accuracy and operator time (3.5 hours). For the patient with double hip prosthetics, segmenting CBCT into 5 materials with the MLT algorithm showed $-0.46 \%$ dose difference with 8 hours operator time, whilst the automated MLT algorithm showed $-1.36 \%$. For the standard case, the segmentation of MR images, into 3 materials, resulted in a dose difference of $-1.31 \%$ with 2 hours operator time.

Conclusion: The segmentation of CBCT images using the method in this study can be used for dose calculation. For a simple phantom and standard prostate case, 2 and 3 values of HU were needed to improve dose calculation accuracy, respectively. For patients with additional anatomical inhomogeneities such as metallic hips, 5 values of $\mathrm{HU}$ were found to be needed, giving a reasonable balance between dose accuracy and operator time. The automated MLT algorithm reduced the operator time associated with implementing the MLT algorithm to achieve clinically acceptable accuracy. This saved time makes the automated MLT algorithm superior and easier to implement in the clinical setting. The MLT method can be applicable for the dose calculation on MR images and can be of interest to MRI-only based radiotherapy treatment planning. 


\section{Table of Contents}

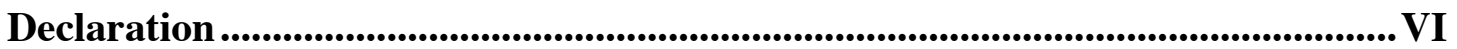

Acknowledgment .............................................................................................................. VII

List of Figures ...................................................................................................... VIII

List of Tables ..............................................................................................

List of Abbreviations....................................................................................

List of publications and academic conferences ...................................................XVII

Chapter 1 Introduction ........................................................................................................... 1

1.1 Introduction and background ................................................................................ 2

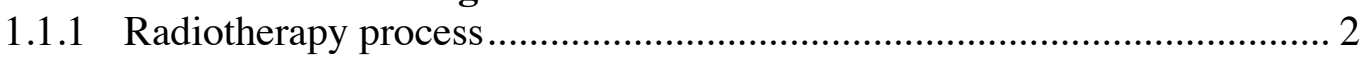

1.1.2 Image guided radiotherapy …………………………………………... 7

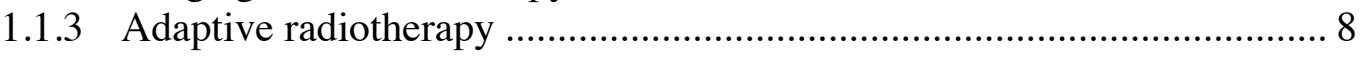

1.1.4 Cone beam computed tomography …………………………………...... 10

1.2 Literature review and previous work ............................................................... 11

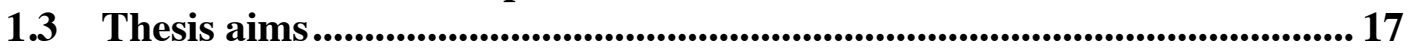

1.4 Thesis structure ................................................................................... 20

Chapter 2 Cone beam computed tomography .......................................................... 22

2.1 Introduction .................................................................................................. 23

2.2 XVI system .................................................................................................................. 23

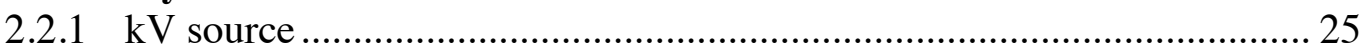

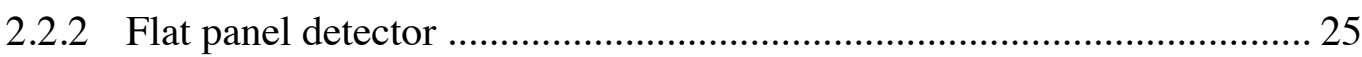

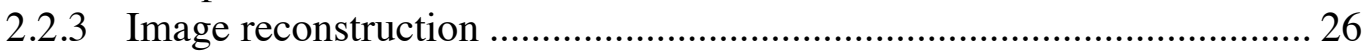

2.3 CBCT in image-guided radiotherapy for prostate cancer ......................... 27

2.4 CBCT in adaptive radiotherapy for prostate cancer................................... 31

2.5 CBCT image quality ............................................................................................... 32

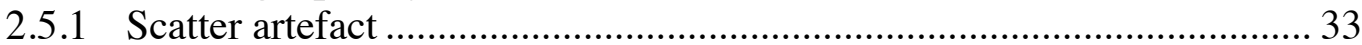

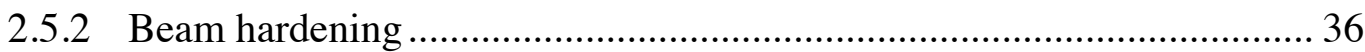

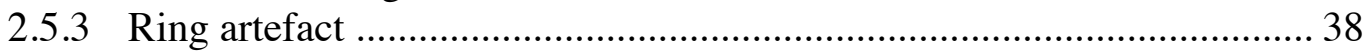

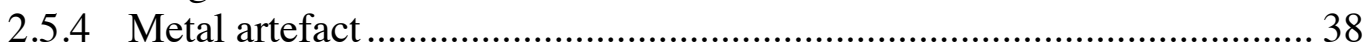

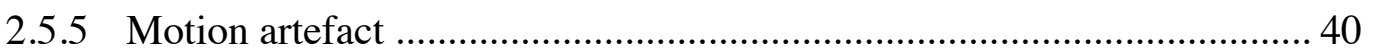

2.5.6 Cone-beam and truncation artefact....................................................... 42

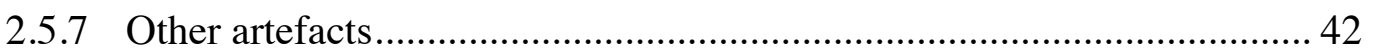

Chapter 3 Dose calculation algorithms.......................................................... 45

3.1 Introduction ......................................................................................................... 46

3.2 Effective path length method ............................................................................. 47

3.3 Equivalent tissue-air method ........................................................................... 48

3.4 Convolution/Superposition method ................................................................5 50

3.5 Monte Carlo method ....................................................................................56

Chapter 4 The Monte Carlo simulation .............................................................59

4.1 Introduction ...........................................................................................................6 60

4.2 Monte Carlo radiation transport ................................................................6 61

4.3 Photon transport ..................................................................................................... 62

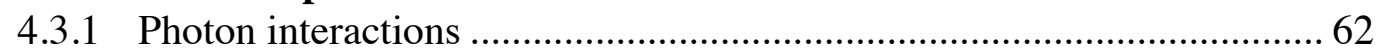

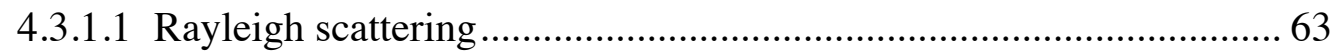




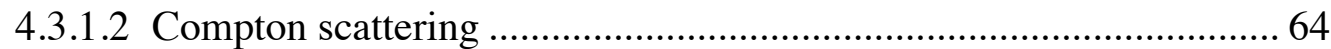

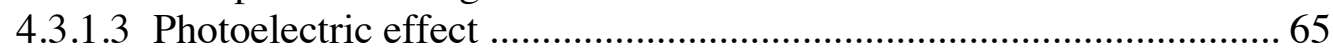

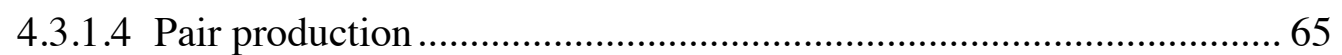

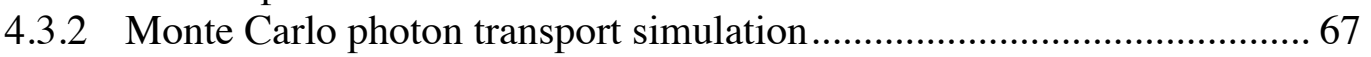

4.4 Electron transport ............................................................................69

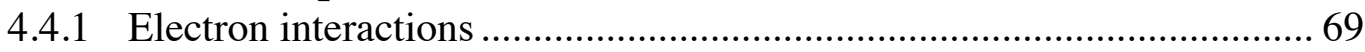

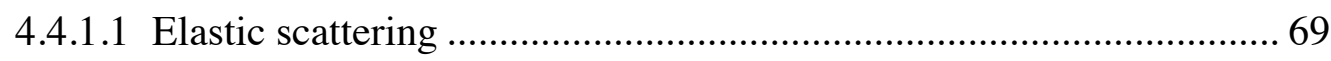

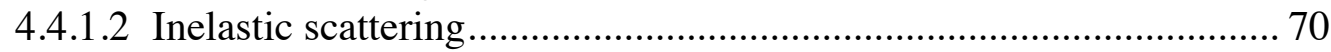

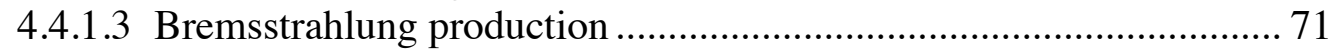

4.4.2 Monte Carlo electron transport simulation ........................................... 72

4.5 EGSnrc Monte Carlo code ........................................................................ 75

Chapter 5 Monte Carlo modelling of the linear accelerator head ....................... 77

5.1 Introduction ..............................................................................................78

5.2 Materials and methods ........................................................................... 78

5.2.1 Tuning and validation of the Linac model......................................... 78

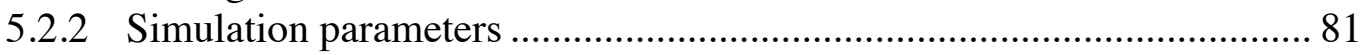

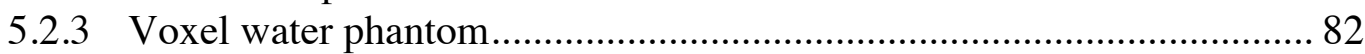

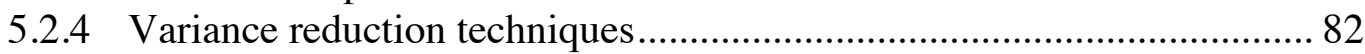

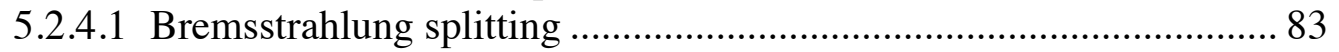

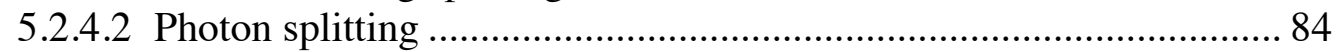

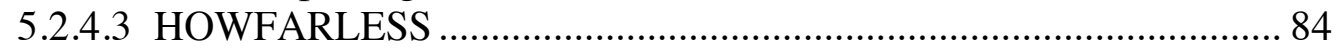

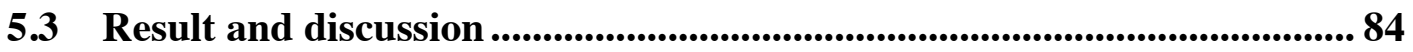

5.3.1 PDD and dose profile comparison .................................................. 84

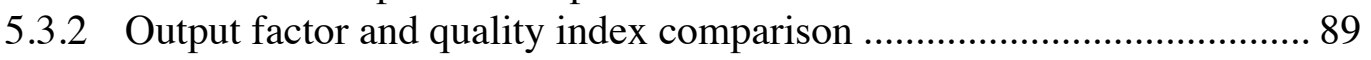

5.4 Conclusion ..............................................................................................90 90

Chapter 6 CBCT-based dose calculation of a male pelvis phantom .................. 91

6.1 Introduction ..................................................................................................... 92

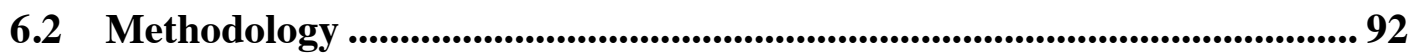

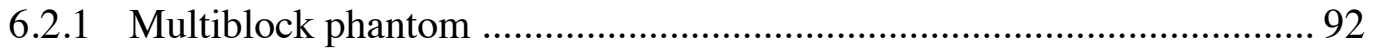

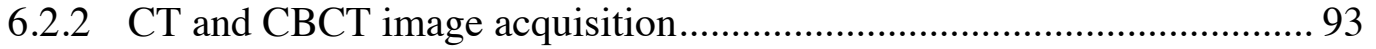

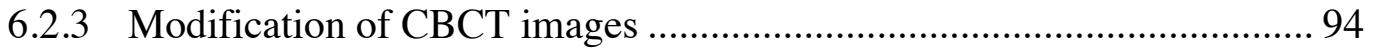

6.2.4 Treatment planning system and treatment plan ..................................... 96

6.2.5 Monte Carlo-based treatment plan ..................................................... 97

6.2.6 Conversion of CT images into MC format .......................................... 98

6.2.7 Treatment planning evaluation and comparison................................ 100

6.2.7.1 DVH and dose profile comparison.............................................. 100

6.2.7.2 Gamma index comparison ........................................................ 100

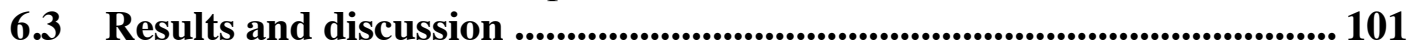

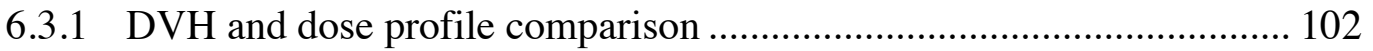

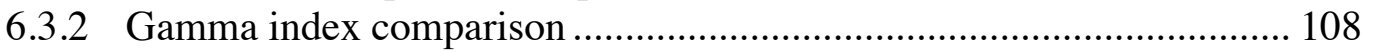

6.4 Conclusion ............................................................................................................ 109

Chapter 7 CBCT-based dose calculation of a prostate cancer patient ............. 110

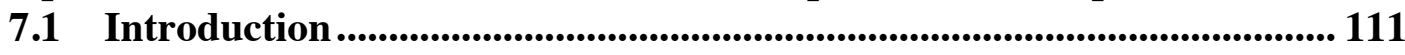

7.2 Methodology .................................................................................. 111

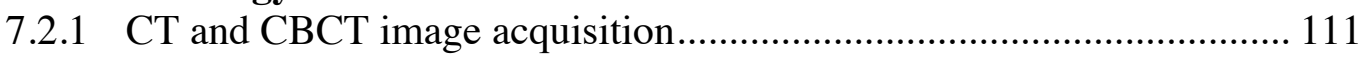

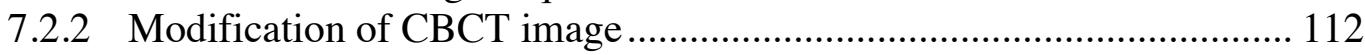

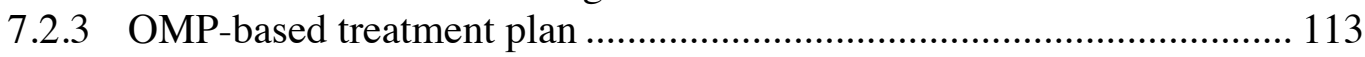

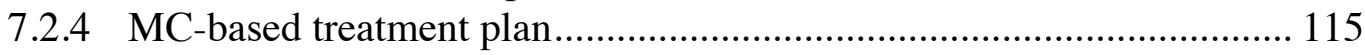

7.2.5 Treatment planning evaluation and comparison................................ 117 


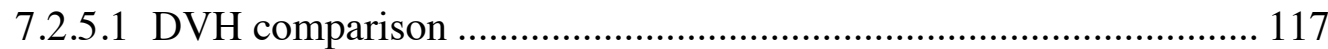

7.2.5.2 Dose profile comparison ................................................................ 117

7.2.5.3 Gamma index comparison ............................................................. 117

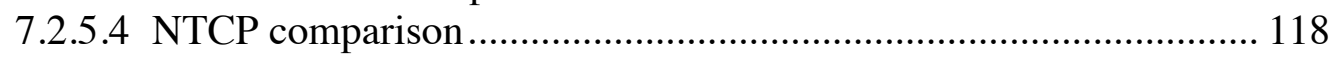

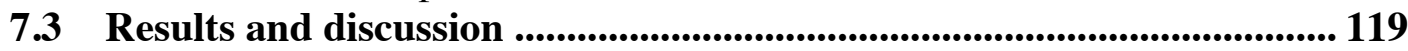

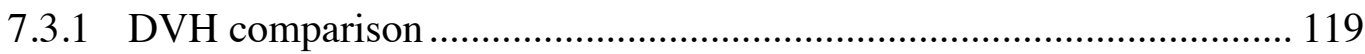

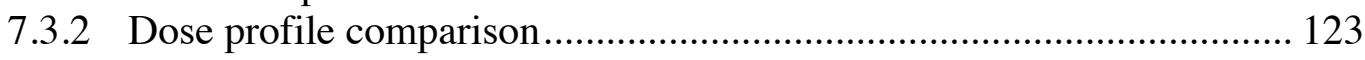

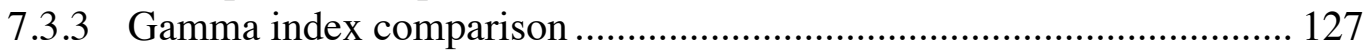

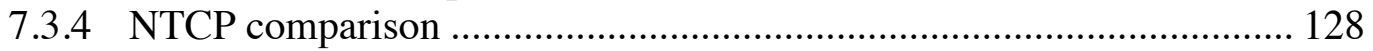

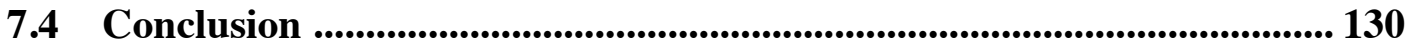

Chapter 8 CBCT-based dose calculation of a prostate cancer patient with single hip prosthesis .................................................................................................................... 131

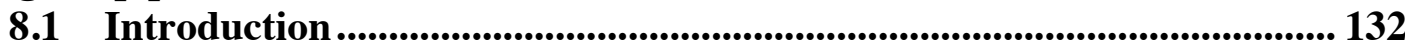

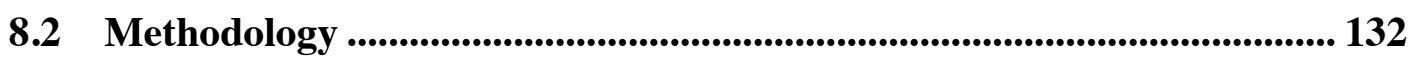

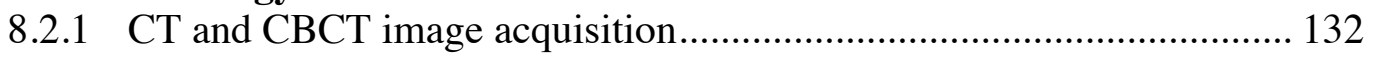

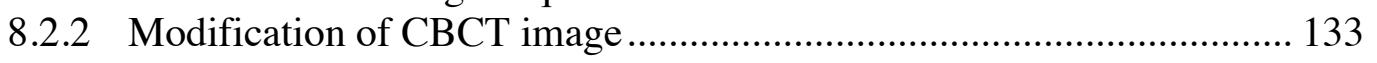

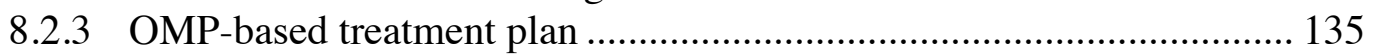

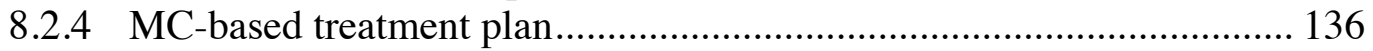

8.2.5 Treatment planning evaluation and comparison................................. 136

8.2.5.1 DVH and dose profile comparison.............................................. 137

8.2.5.2 Gamma and conformity index comparison .................................... 137

8.2.5.3 Dose point comparison............................................................... 137

8.3 Result and discussion ......................................................................... 138

8.3.1 Dose profile and DVH comparison ................................................. 138

8.3.2 Gamma and conformity index comparison ...................................... 143

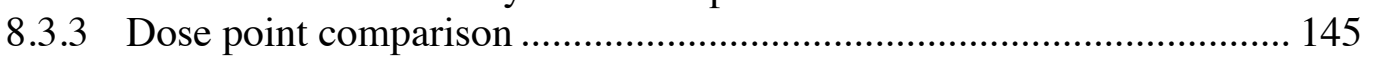

8.4 Conclusion .................................................................................... 146

Chapter 9 CBCT-based dose calculation of a prostate cancer patient with double hip prostheses ............................................................................................................. 147

9.1 Introduction ............................................................................................................ 148

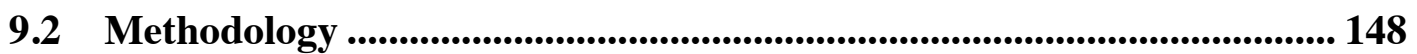

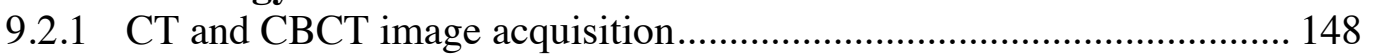

9.2.2 Modification of CBCT image ......................................................... 149

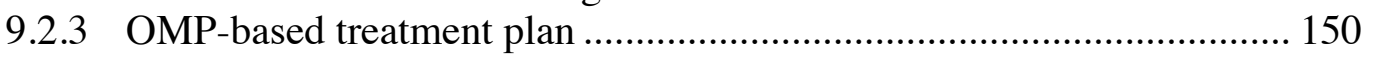

9.2.4 MC-based treatment plan ............................................................... 150

9.2.5 Treatment planning evaluation and comparison................................. 151

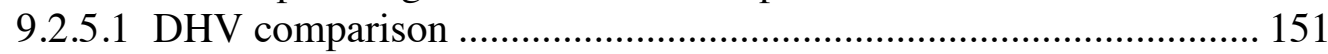

9.2.5.2 Hip and bone volume comparison ............................................. 151

9.2.5.3 Gamma and conformity index comparison .................................. 151

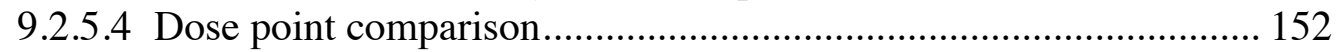

9.3 Result and discussion ................................................................................... 152

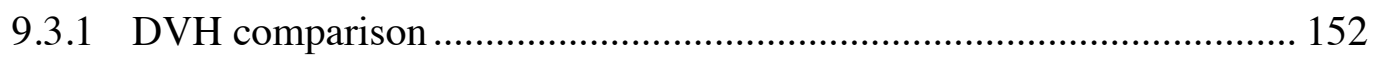

9.3.2 Hip and bone volume comparison .................................................. 155

9.3.3 Gamma and conformity index comparison ........................................ 156

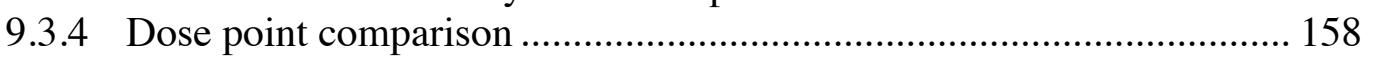

9.4 Conclusion .......................................................................................... 159

Chapter 10 Automated algorithm for CBCT-based dose calculations of a prostate radiotherapy ..................................................................................................... 160

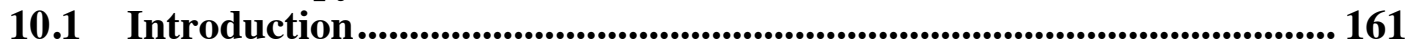




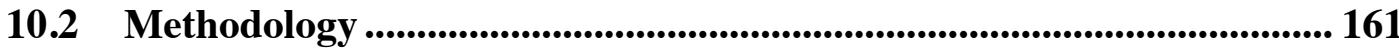

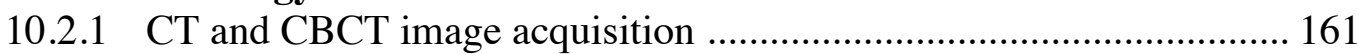

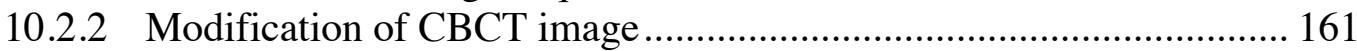

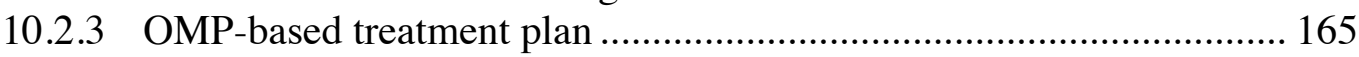

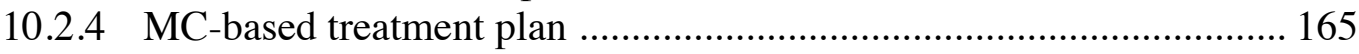

10.2.5 Treatment planning evaluation and comparison ................................ 166

10.2.5.1 Dose profile comparison ............................................................ 167

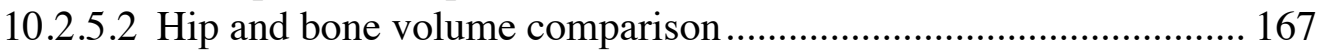

10.2.5.3 Gamma and conformity index comparison ................................... 167

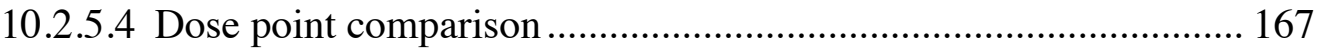

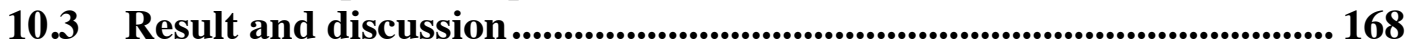

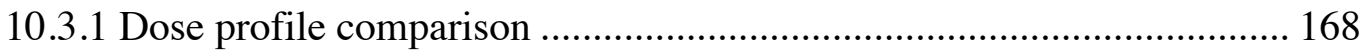

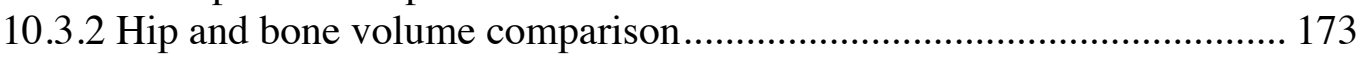

10.3.3 Gamma and conformity index comparison ......................................... 175

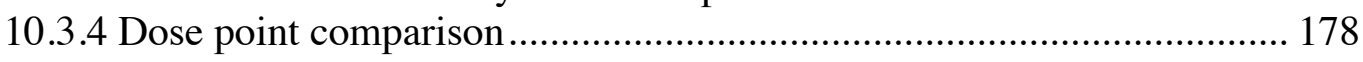

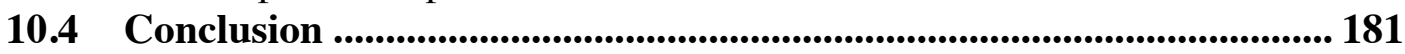

Chapter 11 MR-based dose calculation of a prostate cancer patient .............. 182

11.1 Introduction ........................................................................................................ 183

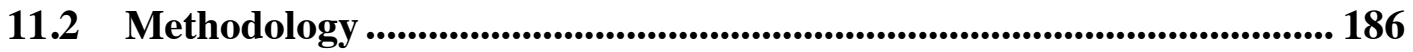

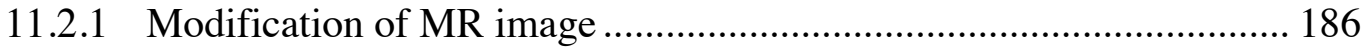

11.2.2 Treatment planning evaluation and comparison ............................... 188

11.3 Results and discussion ............................................................................ 188

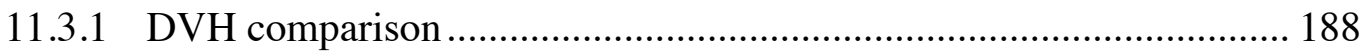

11.3.2 Gamma index comparison ............................................................ 191

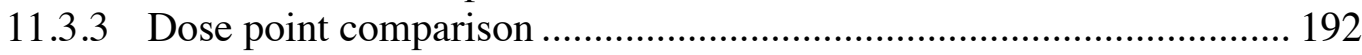

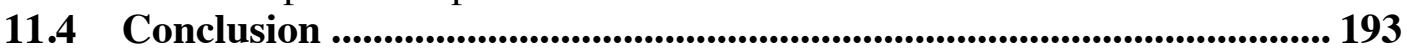

Chapter 12 Conclusion and future work .............................................................. 194

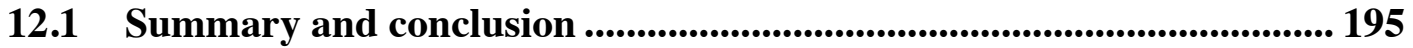

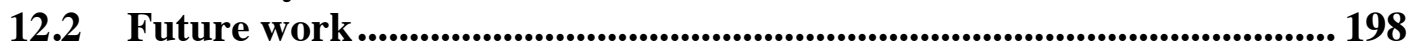

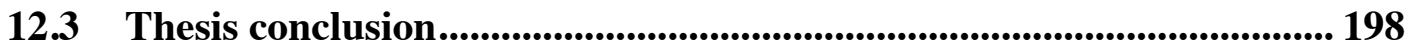

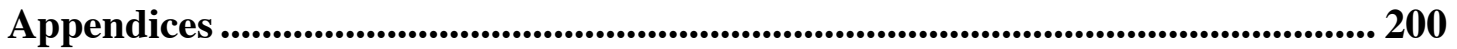

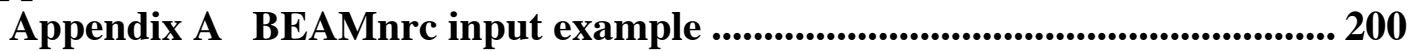

Appendix B DOSXYZnrc input example.......................................................... 206

Appendix C MLT automated algorithm example .............................................. 207

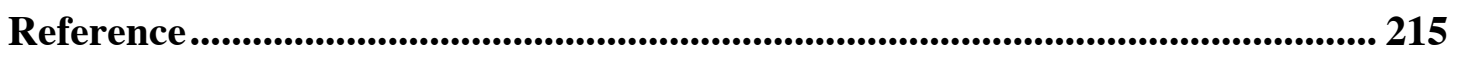




\section{Declaration}

This work has not previously been accepted in substance for any degree and is not being concurrently submitted in candidature for any degree.

Signed (candidate)

Date

\section{STATEMENT 1}

This thesis is the result of my own investigations, except where otherwise stated. Where correction services have been used, the extent and nature of the correction is clearly marked in a footnote(s).

Other sources are acknowledged by footnotes giving explicit references. A bibliography is appended.

Signed (candidate)

Date

\section{STATEMENT 2}

I hereby give consent for my thesis, if accepted, to be available for photocopying and for inter-library loan, and for the title and summary to be made available to outside organisations.

Signed (candidate)

Date 


\section{Acknowledgment}

This thesis would not have been possible without the support of many people. I would like to owe many thanks to my supervisors Dr. Richard Hugtenburg, Dr. Ryan Lewis, Dr. Susan Barley and Mr. Mark Edwards for their support and assistance. It has been a privilege to have supervisors who always keep the door open and are eagerly willing to provide me with invaluable advice, unending assistance and a level of flexibility that I truly appreciate.

I would like to thanks the staff of the Department of Medical Physics and Clinical Engineering in Singleton Hospital, ABMU, especially Mr. James Williams, Mr. Adam Selby and Mr. Douglas Etheridge for their assistance in this study.

I gratefully acknowledge Umm al-Qura University and The Ministry of Higher Education of Saudi Arabia for providing me this sponsorship.

Finally, I wish to express my love and gratitude to my beloved family, especially my mother Salwa, my father Uthman, my sisters, Fay and Jonah, and my brothers, Nawaf and Mohammad without their love and support I would never have come to this point. 


\section{List of Figures}

Figure 1.1: Typical calibration curve between CT number and electron density (Khan 3

Figure 1.2: A tumour with different volumes ................................................... 5

Figure 1.3: Elekta Synergy linear accelerator at Singleton Hospital .......................... 9

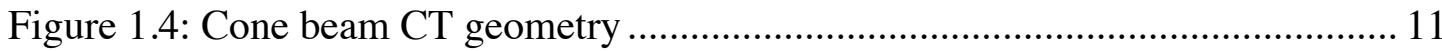

Figure 1.5: Current verification process using CBCT image ................................... 18

Figure 1.6: Verification process using modified/corrected CBCT image................... 19

Figure 2.1: Construction of flat panel detector, 1 Aluminium, 2 air gap, 3 CsI scintillator, 4 attenuator and 5 photodiodes (Courtesy of Elekta) (Giles, 2010)........ 26

Figure 2.2: Example image illustrating X-ray scatter artefacts, due the presence of high density material, hip prosthesis

Figure 2.3: Example images illustrating beam hardening, between highly attenuating materials, radar and scatter artefacts 37

Figure 2.4: Example images illustrating metal artefacts due to the appearance of single (left) and double hip (right) prostheses

Figure 2.5: Example images illustrating motion artefacts 41

Figure 3.1: Convolution method performed with TERMA and kernel to obtain dose distribution (Papanikolaou et al., 2004) 51

Figure 3.2: An example of CC, 13 cone axes representing 26 possible directions of particle transport cover a lattice made of $3 \times 3 \times 3$ voxels (Ahnesjö, 1989)

Figure 4.1: The estimation of the value of $\pi$ using the MC method. Left panel: shows the estimated value of $\pi, 3.208$, using 1000 events. Right panel: shows the estimated value of $\pi, 3.146$, using 10000 events

Figure 4.2: The cross-sections of different photon interactions $\left(\mathrm{cm}^{2}\right)$ against energy $(\mathrm{eV})$ for water (generated using the PENELOPE MC code) 63

Figure 4.3: The photon mean free path to different photon interactions $(\mathrm{cm})$ against energy $(\mathrm{eV})$ in water (generated using the PENELOPE MC code) 66

Figure 4.4: The cross-sections of different electron interactions $\left(\mathrm{cm}^{2}\right)$ against energy $(\mathrm{eV})$ for water (generated using the PENELOPE MC code) 70

Figure 4.5: The electron mean free path and range $\left(\mathrm{g} / \mathrm{cm}^{2}\right)$ against energy $(\mathrm{eV})$ in water (generated using the PENELOPE MC code) .73

Figure 5.1: A view of the Linac $6 \mathrm{MV}$ model in (a) the xz and (b) the yz plane 79 
Figure 5.2: Comparison between measured PDD data of the $6 \mathrm{MV}$ beam (red solid line) and simulated PDD for $3 \times 3,5 \times 5,7 \times 7$ and $10 \times 10 \mathrm{~cm}^{2}$ field size at $90 \mathrm{~cm}$ SSD with $6.05 \mathrm{MeV}$.... 85

Figure 5.3: Lateral dose profiles comparisons for open beam between measured (solid line) and simulation (dotted line) of $5 \times 5,10 \times 10,15 \times 15$ and $20 \times 20 \mathrm{~cm}^{2}$ at $1.5,5$, 10 and $20 \mathrm{~cm}$ depths along jaws axis using $6.05 \mathrm{MeV}$ electron energy with $0.14 \mathrm{~cm}$ spot size..... 86

Figure 5.4: Lateral dose profiles comparisons for wedged beam between measured (solid line) and simulation (dotted line) of $5 \times 5,10 \times 10,15 \times 15$ and $20 \times 20 \mathrm{~cm}^{2}$ at $1.5,5,10$ and $20 \mathrm{~cm}$ depths along jaws axis using $6.05 \mathrm{MeV}$ electron energy with $0.14 \mathrm{~cm}$ spot size 87

Figure 5.5: Lateral dose profiles comparisons for wedged beam between measured (solid line) and simulation (dotted line) of $5 \times 5,10 \times 10,15 \times 15$ and $20 \times 20 \mathrm{~cm}^{2}$ at $1.5,5,10$ and $20 \mathrm{~cm}$ depths along MLC axis using $6.05 \mathrm{MeV}$ electron energy with $0.14 \mathrm{~cm}$ spot size 88

Figure 5.6: Output factors comparison between measurement (solid line) and simulation (dotted line) for different field sizes for open and wedged beams .............89

Figure 6.1: Multiblock phantom scan using (a) CBCT, (c) pCT and the resultant image after segmenting CBCT, (b) sCBCT 93

Figure 6.2: Segmentation of CBCT process using the MLT algorithm 95

Figure 6.3: The pCT of the multiblock phantom with the drawn structures 96

Figure 6.4: The CT ramp for the conversion of CT values to material type and density used in OMP. The same ramp was used in MC. The density and composition of the material used in this ramp were included in the PEGS4 cross section data. 98

Figure 6.5: A CT transverse slice of the Multiblock phantom in egsphant format (generated using DOSXYZ_show utility, provided with EGSnrc package 99

Figure 6.6: DVHs comparison between pCT, CBCT and sCBCT plans for PTV, rectum and bladder using (a) PB, (b) CC and (c) MC algorithm (prescription dose 74 Gy). . .104

Figure 6.7: Comparison of the dose profile of pCT, CBCT and sCBCT plans at the isocentre depth using (a) $\mathrm{PB}$, (b) $\mathrm{CC}$ and (c) $\mathrm{MC}$ algorithm. The second y axis represents the CBCT number, sCBCT number and CT number. Subplots represent the differences between $\mathrm{pCT}$ and $\mathrm{SCBCT}$ dose profiles 107

Figure 7.1: A prostate patient scan using (a) CBCT, (c) pCT and the resultant image after segmenting CBCT, (b) sCBCT . .112

Figure 7.2: A screenshot of a prostate IMRT plan using (a) OMP with CC algorithm and (b) MC, both displayed using CERR 115

Figure 7.3: DVHs comparison between pCT and sCBCT plans for PTV, rectum and bladder using (a) PB, (b) CC and (c) MC algorithm (prescription dose 60 Gy) ........121 
Figure 7.4: Comparison of the dose profile of $\mathrm{pCT}$ and $\mathrm{sCBCT}$ plans at the isocentre depth using (a) PB, (b) $\mathrm{CC}$ and (c) MC algorithm. The second y axis represents the sCBCT number and CT number. Subplots represent the differences between pCT and sCBCT dose profiles 125

Figure 7.5: NTCPs for IMRT plan based on pCT and sCBCT when using (a) PB, (b) $\mathrm{CC}$ and (c) MC algorithms

Figure 8.2: Comparison of the dose profile of $\mathrm{pCT}$, sCBCT3 and sCBCT8 plans at the isocentre depth and the absolute difference between sCBCT8 and pCT using (a) PB, $\mathrm{CC}$ and (c) $\mathrm{MC}$ algorithms 128

Figure 8.1: Two different locations and slices of the original CBCT (a and c) and the resultant images after segmentation using the same threshold values in only a part of the image (b and d respectively). In particular it is observed in (d) that the artefacts in the vicinity of the metal are erroneously corrected. Variable threshold values were then used (f) which compared well with the original pCT image (e) .135

Figure 8.2: Comparison of the dose profile of $\mathrm{pCT}, \mathrm{sCBCT} 3$ and $\mathrm{sCBCT} 8$ plans at the isocentre depth and the absolute difference between sCBCT8 and pCT using (a) PB, $\mathrm{CC}$ and (c) MC algorithms 140

Figure 8.3: DVHs comparison pCT (:v), sCBCT8 (solid line), sCBCT5 (--) and sCBCT3 (-.) IMRT plans for PTV95, rectum and bladder using (a) PB, (b) CC and (c) MC algorithms .142

Figure 8.4: Summary of the $\gamma$ index with fixed DTA $=3 \mathrm{~mm}$ and DD $=3 \%$ for the calculation points falling inside the PTV, rectum and bladder, showing the fraction of points resulting with $\gamma<1$. (b) CI comparison between pCT and sCBCT plans using $\mathrm{PB}, \mathrm{CC}$ and $\mathrm{MC}$ algorithms against number of bins .144

Figure 8.5: Dose comparison between $\mathrm{pCT}$ and sCBCT plans at the isocentre against operator time using PB (..), CC (--) and MC (-.) algorithms 145

Figure 9.1: A prostate cancer patient with bilateral hip replacement using (a) pCT and the resultant images after segmentation (b) $1^{\text {st }} \mathrm{sCBCT}$, (c) $2^{\text {nd }} \mathrm{sCBCT}$ and (d) $3^{\text {rd }}$ sCBCT 149

Figure 9.2: DVHs comparison $\mathrm{pCT}, 1^{\text {st }} \mathrm{sCBCT}, 2^{\text {nd }} \mathrm{sCBCT}$ and $3^{\text {rd }} \mathrm{sCBCT}$ IMRT plans for PTV95, rectum and bladder using (a) PB, (b) CC and (c) MC algorithms.154

Figure 9.3: Image volume histogram (IVHs) comparison pCT and $1^{\text {st }} \mathrm{sCBCT}$ for left (LT) and right (RT) hip 155

Figure 9.4: Right/Left hip and bone volume differences between $\mathrm{pCT}$ and $1^{\text {st }} \mathrm{sCBCT}$, $2^{\text {nd }}$ SCBCT and $3^{\text {rd }}$ sCBCT. 156

Figure 9.5: Summary of the $\gamma$ index with fixed DTA $=3 \mathrm{~mm}$ and DD $=3 \%$ for the calculation points falling inside the PTV, rectum and bladder, showing the fraction of points resulting with $\gamma<1$. (b) CI comparison between pCT and sCBCT plans using $\mathrm{PB}, \mathrm{CC}$ and $\mathrm{MC}$ algorithms 157 
Figure 9.6: Dose comparison between $\mathrm{pCT}$ and $\mathrm{sCBCT}$ plans at the isocentre against operator time using $\mathrm{PB}, \mathrm{CC}$ and $\mathrm{MC}$ algorithms

Figure 10.1: A slice of the pCT (a) of the standard case and the original CBCT (b), and the resultant images after segmentation CBCT using the manual MLT (sCBCTman) and the automated MLT (sCBCTauto) (c and d respectively)

Figure 10.2: A slice of the pCT (a) of the single hip prosthesis case and the original CBCT (b), and the resultant images after segmentation CBCT using the manual MLT (sCBCTman) and the automated MLT (sCBCTauto) (c and d respectively) 162

Figure 10.3: A slice of the pCT (a) of the double hip prostheses case and the original CBCT (b), and the resultant images after segmentation CBCT using the manual MLT (sCBCTman) and the automated MLT (sCBCTauto) (c and d respectively) 163

Figure 10.4: Image histogram of the bilateral hip prostheses case in radius number 4 only through all slices (a) and an image histogram of a single slice in radius number 4 (b), showing how the lower threshold values of each material are defined 164

Figure 10.5: Comparison of the dose profiles of pCT, sCBCTman and sCBCTauto plans around the isocentre depth using $\mathrm{MC}$ algorithm for the standard (a), single hip (b) and double hip prostheses cases (c). The second y axis represents the sCBCTman numbers and $\mathrm{sCBCT}$ auto number and $\mathrm{CT}$ number. Subplots represent the differences between $\mathrm{pCT}$ and $\mathrm{sCBCT}$ man/sCBCTauto dose profiles 170

Figure 10.6: Right/Left hip and bone volume differences between $\mathrm{pCT}$ and sCBCTman and sCBCTauto for the (a) standard, (b) single hip and (c) double hip prostheses cases 174

Figure 10.7: A slice of the pCT of the bilateral hip prostheses case (a) and the resultant images after segmentation of CBCT using the manual MLT (sCBCTman) and the automated MLT (sCBCTman) (b and c respectively), showing the HU value difference in the left hip prosthesis 175

Figure 10.8: Dose comparison between pCT, sCBCTman and sCBCTauto plans at the isocentre using $\mathrm{PB}, \mathrm{CC}$ and $\mathrm{MC}$ algorithms for the (a) standard, (b) single hip and (c) double hip prostheses cases 179

Figure 11.1: A prostate patient scan using (a) pCT, (b) MR and the resultant image after segmenting MR, (c) sMR 187

Figure 11.2: DVHs comparison between pCT and SMR plans for PTV, rectum and bladder using (a) PB, (b) CC and (c) MC algorithm (prescription dose $60 \mathrm{~Gy}$ ) 190 


\section{List of Tables}

Table 5.1 The component models and materials of the $6 \mathrm{MV}$ Linac model 80

Table 5.2: The transport parameters used in BEAMnrc and DOSXYZnrc in this study 82

Table 6.1 Element composition of male pelvis phantom tissue substitutes 100

Table 6.2: Dose and coverage differences between CBCT/sCBCT plans and the pCT plan, in \%, for the PTV, rectum and bladder 105

Table 6.3: Summary of the index with fixed DTA $=3 \mathrm{~mm}$ and DD $=3 \%$ for the calculation points falling inside the PTV, rectum and bladder, showing the percentage of points resulting with $\gamma \leq 1$ 108

Table 7.1 The threshold values for region 1,2 and 3 ...........................................113

Table 7.2: Summary of the prostate treatment plan settings for IMRT .....................114

Table 7.3: NTCP calculation parameters

Table 7.4: Dose and coverage differences between CBCT/sCBCT plans and the pCT plan, in \%, for the PTV, rectum, bladder, left and right femoral head.

Table 7.5: PTV coverage for the pCT and sCBCT plans. The dose to $95 \%$ of PTV volume, D95\%, and the volume receiving $95 \%$ of prescription dose

Table 7.6: Summary of the index with fixed DTA $=3 \mathrm{~mm}$ and DD $=3 \%$ for the calculation points falling inside the PTV, rectum, bladder, right and left femoral head

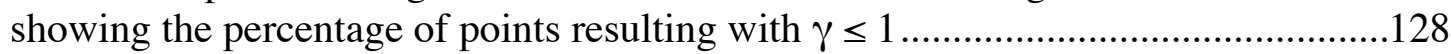

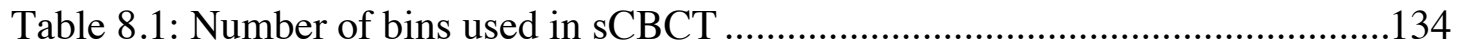

Table 8.2: Summary of the prostate treatment plan settings for IMRT .....................136

Table 8.3: Dose and coverage differences between sCBCT plans and pCT plan in \% for the PTV, rectum and bladder using PB, CC and MC algorithms ........................143

Table 9.1 Summary of the treatment plan settings for IMRT …...............................150

Table 9.2: Dose and coverage differences between the sCBCT plans and the pCT plan, in \%, for the PTV, rectum and bladder using PB, CC and MC algorithms ......154

Table 10.1: Dose and coverage differences between sCBCT plans and pCT plan, in $\%$, for the PTV, rectum and bladder for the standard prostate case 170

Table 10.2: Dose and coverage differences between sCBCT plans and pCT plan, in $\%$, for the PTV, rectum and bladder for the single hip prosthesis case 170

Table 10.3: Dose and coverage differences between sCBCT plans and pCT plan, in $\%$, for the PTV, rectum and bladder for the double hip prostheses case .171 
Table 10.4: Summary of the $\gamma$ index with fixed DTA $=3 \mathrm{~mm}$ and DD $=3 \%$ for the calculation points falling inside the PTV, rectum and bladder, showing the fraction of points resulting in $\gamma<1$ using $\mathrm{PB}, \mathrm{CC}$ and $\mathrm{MC}$ algorithms.

Table 10.5: Conformity index (CI) comparison between pCT, sCBCTman and sCBCTauto plans using PB, CC and MC algorithms 175

Table 11.1: Dose and coverage differences between pCT and sMR plans, in \%, for the PTV, rectum, bladder, left and right femoral head .189

Table 11.2: Summary of the $\gamma$ index with fixed DTA $=3 \mathrm{~mm}$ and DD $=3 \%$ for the calculation points falling inside the PTV, rectum, bladder, right and left femoral head showing the percentage of points resulting with $\gamma \leq 1$ 190

Table 11.3:Dose comparison between pCT and sMR plans at the isocentre using PB, $\mathrm{CC}$ and $\mathrm{MC}$ algorithms 


\section{List of Abbreviations}

\begin{tabular}{|c|c|}
\hline $\mathrm{a}-\mathrm{Si}$ & Amorphous-Silicon \\
\hline ART & Adaptive Radiotherapy \\
\hline CBCT & Cone Beam Computed Tomography \\
\hline $\mathrm{CC}$ & Collapsed Cone \\
\hline CERR & Computational Environment for Radiotherapy Research \\
\hline CFRT & Conformal Radiotherapy \\
\hline $\mathrm{CH}$ & Condensed History \\
\hline $\mathrm{CI}$ & Conformity Index \\
\hline CNR & Contrast-to-Noise Ratio \\
\hline $\mathrm{CPE}$ & Charged Particle Equilibrium \\
\hline CPU & Central Processing Unit \\
\hline CSDA & Continuous Slowing Down Approximation \\
\hline CsI & Cesium Iodide \\
\hline $\mathrm{CT}$ & Computed Tomography \\
\hline CTV & Clinical Target Volume \\
\hline $\mathrm{DD}$ & Dose Difference \\
\hline DCR & Digitally Composited Radiographs \\
\hline DFT & Discrete Fourier Transform \\
\hline DIR & Deformable Image Registration \\
\hline DICOM & Digital Imaging and Communication in Medicine \\
\hline DQE & Detective Quantum Efficiency \\
\hline DRR & Digitally Reconstructed Radiographs \\
\hline DVH & Dose Volume Histogram \\
\hline EBRT & External Beam Radiotherapy \\
\hline ED & Electron Density \\
\hline EPID & Electronic Portal Imaging Device \\
\hline EPL & Effective Path Length \\
\hline ETAR & Equivalent Tissue-to-Air Ratio \\
\hline
\end{tabular}




\begin{tabular}{|c|c|}
\hline EUD & Equivalent Uniform Dose \\
\hline FBP & Filtered Back Projection \\
\hline FDK & Feldkamp, Davis and Kress \\
\hline FIF & Field In Field \\
\hline FOV & Field Of View \\
\hline FPD & Flat Panel Detector \\
\hline GTV & Gross Tumour Volume \\
\hline HU & Hounsfield Unit \\
\hline IGRT & Image Guided Radiotherapy \\
\hline IMRT & Intensity Modulated Radiotherapy \\
\hline IVH & Image Volume Histogram \\
\hline LFOV & Large Field Of View \\
\hline LKB & Lyman-Kutcher-Burman \\
\hline $\mathrm{MC}$ & Monte Carlo \\
\hline MFOV & Medium Field Of View \\
\hline MLC & Multileaf Collimator \\
\hline MLT & Multi-Level Threshold \\
\hline MRI & Magnetic Resonance Imaging \\
\hline MU & Monitor Unit \\
\hline NTCP & Normal Tissue Complication Probability \\
\hline OAR & Organ At Risk \\
\hline OBI & On Board Imager \\
\hline OMP & Oncentra Masterplan \\
\hline $\mathrm{PB}$ & Pencil Beam \\
\hline $\mathrm{pCT}$ & Planning-CT \\
\hline PDD & Percentage Depth Dose \\
\hline PET & Positron Emission Tomography \\
\hline PHSP & Phase Space \\
\hline PSA & Prostate-Specific Antigen \\
\hline PTV & Planning Tumour Volume \\
\hline
\end{tabular}




\begin{tabular}{|l|l|}
\hline QI & Quality Index \\
\hline ROI & Region Of Interest \\
\hline SCD & Source-to-Chamber Distance \\
\hline sCBCT & Segmented CBCT \\
\hline SFOV & Small Field Of View \\
\hline SPR & Scatter-to-Primary Ratio \\
\hline SSD & Source-to-Surface Distance \\
\hline TAR & Tissue-to-Air Ratio \\
\hline TCP & Tumour Control Probability \\
\hline TERMA & Total Energy Released per Unit Mass \\
\hline TFT & Thin Film Transistor \\
\hline TPS & Treatment Planning System \\
\hline TPR & Tissue-to-Phantom Ratio \\
\hline T1 & Longitudinal relaxation time \\
\hline T2 & Transverse relaxation time \\
\hline VRT & Variance Reduction Technique \\
\hline WAB & Water-Air-Bone \\
\hline XVI & X-ray Volumetric Imaging \\
\hline
\end{tabular}




\section{List of publications and academic conferences}

\section{Full papers}

1. Almatani, T., Hugtenburg, R. P., Lewis, R., Barley, S. \& EDWARDS, M. 2016a. Simplified material assignment for cone beam computed tomographybased dose calculations of prostate radiotherapy with hip prostheses. Journal of Radiotherapy in Practice, 15, 170-180.

2. Almatani, T., Hugtenburg, R. P., Lewis, R. D., Barley, S. E. \& Edwards, M. A. 2016b. Automated algorithm for CBCT-based dose calculations of prostate radiotherapy with bilateral hip prostheses. The British Journal of Radiology, 89, 20160443.

3. Almatani, T., Hugtenburg, R. P., Lewis, R. D., Barley, S. E. \& Edwards, M. A. 2017. Dosimetric feasibility of magnetic resonance (MR)-based dose calculation of prostate radiotherapy using multilevel threshold algorithm. Journal of Radiotherapy in Practice, 16, 415-422.

\section{Presentations and posters}

1. Oral presentation at Monte Carlo Neutron Electron and Gamma Radiation Transport Codes meeting (MCNEG, May 2014, Liverpool, UK): Adaptive treatment planning in prostate radiotherapy.

2. Almatani, T., Hugtenburg, R.P., Lewis, R. and Barley, S., Medical Physics and Clinical Engineering in Wales Summer Meeting (June 2014, Cardiff, UK). Poster: Adaptive treatment planning in prostate radiotherapy.

3. Almatani, T., Hugtenburg, R.P., Lewis, R. and Barley, S., 2015 (Electronic poster). EP-1428: Adaptive treatment planning in prostate radiotherapy. Radiotherapy and Oncology, (115), p.S772.

4. Oral presentation at Monte Carlo Neutron Electron and Gamma Radiation Transport Codes meeting (MCNEG, December 2016, Manchester, UK): CBCT- and MR-based dose calculation for prostate radiotherapy using MC modelling. 
Chapter 1 Introduction 


\subsection{Introduction and background}

Prostate cancer is the most common form of cancer in men. In England and Wales about 28,870 men were affected by prostate cancer in 2003, and $31.76 \%$ of them died in 2003 (Cancer Research UK, 2003, Cancer Research UK, 2004). In 2014, statistics published by Cancer Research UK showed that about 46,700 men were diagnosed with prostate cancer, and there were 11,300 prostate cancer deaths in 2014, showing that the number of prostate patients has increased. In England and Wales, 84\% of prostate cancer patients survived for ten years or more. The improvement in survival rates may be due to the prostate-specific antigen (PSA) testing (Cancer Research UK, 2014). It also showed that much effort has been made on improving treatments. There are many types of cancer treatments and these can be used individually or in combination to achieve greater effects. These treatments include: surgery to remove tumours, using drugs to kill or inhibit proliferation of cancer cells by interfering with the process of cell division (chemotherapy), using hormones to stop testosterone from feeding the prostate cancer (hormone therapy), and the use of ionizing radiation to kill or prevent cancer recurrence by damaging the DNA inside the cell (radiotherapy) (Kufe et al., 2003). This thesis will concentrate on treating prostate cancer with external beam radiotherapy (EBRT). In this chapter, a brief introduction and background of radiotherapy process, image guided radiotherapy, cone beam CT and adaptive radiotherapy will be presented. Literature review and previous works of cone beam CT-based dose calculation techniques will be presented as well as thesis aims.

\subsubsection{Radiotherapy process}

The aim of external beam radiotherapy treatment is to maximize the radiation dose to the tumour, whilst minimizing the radiation dose to the organs at risk (OARs) and normal surrounding tissues. The variation in effect with dose on the tumour is called the tumour control probability (TCP) and the variation in effect with dose on normal tissue is called the normal tissue complication probability (NTCP). A high ratio of TCP to NTCP is desired, and this is called the therapeutic ratio (Barrett et al., 2009).

A typical radiotherapy treatment includes a number of different phases that are all taken successively in order to obtain an optimal process and improvements in the therapeutic ratio (Khan and Stathakis, 2010). The first phase of radiotherapy treatment 


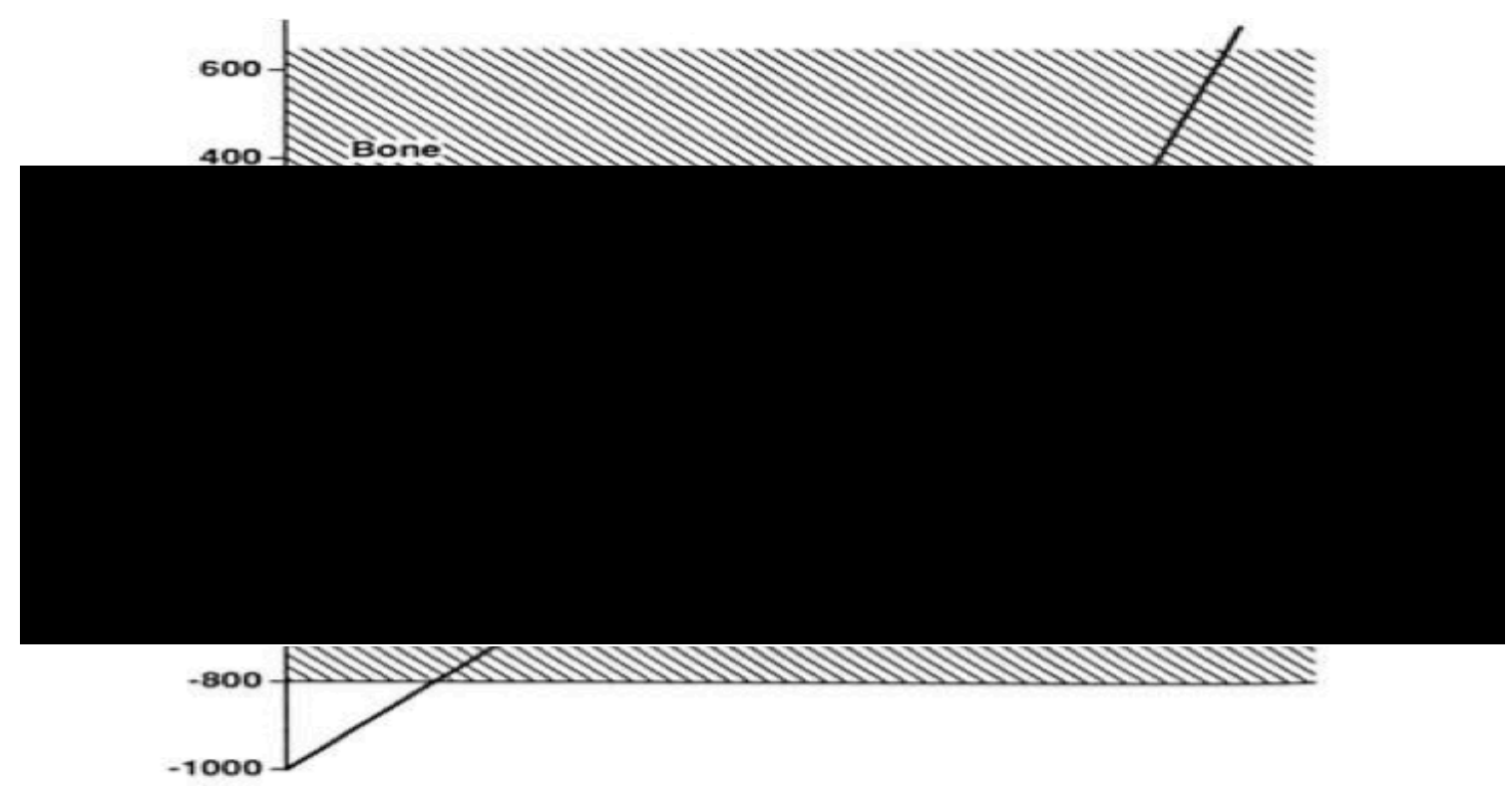

Figure 1.1: Typical calibration curve between CT number and electron density (Khan and Stathakis, 2010).

involves the localization of the anatomical tumour site and surrounding normal tissues which can be assessed by various imaging modalities, such as Computed Tomography (CT), Magnetic Resonance Imaging (MRI) and Positron Emission Tomography (PET). Compared with the conventional radiographs, CT scanners have improved the radiation therapy planning process due to their ability to present a patient's anatomical structure in slices of tissue, without overlying or underlying structures, along with electron density (ED) information that can be used for dose deposition calculations. Thus, in turn, it provides more accurate information.

At Singleton Hospital where this project was undertaken, all radical radiotherapy treatments are planned using CT imaging. CT images are a matrix of relative attenuation coefficients ( $\mu$ ) (Khan and Stathakis, 2010). When reconstructing the CT image, the linear attenuation coefficient is calculated for each pixel, averaged over all energies in the beam spectrum travelling through that pixel from different projections. The reconstructed image does not present these $\mu$ values directly. After CT reconstruction, a high-precision number represents each pixel in the image and this is related to an attenuation coefficient of that pixel. This number is called the CT number and is measured in Hounsfield units (HU).

Compton scattering is the dominant tissue interaction process for megavoltage beams, and it is directly proportional to electron density (Figure 1.1), therefore CT 
provides detailed density information for dose calculations of tissue inhomogeneity e.g. lung and bone, as well as unique anatomical and tumour data (Schneider et al., 1996). Unlike CT, MRI provides excellent soft tissue contrast, which is useful for non-bony sites such as brain, head \& neck and prostate cancers, and do not provide direct electron density information for dose calculation. MRI is the standard imaging for delineating all prostate patients in Singleton Hospital. However, whilst MRI and CT detect any changes that occurred in organs or tissue structures, PET scanning detects the functional variations at the physiological level in early stage before changes in the anatomical structure are visible, and can be used for dose painting (Bentzen, 2005).

Following these imaging procedures the simulation of the treatment course is made, which must be done prior to the treatment planning stage. One of the purposes of simulation in the radiotherapy process is to display the treatment fields so that the target volume can be accurately covered without delivering unnecessary radiation to surrounding normal tissues. In addition, during the treatment course, a simulator can be used to monitor changes in the patients' anatomy arising from their weight loss or tumour progressions/regressions (Aird and Conway, 2002). In modern radiotherapy, a CT scanner and virtual simulation software are used to provide 3D visualization as well as the electron densities required for dose calculation.

The next step is the treatment planning process where the desired dose to the tumour region is maximized to the prescription and is calculated as accurately as possible whilst minimising the dose to the surrounding tissues. At Singleton hospital, patients are asked to follow drinking and dietary protocols to achieve a consistent bladder and rectal volume (see Section 2.3). Beam and patient data acquisitions, the specification of doses and the fractionation steps are part of a typical treatment planning process, where the data is then transferred to the treatment machine and the record-and-verify system (Khan and Stathakis, 2010). A tumour volume is divided into several volumes when prescribing a desired dose to a particular region in a patient. In radiotherapy planning, three of these volumes are of greater importance, as shown in Figure 1.2.

The first volume denotes the demonstrated tumour position and its extent (that can be seen or imaged) and is termed the gross tumour volume (GTV). The second volume comprises the GTV(s) and volumes with suspected tumour that cannot be 


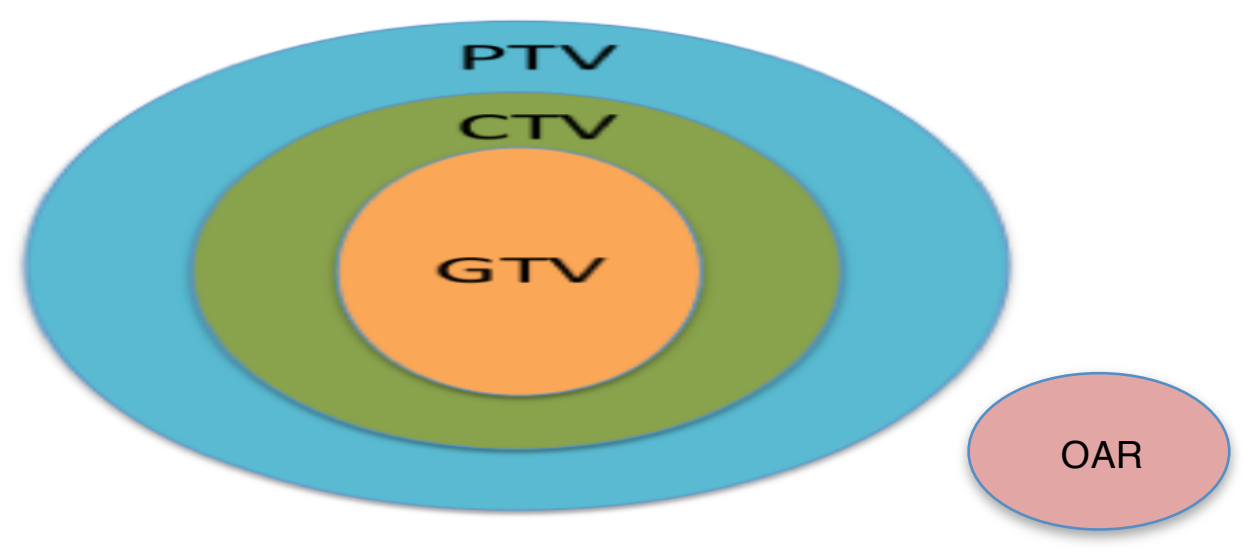

Figure 1.2: A tumour with different volumes

imaged, called the clinical target volume (CTV). Planning target volume (PTV) comprises the CTV with a margin to account for variation in size, shape and position relative to the treatment beams. The PTV is therefore a geometrical concept used to ensure that the CTV receives the prescribed dose (Morgan-Fletcher, 2001). The margin added to CTV to get the PTV is not always isotropic. In this thesis, the CTV was increased by a $1 \mathrm{~cm}$ anterior/left/right/superior/inferior margin and $0.5 \mathrm{~cm}$ posterior margin to give the PTV.

In 3D treatment planning, the selection of an optimum treatment plan can be assisted by a quantitative plan evaluation tool called the dose volume histogram (DVH) (Chen, 1988). The DVH is a graph that shows the calculated dose received by a volume of tissue. DVHs are used to confirm that the desired dose will be delivered to the tumour, whilst the normal structures receive a dose which is within their dose constraints.

As mentioned before, the primary aim of radiotherapy is to maximize the dose to the tumour whilst minimizing the dose to the organs at risk. This is known as conformal radiotherapy (CFRT) where the geometrical shape of a beam can be changed with regard to the tumour site using wedges (Glatstein, 2002). The fluence shaping across the field is constant. Intensity modulated radiotherapy (IMRT) is a particular form of CFRT where both shaping, geometry and fluence, can be adjusted by using many independent leaves in the treatment head, called multileaf collimator (MLC). There are two techniques; the multiple-static MLC (referred to as 'step-and-shoot) and the dynamic MLC technique. IMRT uses an inverse planning technique where the dose 
objectives are set and the treatment planning system (TPS) calculation is optimized to achieve these objectives by making use of DVHs (Glatstein, 2002). IMRT can be used for complex sites and can increase confidence in boosting the dose to the tumour, whilst spreading a modest dose to the surrounding normal tissues.

The treatment planning system used at Singleton Hospital is called the Oncentra Masterplan Treatment Planning System (OMP, version 4.3), and it supports two types of algorithm to calculate the dose distribution: the pencil beam (PB) and the collapsed cone (CC) algorithms (Ahnesjö, 1989, Ahnesjö et al., 1992, Ahnesjö et al., 2005). These model-based algorithms derive the dose distribution using the energy deposition within the patient and the physical characteristics of the treatment beam. In the pencil beam algorithm, an equivalent path length (EPL) correction is used to account for tissue heterogeneities for the primary dose contribution, whilst a onedimensional density correction is used for scattered radiation (see Section 3.4). Although PB provides a fast dose calculation, its accuracy is limited in low-density regions. This is because the PB algorithm does not account for secondary electron transport in media other than water (Knöös et al., 2006). This limitation of the PB algorithm can be overcome by using the $\mathrm{CC}$ algorithm. CC uses three-dimensional density correction to take into account secondary electron transport and energy deposition from scattered radiation (Ahnesjö, 1989). Therefore, CC provides a more accurate dose distribution, particularly in low-density regions, such as interfaces between lung and soft tissues (Knöös et al., 2006).

A more accurate method of dose calculation is the Monte Carlo (MC) algorithm, which is considered to be the gold standard to benchmark dose calculation algorithms (Seco and Verhaegen, 2013). In MC, the primary and secondary electron transport and energy deposition from scattered radiation are taken into account by modeling microscopic interactions (see Chapter 3). In a high dose gradient (i.e. IMRT) or inhomogeneous regions (i.e. lung), the accuracy of MC calculations can achieve $2 \%$ in dose calculation compared with conventional treatment planning algorithms where the accuracy varies between 3-12\% (Francescon et al., 2003, Oelkfe and Scholz, 2006). In this thesis, EGSnrc MC code is used to evaluate the accuracy of PB and CC algorithms (see Chapter 4 and 5).

The critical step in the radiotherapy process is ensuring that the tumour volume 
receives the prescribed dose and the normal tissue structures receive the specified dose within particular clinical constraints as planned. For prostate patients, for example with a prescribed dose of $74 \mathrm{~Gy}$, dose constraints allow no more than $65 \%$ and $30 \%$ of the rectum and bladder be irradiated to $41 \%$ and $68 \%$ of the prescription dose, respectively. Therefore, to assess the accuracy of radiotherapy, geometric and dosimetric verification are needed. Assuming that the patient's external anatomy is constant relative to internal anatomy, tattoos on the skin or bony anatomy are used to align the planned geometry of the field to internal anatomy. For brain cancer, the bony anatomy is a reliable and accurate surrogate for the tumour position. However, both skin and bony anatomies are not accurate surrogates in some tumours, where the movements are independent, e.g. prostate and lung cancers. In these cases, implanting a fiducial marker in or near the tumour as a surrogate for tumour positioning would be important to achieve optimum treatment accuracy. This technique can be used with on-treatment imaging, which is a method that can be used to assess patient positioning prior to irradiation using either 2D or 3D imaging. However, imaging guidance or verification can be divided into online and offline strategies (Hoskin, 2008). An online strategy acquires and assesses information from daily imaging and corrections must be applied immediately prior to each treatment fraction being delivered. An offline strategy refers to frequent acquisition of images taken to match with a reference image at some time after the treatment has been given without immediate intervention. The offline strategy reduces systematic error (i.e. changes in target position and shape between delineation and treatment) whilst the online strategy reduces both systematic and random errors (i.e. changes in target position and shape between fractions, patient set-up errors) (Hoskin, 2008).

\subsubsection{Image guided radiotherapy}

Image guided radiotherapy (IGRT) is used to ensure the radiotherapy process is accurately guided and is achieved by comparing reference images that are produced by the treatment planning system (i.e. digitally reconstructed radiographs (DRRs), digitally composited radiographs (DCRs)) with the patient image in the treatment position acquired by either planar (2D) imaging or volumetric imaging (3D and 4D).

For prostate cancer, bladder emptying and rectal filling have been demonstrated to have significant effects on prostate position and a negative impact on the accuracy of 
the treatment course (Langen and Jones, 2001). At Singleton Hospital, patients are asked to follow drinking and dietary protocols from 4 weeks before the start of treatment (see Section 2.4).

However, these changes usually occur during the course of treatment and repositioning the patient cannot resolve all of them. IGRT, where decisions made on the basis of imaging before and/or during the treatment delivery process, including real-time re-planning, would be one of the ideal solutions leading to improvements in the therapeutic ratio. For patient positioning, many IGRT technologies have been introduced to improve its accuracy such as thermoplastic cast, vacuum bags and portal films (Song et al., 1996, Green et al., 2002, Italia et al., 1997). Later, an electronic portal imaging device (EPID) was introduced which provides a verification image that is obtained by the same MV beam used for therapy (Figure 1.3) (Antonuk et al., 1996). These images can be then compared with the DRRs produced by the treatment planning system. There are some disadvantages with this technique, such as the twodimensional nature of the projection images and the fact that the obtained (2D) image has poor soft tissue contrast due to the MV beam. This technique also imparts an additional MV dose (Shaw, 2014). This suggests that $\mathrm{kV}$ portal imaging should be introduced to reduce patient dose, thus acquiring more projections (Pisani et al., 2000). However, such technologies do not provide soft tissue information. To overcome this limitation, newer linear accelerators have been integrated with $\mathrm{kV}$ cone beam CT (CBCT) systems to produce a set of 3D images with good soft tissue contrast, which can reduce both systematic and random errors (Jaffray and Siewerdsen, 2000, Jaffray et al., 2002). Since its commercial introduction in 2005, the CBCT system has been the main technology for image guidance (Shaw, 2014).

\subsubsection{Adaptive radiotherapy}

The fast development of IGRT technology and more advanced treatment delivery, where a highly conformal dose is used such as IMRT, has produced a need for adaptive radiotherapy (ART) where the initial plan is adapted based on the current patient geometry. ART can address and take into account random and progressive changes, called online and offline ART respectively (Li et al., 2010). For prostate cancer, for example, the online ART approach has been effective at improving daily 


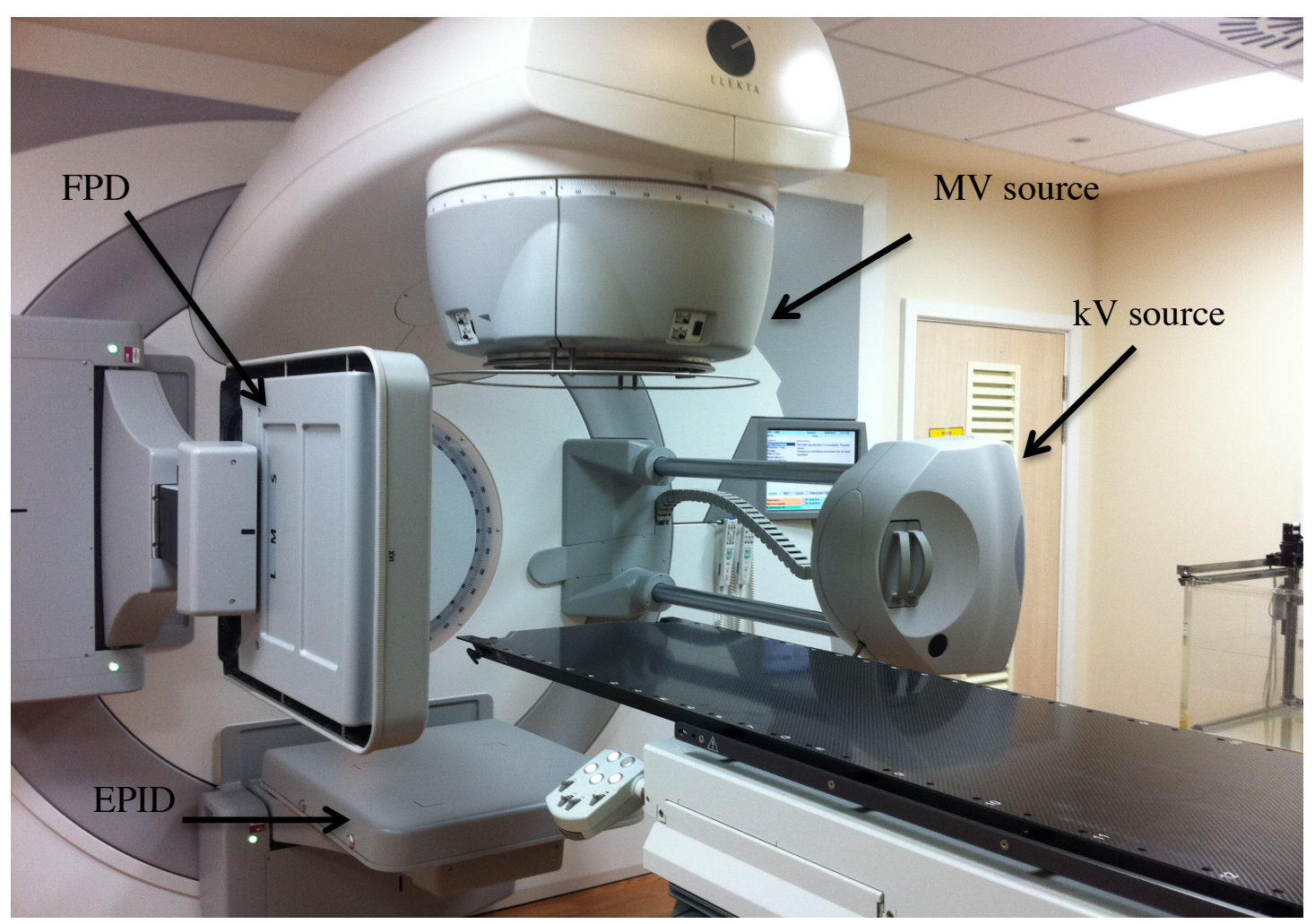

Figure 1.3: Elekta Synergy linear accelerator at Singleton Hospital.

target coverage and OAR sparing by taking into account interfractional/intrafractional motions of the patient. A study showed that using a specified adaptive protocol (prostate localization on CBCT and a diet and mild laxatives) can reduce the PTV by $29 \%$ and the probability for late rectal bleeding by $19 \%$ (Nijkamp et al., 2008).

One of the ART techniques is called 'Plan of the day', where multiple plans are generated at the time of planning with different PTVs. During the treatment course, daily imaging (i.e. CBCT) is used to choose the most appropriate plan based on that day (Murthy et al., 2011, Chai et al., 2012). However, one of the major challenges for online adaptive treatment is the length of time required to re-plan the treatment, especially with IMRT where many different fields among different structures and organs are used depending on the complexity of the tumour site. Considering reoptimization time for the prostate, there are many studies which have proposed different schemes to achieve a fast re-optimization for ART (Wu et al., 2008). However, CBCT represents a technology platform for ART, where the 3D information in $\mathrm{CBCT}$ can be used in many ways such as refining and adapting the planning and target margins for boosting the dose during the treatment course, choosing a "plan of the day", estimating the dosimetric effect of patient weight 
loss/gain, recalculating dose distribution and generating new plans (Smitsmans et al., 2005, Hansen et al., 2006, Nuver et al., 2007, Nijkamp et al., 2008, Chai et al., 2012, Fotina et al., 2012).

\subsubsection{Cone beam computed tomography}

CBCT is a relatively new medical imaging technique that is now available as part of the linear accelerator systems such as Elekta Synergy X-ray volumetric imaging system, which is used in this thesis $\left(\mathrm{XVI}^{\mathrm{TM}}\right.$, version 4.5, Elekta, Crawley, UK) (Figure 1.3 and Figure 1.4). This allows the possibility of imaging the patient in the treatment position to distinguish the exact anatomical information either before or after each treatment in a $3 \mathrm{D}$ view then registering this image to a reference planning $\mathrm{CT}$ image via an integrated software system (Dawson and Jaffray, 2007).

At the beginning, the main aim of introducing CBCT in radiotherapy as an IGRT tool, was to correct patient (translational and rotational) setup errors in the treatment room, as well as to perform quality assurance (see Section 2.2) (Smitsmans et al., 2005, Létourneau et al., 2005b, Moseley et al., 2007, Purdie et al., 2007). The improved CBCT image quality and acquisition time allows this technology to adapt the treatment plan based on daily imaging to match tumour deformation during the treatment fractions and to perform dose calculations (see Section 2.3) (Yoo and Yin, 2006, Boggula et al., 2007, Yang et al., 2007, Dunlop et al., 2015). In order to use CBCT data for dose calculation, it is essential to acquire the relationship between HUs and EDs, as this is the main input for the dose calculation and is used to take into account tissue heterogeneities (Khan and Stathakis, 2010). Compared to a conventional $\mathrm{CT}, \mathrm{CBCT}$ has more scatter due to cone-beam geometry resulting in an increased amount of image artefacts with a lower signal-to-noise ratio produced by the lower photon energy of CBCT (Figure 1.4). This is because the beam is hardened by the preferential attenuation of the lower photon energies leading to less variation between high density and low density structures (Jaffray et al., 2002). In addition, the level of beam hardening and the amount of scatter depends on the field of view, scanned object size, and which collimator and filter are used (see Chapter 2). A CBCT image thus provides inaccurate HUs. Currently, there are two CBCT systems that are widely used in radiotherapy: Elekta XVI and Varian On Board Imager (OBI) (Varian 


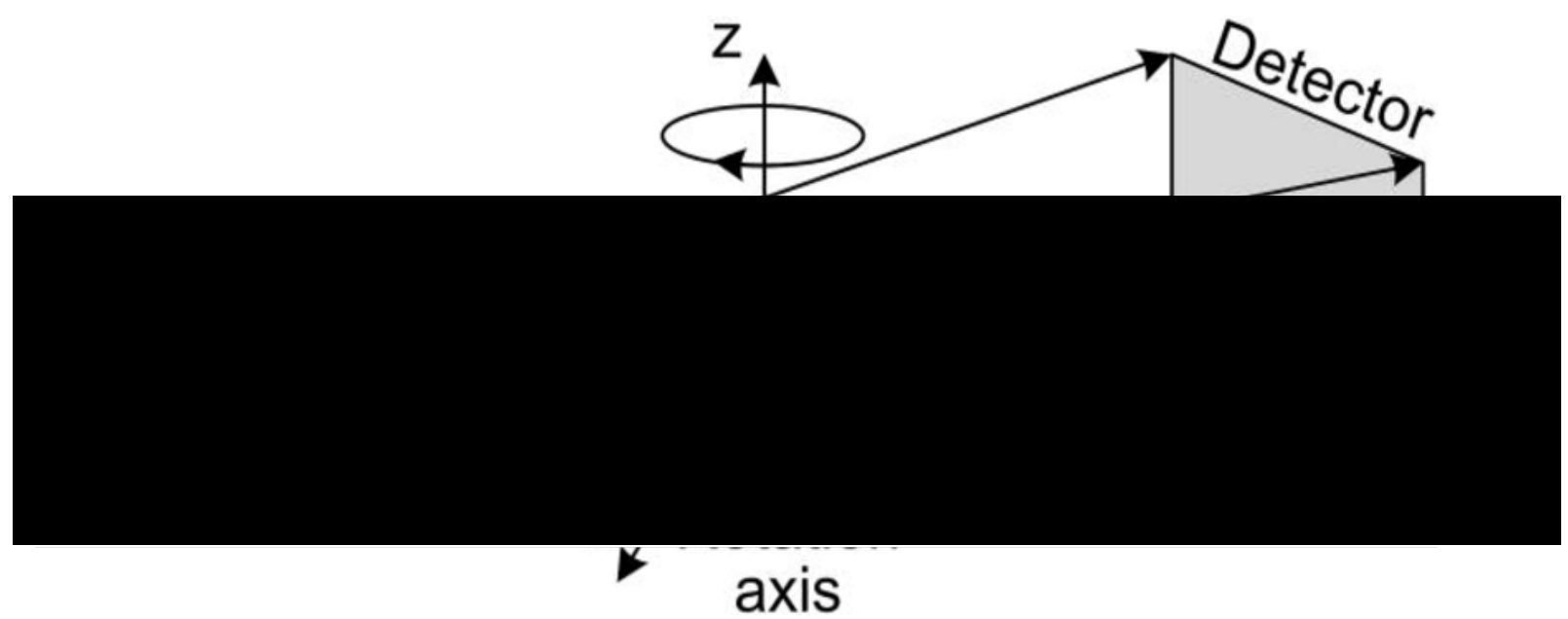

Figure 1.4: Cone beam CT geometry (Liu et al., 2012).

Medical systems, Palo Alto, CA). The Elekta XVI system shows larger discrepancies in CBCT pixel values, and image noise is 2-4 times higher, compared with the Varian OBI system (less than 10 pixel values) (van Zijtveld et al., 2007, Yoo and Yin, 2006, Lee et al., 2008b, Chan et al., 2011). Due to its larger flat-panel detector design, the Elekta XVI CBCT system can cover a larger field of view (FOV). However, the dose to patients from the Elekta XVI is less, and therefore also the image quality, is poorer, than those from the Varian OBI system (Hyer et al., 2010). Therefore, for electron density calibration and dose calculation, the non-uniformity of CBCT images needs to be corrected, as without correction the dose difference between CBCT and CT plans can be up to $51 \%$ in a worst case scenario, and $1-5 \%$ in a best case scenario (Yang et al., 2007, Guan and Dong, 2009, Hatton et al., 2009, Boggula et al., 2009). The main aim of this thesis is to enable dose calculations to be performed with the use of CBCT images taken during radiotherapy course.

\subsection{Literature review and previous work}

The main role of CBCT technology in radiation therapy is to correct patient setup errors as well as to provide tumour or anatomical information such as tumour size, location, internal movement and orientation. As mentioned before, patient weight loss, bladder or rectal volumes and prostate position can change during the course of the treatment, or even between the initial planning CT (pCT) and the first treatment fraction. These changes can be accounted for by acquiring CBCT images, thus 
providing a more conformal target treatment and spare OARs as planned. In terms of its capability in radiotherapy treatment planning, CBCT can provide a more promising future (Shaw, 2014, Rafic and Ravindran, 2015). Ideally, CBCT can be used for dose calculation and to provide information about the treatment dose to the tumour and OARs on a daily or weekly basis for verification and ART purposes.

Owing to its cone-beam geometry, the amount of scatter in CBCT images is greater than that of conventional CT images (fan beam), and is dependent on the scanned object size, the collimator and the filter used. The image quality also depends on acquisition parameters, i.e. $\mathrm{mA}, \mathrm{kV}$ and the number of projections (Hatton et al., 2009). The number of projections for image reconstruction in CBCT is between 400 and 700 which limits the image quality, whilst in pCT it is between 2000 and 4000 projections, thus providing less information about the image (Richter et al., 2008). In addition, limited gantry rotation speed, about 2 minutes, and a large FOV in a single rotation reduce image quality (see Chapter 2). This means that, for each CBCT mode setting and patient site and size, there is a different HU-ED relationship. Currently, CBCT images in the XVI system provide pixel values, gray-scale intensity values or CBCT numbers that do not demonstrate true HUs (Guan and Dong, 2009, La et al., 2009, Li et al., 2013). Consequently, CBCT images cannot be used directly for dose calculation (Fotina et al., 2012). Since the amount of scatter, and consequently CBCT numbers, depend on the patient size and acquisition parameters, converting CBCT numbers into HUs and then establishing a HU-ED calibration curve would cause dose calculation error, in some cases $>5 \%$ (Richter et al., 2008, Guan and Dong, 2009). By comparison, establishing a single HU-ED calibration for a given CT scanner is accurate enough to enable dose calculation in a TPS. Therefore, if there are significant anatomical changes observed on the CBCT images, acquiring another CT is necessary for an accurate assessment of dose differences. This procedure is time consuming across all staff groups involved in the radiotherapy pathway and an additional dose is delivered to the patients. Thus it would be sufficient to use CBCT images that were already taken during radiotherapy for evaluating the necessity of re-planning.

Many papers have studied the use of CBCT data for dose recalculation, which is still an active area of research (Onozato et al., 2013, Li et al., 2013, Onozato et al., 2014, Dunlop et al., 2015, Held et al., 2015, Rafic and Ravindran, 2015). Some of 
these papers look at physical or algorithm modifications to reduce the amount of scatter in CBCT images, whilst some of them deal with the CBCT HU-ED calibration. Other papers used CBCT images for dose calculation by mapping each voxel in $\mathrm{CT}$ to the equivalent point in the $\mathrm{CBCT}$ using rigid or deformable registration algorithms, or by using look-up tables. In addition, approaches to modify CBCT data using density override techniques, such as multilevel-threshold algorithms, can achieve an acceptable dose calculation accuracy. These approaches are relatively unsophisticated and can be easily implemented into clinic.

The first approach, physical modifications, includes anti-scatter grids and a bowtie filter (F1, in Elekta system). It has been shown that using a bowtie filter in Elekta XVI improves image quality and increases the contrast-to-noise ratio (CNR) (Chan et al., 2011). Not only do these modifications reduce scatter artefacts but they also reduce CBCT imaging doses. It has been shown that using a bowtie filter can reduce the dose by $22 \%$ in the pelvic region (Spezi et al., 2012). However, these physical modifications do not always improve image quality to a level where the CBCT images can be used for dose calculation (Marchant et al., 2008, Mail et al., 2009).

Algorithm modifications to reduce scatter can be applied just before processing the projections or by post processing the acquired projections. Rinkel et al (2007) and Morin et al (2007) studied the effect of software corrections to CBCT images on scatter artefacts using both pre- and post-reconstruction, respectively. The result was that a greater reduction in scatter artefacts was observed compared with the physical modifications, but it was not achieved for all object sizes (Rinkel et al., 2007, Morin et al., 2007, Marchant et al., 2008). Poludniowski et al (2012) developed an algorithm to reduce scatter before reconstructing the projections. The results showed that acceptable differences in dose $(<3 \%)$ between the doses on CBCT (Elekta XVI) and pCT can be achieved (Poludniowski et al., 2012). However, such a technique cannot achieve acceptable differences in dose for large patient sizes and can be difficult to be implemented in the clinic. In recent commercial software releases, more sophisticated scatter-reduction algorithms are now available (Dunlop et al., 2015).

The third approach is to generate HU-ED tables for CBCT by assigning the densities in the pCT to the CBCT numbers. Richter at al (2008) studied the accuracy of using CBCT (Elekta XVI) for dose calculation using population-specific HU-ED 
tables. The results showed that the use of phantom based HU-ED tables resulted in dose differences greater than $5 \%$ for pelvis patients. The use of patient specific or patient group specific HU-ED tables resulted in dose differences of less than $5 \%$ (Richter et al., 2008). Hu et al (2010) used patient specific HU-ED tables using a similar method to Richter's. Dose differences of less than 3.3\% were achieved (Hu et al., 2010). However, such techniques are still subject to CBCT image artefacts, which may lead to higher dosimetric errors (Fotina et al., 2012). In addition, in some TPSs, only one intrinsic HU-ED calibration is used and it therefore cannot be routinely altered by the user.

The forth approach for using CBCT images for dose calculation is by modifying the CBCT image where the HU in each voxel in the CT was mapped to the equivalent point in the CBCT. Yang et al (2007) used this approach with a deformable image registration (DIR) for three prostate patients using Varian OBI. The results showed that the dose differences in the PTV could be greater than $6 \sim 10 \%$, which was attributed to the interfractional organ movement. It was concluded that the modifiedCBCT-based dose calculation agreed with the pCT-based dose calculation within $1 \%$ (Yang et al., 2007). Van Zijtveld et al (2007) used the same approach for head and neck cancer patients, but with a rigid registration algorithm based on bony anatomy. The results showed that the dose differences between pCT and CBCT (Elekta XVI) were less than $1 \%$ (van Zijtveld et al., 2007). Depuydt et al (2006) generated a lookup table to convert CBCT numbers into $\mathrm{HU}$ by mapping the $\mathrm{HU}$ values of pCT to CBCT numbers. However, using this approach with DIR, and its associated uncertainties, would in some cases result in no improvement in the accuracy of the dose calculation because the accuracy of DIR can be affected by bowel gas and artefacts from gold fiducial markers inside the prostate (Thor et al., 2011, Onozato et al., 2013). The uncertainty of DIR would be much higher if both pCT and CBCT images were affected by image artefacts, such as metal artefacts caused by the presence of a metal hip prosthesis. In addition, using this approach with rigid image registration would result in higher dose differences as the pCT is usually acquired weeks before the CBCT images (van Zijtveld et al., 2007, Dunlop et al., 2015). Therefore, significant anatomical differences may be observed between the two scans. Furthermore, the method of using look-up tables to convert CBCT numbers into HUs does not take into account the variation in CBCT numbers caused by artefacts in 
different regions of the image (Marchant et al., 2008). Thus significant artefacts present in both histograms would result in higher dose uncertainties.

The last approach for enabling CBCT data to be used for dose calculation is by modifying CBCT numbers using a multilevel threshold (MLT) algorithm. This involves categorising pixel values in the CBCT images into segments of homogeneous HU used in clinical CT. Boggula et al (2007) used the MLT algorithm which replaces the pixel values of CBCT (Elekta XVI) images with three fixed HU values (as in CT) for air, soft tissue and bone based on threshold values. For three prostate cancer patients, the results showed that using uncorrected CBCT images for dose calculation resulted in dose differences of more than $20 \%$ in comparison to the pCT (Boggula et al., 2007). After correcting CBCT images, the dose difference reduced to less than $1 \%$. Onozato et al (2014) evaluated the CBCT-based dose calculation accuracy for 10 prostate cancer patients using two methods: the MLT algorithm and a histogram matching algorithm. Using the MLT algorithm, the CBCT images were segmented into air, fat, muscle and bone with one set of threshold values (Onozato et al., 2014). The study showed that modifying CBCT images using with these two methods with rigid registration resulted in a dose difference of more than $1 \%$ compared with pCT. Using these methods with DIR, the dose differences were reduced to less than $1 \%$. Even though the differences between the two methods, in either registration, were not significant, the histogram matching method provided some correction for CBCT image artefacts caused by the gold fiducial markers and bowel gas, whilst the MLT method erroneously replace it with soft tissue HUs. Such artefacts can affect the DIR accuracy (Thor et al., 2011). The occurrence of additional inhomogeneities in the patient anatomy, e.g. a hip replacement, would significantly affect the DIR accuracy and result in greater dosimetric errors, due to the difficulties presented by the additional metal artefacts in both pCT and CBCT images. In addition, in such circumstances, one set of threshold values would be unable to accurately correct the image artefacts as the level of scatter is variable across the image.

Fotina et al (2012) assessed the accuracy of two HU modifications based on density assignments or overrides, called the "water-air-bone" (WAB) technique and region of interest (ROI) mapping techniques. The ROI technique is based on the same principle 
as the MLT algorithm in a way that the CBCT numbers are replaced by the pCT numbers. In the WAB technique, the bony and low density structures on CBCT images (Elekta XVI) were extracted based on the grey values threshold using an automatic segmentation algorithm (available in iPlan TPS v.4.5.0, BrainLab, Germany) (Fotina et al., 2012). Fixed HU values were then manually assigned for bone (soft bony structures, hard bone and teeth), air/low density regions (rectal balloon and lung) and all the other structures are assumed to be water. The accuracy of these two techniques was compared with the phantom- and population-based HUED CBCT calibration curves approach. For 10 prostate cancer patients, the results showed that the WAB and ROI techniques showed better agreement (below 2\%) with pCT than the two CBCT calibration curves (up to 5\%). Compared with the ROI mapping technique, the $\mathrm{WAB}$ technique is easier to implement in clinic as it does not require special software for mapping or analysis of the average $\mathrm{HU}$ values from the pCT images (van Zijtveld et al., 2007, Fotina et al., 2012).

Recently, Dunlop et al (2015) assessed the CBCT (Elekta XVI) dose calculation accuracy for density override techniques for four pelvic cases, where CBCT voxels were assigned as water-only and then as either water or bone (water-only and waterand-bone methods). This was then compared with a scatter correction with a look-up table and an automated density assignment method that is available in the RayStation TPS (V3.99, RaySearch Laboratories, Stockholm, Sweden) (Dunlop et al., 2015). In the automated density assignment method, six different densities (air, lung, adipose tissue, connective tissue, cartilage/bone, and a higher density for prosthesis) were assigned to the CBCT image by binning the CBCT image histogram into six density levels. Compared with the pCT acquired on the same day as the CBCT, the results showed that the automated approach was superior to the other methods when considering smaller patients (with anterior-posterior distance $<25 \mathrm{~cm}$ ). For larger patients, the water-only method gave the best accuracy. However, the automated density assignment method assigns the six materials based on the CBCT image histogram, which is approximated by two normal distributions to define the threshold values. Therefore, for larger patients, thus larger scatter, this approximation is highly affected, leading to larger dose differences. If there are significant image artefacts, such as metal artefacts, the approximation is even more affected. For the water-only method, all tissues are assigned as water and it ignores lower-density tissues, such as 
adipose, and higher-density tissues, such as bone, thus provides a less accurate anatomical representation.

Besides dose calculation uncertainties introduced by CBCT image artefacts, or by the approaches used to enable dose calculation on CBCT images, dose calculation algorithms can be a source of dosimetric uncertainty. Some of the presented studies used moderately simple dose calculation algorithms, such as the pencil beam algorithm (Yoo and Yin, 2006, van Zijtveld et al., 2007, Yang et al., 2007, Boggula et al., 2009, Hatton et al., 2009, Guan and Dong, 2009). It is known that pencil beam algorithms calculate dose to water (Ahnesjö et al., 1992, Ahnesjö et al., 2005). For patients with additional inhomogeneities, such as hip prostheses, such an algorithm can result in incorrect interpretations. None of the presented papers studied the effects of using different dose calculation algorithms on the processed CBCT images of a patient with additional inhomogeneities.

\subsection{Thesis aims}

As mentioned before, changes in patient position, tumour shrinkage or growth, and internal organ motion occur during the treatment course for prostate cancers. For example, bladder and rectal volumes change on a daily basis, affecting the prostate position. The $\mathrm{CBCT}$ and $\mathrm{pCT}$ images are fused based on rigid registration. After the first three fractions, if the average shift in CBCT and pCT match is more than the tolerance of $3 \mathrm{~mm}$, which takes into account the uncertainties in outlining, registration and position, then it will be corrected for the next fraction. Acquiring CBCT images with the new setup in the next fraction, if it is still more than $3 \mathrm{~mm}$ this means that there are significant changes in patient geometry and/or internal organ position/shape seen on the CBCT scan, then a dosimetric assessment is required. Then the original plan is copied onto CBCT images after creating an external contour and fusing it with the pCT images, as shown in Figure 1.5. The dose is then recalculated turning the inhomogeneity correction off in both $\mathrm{CBCT}$ and $\mathrm{pCT}$. If the dose difference at the isocentre is within $2 \%$, the treatment will be continued, whilst if it is more than $2 \%$, the patient will be re-scanned in the CT and the original plan is copied onto the new $\mathrm{CT}$. The dose is then recalculated turning the inhomogeneity correction on. The Oncologist will review the dose distribution on the new scan and if acceptable, the 


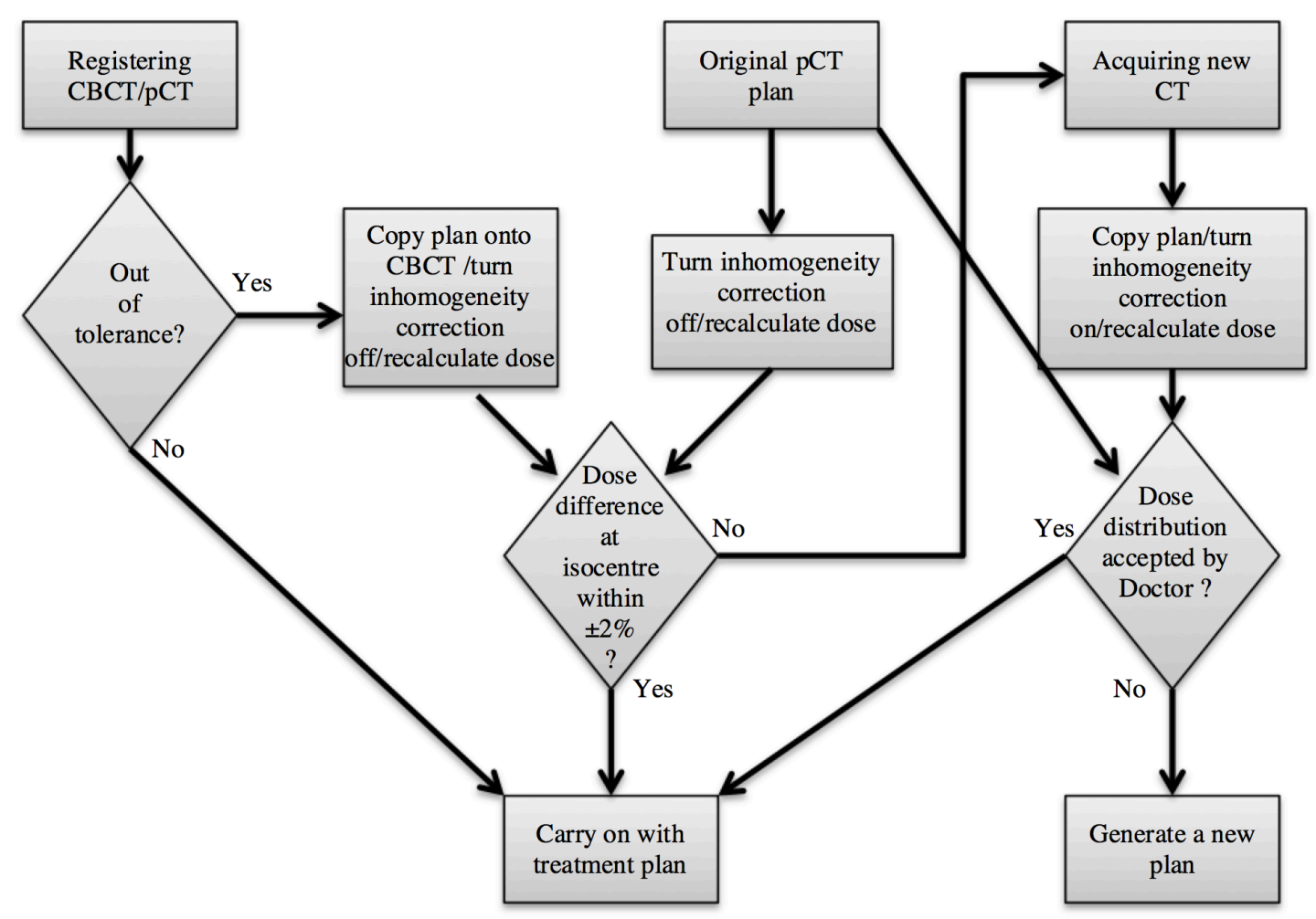

Figure 1.5: Current verification process using CBCT images.

treatment will be continued, otherwise a new treatment plan will be generated.

The main aim of this thesis is to correct/modify CBCT images that are obtained during the treatment course, perform dose calculation on these processed images and to assess the impact of the dose being delivered. Since the CBCT images contain more scatter than a conventional CT system, the CT HU-ED calibration should be changed based on the CBCT image. Despite the fact that the accuracy of using HUED CBCT calibration is affected by image artefacts, this cannot be done with the existing treatment planning system software (OMP, version 4.3), where the CT calibration is intrinsic and cannot be altered by the user. As an alternative, the MLT algorithm will be used to modify/segment CBCT images for dose calculation, as it is more robust against CBCT artefacts and can be easily implemented into clinic. In this thesis, the MLT algorithm will be used to segment CBCT images and will be investigated, along with the following objectives:

1. Investigating the dose calculation accuracy based on segmented CBCT images using MLT algorithm.

2. Segmenting CBCT images of a phantom, patient, patient with a single hip 


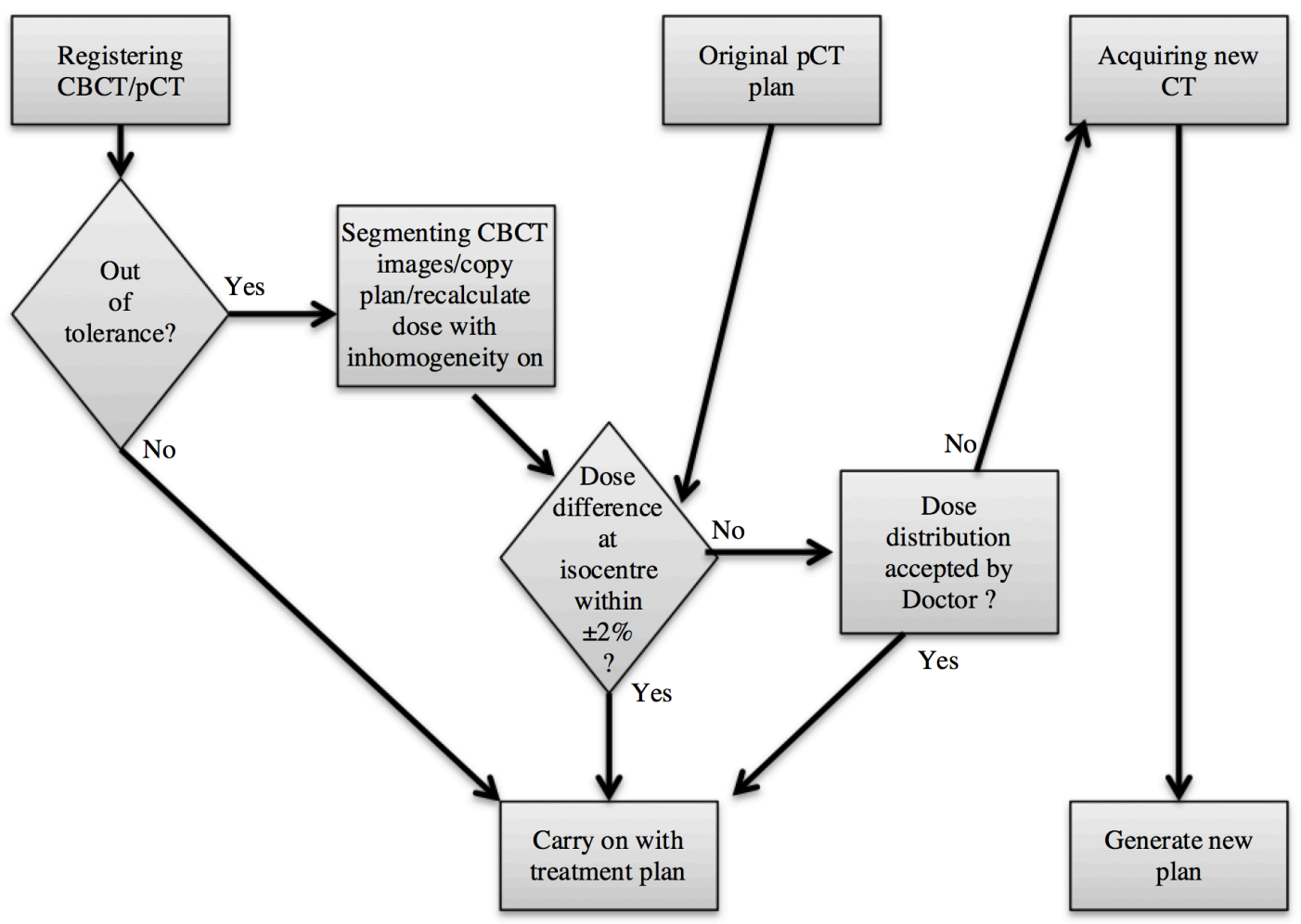

Figure 1.6: Verification process using modified/corrected CBCT images.

prosthesis and patient with double hip prostheses, to provide a good assessment of dose calculation.

3. Finding the optimum number of material bins, where a bin is a region of a uniform $\mathrm{HU}$ value, that are needed when segmenting CBCT images to give the best balance between dose accuracy and operator time.

4. Identifying the effects of these bins on the dose calculation algorithms when using simple and complicated plan geometries.

5. Developing the MLT algorithm to automate the CBCT segmentation process based on geometric landmarks and radial symmetry and enhancing the performance of this method so it can be completed in a timeframe which can make it clinically useful. Then using it as a fast decision-making tool regarding ontreatment patient shape changes and whether a new $\mathrm{CT}$ is required.

6. Enabling dose calculations to be performed with the use of MR images using MLT algorithm.

To identify the effects of the segmentation process on the dose calculation algorithms, Monte Carlo modeling will be used, firstly in a conventional way to test uncertainties in the dose calculation inherent in the treatment planning system, and 
secondly to enable dose calculations to be performed with the use of CBCT images. In this way, it minimizes uncertainty related to the dose calculation as well as identifying those introduced by the different scanning technologies and different material bins.

The endpoint of this thesis is to have an accurate and efficient way to modify CBCT data where it can be quickly used for dose calculation that is as accurate as using the TPS to calculate a dose distribution on a CT planning scan, then using it as a quickdecision-making tool for re-planning, as shown in Figure 1.6. This will eliminate the need to rescan the patient (unnecessary CT rescan if the patient does not require a new plan to be created) and potentially avoiding additional dose and enabling the introduction of adaptive planning in the clinic.

\subsection{Thesis structure}

Having established the research activity in the subject area, the rest of the thesis is structured in the following:

Chapter 2 reviews the physics of cone beam CT systems in detail, including system instructions, applications and image artefacts.

Chapter 3 reviews the advantages and disadvantages of some of the commercial dose calculation algorithms including Monte Carlo dose calculation algorithm.

Chapter 4 presents the main concepts of Monte Carlo simulation for photon and electron transports. It also reviews the main radiation interaction mechanisms.

Chapter 5 shows the investigation and validation of the Monte Carlo linear accelerator model of the Elekta Synergy linear accelerator using EGSnrc MC code.

Chapter 6 investigates the dose calculation accuracy based on modified/segmented cone beam CT images of a male pelvis phantom. The segmentation process was performed using the multilevel threshold algorithm and two material bins were included, water and bone. 
Chapter 7 investigates the dose calculation accuracy based on segmented cone beam CT images of a prostate patient. The segmented cone beam CT images included three bins that represent air, water and bone.

Chapter 8 shows the investigation of the dose calculation accuracy based on segmented cone beam CT images of a prostate patient with hip prosthesis. A large number of material bins (up to 8) was explored to find an optimum solution, balancing, segmentation speed (operator time) with dose calculation accuracy. This chapter and parts of Chapter 6 are coauthored publications, which I laid on the development of the methodology, analysis and the write-up (Almatani et al., 2016a).

Chapter 9 shows the investigation of the dose calculation accuracy based on segmented cone beam CT images of a prostate patient with double hip prostheses. Based on Chapter 8 results, five material bins were used to segment cone beam CT images. The same cone beam CT image set was segmented three times each with different operator time to provide more accurate bone delineation.

Chapter 10 introduces an automated multilevel threshold algorithm to reduce operator time associated with the multilevel threshold algorithm. The automated multilevel threshold algorithm was used to segment the same cases used in Chapter 7, 8 and 9. Parts of this chapter (double hip case only) are coauthored publications, which I laid on the development of the methodology, analysis and the write-up (Almatani et al., 2016b).

Chapter 11 investigates the segmentation of MR images using the multilevel threshold algorithm and the dose calculation accuracy based on segmented MR images of the prostate cancer patient used in Chapter 7.

Chapter 12 summarises the findings of this thesis, provides suggestions for future works and concludes the thesis. 
Chapter 2 Cone beam computed tomography 


\subsection{Introduction}

CBCT is a medical imaging technique that was initially developed as an IGRT tool by Jaffray and Siewerdsen in the early 2000's (Jaffray and Siewerdsen, 2000, Jaffray et al., 2002). Since then it was characterized and implemented in radiotherapy departments as part of the linear accelerator (Linac) system to correct patient (translational and rotational) setup errors in the treatment room in order to reduce field margins and to optimize the treatment plan (Figure 1.3 and Figure 1.4) (Smitsmans et al., 2005). Compared with fan beam (conventional) CT, CBCT uses a beam that is wider in the patient longitudinal direction and it is possible to acquire a wider volume scan in a single rotation, making it feasible to be integrated into Linac systems. By comparison, the fan beam property in the conventional CT, as well as the mechanical complexity of the implantation, means it is practically infeasible to integrate conventional CT with the Linac. Siemens SOMATOM CT-on-rails, installed in the treatment room, has been used but not widely due to its limitations, such as uncertainties in the patient couch position after a rotation. This is because the CT-onrail dose not share the same gantry with the treatment machine, operating independently (Owen et al., 2009). Currently, there are several linear accelerators integrated with $\mathrm{kV}-\mathrm{CBCT}$ systems (sharing the same gantry) which are commercially available. The more commonly used systems are Elekta X-ray Volume Imager (XVI) (Elekta, Crawley, UK) and Varian On Board Imager (OBI) (Varian Medical systems, Palo Alto, CA). This study focuses only on the XVI system (XVI ${ }^{\mathrm{TM}}$, version 4.5 , Elekta, Crawley, UK) mounted on the Elekta Synergy Linac (Figure 1.3).

\subsection{XVI system}

The XVI system contains a kilo-voltage $(\mathrm{kV})$ source, which is similar to those used for conventional $\mathrm{CT}$, and has a fan-cooled X-ray tube and a flat panel detector. The term "cone beam" refers to the cone-shaped beam generated from the $\mathrm{kV}$ source. Kilo-voltage X-rays are generated by an X-ray tube, which is mounted on the linear accelerator drum so that the focal spot of the tube is at $90^{\circ}$ to the mega-voltage (MV) source and $100 \mathrm{~cm}$ from the treatment system axis of rotation as shown in Figure 1.3. The flat panel detector is mounted opposite the X-ray source and the MV treatment beam shares the same axis of rotation with the $\mathrm{kV}$ beam. The central axis of the $\mathrm{kV}$ beam is positioned perpendicular to the treatment beam. Two retractable arms control 
the movement of the $\mathrm{kV}$ source and the flat panel detector, and the source-to-detector distance is $153.6 \mathrm{~cm}$. In conventional CT scanners, the acquisition can be performed using either axial or helical modes. For the former, the scans are performed with multiple rotations at different axial positions whilst in the latter, the scans are performed with multiple rotations with a table moving at a constant speed. By comparison, the acquisition of CBCT images is performed by taking multiple sequential planar projection images of the FOV, acquired in a single rotation around the patient with a fixed table position (axial mode). Each planar image is projected and collected at different angles and for each scanning protocol there are a different number of projections acquired.

The $\mathrm{kV}$ beam is filtered as it exits the tube housing by a physical filtration system, made of a stack of metallic cones and disks, that is fitted at the tube window. This system reduces the dose to the patient and decreases scatter. An additional physical filter, called a bowtie filter, can be added by the user to further reduce the amount of scattered radiation which in some CBCT scans, such as in the pelvic region, exceed the primary radiation (Graham et al., 2007). The bowtie filter (F1) is constructed of aluminium and is contained in a cassette that can be placed in the beam path. With the bowtie shape, the signal at the periphery of the field is reduced as the photons travelling toward the periphery traverse a greater thickness of aluminium, allowing the detector panel to operate within its dynamic range. It improves image quality by removing low energy photons and reducing the amount of scattered reaching the detector by a factor of 2 (Siewerdsen and Jaffray, 2000, Graham et al., 2007).

The flat panel detector can be placed in three different positions (perpendicular to the rotational axis of the imaging system) to generate three different FOVs: small, medium and large. These different fields of view determine the transaxial dimensions of the image (XVI R4.5 manual). The small FOV (SFOV) is selected by centrally aligning the flat panel with the X-ray tube and a minimum gantry rotation of $200^{\circ}$ is required. The maximum diameter of this scan is $27 \mathrm{~cm}$ at the isocentre. For the medium FOV (MFOV), the flat panel is shifted laterally by $11.5 \mathrm{~cm}$ and the reconstructed images can have a diameter of $41 \mathrm{~cm}$. When the flat panel is offset from the central axis of the $\mathrm{kV}$ beam, the circular movement around the axis results in a wider volume being scanned. The large FOV (LFOV) is selected by offsetting the flat 
panel to $19 \mathrm{~cm}$ from the central axis. A full gantry rotation is required for both medium and large FOV.

For each FOV there is a collimator cassette which contains a lead insert with a specified shape and a thickness of $3.2 \mathrm{~mm}$. The collimators are inserted to restrict the $\mathrm{kV}$ beam to the FOV and to determine the scan length in the superior-inferior direction along the patient (G-T direction). This is called the nominal irradiated length at the isocentre by Elekta.

\subsection{1 kV source}

The $\mathrm{kV}$ source generates $\mathrm{X}$-rays by converting the kinetic energy of electrons accelerated under a high voltage into electromagnetic radiation due to 'collisional' or 'radiative' interactions with a metal target (see Chapter 4). The X-ray production is controlled by an X-ray tube and an X-ray generator, which supplies electric power and allows the user to control the selection of X-ray energy. The X-ray tube (Dunlee, Aurora, IL) is a vacuum tube and comprises a cathode and anode. The cathode is a heated tungsten filament from which electrons are emitted, termed thermionic emission. The XVI uses a rotating anode that contains the tungsten target with a small angle, $14^{\circ}$, and a focal spot size of $0.8 \mathrm{~mm}$ (Mail et al., 2009). The emitted electrons are accelerated toward the anode by a high voltage, supplied by the X-ray generator, without collisions since the X-ray tube is a vacuum, to generate the X-ray. The X-ray tube is capable of producing photon spectra with peak voltage $(\mathrm{kVp})$ range between 70 and $150 \mathrm{kVp}$.

\subsubsection{Flat panel detector}

A flat-panel detector (FPD) is an indirect detector that is based on a large-area solidstate sensor panel coupled to an X-ray scintillator layer. The FPD in the XVI system is an amorphous-silicon (a-Si) thin film transistor (TFT) on a large area of scintillating material (cesium iodide (CsI)) detector and has a matrix of $1024 \times 1024 \times$ 16 bits (physical size $41 \times 41 \mathrm{~cm}^{2}$ ). With indirect detection of X-rays, an X-ray tube sends a beam of X-ray photons through a target. X-ray photons not absorbed by the target strike the CsI scintillator layer, where the photoelectron effect occurs. The electrons in the scintillator are then excited and lose their energy through ionisation 


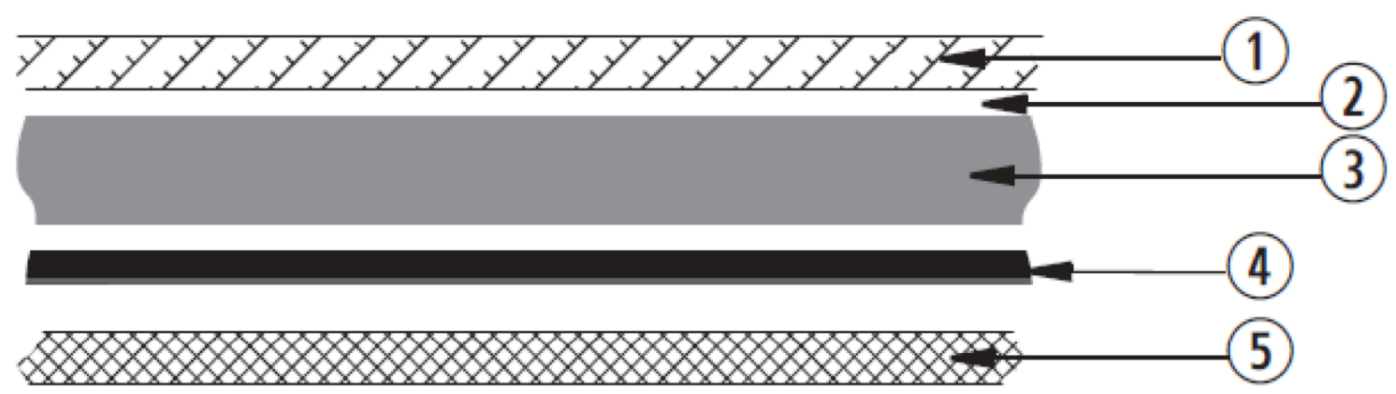

Figure 2.1: Construction of flat panel detector, 1 Aluminum, 2 air gap, 3 CsI scintillator, 4 attenuator and 5 photodiodes (Courtesy of Elekta)(Giles, 2010).

interactions. This creates electron-hole pairs, which are positively charged holes and negatively charged electrons. The electron-hole pairs then recombine producing visible light, which has an intensity that is proportional to the energy of the incident photon. These visible light photons are detected and converted into electrons by the TFT layer of the photodiode that can activate the pixels in a layer of amorphous silicon. The activated pixels produce electronic data that a computer can translate into an image of the target. The FPD produces images with sufficient soft tissue contrast at acceptable dose levels. It can also cover a large field of view with a resolution of approximately $0.5 \mathrm{~mm}$ (Chan et al., 2011). In addition, there are other advantages of the FPD which include large-area fabrication and radiation damage resistance. However, there are some disadvantages that limit their performance, such as manufacturing defects, their non-uniformity and their non-linear response to the radiation spectrum. Furthermore, the material characteristics of the amorphous-silicon semiconductor shows increased charge trapping effect and lower mobility (Bourland, 2012). These limitations of the FPD produce image artefacts and thus decrease image quality, which are discussed in detail in Section 2.5.

\subsubsection{Image reconstruction}

In conventional $\mathrm{CT}$, the algorithm used for image reconstruction is called Filtered Back Projection (FBP), which is the most widely used algorithm in CT image reconstruction (Turbell, 2001). The X-ray runs through multiple slices and each slice is reconstructed to give $3 \mathrm{D}$ volumetric data. $\mathrm{In} \mathrm{CBCT}$, however, the $2 \mathrm{D}$ projection data is used directly to reconstruct $3 \mathrm{D}$ volumetric data. This is referred to as conebeam reconstruction where the projection data is acquired with a circular trajectory. 
The most popular algorithm used for CBCT image reconstruction, and that which is also used by the XVI system, is called the Feldkamp, Davis and Kress (FDK) algorithm, also referred to as the Feldkamp algorithm (Feldkamp et al., 1984, Shaw, 2014). The FDK algorithm is an extension of the fan beam FBP algorithm, where the 2D cone-beam projections undergo three major steps; preweighting, 1D filtration and 3D backprojection. The preweighting process depends on both the fan angle, which defines the width of the beam across the scanned object, and the cone angle, which defines the width of the beam in the object direction. The preweighting process compensates for the increased attenuation of photons along the periphery of the object at larger cone angles. Then a row-by-row ramp filtering is applied, on the detector plane, to the preweighted data to remove statistical noise. During the 3D backprojection step, the intensities in each voxel for a particular cone angle are determined by linearly interpolating all filtered fan-beam projections along the tilted plane and these are then summarized to get the whole 3D volume.

Due to its approximate nature, the structures in planes that are away from the midplane are inaccurately reconstructed. This is due to the fact that the projection data acquired along circular source-detector trajectory are inadequate for complete reconstruction. According to the so-called Tuy-Smith condition, a complete reconstruction can be obtained if every plane intersecting the object intersects the source-detector orbit (Tuy, 1983, Smith, 1985). For CBCT, consequently, any location above or below the midplane violates Tuy-Smith condition. This incompleteness of the circular cone beam acquisition results in the so-called cone beam artefacts that will appear near the edges of the image and these become more prominent with increasing cone angles (see Section 2.5.6). Despite its approximate nature, the FDK-type algorithms are easy to implement and have higher computational efficiency to provide a quick volume image.

\subsection{CBCT in image-guided radiotherapy for prostate cancer}

The main aim of EBRT is to deliver the planned radiation dose to the target volume, PTV, and to avoid delivering high doses to OAR. With the development of highly conformal treatment techniques, such as IMRT, it becomes possible to deliver higher doses to the target, dose escalation, and whilst also minimizing the dose to the 
neighboring normal tissues. For the prostate, such technique results in better clinical outcomes such as increasing local tumour control (Sveistrup et al., 2014). It is been shown that dose escalation of $\geq 75.6 \mathrm{~Gy}$ for low-risk patients were associated with improved long-term prostate-specific antigen relapse-free survival (PSA-RFS) outcomes, and for higher-risk patients, biochemical control was improved with $\geq$ 81 Gy (Zelefsky et al., 2011). Thus greater precision for localization of the target, OAR, treatment set-up and delivery is required.

However, the adjacent organs, such as the rectum and bladder, and their interrelationship, the distension of the rectum and bladder filling makes delivering a uniform dose to the target challenging on a day-by-day basis. The delivery of a typical prostate radiotherapy course takes about 7 weeks and is planned on a single pCT scan that is taken a week or two before starting the course. As mentioned in Chapter 1, it has been shown that rectal and bladder volumes have significant impacts on the prostate position (Roeske et al., 1995, de Crevoisier et al., 2005). The rectal distension has a larger influence than the bladder filling. In addition, tumour regression and patient weight loss might also occur during the treatment course. Thus the initial pCT scan is more unlikely to accurately represent either the prostate position or the surrounding normal tissue. This variation of the internal anatomy increases the risk of geographical miss and decreases the dose to the target with increasing dose to OAR, leading to low biochemical control and local control, and increased rates of especially late rectal toxicity (de Crevoisier et al., 2005, Fiorino et al., 2009).

These changes in prostate position can be up to $11 \mathrm{~mm}$, primarily in the anteriorposterior (AP) direction and $13 \mathrm{~mm}$ superiorly due to rectal size and shape (Ciernik et al., 2002, Hoskin, 2008). Interfraction and intrafraction motions have major impacts on the treatment as well. Interfraction motion is the motion of the anatomy between different treatment fractions and has both systemic and random factors such as variability in target position between fractions whilst intrafraction motion is the motion occurred during a single fraction and considered to be random such as breathing and internal organ motion (Hoskin, 2008). To compensate for these motions, the CTV-PTV margin should be more extended. However, a larger PTV margin increases the dose of the surrounding normal tissue. For prostate volume of 60 $\mathrm{cm}^{3}$, a $55 \%$ or $74 \%$ reduction of normal tissue irradiated is achieved when decreasing 
the margin from $10 \mathrm{~mm}$ to $5 \mathrm{~mm}$ or $3 \mathrm{~mm}$, respectively (Ciernik et al., 2002). BodaHeggemann et al (2011) stated that reducing standard margins by 50\% leads to decreased biochemical control and increased rectal toxicity since the margins are too small to consider interfractional motion. Van Herk (2008) stated that biological imaging so far is not capable of showing tumour deposits on a microscopical level due to the resolution limitation of all scanners and therefore CTV-PTV margin should take that into account to avoid missing part of the tumour.

As a result, it can be clearly seen that the margin is the result of compromises that balanced concerns for a potential geometrical miss, unacceptable toxicity and tumour control, especially when a high dose is delivered. It has been shown that patients with a distended rectum in the $\mathrm{pCT}$ lead to a reduction in biochemical control rates (de Crevoisier et al., 2005). In the UK, approximately $20 \%$ of patients need repeat planning CT to reduce rectal volume (Stillie et al., 2009). Therefore, a dietary protocol to achieve a consistent rectal volume is required to maintain prostate position and improve biochemical control with a decrease in rectal toxicity (Langen and Jones, 2001, Stillie et al., 2009). In addition, it has been shown that it is more advantageous to treat prostate with a full bladder protocol to reduce bladder toxicity (Hille et al., 2005, Hoskin, 2008). In Singleton hospital, the drinking and dietary protocols include laxatives (Liquid paraffin and Magnesium Hydroxide $(30 \mathrm{ml})$ ) to ensure regular bowel movements with the aim of reducing rectal diameter, and drinking water $(>200$ ml) to maintain comfortably full bladder size. However, an advanced imaging guidance technique, IGRT, is thus required to localize the target and evaluate OAR geometry throughout the radiotherapy treatment course.

As a CBCT system is integrated with a Linac, it allows correcting patient set-up and provides $3 \mathrm{D}$ image sets of the patient in the treatment position immediately before treatment with good soft tissue contrast (Jaffray et al., 2002). It can also monitor any changes in shape, size and position of the prostate and those related to weight loss, organ filling/distension or tumour regression. This can be done by registering CBCT images with the pCT images (rigid or deformable registration) that also determine the shift required to place the patient in the correct position as planned. The development of CBCT volumetric imaging in the treatment room makes it a widely-used IGRT tool and a routine procedure for patient verification and tumour positioning, especially for 
highly conformal radiation therapy techniques (Boydev et al., 2015, Held et al., 2015). Along with the prostate, the accuracy of radiotherapy treatment has been improved by using CBCT as an IGRT tool for different sites such as head \& neck and lung cancers (Boda-Heggemann et al., 2011, Oldham et al., 2005).

The use of CBCT as an IGRT tool has been shown to allow a reduction in CTVPTV margins and a reduction in toxicity. It has been demonstrated that CBCT can achieve a high accuracy of matching CBCT images of an unambiguous object to reference pCT images with residual errors $0.12 \mathrm{~mm}$ (Moseley et al., 2007). For prostate phantom, the accuracy of CBCT-based IGRT is high with residual errors less than $1 \mathrm{~mm}$ (Létourneau et al., 2005a). This is more difficult to achieve in clinical practice, where inter-observer variability can be $2.2 \mathrm{~mm}$ when matching CBCT images of soft tissues to a reference pCT images (Moseley et al., 2007). This can be reduced to less than $1 \mathrm{~mm}$ if fiducial marker was used for prostate localization (BodaHeggemann et al., 2011). Deegan et al (2015) studied the difference between aligning fiducial markers and soft tissue on $\mathrm{CBCT}$ and found that the inter-observer variability on soft tissue-based alignment was higher than fiducial marker-based alignment, which should be considered in the margin generation. For soft tissue-based alignment, the higher inter-observer variability can be due to CBCT image quality, which can be affected by different image artefacts (see Section 2.5, for more details).

Even though the CBCT image quality is inferior to the pCT, CBCT has the advantage over 2D-based IGRT to evaluate OAR geometry. A study showed that the rectal and bladder borders were reliably identified in $94 \%$ of the CBCT image sets of 176 cases (Showalter et al., 2008). The cases were without any addition of inhomogeneities into the patient anatomy such as hip replacement which greatly degrades image quality. A proper bladder filling protocol allows the reduction of the dose to OAR and the PTV margin from $8 \mathrm{~mm}$ to $4 \mathrm{~mm}$ (Pawlowski et al., 2010). Further using CBCT with margin reduction to $3 \mathrm{~mm}$ statistically improves rectal toxicity as modeled by NTCP with no reduction in the TCP (Maund et al., 2014).

Beside image quality, the use of CBCT as an IGRT increases overall radiation dose to the patients. It has been shown that $\mathrm{kV} \mathrm{CBCT}$ of the pelvis resulted in an effective dose of more than $22 \mathrm{mSv}$ per scan and could increase the secondary cancer risk by up to $2 \%$ to $4 \%$ if CBCT is used daily (Kan et al., 2008). Mandatory in UK 
legislation, CBCT has to be used safely in IGRT and therefore, the risks associated with the concomitant imaging need to be balanced against the benefits of the treatment outcome such an increase in overall survival, improvement in TCP and reduced toxicity (Sykes et al., 2013).

\subsection{CBCT in adaptive radiotherapy for prostate cancer}

The initial pCT that is acquired one or two weeks before starting the treatment course is unlikely to accurately represent the prostate position or the surrounding normal tissue due to changes in the patient anatomy. Changes such as tumour shrinkage, weight loss and OAR deformation cannot be corrected accurately unless the treatment is re-planned or adapted to take these changes into account. Adaptive radiotherapy (ART) uses CBCT images as a guidance to facilitate re-planning, then adapting or modifying the treatment prescription parameters, such as number of fraction or even generating a new plan, based on any changes in the patient anatomy prior to the treatment. Another treatment prescription parameter that can be modified is field margins (as mentioned in Section 2.3). The first clinical results of CBCT as ART evaluated for prostate cancer were found to reduce the PTV margin by $29 \%$ on average and reducing the probability of late rectal bleeding by $19 \%$ (Nijkamp et al., 2008, Srinivasan, 2015). ART combined with IGRT can also compare the planned and delivered doses to the target volume and OAR.

The use of CBCT as an ART tool can be used to generate treatment plan, estimate the dosimetric effect of patient weight loss, assess choosing a 'plan of the day' and recalculate the dose distribution (Wu et al., 2008, Cheung et al., 2009, Burridge et al., 2006, Boggula et al., 2007). To estimate the dosimetric impact of observed anatomical changes, the original plan is applied to the CBCT images and the dose distribution within specific volumes is compared with the original dose distribution. In the socalled 'plan of the day', multiple plans are generated at the time of planning with different PTVs. During the treatment course, daily CBCT imaging is used to choose the most appropriate plan based on that day (Burridge et al., 2006). Another technique is to generate a patient-specific database that contain all plans delivered and if no plan fits the current case, the original plan will be adapted and then added to the patient database for future fractions $(\mathrm{Li}, 2011)$. The dose distribution from the original plan 
that performed on $\mathrm{pCT}$ is deformed onto the CBCT images to approximate the dose distribution for reoptimization process to match the 'anatomy of the day'. In addition, CBCT data can be used in retrospective IMRT dose reconstruction where the respective MLC log-files are retrieved and converted into fluence maps, then the dose is reconstructed on CBCT data with the regenerated fluence maps (Lee et al., 2008b). However, CBCT images generated using XVI on Elekta treatment units do not provide true Hounsfield units or CT numbers (Fotina et al., 2012).

In order to be able to perform CBCT-based ART, the CBCT images should be corrected. Compared with pCT, the image quality of $\mathrm{CBCT}$ is inferior due to the cone-beam geometry and associated scatter radiation, resulting in more image artefacts which make the correction of CBCT data more challenging. These image artefacts are described in detail in the rest of this chapter. However, different methods have been proposed for correcting CBCT data in order to achieve accurate dose calculation that is consistent with that calculated on pCT (for more detail see Section 1.2) (Yang et al., 2007, Boggula et al., 2007, Richter et al., 2008, Cheung et al., 2009, Fotina et al., 2012, Onozato et al., 2014, Dunlop et al., 2015, Held et al., 2015). The main aim of this thesis is to correct CBCT data and enable dose calculations to be performed quickly with the use of CBCT images as eliminating the need to rescan the patient and potentially avoiding additional dose.

\subsection{CBCT image quality}

Image quality can be defined as a measure of the accuracy with which the final image portrays the original object. In CT, it is therefore the accuracy with which the true HU, CT numbers or the actual linear attenuation coefficients of the materials are displayed in the image. Image quality can be expressed by some parameters such as image noise, contrast resolution and spatial resolution. In reality, a perfect representation of an object in the reconstructed images is impossible. Image quality is affected by image artefacts, which are any distortion in the image that is irrelevant to the object being scanned. In other word, an artefact in CT images is a systematic difference between the expected CT numbers and the CT numbers in the reconstructed images of an object. In addition, artefacts arise from several sources such as the X-ray source, the geometry of the imaging system, the image formation at 
the detector and the subject being scanned.

In the CBCT system, image quality is one of the more notable disadvantages because cone-beam geometry covers a large FOV in a single rotation with a large area detector, which results in an increased in the scattered radiation component. This affects the CBCT number or pixel value (gray scale), which shows the degree of Xray attenuation in CBCT images (in conventional CT, HU or CT number shows the degree of X-ray attenuation (see Chapter 1)). This leads to artefacts in the reconstructed image that influence image contrast, noise and pixel value accuracy. The most common and significant artefacts in $\mathrm{kV} \mathrm{CBCT}$ system are discussed in the following sections.

\subsubsection{Scatter artefact}

The interaction between soft tissue or bone and the incident X-ray beam is energy dependent. For CT imaging energies, Compton scattering takes place when the X-ray beam passes through a region of low atomic number $(Z)$ (see Chapter 4). In conventional CT, the X-ray source is collimated to only allow scatter generated from a thin axial region of interest to reach a single-row or multi-row detector. However, the X-ray is collimated to a wide beam in the CBCT system thus allowing scatter from the entire object volume to reach the detector. Large beam angles and a larger detector result in larger scatter as scattered photon at larger deflection angles are still more likely to be detected. These detected scattered photons are indistinguishable from the primary photons. The effect of scatter is that the beam appears to be more penetrating because the scattered and the primary radiation are detected together, instead of the primary radiation alone being detected. Thus the transmission in the projection measurement is overestimated and the true attenuation coefficient is underestimated, therefore degrading CBCT images. When the scatter varies slowly in the projection domain, the amplitude of the inhomogeneities towards the centre of the object is increased leading to slowly varying artefacts, called cupping or shading artefacts, which reduce the reconstructed pixel values. A sharp variation in the scatter in the projection domain, i.e. behind high density material such as bone or prosthesis, leads to sharply varying artefacts, called dark streak artefacts as shown in Figure 2.2 and Figure 2.3. These dark streak artefacts are seen particularly between 


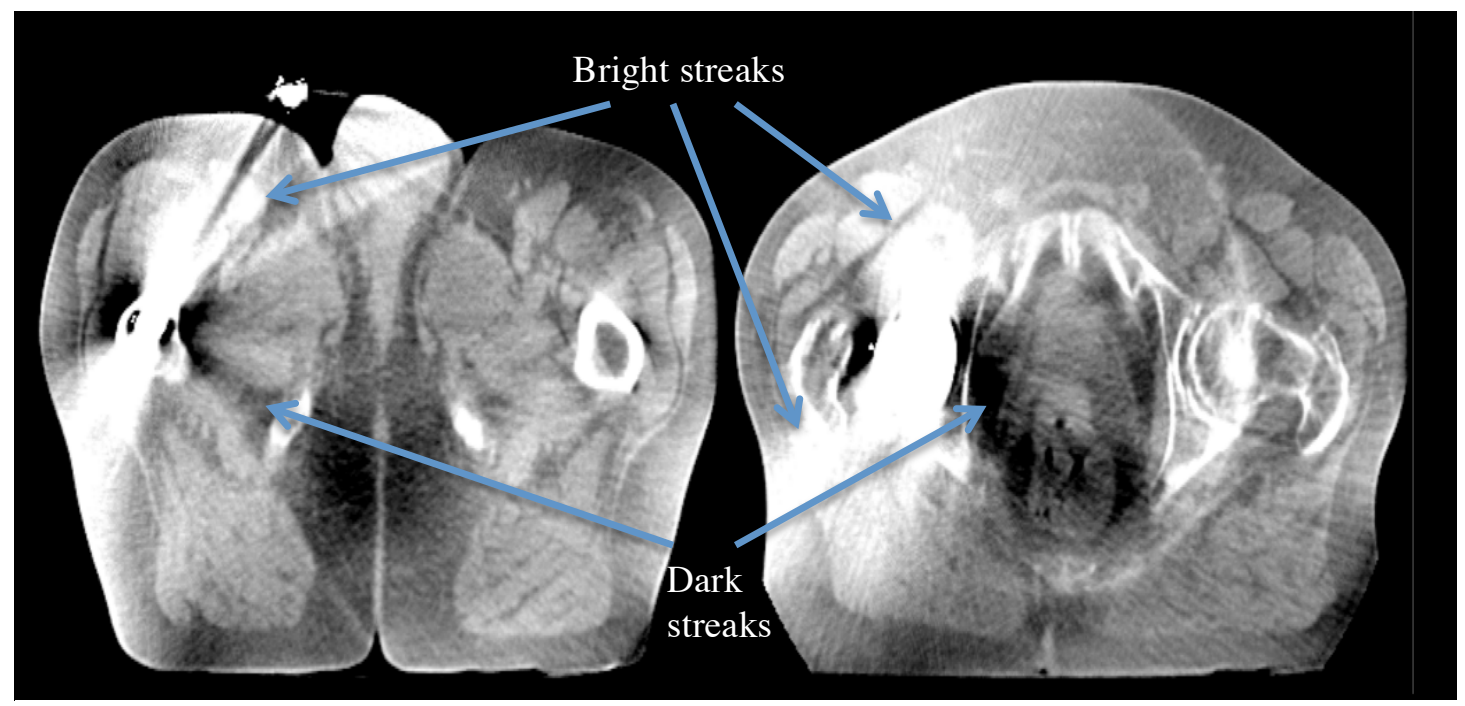

Figure 2.2: Example image illustrating X-ray scatter artefacts, due the presence of high density material, hip prosthesis.

heavily attenuating materials within the scanned object, leading to a large reduction in attenuation values.

In FDK reconstruction, applying the ramp filter to the projection data that are inconsistent along views with low or high X-ray scatter leads to bright streaks artefacts. In addition, the amount of scatter increases with FOV, collimations and object size, and varies with the acquisition parameters such as $\mathrm{kVp}$, number of projection and mAs. Furthermore, X-ray scatter degrades CBCT images by increasing noise that reduces the measured contrast between reconstructed tissues. Noise can be referred to as any stochastic variation in the CBCT pixel values. The best example of noise is quantum noise, which is a random distribution of individual X-ray photons incident on each detector element. In CBCT, quantum noise is high as the tube current value used is low, to avoid delivering high doses. This is considered to degrade the system's detective quantum efficiency (DQE), which is the fraction of X-ray photons contributing to the 3D image. DQE can be used to describe the image noise generated by X-ray scatter.

The scatter-to-primary ratio (SPR) is used to express scatter contribution and depends on FOV, filter used, object size and system geometry. In CBCT, SPR can be more than 1 showing that the majority of the detected photons have undergone at least one scattering event. An increase in SPR results in a proportional reduction in DQE, i.e. a SPR of 1 reduces the system DQE by a factor of 2 (Siewerdsen and Jaffray, 
2000).

There are several methods proposed to correct or minimize X-ray scatter artefacts. Changing acquisition parameters such as FOV, voxel size, number of projection and $\mathrm{kVp}$ can reduce the amount of scatter but also reduces the image quality and contrast. In addition, physical methods can be introduced to reject X-ray scatter at the detector that include a bowtie filter and an antiscatter grid. A bowtie filter (available in the XVI system, F1) improves image quality and reduces SPR, leading to improved CBCT pixel values accuracy and makes the best use of the dynamic range of the flat panel detector (Graham et al., 2007). A bowtie filter can be used to reduce X-ray scatter, and therefore, SPR, making it more uniform (see Section 2.2). For a midsize human torso, it has been shown that on a CBCT system SPR is around 2-3 and can be up to 8 in certain areas of the projection images that contain highly attenuating material (such as bone) even when using a bowtie filter (Zhu et al., 2009). It has been shown that using an antiscatter grid for $\mathrm{kV}$ images reduces scattered radiation but also absorbs part of the primary radiation, thus imparts a tradeoff in imaging dose, noise and artefacts (Siewerdsen et al., 2004, Shaw, 2014).

Even with when changing the acquisition parameters and the physical methods, the $\mathrm{X}$-ray scatter in CBCT can still significantly degrade image quality and pixel values in the reconstructed images. This suggests the need for correcting scatter artefacts by estimating the scatter contribution to the projection data, either using measurement, analytical approaches, MC simulation and modulation methods (Ning et al., 2004, Boone and Seibert, 1988, Jarry et al., 2006, Maltz et al., 2005). The analytical and MC approaches make some assumptions to increase computational efficiency which compromises the estimation accuracy. Measurement approaches can correct scatter artefacts but often result in high patient dose, as two scans per projection are required to compensate for the primary loss (Fan et al., 2015). Modulation methods tend to not correct scatter-induced high-frequency artefacts such as those caused by metal. However, all the correction methods mentioned above usually associate with inconvenient system hardware modifications, increasing the patient dose or increased computational time, preventing them from wide clinical use as an efficient correction method.

Even when using or applying the methods mentioned above to reduce scatter 
artefacts, it is still one of the major sources of image degradation in CBCT. The complicated and random nature of scattered photons makes the accurate rejection or estimation of the scatter contribution to the reconstructed images a very challenging task.

\subsubsection{Beam hardening}

As with all medical X-ray beams, the $\mathrm{kV}$ source in CBCT XVI uses a polyenergetic $\mathrm{X}$-ray spectrum. After passing through a patient, the lower energy X-rays are attenuated more than the higher energy X-rays, mainly because of the photoelectric effect. Therefore, as the polychromatic X-ray beam penetrates the object, the lower energies are absorbed, preferentially leading to a skew in the shape of the energy spectrum toward the higher energies. Consequently, the mean energy of the beam gradually increases (hardens) and is attenuated less as it penetrates the object.

The beam hardening causes artefacts in CBCT because the average energy of the beam from some projection angles is hardened more than from other angles. For example, the average energy of the X-ray travelling through the edges of the object is lower than those travelling through the centre of the object since there is less material for the beam to travel through. As the beam hardens, the effective attenuation coefficient within a large material length is lower than the expected. The reconstruction algorithm assumes that the attenuation is exponentially related to the object thickness, ignoring the energy dependence of the attenuation coefficients. Therefore, in the reconstructed image, the attenuation coefficient is underestimated, thus the centre of the object appears to be less dense than the edges, leading to an inconsistency in the CBCT pixel value of the same material. This leads to a dark area at the centre of the object, cupping artefacts (Figure. 2.3). The extent of the cupping artefact increases with object size and the location of the cupping is also dependent on the object's position relative to the isocentre.

Beam hardening also results in dark streaks between highly attenuating materials within the scanned object. When the beam crosses inconsistencies parts of a high attenuating material, such as bone, it leads to dark streak artefacts that join areas of this material in the reconstructed image. In addition, the reconstructed images may show bright streaks due to the application a ramp filter to inconsistent projection data. 


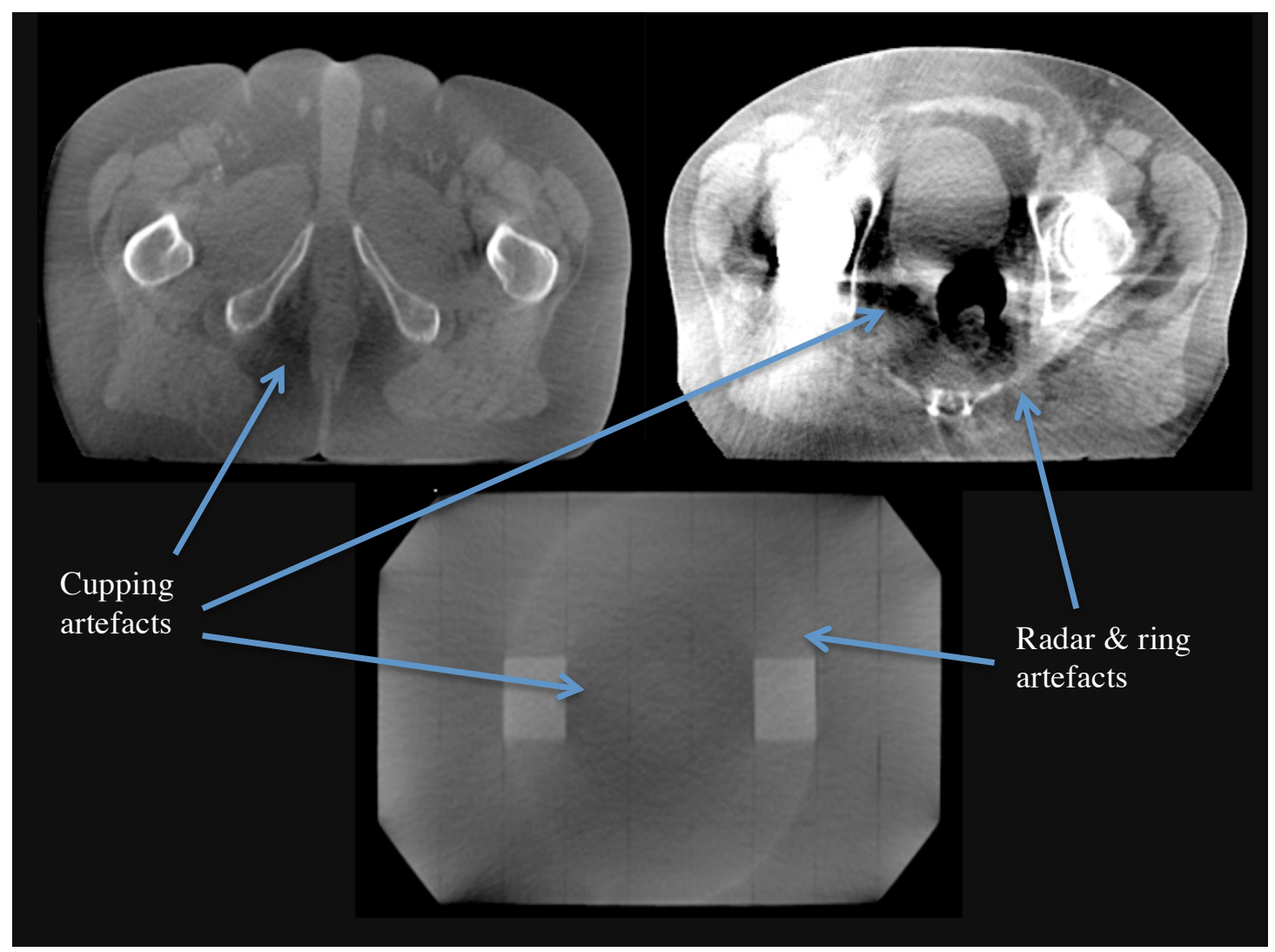

Figure 2.3: Example images illustrating beam hardening, between highly attenuating materials, radar and scatter artefacts.

In general, artefacts caused by beam hardening have similar characteristics to those caused by X-ray scatter, and it is therefore difficult to differentiate between them (Shaw, 2014). Compared with X-ray scatter artefacts, the magnitude of beam hardening artefacts tend to be smaller and more easily corrected (Bourland, 2012).

Beam hardening artefacts can be minimized using physical methods that include a bowtie filter and added beam filtration to flatten the X-ray fluence transmitted to the detector. However, using this method results in a reduction of the detector signal, thus increasing noise. More effective methods include nonlinear correction algorithms based on a water calibration for homogenous objects. For inhomogeneous objects, additional correction factors related to the energy dependence of the attenuation coefficients of the materials must be considered. Iterative correction algorithms can significantly correct beam hardening artefacts associated with more than one highattenuating materials with the advantages of improving image quality (Hsieh et al., 2000). 


\subsubsection{Ring artefact}

In CBCT, the FPD can be a source of image artefacts. Miscalibrated detector pixel lines/rows, elements or manufacturing defects in the FPD affect the quality of the reconstructed image, leading to artefacts. The shape of these artefacts is ring-like as the erroneous signal arises from a measurement that is from a fixed location on the detector, i.e. a dead or defective pixel. Thus a fixed defect is presented in the sinogram and overlapped in the backprojection at a fixed radius in the reconstruction, owing to the rotating motion of the detector during the acquisition. A ring artefact appears as a single or multiple concentric ring centered around the location of the axis of rotation and is presented in every axial slice of the 3D reconstruction (Figure. 2.3). Depending on the direction of the erroneous signal, the ring artefacts can be bright or dark (Shaw, 2014). Bright ring artefacts result in an increase in the reconstructed CBCT pixel values whilst dark ring artefacts result in a reduction in the pixel values. As mentioned in Section 2.2, the FPD can be shifted laterally to the centre of rotation to cover large volumes. Such a shift results in poorer uniformity with a ring artefact in the centre of the reconstructed images in the axial plane . In addition, beam hardening, or a change in detector exposure, causes pixel gain variation that leads to structured nonuniformities in projections and ring artefacts in CBCT images (Altunbas et al., 2014).

Ring artefacts can be minimized by sufficient offset-gain-defect detector calibration. Spatial variation in detector dark current and variations in the detector response to nonuniform X-ray should be corrected using offset and gain corrections, respectively. Using defect calibration can identify dead or noisy pixels that show erroneous offset or gain characteristics, these can then be filtered prior to filtered backprojection (Bourland, 2012). However, ring artefacts are not completely avoided using only experimental measures, thus methods to suppress them were developed. These methods include pre- and postprocessing techniques that are based on the sinogram processing and image space processing, respectively (Tang et al., 2001, Prell et al., 2009, Anas et al., 2011).

\subsubsection{Metal artefact}

The presence of metal implants within the patient such as prostheses can significantly 


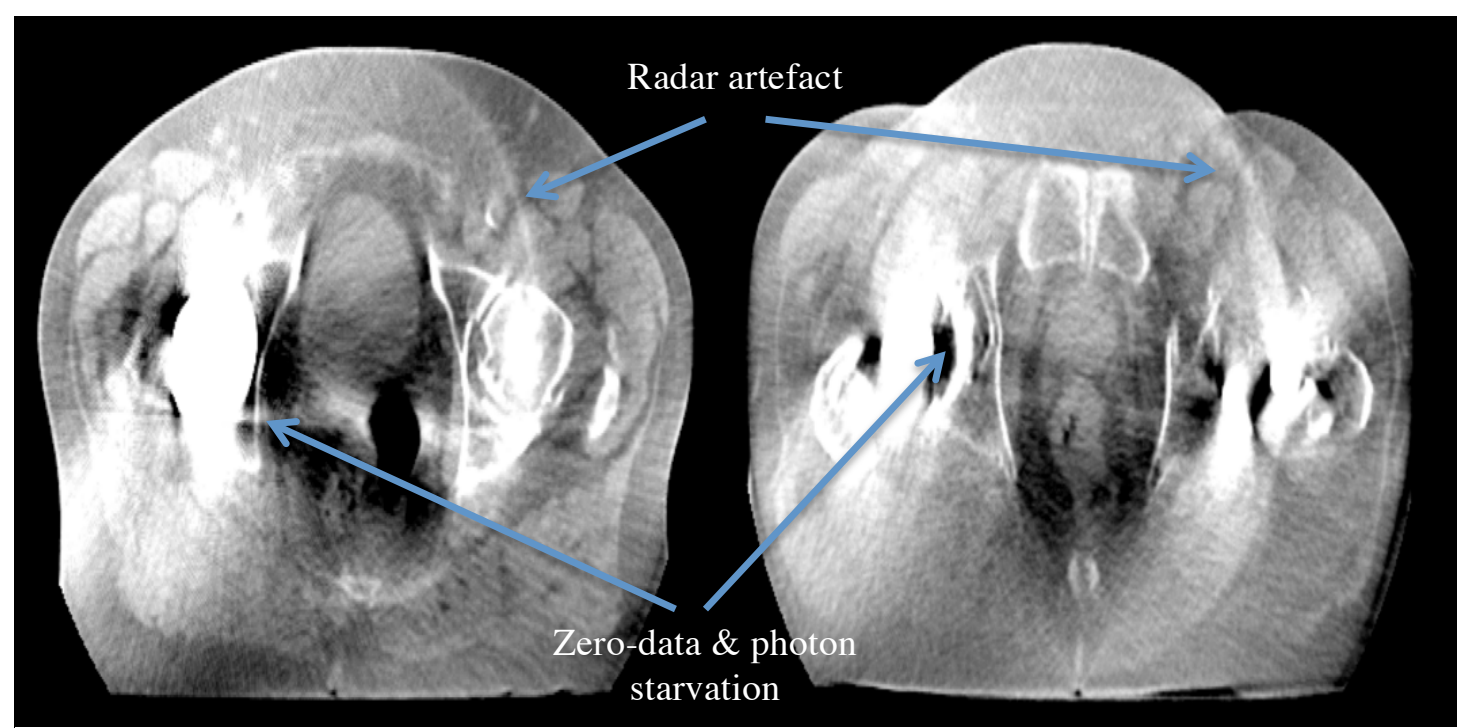

Figure 2.4: Example images illustrating metal artefacts due to the appearance of single (left) and double hip (right) prostheses.

degrade image quality by causing the so-called metal artefact, which is a combination of different effects (Pauwels et al., 2013, Bourland, 2012). These include scatter, beam hardening, quantum noise, zero data and photon starvation effects. The amount of scatter increases with the presence of a high attenuation material and it reaches the detector due to the large FOV of cone-beam geometry leading to cupping artefacts as mentioned in Section 2.4.1. After passing the metal object, the mean beam energy increases significantly due to the absorption of low-energy photons, as the photoelectric cross sections are very high with high $\mathrm{Z}$ materials (see Chapter 4). This beam hardening leads to a dark area at the centre of the metal object. If there are two prostheses within the scanned patient, the metal artefacts caused by the beam hardening effect appear as dark streaks between the metals with surrounding bright streaks. In addition, the presence of metal increases the graininess of the image due to the contamination of the detector signal.

The metal implant may also totally attenuate or absorb all the incoming photons along certain beam paths. Thus the detector signal, in such a situation, is zero leading to what is called zero-data artefacts. Due to the scatter and noise, the detector signal is very small but not zero in some cases. However, the zero-data artefacts appear as bright streaks radiating from the metal object corresponding to very high attenuation. It also appears, in combination with beam hardening, as dark areas between two metal objects or inconsistencies in part of one metal as shown in Figure 2.4. Furthermore, 
the presence of a metal object reduces the detector signal-to-noise ratio which leads, in combination with increased quantum noise, to a stochastic effect that is called photon starvation. This effect increases with the scanned object size due to the high attenuation. For prostate patients with two prostheses, for example, photon starvation leads to streaks between the two metals due to the increased noise caused by the lateral projections that suffer from strong attenuation through the two metals. As a result, metal artefacts caused by all the above effects lead to a significantly large variation in the CBCT reconstructed pixel values.

As metal artefacts are a combination of different effects, correcting of each effect separately can reduce metal artefacts, such as the beam hardening correction methods mentioned in Section 2.4.2. Photon starvation effect can be reduced by weighting noisy projection data using algorithms or by boosting X-ray intensity in projection using physical methods (Bourland, 2012). Furthermore, using multipass reconstruction-based or projection-based algorithms can minimize metal artefacts. In these algorithms, the projection data is restored to reasonable detector signal values. Using model-based iterative reconstruction algorithms can reduce metal artefacts but do not completely remove the artefacts due to the incompleteness of projection data (Williamson et al., 2002). For such algorithms, the knowledge about the shape and location of the metal object is required which is hard to get especially when the object lies behind a high density region such as bone. Auto-identifying the shape and location of the metal objects in the image space can be achieved by using an effective image intensity gradient threshold-based method (Wang and Xing, 2010). In this method, a binary image is obtained in such a way that the metal is 1 and all the other materials are 0 . Then an iterative algorithm is applied to the binary image by effectively utilizing the enormous difference in attenuation coefficients of metals and the surrounding materials.

\subsubsection{Motion artefact}

Another source of artefacts can be the motion of the scanned object during the image acquisition. In a conventional $\mathrm{CT}$, the gantry rotation takes around $0.3 \mathrm{~s}$ whilst the rotation time in a typical CBCT system mounted in a Linac is between 60 and $120 \mathrm{~s}$. For SFOV, the minimum gantry rotation required is $200^{\circ}$ which is used for small 


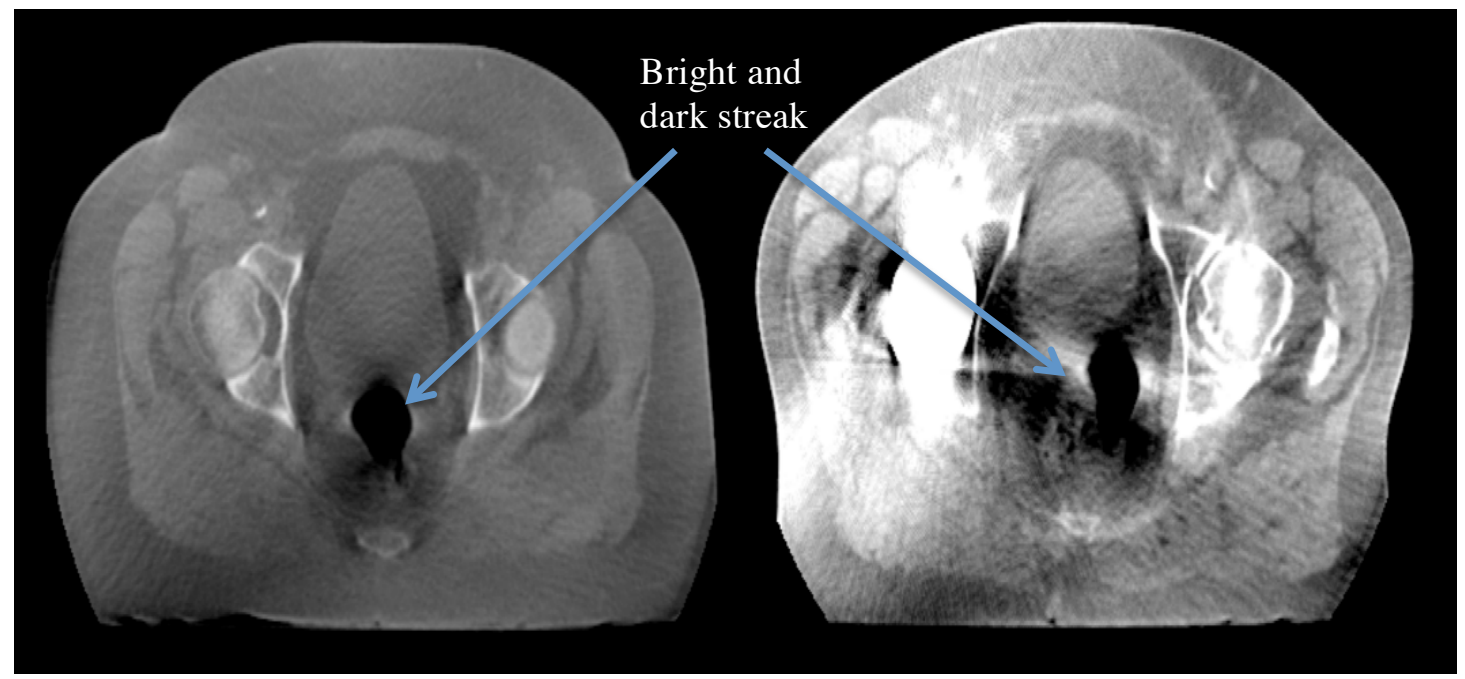

Figure 2.5: Example images illustrating motion artefacts.

scanned object such as lung, head and neck. For larger objects such as the pelvis, MFOV or LFOV, where the FPD is shifted, a full gantry rotation is required, thus requiring a longer acquisition time. Due to IEC requirements, the maximum gantry rotation speed is limited to 1 rotation per minute (International Electrotechnical, 1998). This longer acquisition time increases the possibility that the object moves during the acquisition, thus making $\mathrm{CBCT}$ images more prone to artefacts. Inconsistent projection data due to object motion affects the entire volume in the $3 \mathrm{D}$ CBCT. Such motion can be periodic, such as respiration, or random, such as gas in the rectum, that is difficult to avoid during acquisition. If the motion is constant, the motion artefacts appear as blurring in the direction of motion and if the motion is not constant it appears as streaks. It can also lead to streaks from high contrast structures such as air cavities. For example, moving gas in the rectum can cause bright streaks at the rectum wall and dark streak around the rectum. Figure 2.5 shows bright streak artefacts caused by a small motion of gas within the rectum. For prostate cancer delineation, in some cases, such an artefact reduces the visibility of the prostate.

Motion artefacts caused by periodic motion can be reduced by using gating techniques that include prospective and retrospective gating methods (Sonke et al., 2005, Li et al., 2005). Motion artefacts caused by random motion are minimized by using positioning aids and applying appropriate protocols. For prostate cancer patients, using a dietary protocol decreases motion artefacts and improves CBCT image quality (Smitsmans et al., 2008). Furthermore, appropriate quality assurance of the mechanical stability of the XVI system is required as the motion of the $\mathrm{kV}$ source 
and detector can lead to motion artefacts that are minor compared with those caused by patient motion.

\subsubsection{Cone-beam and truncation artefact}

As mentioned in 2.2.3, a circular source-detector geometry in the CBCT system results in an incomplete sampling of a volume, leading to cone-beam artefacts. Such geometry violates the Tuy-Smith condition for exact reconstruction, which says that every plane passing through a point in the reconstruction must intersect the circular trajectory. Only voxels in the central axial slice are meet with this condition and they are accurately reconstructed. Any voxels that are away from the central plane violate the condition and are not reconstructed accurately, and are thus subject to cone-beam artefacts. This is due to the incomplete sampling of the full 3D Fourier domain from a circular trajectory that is then sampled by a 3D filtered back-projection. As the distance from the central plane increases, thus increasing the cone angle, the degree of incompleteness increases. The artefacts appear as a reduction in the CBCT pixel values. Cone-beam artefacts can be minimized by using exact analytical reconstruction algorithms or non-circular source-detector trajectories (Pearson et al., 2010, Davis et al., 2014).

Even though the FPD can be shifted laterally to cover a large FOV, it is often insufficient to completely cover large objects or lateral extent of the patients, such as the pelvic region, thus obtaining incomplete projection data. This incompleteness results in so-called truncation artefacts that appear as bright rings about the periphery of reconstruction and dark cupping near the centre of reconstruction, caused by a reduction in the $\mathrm{CBCT}$ pixel values in the centre of the reconstructed images and an increase in the periphery regions. If there is a highly attenuating material within the FOV such as prostheses, truncation artefacts appear as bright streak in some projections. However, truncation artefacts can be corrected by extrapolating the projection data at truncated edges or estimating the missing data from the prior CT scan (Bourland, 2012).

\subsubsection{Other artefacts}

The FPD shows some residual signal frame-to-frame leading to image lag artefacts. 
Due to the increased charge trapping in the amorphous-silicon semiconductor of the FPD, the signal read in a given projection may have been already generated in the previous projection. Such an effect leads to an azimuthal comet artefact behind high contrast structures that depends on the distance from the centre of reconstruction and the degree of image lag. Similarly, a change in the detector gain or sensitivity is dependent on previous exposures, leading to an effect called image ghosting. Both image lag and ghosting effects cause the so-called radar artefacts that appear as a bright-shaded region (ring-like) primarily about the periphery of a large asymmetric object (Figure. 2.3 and Figure. 2.2). For example in a pelvic region with prostheses, there is a rapid change in the exposure to the FPD from frame to frame, receiving high exposure then followed by low exposure due the strong attenuation of the metal. Such an artefact can be minimized by measuring the lag characteristics and subtracting the effect of the previous projection from the current one (Mail et al., 2008).

Another kind of artefact that degrades CBCT image quality is called the aliasing artefact. Due to the cone-beam geometry, in each projection the number of recorded rays traversing a voxel depends on the location of the voxel relative to the detector. The closer the voxel to the detector the lower the number of rays. This leads to line patterns in the reconstructed CBCT images. In addition, approximating the length of each ray traversing the voxels by linear interpolation during backprojection in the FDK algorithm may result in aliasing artefacts (Schulze et al., 2014). Thus more complex backprojection techniques, or better interpolation methods can reduce aliasing artefacts (De Man and Basu, 2004).

Although CBCT images may display any or all of the above artefacts, they are an improvement over 2D imaging for radiotherapy on-treatment verification and aid treatment. $\mathrm{CBCT}$ is an important part of image guided radiotherapy. Due to its volumetric anatomical information, Wong et al (2014) wrote that "The highly localized nature of RT predisposes its close tie to advances in imaging. The community has long relied on some form of imaging to verify patient setup and beam placement. But as treatment methodologies evolve with technological advances, cone beam computed tomography (CBCT) has rapidly become the main enabling technology for image guidance since its commercial introduction in 2005. The advent of CBCT heralded our present era of image-guided radiation therapy (IGRT), and 
perhaps more appropriately, the era of volumetric or three-dimensional (3D) IGRT" (Wong et al., 2014).

However, if CT or CBCT images are used for dose calculation, the dose calculation accuracy depends on the accurate representation of CT or CBCT number. In addition, the dose calculation accuracy depends on the dose calculation algorithms. In the next chapter, simple dose calculation algorithms as well as those used in this thesis will be presented. 
Chapter 3 Dose calculation algorithms 


\subsection{Introduction}

The aim and at the same time the greatest challenge of radiotherapy is to give a tumoricidal dose to the tumour and to spare healthy tissues. Ideally, more than $95 \%$ of the PTV dose should cover the tumour. Organs at risk should receive much less dose depending on the individual organ. Much effort has been made to improve technologies in order to achieve this goal. As mentioned in Chapter 1, a typical radiotherapy treatment is a complex process and includes a number of different phases (i.e. simulation, planning phases) that are all taken successively in order to obtain an optimal process and to improve treatment outcomes. Each phase of these contains inherent uncertainties associated with the dose delivery. It has been stated in ICRU report 83 (International Commission on Radiation Units and Measurements) that the uncertainty of dose delivery to the patient should be less than $3.5 \%$ (ICRU, 2010). The uncertainty should include systematic and random errors, which can be reduced by acquiring CBCT images (see Section 2.3). This in turn requires that the accuracy of dose calculation algorithms implemented in a commercial TPS should be within $\pm 2 \%$ (Papanikolaou et al., 2004).

Currently, most of the TPS algorithms cannot reach that level of accuracy, especially in situations where density inhomogeneties or atomic number variations are present (e.g. tissue/metallic prosthesis interfaces) (Ding et al., 2005, Aarup et al., 2009). This is mainly due to the fact that the calculation accuracy is balanced by the calculation speed to be fast enough for clinical use. The inhomogeneity corrections methods involved fail to predict the lateral transport of the secondary electrons and the separation of primary and scatter dose components (Knöös et al., 2006). In addition, accurate dose calculation does not only rely on accurate calculation algorithms but also depends on the accurate representation of HU or CT number and accurate HU-ED calibration.

There is no consensus on a classification methodology for dose calculation algorithms but in general use there is type-a and type-b algorithm (see Section 3.4) (Knöös et al., 2006, Mayles et al., 2007). A suggestion was made by Mackie et al. (1996), who distingushed between correction-based algorithms, where the dose distribution in water phantom is computed and manipulated for the patient characteristics, and model-based algorithms, where the dose in the patient is 
computed directly. Battista et al. (1997) suggested distinguishing between broad beam methods, where the primary and scatter dose components are not distinguished, and superposition methods, which calculate the primary and scatter radiation separately. However, based on the inhomogeneity corrections for photon beams, dose calculation algorithms can be categorized into four groups, (1) those that only take into account the variation in density between the radiation source and the point of calculation and thus treating all dose as primary (e.g. equivalent path length), and (2) those that correct for the primary and scatter dose (e.g. equivalent tissue-air ratio), and (3) those that account for the primary and scatter components and electron transport by macroscopic means (e.g. convolution/superposition method, such as pencil beam and collapsed cone, which OMP has), and (4) those that account for primary and scatter component and electron transport by microscopic means (e.g. Monte Carlo) (Keall, 1996, Papanikolaou et al., 2004). The accuracy of the dose calculation increases from method (1) to (4) as the number of simplifications reduces but the calculation times grow by a large factor. These methods have been drawn in what follows.

\subsection{Effective path length method}

The effective path length (EPL) method is considered to be the simplest method to correct for an inhomogeneity where a simple correction factor based on the radiological depth is used to modify depth dose data. Effective or radiological depth is the thickness of water-equivalent tissue that would give approximately the same attenuation for photon beam by the same amount as the actual tissue along the ray line between the source and the calculation point. The effective depth, $d_{\text {eff }}$, of a geometrical actual depth, $d$, is determined by summing the product of thickness $t_{i}$ and density $\rho_{i}$ of the material that each ray passes through to reach that point (Milan and Bentley, 1974)

$$
d_{e f f}=\sum_{i=1}^{d} t_{i} \rho_{i}
$$

The dose to this point is then obtained by multiplying the dose in water at $d_{e f f}$ by an inverse square correction to account for the difference between the effective depth and the geometrical depth, 


$$
D(d)=D\left(d_{e f f}\right) \times \frac{\left(S S D+d_{e f f}\right)^{2}}{(S S D+d)^{2}}
$$

where $S S D$ is source to surface distance. This method is only valid for the primary component when only Compton effects with peripheral electrons are considered assuming that the electron fluence mediated between the source and the calculation point is the same, charged particle equilibrium (CPE). The result of applying this correction in high density media gives a lower predicted dose than if the medium were unit density (water). On the other hand, in low density media, the result gives a greater predicted dose than for unit density (Metcalfe et al., 1993). This is mainly due to the fact that this correction considers only modification of the primary dose and dose not attempt to consider scattered photons and secondary electrons where the electron disequilibrium occurs at media interfaces with different material densities.

\subsection{Equivalent tissue-air method}

Tissue-air ratio (TAR) method is slightly more accurate technique than the equivalent pathlength method. It is defined as the ratio of dose at a given depth, $d$, in tissue (or water) to the dose in air at the same the depth. The TAR correction, $C$, applied to the dose is

$$
C=\frac{T A R\left(d_{e f f}, A_{d}\right)}{T A R\left(d, A_{d}\right)}
$$

where $A$ is the field size at depth $d$ and $d_{\text {eff }}$ is the effective depth.

Even thought TAR method provides a slightly better accuracy than EPL method, both methods are equivalent at some distance from inhomogeneities where both do not consider the scatter radiation (Batho, 1964). Sontag and Cunningham (1978) developed TAR method to introduce the so-called equivalent tissue-air ratio (ETAR), which is the first algorithm that accounts the scattered dose. Compared with TAR where only the depth is scaled, ETAR method uses the effective depth, $d_{\text {eff }}$, and an effective density, $\rho^{\prime}$, to scale the field size in proportion to the lateral electron transport and associated photon scattering for each calculation point, $d$ (Sontag and Cunningham, 1978). The ETAR relies on O'Connor theorem which states that the 
dose to a point in a homogenous non-unit density medium is the same as in a unit density provided the geometric depths and beam radius are scaled inversely with density (O'Connor, 1957). If the density information is obtained from voxels in a CT image, the effective density is obtained by performing a weighted sum of the density of each voxels surrounding, $d$, and calculated as

$$
\rho^{\prime}=\frac{\sum_{i j k} \rho_{i j k}, W_{i j k}}{\sum_{i j k} W_{i j k}}
$$

where $W_{i j k}$ are the weighting factors for each voxels in the irradiated volume that contributed to the scatter dose and $i j k$ are the total number of voxels.

This is a $3 \mathrm{D}$ summation over the entire irradiated volume, which is too time consuming, thus impractical to be used routinely in clinic. To overcome this limitation, the CT scans are coalesced into a single slice positioned at an effective distance from the calculation point so the effective density is obtained from the $2 \mathrm{D}$ summation. This slice is called, virtually, the effective slice or the effective scatter slice which reduces this method to a 2.5D method (Sontag and Cunningham, 1978). The field size is then scaled by multiplying the beam radius by the effective density, $\rho^{\prime}$,

$$
C=\frac{T A R\left(d_{e f f}, A_{d e f f}\right)}{T A R\left(d, A_{d}\right)}
$$

The result of applying this correction gives an overestimation of the predicated dose in low density media, such as lung, as the primary and scatter dose components rely on TAR measurements obtained under equilibrium conditions in unity density (water), thus in a heterogeneous medium this method does not account for electron disequilibrium. For low energy photons (in keV range), TAR assumption can predict the electron disequilibrium where the range of the secondary electron is small. Thus satisfying O'Connor theorem, in a homogenous non-unity density as well, where the effect of the atomic number variations is neglected and assuming that the secondary electrons travel in straight line. On the other hand, at high energy (in $\mathrm{MeV}$ range, radiation therapy) and low density region where the range of electrons is increased 
from that in water and the sum of electrons scattered from the central axis of the beam are not balanced by the sum of electrons scattered into the central axis of the beam, this assumption fails to predict such a situation (Carrasco et al., 2004). Since the secondary electrons do not travel in straight lines especially at medium interfaces, the dose is overestimated at the calculation point and underestimated lateral spread. In addition, the weighting factor, $W_{i j k}$, used to determine the effective density is assumed constant with respect to the depth, only a function of the irradiated area, and the media between the effective scatter slice and the calculation point is assumed to be water (Cedric and Wong, 1993). Therefore, the scatter component is considered as a whole with implicit ray tracing for the scatter dose calculation through the inhomogeneous region.

\subsection{Convolution/Superposition method}

All methods mentioned so far are based on a large number of input data as such dose profile curves and depth dose curves for several field sizes of each available beam energy that is calculated in water and then corrected for the patient characteristics. These are known as correction-based methods. Thus, it is assumed that the electron equilibrium exists even in inhomogeneity and build-up regions. This is not true in photon beams where the range of the secondary electrons produced is long and the scatter contribution becomes less important, such as for MV treatment beam or in inhomogeneous regions. In this case, electron transport cannot be ignored and the change in the photon fluence caused by an inhomogeneity is not proportional to the change in dose (Mackie et al., 1985a). The superposition method, on the other hand, does not rely on measured dose data and is a model-based method where the interaction in the patient is computed directly taking into account the patient characteristics and the beam modifiers. Not only does superposition account accurately for primary and scattered photons, but it also accounts for the range of secondary electrons, and hence the situation of electron disequilibrium occurring at media interfaces is modeled (Mackie et al., 1996).

TERMA is the total energy released to unit mass by the photon beam at each interaction site and depends on the primary photon fluence. In a convolution calculation, the pattern of energy deposition about a primary photon interaction site, 


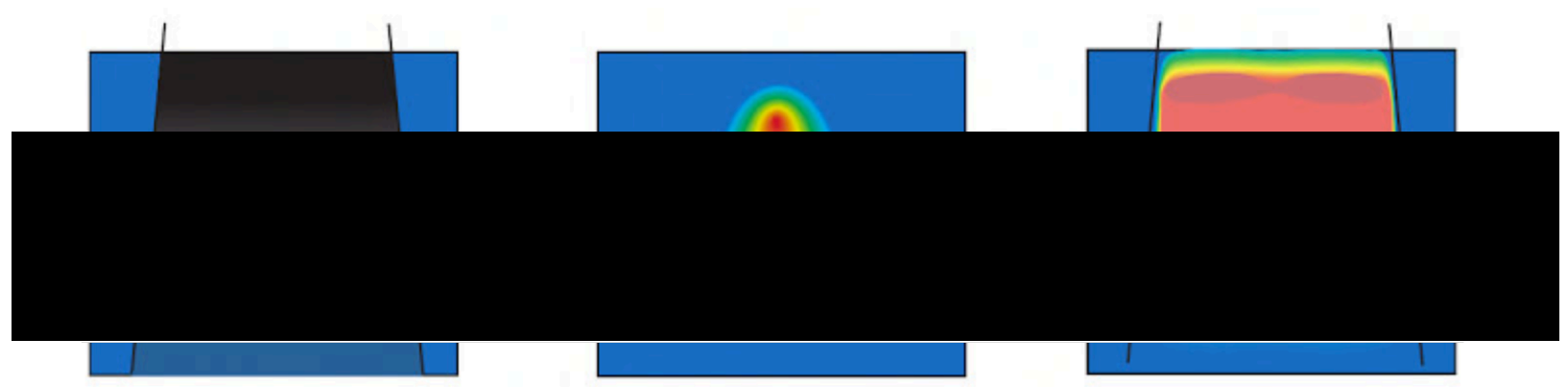

Figure 3.1: Convolution method performed with TERMA and kernel to obtain dose distribution (McDermott, 2016).

per unit TERMA (known as kernel or dose spread function or energy deposition kernel) is convolved with TERMA to obtain the dose distribution (Figure 3.1) (Ahnesjö, 1989, Mackie et al., 1985b). For inhomogeneities, the kernel used can also be scaled to account for the density variations. In this case, the convolution method is referred to as the superposition method.

These kernels are calculated in water using Monte Carlo calculation (see next section), which can then be adjusted to fit dose distributions for individual clinical beams, or using analytical calculation, constructed using multiple scattering theory (Papanikolaou et al., 2004). Kernels can be split into primary and scatter kernels as they require knowledge about the appropriate energy spectrum and fluence of the primary and secondary photons as well as the photon and electron contamination component. The primary kernel is defined as the three dimensional distribution of energy deposited by secondary particles from the primary interaction site. Scatter kernel is defined as the energy deposition by secondary particles generated by scattered photons. However, there are two common elementary energy deposition kernel classes for model-based algorithms that are implemented in current clinical treatment planning systems, pencil beam and point kernels (Ahnesjö, 1989, Mackie et al., 1988). At Singleton hospital, where this study takes a place, the treatment planning system used, OMP TPS, supports both kernel classes. Pencil beam kernel is referred to as a type-a, where lateral electron transport is modelled as for homogenous media, whilst point kernel is referred to as type-b, as collapsed cone, where the lateral electron transport is considered in heterogenous media in an approximate way (Knöös et al., 2006).

Pencil beam kernel was used in most TPSs due to the fact that it is fast and accurate 
enough for dose calculation. It describes the three-dimensional dose distribution of an infinite monoenergetic photon beam in water equivalent media. It uses the integration of point kernels along a line of a beam in a phantom to obtain a pencil beam type dose distribution, thus assuming all interaction points of the photons are on the central axis of the pencil beam. The dose from the pencil beam is precalculated and then scaled according to the relative electron density along the ray:

$$
D(x, y, z)=\iint \Psi\left(x^{\prime}, y^{\prime}\right) \frac{p}{\rho}\left(x-x^{\prime}, y-y^{\prime}, z\right) d x^{\prime} d y^{\prime}
$$

where $\mathrm{D}$ is the dose at a point $(\mathrm{x}, \mathrm{y}, \mathrm{z}), \Psi$ is the entrance energy fluence and $\mathrm{p} / \rho$ is the dose distribution of a pencil beam scaled with density $\rho$. The two dimensional integration is limited to the field area (Ahnesjö and Aspradakis, 1999, Krieger and Sauer, 2005).

In a homogenous water-like medium, the equation (3.6) is a true convolution method as the kernel can be assumed spatially invariant thus the calculation time can be speeded up by means of Discrete Fourier Transform (DFT) techniques (Ahnesjö and Aspradakis, 1999). In a heterogeneous medium, this assumption is no longer true and the mathematical solution is a superposition. The density variation is accounted for by the EPL method for the primary dose. For the scatter dose (including secondary electrons), the dose is calculated using the geometrical depth and then multiplied by a 1D convolution correction factor, which is calculated using the radiological depth to account for heterogeneities along the pencil beam (Ahnesjö et al., 1992).

As a result, this method cannot model accurately the scatter and electron transport in a heterogeneous medium (Wieslander and Knöös, 2000). The electron variations perpendicular to the pencil beam axis are not accounted for since it is based on a 1D correction factor for the scatter photons. As a consequence of generating point kernels in water, pencil beam calculates dose to water thus the secondary electron pathlentgh will not be accurate in lower or higher density medium where electronic disequilibrium occurs. Even though the PB algorithm uses a set of monoenergetic kernels over the effective spectrum to compose polyenergetic kernels, scaling these kernels for different densities must be done to reflect the secondary electrons tracks. In low density medium, such as lung, the $\mathrm{PB}$ algorithm can result in an overestimation 
of the dose on the central beam axis by $12 \%$, ignoring the the enhanced lateral scattering of secondary electrons (Oelkfe and Scholz, 2006). The overestimation increased with increasing beam energy showing magnitude similar to that found in the conventional correction-based algorithms (see Section 3.2 \& Section 3.3). However, despite these limitations, PB algorithm was the most widespread in clinical TPSs as it provided an acceptable compromise between accuracy and speed for a wide range of tumour sites. For example, it is used for pelvic region where there are fewer inhomogeneties or atomic number variations. For IMRT, where there are many beam segments used and increasing the speed of calculation time is desired, PB algorithm is well suited for such a technique and provides the best balance between dose accuracy and calculation times (Oelkfe and Scholz, 2006, Mayles, 2007).

However, with ever-increasing computer power, the more accurate type-b algorithms are now of comparable calculation speeds and are therefore becoming the standard algorithm for most modern TPSs such as Raystation (V4.7, RaySearch Laboratories, Stockholm, Sweden). Point kernel is referred to as a type-b and describes the distribution of absorbed energy in water from a photon interaction site as a function of direction and distance, where the primary photons are forced to interact at specific point, i.e. at the central voxel (Ahnesjö and Aspradakis, 1999). For dose calculation, TERMA is calculated then convolved with a kernel that combines the primary and scatter contributions over the entire volume:

$$
D(x, y, z)=\iiint T\left(x^{\prime}, y^{\prime}, z^{\prime}\right) K\left(x ; x^{\prime}, y ; y^{\prime}, z ; z^{\prime}\right) d V^{\prime}
$$

where $\mathrm{D}(\mathrm{x}, \mathrm{y}, \mathrm{z})$ is the dose at the calculation point and $\mathrm{T}\left(x^{\prime}, y^{\prime}, z^{\prime}\right)$ is TERMA distribution around the calculation point. $\mathrm{K}\left(\mathrm{x} ; \mathrm{x}^{\prime}, \mathrm{y} ; \mathrm{y}^{\prime}, \mathrm{z} ; \mathrm{z}^{\prime}\right)$ is the point kernel or energy deposition point kernel that represents the energy released from the interaction volume $\mathrm{dV}^{\prime}$ and deposited at the calculation point. The three-dimensional integration represents the entire volume (Ahnesjö, 1989, Ahnesjö and Aspradakis, 1999, Mayles et al., 2010).

For a homogenous medium, the shape of the kernel can be assumed to be transitional invariant, thus the kernel is changed to $\mathrm{K}\left(\mathrm{x}-\mathrm{x}^{\prime}, \mathrm{y}-\mathrm{y}^{\prime}, \mathrm{z}-\mathrm{z}^{\prime}\right)$ as only a function of distance between the interaction and calculation point. For an inhomogeneous medium, the density scaling that relies on rectilinear scaling based on 
O'Connor theorem is applied directly to the point kernel (see Section 3.2). Therefore, scaling the kernel in all directions is obtained by multiplying all dimensions by the effective density, which is used to change secondary particle contributions. It is assumed that electrons travel in straight lines from the interaction point to the calculation point. In inhomogeneities, such as hip prostheses, this leads to a significant dose calculation uncertainty (Spirydovich et al., 2006). However, since the calculation is no longer a convolution in an inhomogeneous medium (the kernel is not spatially invariant), the main problem of superposition is that the computation time is too long even if it is performed with an advanced hardware technology, thus many approximations have been proposed to reduce the computation time (Schlegel et al., 2006). One of these approximations is called collapsed cone (CC) and introduced by Ahnesjö (1989).

The CC algorithm (also called run-length raytracing) is a full three-dimensional convolution/superposition and uses an analytical kernel that is subdivided into a number of cones, with each cone radiating away from the interaction site and contributing to a fraction of the solid angle around the interaction point that together constitute a $4 \pi$ solid angle (shown in Figure 3.2) (Reckwerdt and Mackie, 1992, Ahnesjö, 1989). The electron transport and scattered photons that occur inside each cone is collapsed on the central axis of the cone, hence the name. The energy released is then calculated, for primary and scatter components, only along that axis (Ahnesjö, 1989). In a CT image, each cone axis is corresponded by one ray that crosses each voxel, which picks up energy released from points along the ray and later on deposits at dose deposition points along the ray according to the density variation. The kernel is then a number of energy deposition rays. Therefore, it reduces the calculation number from $\mathrm{N}^{6}\left(\mathrm{~N}^{3}\right.$ number of deposition voxels, from $\mathrm{N}^{3}$ number of interaction in 3D) to $M . N^{3}$ where $M$ is the number of cones. Decreasing $M$, less voxels are intersected by cones, would decrease the calculation time but decrease the accuracy as well. Voxels that are not assigned with energy deposition are compensated from other cones, thus with increasing distance from the kernel origin the accuracy decreases.

As a result, the $\mathrm{CC}$ algorithm can model the contribution from single scattered photons to the dose whilst it approximates the multiple scatter contributions (Krieger and Sauer, 2005). Compared with PB algorithm that calculates dose to water, CC 


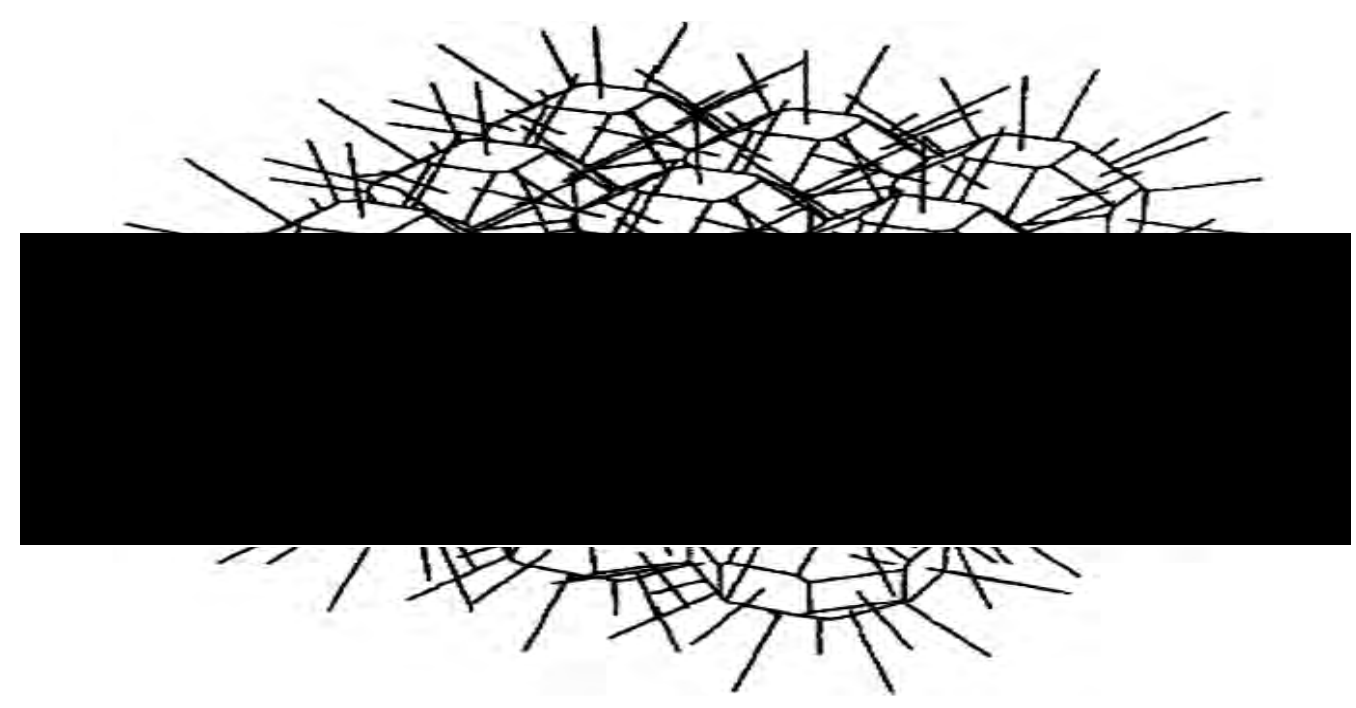

Figure 3.2: An example of CC, 13 cone axes representing 26 possible directions of particle transport cover a lattice made of $3 \times 3 \times 3$ voxels (Ahnesjö, 1989).

algorithm, type-b, calculates dose to the medium specified thus provides more accurate dose calculation in heterogeneous media. Other implications are, since it calculates dose to medium, the $\mathrm{HU}$ of the medium must be accurate and the comparison with dose to water plan is not direct. In addition, it helps the comparison to $\mathrm{MC}$ results. However, using rectilinear scaling for secondary electron transport overestimates lateral scattered dose in a layer beyond high-to-low density interface, especially for large field sizes. This is because the rectilinear scaling does not account for the multiple scatter events, which is the largest contribution to lateral electron spread, providing wider electron spread (Woo and Cunningham, 1990). In low density region (i.e. lung) and high energy beam (15 MV), this can overestimate the dose by up to $6 \%$ outside of the field (Nisbet et al., 2004). In high density inhomogeneities, such as hip prostheses, it underestimates the dose in regions just downstream of low-tohigh density interfaces due to primary interactions before the interface and the increased backscatter caused by the metallic hip, which is not modeled at all (Spirydovich et al., 2006). For dose due to recoil electrons generated by primary photons, the rectilinear scaling is valid but for subsequently scattered photons and their recoil electrons the rectilinear approximation is no longer valid. This is not an issue in the pelvis region but in head and neck region. Therefore, the $\mathrm{CC}$ algorithm cannot accurately account for electronic disequilibrium. However, the CC, whilst it has limitations, is amongst the most accurate TPS algorithms currently widely available in commercial TPSs. 


\subsection{Monte Carlo method}

Monte Carlo (MC) is an indirect numerical solution to the Boltzmann transport equation describing the interaction of a continuous field of particles and a direct simulation of the physical processes occurring in radiation transport and proven to be the most accurate method for dose calculation (see Chapter 4) (Andreo, 1991, Seco and Verhaegen, 2013). Similar to the convolution/superposition method, the MC method does not rely on measured dose data but it calculates dose distribution directly from the known characteristics of the incident beam. In MC, there is no approximate scaling to correct dose within heterogeneous media and the electron transport is modeled but not on an event by event basis as there are some approximations made (see Chapter 4 and 5) (Seco and Verhaegen, 2013).

Unlike convolution/superposition, MC does not account for the primary and scatter components and electron transport by macroscopic means but rather microscopic, considering groups of particles. By the sampling of random variables from the probability distributions obtained from an understanding of the physics, charged particle transport can be simulated and thus the dose deposition. Random number techniques are well suited to the simulation of particle transport since probability in the quantum mechanical interaction of the particles is implicit. If the linear accelerator geometry and components as well as the composition of the patient are provided accurately, and enough particles are used in the simulation to achieve an acceptable level of sampling or statistical errors, MC can calculate the dose deposition accurately even in dosimetric situations where analytical techniques cannot handle. A large number of particles must be simulated (called "histories") to reduce the statistical errors of the output due to the stochastic nature of the interactions, especially for the photon beams where the mean free path is long and consequently there are a small number of interactions. The relationship between number of particles $(n)$ and statistical errors is $1 / n^{1 / 2}$. Thus, MC is capable of providing results as accurately as statistical errors will permit (Rogers and Bielajew, 1988, Andreo, 1991).

The most common MC code used in radiotherapy is EGS (Electron Gamma Shower) though there is a number of other codes, such as MCNP (Monte Carlo NParticle), ETRAN (Electron Transport), PENELOPE and GEANT codes (Nelson and Hirayama, 1985, Briesmeister, 1986, Berger and Seltzer, 1973, Baro et al., 1995, Brun 
et al., 1978). In this study, a version of EGS called EGSnrc code is used which consists of user-written code and EGS system code (Kawrakow and Rogers, 2000). The user-written code contains the setup parameters of the simulation that are used to initialise particle histories, define the geometry and to record the result, such as dose deposition, particle location, direction, type and energy. The EGS system code contains subroutines that are independent of the user setup to transport each particle through the given geometry and the medium using PEGS file (this contains physical properties such as energy dependent photon attenuation coefficients, electron stopping powers and mean free paths).

As a result, the $\mathrm{MC}$ algorithm provides the most accurate dose calculation compared with the current clinical dose algorithms. It simulates each individual particle taking into account the medium density variations, such as lung and surface irregularities, thus calculating the dose to medium. MC models the multiple scattering explicitly whereas CC method is an approximation. The accuracy of MC calculation can achieve $2 \%$ in dose calculation compared with the present TPS algorithms where the accuracy varies between 3-8\% (Francescon et al., 2003). In spite of the accuracy, the main limitations of MC method are the simulation time and the requirement of the exact geometry of the treatment head machine, which may not be easily available to users. For example, it takes 2.1 hours to simulate $10 \times 10^{6}$ particles on a simple water phantom (size of 57 x 57 x 70 voxel) running on a OS X 10.8 with $3.0 \mathrm{GHz}$ Dual-core Intel Core i7 processor, turbo Boost up to $3.5 \mathrm{GHz}$ and a $\mathrm{RAM}$ size of $8 \mathrm{~GB}$. Therefore, these limitations had prevented the use of pure MC calculation in the clinical routine for many years. Moore's law states that the capacity of memory chips double every 18 to 24 months (Thornton and Rex, 2012). This might reduce the problem of MC calculation time in near future. However, with the arrival of modern fast computers, many efforts have been made in reducing the calculation time to a clinically acceptable level, i.e. running over night to achieve accuracy between 3-8 \% using a cluster (see Chapter 4). Some of these are the implementation of variance reduction techniques and the use of distributed computing techniques (Verhaegen and Seuntjens, 2003, Downes et al., 2009). Such techniques allowed the implementation of MC in commercial TPS such as Elekta Software XiO (XVMC), Varian Eclipse (eMC), and Nucletron Oncentra MasterPlan (VMC++) (Fippel, 1999, Neuenschwander et al., 1995, Kawrakow, 2001). 
In this study, EGSnrc code will be used to model the Elekta Synergy linear accelerator. In the next chapter, the general MC simulation concepts as well as MC transport of photons and electrons will be reviewed. 
Chapter 4 The Monte Carlo simulation 


\subsection{Introduction}

The birth of the Monte Carlo (MC) method for particle transport can be traced back to World War II where it was used in part of the Manhattan project to develop a thermonuclear weapon. The purpose was to use a statistical approach for solving the neutron diffusion problem and multiplication problems in fusion devices. There is no consensus on who first used MC method for particle transport but it is well known that Metropolis and Ulam published the first unclassified paper on the Monte Carlo methods (Metropolis and Ulam, 1949). In addition, R. Wilson published the first paper on Monte Carlo methods that included electron transport (Wilson, 1951).

The name was suggested by Metropolis et al (1949), referring to an administrative area of the Principality of Monaco where the primary attractions are casinos that have games of chance (Metropolis and Ulam, 1949, Metropolis, 1987). Since that time, the MC method has been used widely, i.e. calculating mathematical constants, general statistical analysis, nuclear physics, thermodynamics, quantum mechanics and radiation or particle transport (Seco and Verhaegen, 2013).

The MC method is a numerical method based on random sampling to approximate solutions to mathematical equations or integrals. The method produces a probability distribution for the quantity being estimated and the uncertainty can be calculated from this distribution. A single event or case can be estimated or simulated a number of times and the probability of different outcomes is provided. MC methods use random numbers, which are generated using a computer program called random number generator $(\mathrm{RNG})$, to generate random points between 0 and 1 with a specific distribution that resembles the probability of occurrence of the event being measured (the likelihood of the event occurring) (Rogers and Bielajew, 1990). A reliable average value can be achieved by simulating a large number of events that are associated with a statistical uncertainty. Since the result is averaged, as the number of simulated events is increased by a factor of 4 , the statistical uncertainty is reduced by a factor of 2 (Seco and Verhaegen, 2013).

For example, the MC method can be used to approximate $\pi$ of a circle with a radius $0.5 \mathrm{~cm}$ inscribed within a square of side equals to 1 . By taking the ratio of the area of a circle to the area of a square then multiplying by the area of the square, the value of $\pi$ can be estimated. Figure 4.1 shows an approximation of $\pi$ using the MC method 

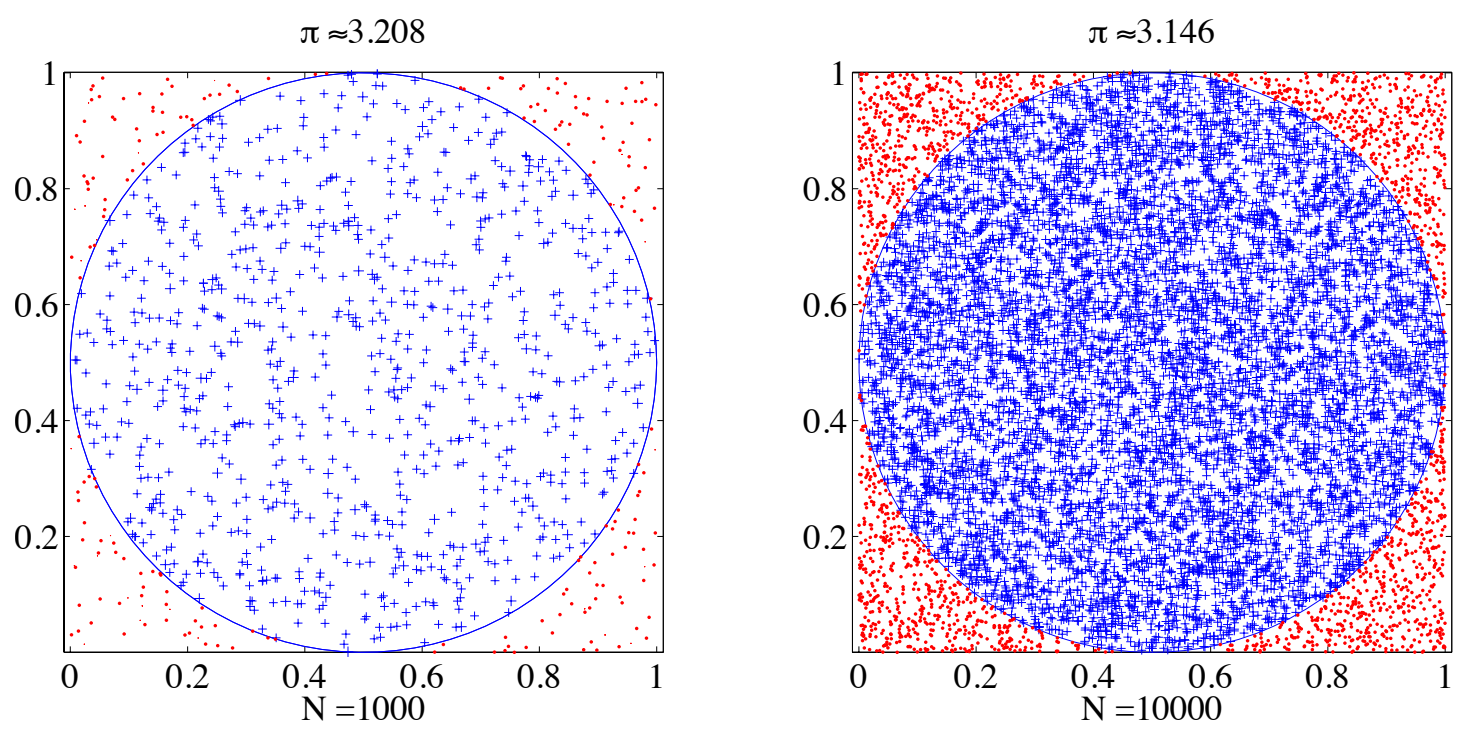

Figure 4.1: The estimation of the value of $\pi$ using the MC method. Left panel shows the estimated value of $\pi, 3.208$, using 1000 events. Right panel shows the estimated value of $\pi$, 3.146 , using 10000 events.

when two different numbers of events are used. It can be clearly seen that as the number of events or trials (N) increases from 1000 to 10000 the approximation is closer to the actual value and the statistical uncertainty is reduced from $7 \%$ to $0.1 \%$, respectively. Such a simple problem can be solved using deterministic or analytical methods but if the dimensionality of the problem becomes very large, the solutions of analytical or deterministic methods are impossible or computationally impractical whereas the MC method would be the most suitable method to solve the problem.

\subsection{Monte Carlo radiation transport}

The use of MC method in radiotherapy and dosimetry is to describe radiation transport by solving the Boltzmann transport equation (Duderstadt and Martin, 1979). Particle trajectories can be described by the linear Boltzmann transport equation:

$$
\left[\frac{\partial}{\partial s}+\frac{p}{|p|} \cdot \frac{\partial}{\partial x}+\mu(x, p)\right] \psi(x, p, s)=\int d x^{\prime} \int d p^{\prime} \mu\left(x, p, p^{\prime}\right) \psi\left(x^{\prime}, p^{\prime}, s\right)
$$

where $\mathrm{s}$ is the particle pathlength, $p$ is the momentum, $x$ is the particle position, $\mu(x, p)$ is the total macroscopic cross section and $\psi(x, p, s)$ is the probability of finding a particle in $\mathrm{d} x$ about $x$, in $\mathrm{d} p$ about $p$ and at $s$ (Seco and Verhaegen, 2013). 
The integral has multi-dimensional variables depending on the number of $x$ and $p$.

The Boltzmann transport equation is an equation of continuity in phase-space and applies to the microscopic physical laws that govern particle interactions, i.e. electronatom or/and photon-atom interactions. As the complexity of the problem increases (> 4D), such as in complex geometries, or if the dimensionality approaches infinity such as the calculation of dose distribution in patients, the analytical solution becomes impractical unless major approximations are applied. For example, the convolution method is used to estimate the number of particles reaching $x$ from phase-space located at $x^{\prime}, p^{\prime}$ with respect to $s$. In non-homogenous media or interfaces, the distance between $x^{\prime}$ and $x$ is scaled by the collision density, which describes the contribution from single scattered photons to the dose whilst approximating the multiple scatter contributions (see Chapter 3) (Seco and Verhaegen, 2013). The MC method can estimate the problem numerically and approach the true answer by introducing sampling errors according to the Central limit theorem (Feller, 1950, Seco and Verhaegen, 2013). Indubitably, the accuracy of the result depends on the accuracy of the geometry information provided, cross section data and the number of particle simulated. However, as the nature of radiation interaction with matter is probabilistic, the MC method is well suited.

There are many MC codes that have been used in radiotherapy modeling and dosimetry such as EGS, PENELOPE, FLUKA, MCNP and GEANT4. Each of these codes has particular advantages and limitations and, therefore, they have been used for different applications. In this study, EGSnrc, which is a version of EGS (Electron Gamma Shower) that was developed by the National Research Council (NRC; Canada), is used since it is mainly designed for modeling Linacs and is the most common code used for this purpose (see Chapter 5) (Kawrakow and Rogers, 2000).

\subsection{Photon transport}

\subsubsection{Photon interactions}

The microscopic physical laws that govern particle interactions are well known and governed by quantum electrodynamics. The probabilities of these interactions need to be included in MC codes to simulate particle transport accurately and are given by 


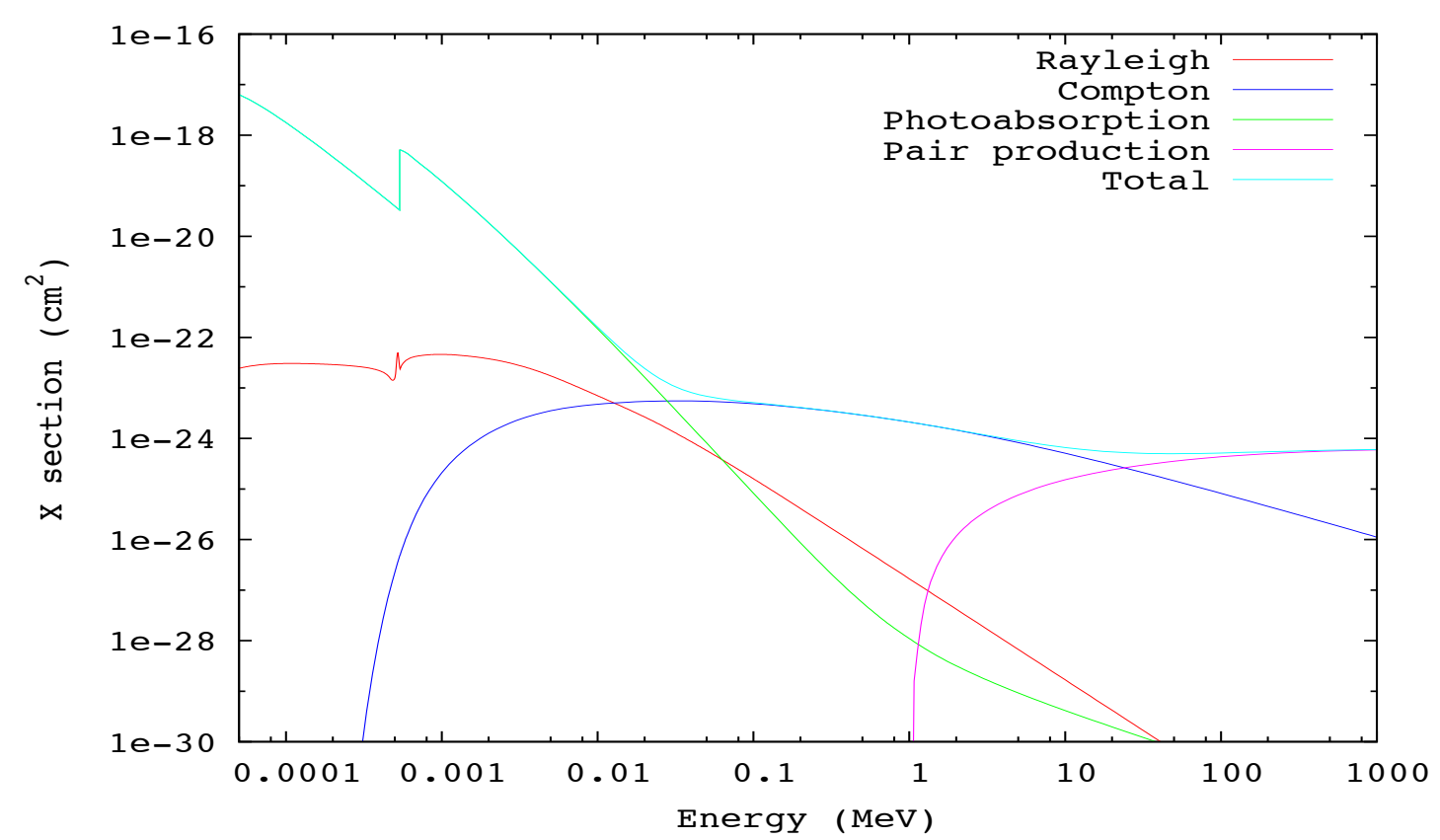

Figure 4.2: The cross-sections of different photon interactions $\left(\mathrm{cm}^{2}\right)$ against energy $(\mathrm{MeV})$ for water (generated using the PENELOPE MC code).

their cross sections. In radiotherapy, the possible photon interaction processes that should be modeled are:

1. Rayleigh scattering;

2. Compton scattering;

3. Photoelectric effect; and

4. Pair production.

Figure 4.2 shows the photon cross sections of the possible interactions that can occur in water over a range of energies. In general, the probabilities of these interactions occurring are dependent upon the energy of the incident photon and the atomic number of the absorbing material.

\subsubsection{Rayleigh scattering}

In Rayleigh scattering, a photon scatters elastically from bound electrons without transferring energy in a target atom, hence also called coherent scattering. Thus it involves only a change in direction of the photon. This interaction sets electrons into momentary vibration with the same frequency as the incident photon. Then these vibrating bound electrons emit electromagnetic radiation with the same energy as the 
incident photon. The scattered contributions will then combine, mainly in the forward direction. The cross section of Rayleigh scattering decreases with increasing incident photon energy and with decreasing atomic number. For high $\mathrm{Z}$ materials, it depends on $Z^{2}$ (Johns, 1983). Since no energy is transferred to the electrons, its cross section is usually forward peaked (particularly in low-Z) and since it is less than $10 \%$ of the total cross section in the therapeutic energies range, its contribution to energy deposition can be ignored, thus not important in radiation dosimetry.

\subsubsection{Compton scattering}

Unlike Rayleigh scattering, Compton scattering does not only involve a change in direction of the incident photon, but it also involves energy transfer to an electron, as kinetic energy, whose binding energy is a significantly small fraction of the incident photon, thus it is incoherent scattering. The Compton process ignores this binding energy and treats an electron as unbound or free. The maximum energy that can be transferred to an electron occurs when the incident photon is scattered with angle of $180^{\circ}$ of its original direction, and the electron is ejected, carrying out this energy in the forward direction with an angle of $0^{\circ}$. In this case the scattered photon will then carry out the minimum energy. The total energy is conserved in such a collision:

$$
h v=h v^{\prime}+E
$$

where $h v$ is the energy of the incident photon before the collision, $h v^{\prime}$ is the energy of the photon after the collision and $E$ is the energy of the recoil electron (Johns and Cunningham, 1983).

The scattering angle can vary from $0^{\circ}$ to $360^{\circ}$ thus the energy of the recoil electron can vary from 0 up to the possible maximum energy transferred which then will be either deposited or transferred to another electron within the medium via electronelectron interactions (see Section 4.5). As the energy of the incident photon increases, so does the probability of scattering in the forward direction and the fraction of the energy transferred per collision. For water (or tissue-like material), Compton scattering dominates at energies between $100 \mathrm{keV}$ and $10 \mathrm{MeV}$ as shown in Figure 4.2. Thus it is the dominant interaction in radiotherapy energies between 1 and 10 $\mathrm{MeV}$. For higher energy ( $>10 \mathrm{MeV}$ ), the probability of Compton scattering decreases 
linearly with increasing photon energy but it strongly dominates for low atomic number materials. Since there is energy transferred to electrons, it plays a major role in radiation dosimetry.

\subsubsection{Photoelectric effect}

One of the possible photon interactions that dominates at energies less than $100 \mathrm{keV}$ is the photoelectric effect. In this process, a photon interacts with a tightly bound electron, unlike Compton scattering where the electron is considered to be unbound. The photon is absorbed completely, giving up all its energy to the electron. Consequently, the electron, called photoelectron, leaves the atom with a kinetic energy that is equal to the difference between the incident photon energy and the electron binding energy, which dissipates in the absorbing medium mainly by excitation and ionisation. Thus the photoelectric effect does not occur when the incident photon energy is less than the electron binding energy. The atom is then left with a vacancy in one of its shells that is filled by one of the electrons from the outer shells, resulting in the emission of either fluorescence and/or Auger electron. The probability of the photoelectric effect occurring significantly increases when the incident photon energy is just slightly greater than the electron binding energy of each atomic shell of the absorption medium. The cross section curve exhibits discontinuities, referred to as absorption edges, which occur at energies corresponding to the binding energies of the atomic shells. The rest of the cross section curve varies as $1 / h v^{3}$ for low photon energies, where the photoelectric effect is dominant, and decreases linearly for high photon energies and increases as $Z^{n}$ where $n$ varies between 4 and 5 (Rogers and Bielajew, 1990).

\subsubsection{Pair production}

In pair production, a high energy photon interacts with the nuclear coulomb field. Similarly to photoelectric absorption, the incident photon is absorbed completely but in pair production an electron-positron, particle and anti-particle, pair is created following the conversion of energy into mass. Since the resting mass of an electron or positron is $m_{e} c^{2}$ ( $m_{e}$ is the rest mass and $c$ is the speed of light), the incident photon 


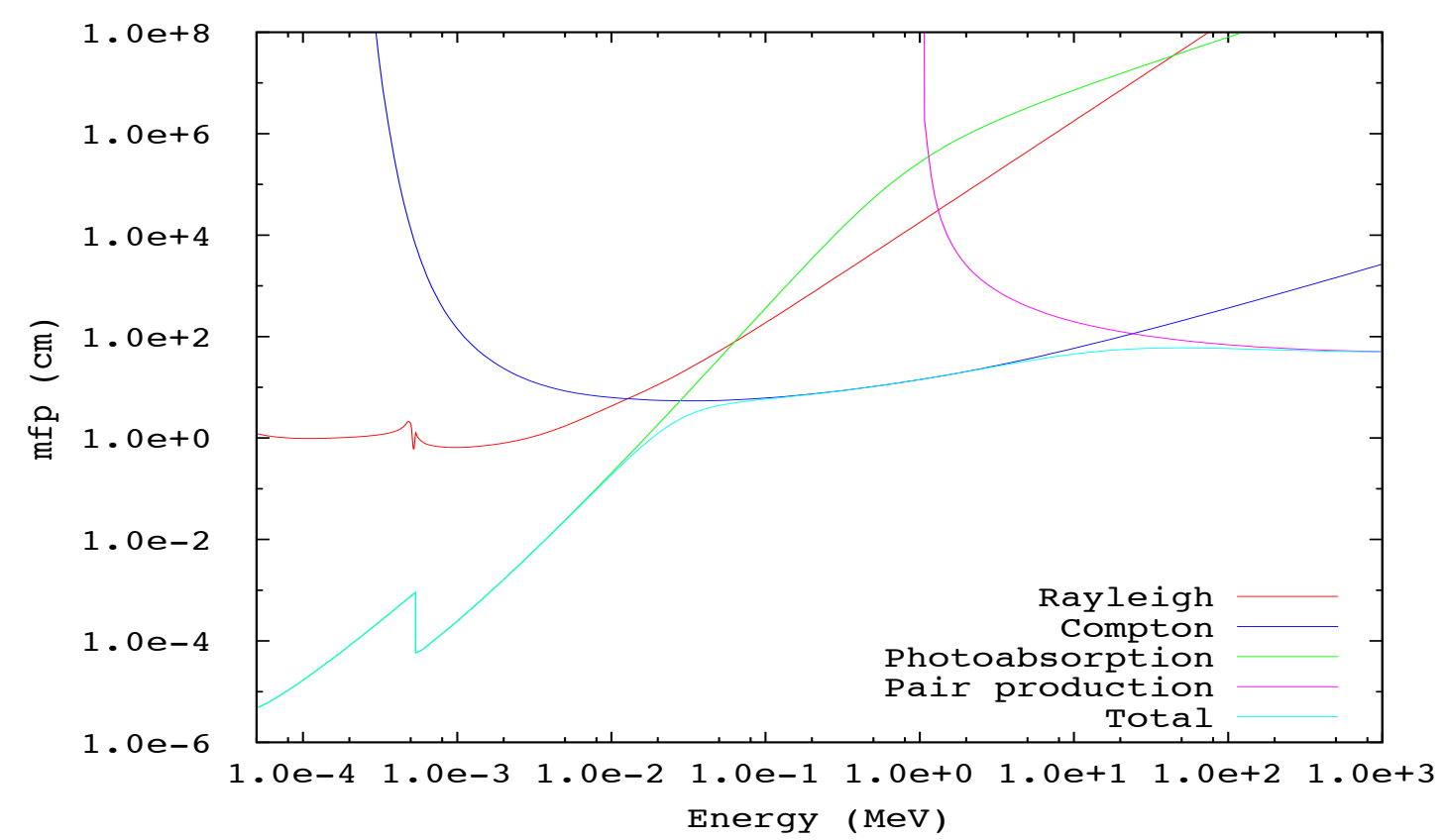

Figure 4.3: The photon mean free path to different photon interactions $(\mathrm{cm})$ against energy $(\mathrm{MeV})$ in water (generated using the PENELOPE MC code).

energy must exceed $2 m_{e} c^{2}$. If the incident photon energy is higher than this threshold, the additional energy is transferred into kinetic energy and is shared by the electron and the positron, which can be then deposited in the absorption medium. In this way, the energy and charge can be conserved. To conserve momentum a third object, usually a nucleus, is needed to absorb the additional momentum that is possessed by the incident photon, as the momentum before the interaction is always larger than the total momentum after the interaction mainly due to the fact that the electron-positron pair always have a velocity less than the speed of light. This additional momentum is extremely small, thus the nucleus gains a small amount of energy and this can be neglected compared with the electron-positron pair energy. The third object can be an orbital electron if the incident photon interacts with the atomic coulomb field, and will then be set in motion, hence the process is called triplet production. However, the positron travels through the medium and loses energy via excitation and ionization until it comes to rest then it annihilates with an electron, producing two photons with $0.511 \mathrm{MeV}$ opposite to each other in order to conserve momentum. In this way, the mass is converted back into energy. The probability of pair production occurring is low at photon energies comparable to the threshold and increases rapidly with increasing energy until it dominates when the photon energy approaches several $\mathrm{MeV}$ 
as shown in Figure 4.2. The cross section of pair production varies as $1 / Z^{2}$.

\subsubsection{Monte Carlo photon transport simulation}

In the therapeutic photon energy range, photons have fewer interactions compared with charged particles, electron or positron, as they pass through the patient. Some of these photons might not interact with the patient at all. The mean free path of a nominal $6 \mathrm{MV}$ beam (mean photon energy about $2 \mathrm{MeV}$ ) in water and human tissue is about $20 \mathrm{~cm}$ as shown in Figure 4.3. Thus, it would be expected that photons would interact only a few times in the patient, where the size of a region of interest is about $30 \mathrm{~cm}$ (Seco and Verhaegen, 2013). Primary photons lose their energies indirectly to the interacting medium as they produce energetic electrons via absorption or scattering, which in turn deposit energy along their paths. Consequently, these electrons can produce other electrons and photons.

In MC dose calculation, photon transport starts with defining the physical parameters such as location, momentum and energy, which is then used to sample from the appropriate cross section. Firstly, MC samples the mean free path length to the first interaction point, $s$, using the probability distribution given by exponential attenuation law and a uniform distributed random number $\xi_{1}$ :

$$
p(s)=e^{(-\mu s)}
$$

where $\mu$ is the linear attenuation coefficient of the interacting medium. The distance to the first interaction can be then obtained using:

$$
s=-\frac{\ln \left(\xi_{1}\right)}{\mu}
$$

In this way, the simulated photon can be traced through the simulated geometry taking into account different materials with different attenuation coefficients, which can be calculated using a database if the material compositions are known. EGSnrc MC code uses a variety of databases such as XCOM (Berger and Hubbell), EPDL (Cullen et al., 1990) and SI (Storm and Israel, 1970). For the geometry of the treatment head, data about the material compositions can be obtained from the manufacturer, whilst for the patient geometry it can only be obtained from CT 
numbers that are provided in their CT images. These CT numbers (or HU) are unique for each material. Thus mapping these CT numbers to specific human tissue types is required in order to calculate the attenuation coefficients in each voxel of the CT image. Using a small number of materials in the CT map or calibration is not recommended as the linear interpolation in the calibration can lead to enormous cross section uncertainties (Seco and Verhaegen, 2013). This shows how the cross sections have a strong dependence on the CT numbers and the calibration. For example, artefacts in the CT images produced by a high-density material can provide an inaccurate CT number and thus corrupt the cross section data and consequently decrease the dose calculation accuracy (see Chapter 8 and 9). However, after sampling the mean free path length to the first interaction site and calculating the interaction coefficients, the interaction type is then sampled using:

$$
\mu_{\text {total }}=\mu_{P E}+\mu_{C o h}+\mu_{C o m p}+\mu_{P P}
$$

where $\mu_{P E}, \mu_{C o h}, \mu_{C o m p}, \mu_{P P}$ are the linear attenuation coefficients for photoelectric absorption, coherent or Rayleigh scatter, Compton scatter and pair production, respectively. This involves the use of a second random number $\xi_{2}$. If the value of $\xi_{2}$ is less than $\mu_{P E}$, the interaction is then photoelectric absorption. For coherent scatter interaction, $\xi_{2}$ must be more than $\mu_{P E}$ and less than $\mu_{C o h}$, the same is applied for the other interactions. Then secondary particles are created by the chosen interaction, except for coherent scatter, and a third random number is used to sample energy and direction using the corresponding differential cross sections for that interaction. The secondary particles could be then simulated in the same manner as the primary photon starting with sampling the mean free path to the next interaction site, called analog particle transport. These secondary particles can produce more additional particles, which can be simulated in this analog manner. The path of a single primary particle, all of the secondary particles it generates, and all its products, is called a particle history. For dose calculation, an extra step is needed where the absorbed energy in each voxel is accumulated. At the end, the particle history is terminated if either the simulated particle leaves the simulated geometry or its energy drops below predefined minimum energies, called cut-off energies. Then another particle history is picked and the same analog manner is applied, and so on. 


\subsection{Electron transport}

\subsubsection{Electron interactions}

Since electrons are charged particles their interactions with matter are basically different from that of neutral particles, photons. As electrons travel through matter, they experience large numbers of small interactions and lose energy mainly in Coulomb interactions with the nuclei and bound atomic electrons of the interacting medium. In contrast to a photon where all its kinetic energy is dissipated with few interactions, an electron may undergo approximately $10^{5}$ individual interaction to lose all its kinetic energy depending on the initial energy (Attix, 2008). Due to the relativistic effects, the interaction can result in quite large changes in direction and energy. For example, the mass of a $2 \mathrm{MeV}$ electron is about 4.9 times the electron rest mass. Thus, as the energy of the incident electron decreases the cross section of an interaction occurring increases and the possibility of large deviations in the electron path increases as well. The electron creates a trail of ionisations and excitations along its path, and hence energy is deposited in the medium. Electron scattering affects the absorbed dose distribution in a medium, especially at media interfaces with different material densities (Andreo, 1985). A lateral displacement of the electrons can be caused by the angular deflections, thus widening the dose distribution. Therefore, the difference in scattering causes a perturbation in the dose distribution. However, in radiotherapy, the possible electron interaction processes that should be modeled in a MC code are:

\section{Elastic scattering}

2. Inelastic scattering and

3. Bremsstrahlung.

These interactions are shown in Figure 4.4. It shows the electron cross-section of different electron interactions against energy.

\subsubsection{Elastic scattering}

In elastic scattering, the incident electron interacts with one of the bound electrons of the interacting medium without energy transfer, only a change of direction. The 


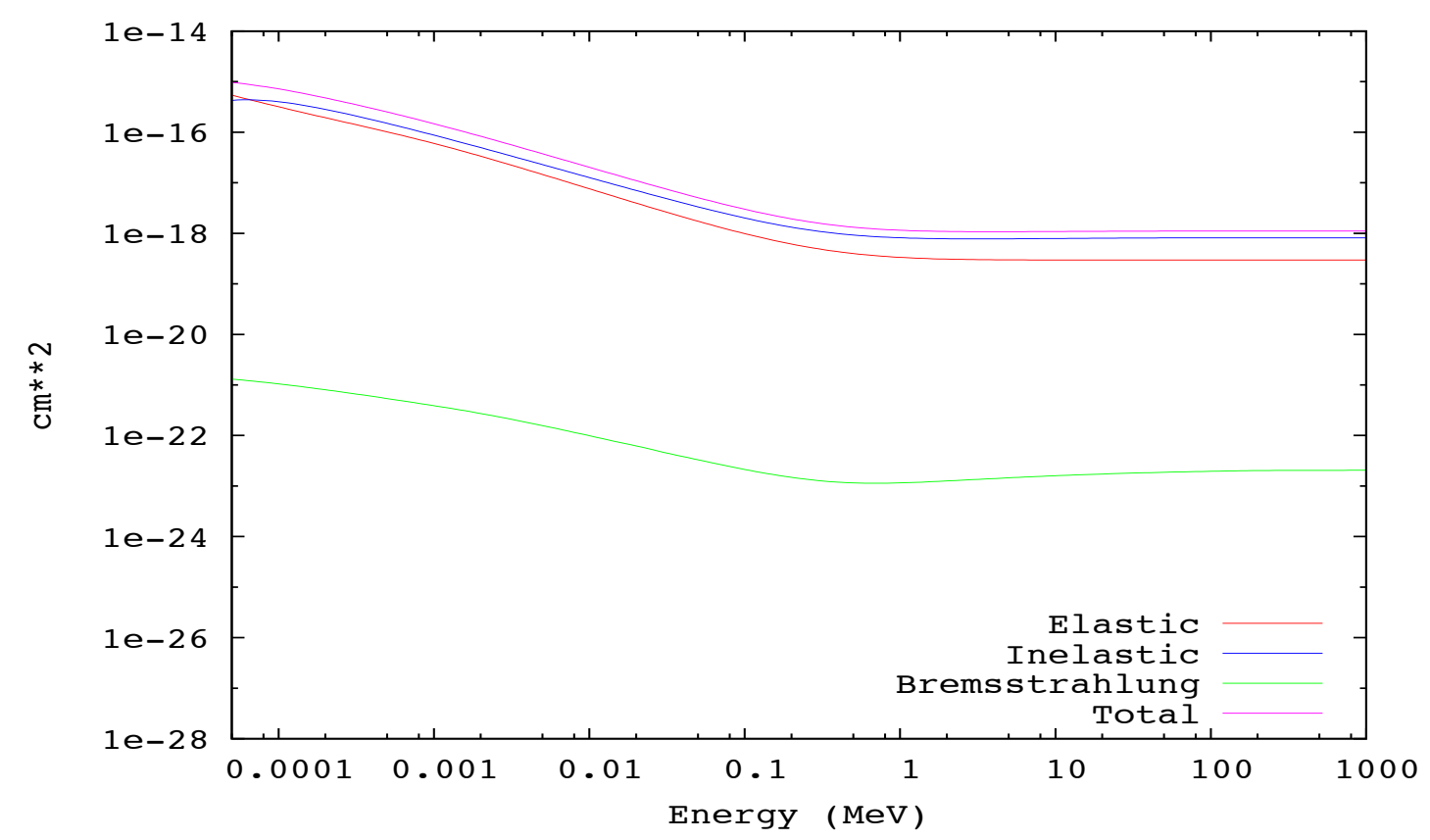

Figure 4.4: The cross-sections of different electron interactions $\left(\mathrm{cm}^{2}\right)$ against energy $(\mathrm{MeV})$ for water (generated using the PENELOPE MC code).

interaction is Coulomb interaction between the two negative charges and is modified at a large distance by the screening effect of the orbital electron (Jackson John, 1999). Such scattering does not contribute energy to the medium, but is an important mechanism in deflecting electrons. When a fast electron passes close enough to the atomic nucleus, at a distance smaller than the atomic radius, the Coulomb interaction takes place between the electron charge and the nuclear charge and is a repulsive interaction (Johns, 1983). In this case, the electron is deflected and a small amount of energy is transferred to the nucleus to satisfy momentum conservation. This energy transfer is considered to be negligible as the mass of the nucleus is much heavier than the relativistic mass of the incoming electron (Mayles, 2007). At energies lower than $10 \mathrm{MeV}$, elastic or semi-elastic scattering is the main energy loss mechanism.

\subsubsection{Inelastic scattering}

As mentioned before, due to the relativistically corrected mass of the incident electron, the interactions with orbital electrons of the interacting medium can result in a large energy loss and cause appreciable changes of direction, thus called inelastic scattering. If the incident electron passes an atom at a distance larger than the atom radius, and the energy it pocesses is less than the binding energy, this energy can be 
transferred to one of the orbital electrons, elevating the electron to a higher energy state. Thus the atom is excited. If the incident electron passes close enough to the orbital electrons, sufficient energy can be transferred to the orbital electron causing it to be ejected from the atom, thus the atom is ionised. The ejected electron can then also have enough energy to ionise and excite other atoms, just as the incident electron does. This is called $\delta$-ray (delta ray) and the event is called Møller scattering, where the maximum energy loss is half of the kinetic energy of the incident particle (Møller, 1932). At energies higher than $10 \mathrm{MeV}$, inelastic scattering represents the main energy loss mechanism, also called collision energy loss (Knoll, 2010). If the incident charged particle is a positron, the interaction with an orbital electron results in the emission of two photons. The cross section of this process is higher for positrons with a lower rest mass energy. On the other hand, at higher energies, a positron loses energy via collisions with the orbital electrons until it comes to rest. The same as in the case of an electron, a $\delta$-ray is produced and the event is called Bhabha scattering, where the maximum energy loss is the total kinetic energy of the positron (Bhabha, 1936). The cross section of this interaction is needed and should therefore be included in MC code that is used to calculate energy deposition.

\subsubsection{Bremsstrahlung production}

When a fast electron of a mass $m$ passes very close to a nucleus of a charge $Z$, it will undergo inelastic scattering with the nucleus. Due to the strong electric field of the nucleus, the electron will be attracted toward the nucleus, thus decelerated, and the electric and magnetic fields associated with the electron will adjust themselves to such a change. As a result, the electron emits some of its energy in the form of electromagnetic radiation called bremsstrahlung radiation, with an energy equal to the energy difference before and after the deceleration (Jackson John, 1999). The energy of the emitted photon can be up to the initial energy of the incident electron. The electron may have more than one bremsstrahlung interaction. In radiotherapy machine generating energies, only $\sim 3 \%$ of the incident electron energy emerges as bremsstrahlung radiation in a typical Tungsten target. The cross section of a bremsstrahlung interaction is proportional to $(Z / m)^{2}$, thus it increases directly with the atomic number of the interacting medium and with light particles such as electrons. Thus bremsstrahlung interactions are unimportant and insignificant in low atomic 
number materials at electron energies below $10 \mathrm{MeV}$, i.e. in water/tissues as shown in Figure 4.4. Bremsstrahlung radiation can also be produced if the incident particle is a positron, as well as positron-electron and electron-electron interactions. At energies higher than $10 \mathrm{MeV}$, together with the inelastic scattering, bremsstrahlung interactions represent the main energy loss mechanism, called radiative energy loss.

\subsubsection{Monte Carlo electron transport simulation}

The transport of electrons (or positrons) is fundamentally different from that of photons. It can be stated that the magnitude of the energy deposition problem for an electron beam is less than for a photon beam. This is due to the fact that the volume involved with the electron beam is smaller with a higher density of particle histories and the energy deposited per particle is higher (Rogers and Bielajew, 1990). Thus the variation from one history to another is reduced. As mentioned before, for therapeutic energies, electrons undergo a large number of single interactions and lose their energy in a near continuous set of interactions. These interactions include all the interactions mentioned in the previous section. Thus the MC simulation of a single electron history requires a larger computational time than a single photon history. This great increase in computational complexity for electron transport makes the analog approach (used in the photon case) and the direct simulation of all the physical interactions on an event-by-event basis unrealistic for practical use.

For example, the total mean free path in water for a $10 \mathrm{MeV}$ electron is about $2.6 \times$ $10^{-5} \mathrm{~g} / \mathrm{cm}^{2}$ and the range is approximately $5.03 \mathrm{~g} / \mathrm{cm}^{2}$ (as shown in Figure 4.5). This means that each electron with this energy will undergo about $2 \times 10^{5}$ interactions before slowing down. Fortunately, elastic scattering dominates the total cross section at this energy range (the therapeutic energy range), thus there is no energy transfer to the medium, although inelastic scattering still occurs but with little energy loss (Seco and Verhaegen, 2013). Thus as the electrons slow down in the medium, most of their interactions cause a small amount of energy loss or angular deflection. This allows the possibility of combining the effect of many elastic interactions and low energy-loss inelastic scattering within a certain length of geometry into one virtual large effect interaction (called step or segment) without significant loss of simulation accuracy. This large combined step is approximated using a technique based on continuous 


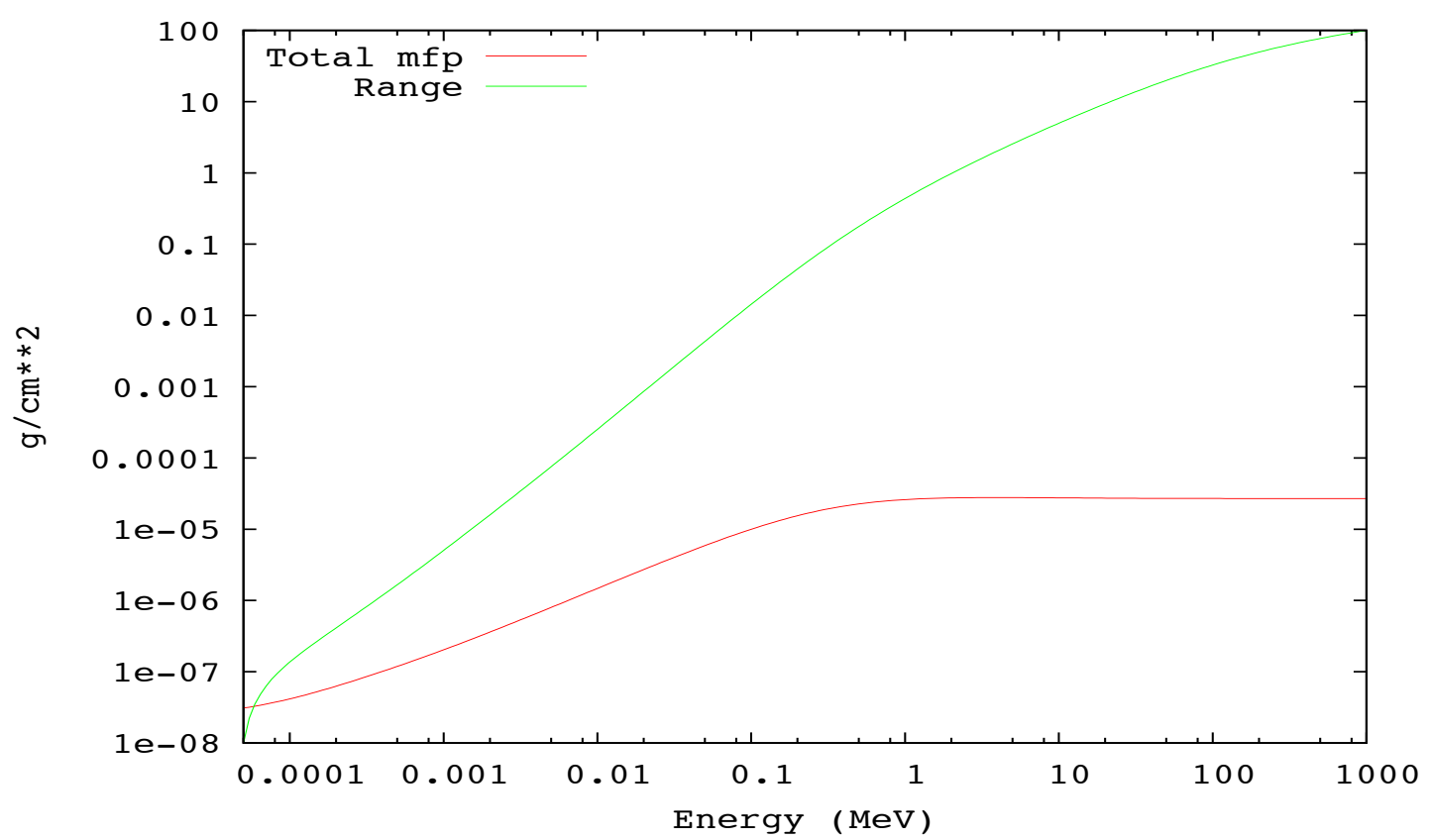

Figure 4.5: The electron mean free path and range $\left(\mathrm{g} / \mathrm{cm}^{2}\right)$ against energy $(\mathrm{MeV})$ in water (generated using the PENELOPE MC code).

slowing down approximation (CSDA) and a multiple-scattering theory (Bielajew et al., 1988, Seco and Verhaegen, 2013). Thus the MC codes usually employ this combining technique to speed up the calculation time and is called a condensed history $(\mathrm{CH})$ technique (Berger, 1963). As a consequence, the electron track is divided in multiple substeps. The $\mathrm{CH}$ technique is employed in two algorithm classes, class I and class II algorithm.

In class I algorithm, all energy losses and angular deflections of a certain type of interaction are grouped together for each $\mathrm{CH}$ step using CSDA. During the $\mathrm{CH}$ step, the effect of secondary particle creation above a certain threshold energy is taken into account on a statistical basis by sampling from an energy loss straggling algorithm (Bielajew et al., 1988). Then the energy of the primary electron is reduced by that amount at the end of the step. Thus the creation of the secondary electron does not directly affect the primary electron during the step. For example, MCNP (old versions) and ETRAN codes are class I algorithms.

In class II, the interactions are divided into "soft" and "hard" interactions or collisions. Electron interactions that release energy below a certain threshold are considered to be soft collisions and are grouped together in a single multiple scattering step using CSDA and restricted stopping powers. The energy is then 
deposited at the end of the $\mathrm{CH}$ step. On the other hand, above that threshold, the collisions are considered to be hard and receive explicit simulation and transport. EGSnrc is a class II algorithm as well as PENELOPE, GEANT4 and FLUKA codes. In general, electrons in $\mathrm{CH}$ step travel in straight lines and change direction due to only multiple scattering at the end step or between steps.

However, one of the most challenging aspect of $\mathrm{CH}$ technique is the simulation of an electron crossing material boundaries. If the $\mathrm{CH}$ technique is based on Molière multiple scattering theory, it fails to simulate the transport accurately because the ensemble of paths occurring in the adjacent medium cannot be taken into account. Thus there are new multiple scattering theories that switch to a single scattering model for short steps near material boundaries and then switch back to a multiple scattering model at larger steps in a uniform medium. In EGSnrc, Kawrakow et al (2011) developed such an algorithm called Exact that allows a simulated electron that reaches a boundary (defined by the user) to cross in a single scatter mode, shortening the step, then as the electron moves away from the boundary the step is lengthened again. It has been shown that Exact provides an accurate result even for large scattering angles (Seco and Verhaegen, 2013).

Briefly, the $\mathrm{MC}$ electron transport during $\mathrm{CH}$ step starts with picking the initial energy, direction and position of the particle stack. The path length (s) of a particle with initial energy $E_{0}$ is defined as

$$
s=\int_{0}^{E_{0}} \frac{d E^{\prime}}{S(E)}
$$

where $S$ is the unrestricted stopping power. This is the CSDA solution to the electron fluence transport. Based on the path length, sampling the distance to next discrete event can be done using a random number $(r)$ between 0 and 1 :

$$
\int_{0}^{s} d s^{\prime} \sigma^{t o t}\left(s^{\prime}\right)=-\ln r
$$

where $\sigma^{\text {tot }}$ is the total cross section. Then the energy of the next interaction that will be simulated explicitly is sampled. Then the final energy, direction and position are defined based on solving the electron transport equation, which is a function of restricted stopping power, differential and total cross sections. Then the type of 
interaction is sampled, if the electron is absorbed, the history is terminated, otherwise the energy, direction and position of the final state electron is defined based on the interaction differential cross sections. The end of the $\mathrm{CH}$ step can be terminated either by the maximum step size (defined by the user) or by the next hard interaction. If the next hard interaction is bremsstrahlung interaction, the resultant photon is then simulated using the analog technique.

Even though the $\mathrm{CH}$ technique speeds up the $\mathrm{MC}$ electron transport significantly compared to the analog technique, the efficiency of the MC simulation still needs to be improved for the calculation of the quantities of interest. Thus MC codes usually make use of variance reduction techniques (VRT) (Bielajew et al., 1988, Rogers et al., 1995). The efficiency of a MC simulation, $\varepsilon$, is defined as

$$
\varepsilon=\frac{1}{s^{2} T}
$$

where $s$ is the variance or uncertainty associated with the calculated quantity of interest and $T$ is the central processing unit (CPU) time required to calculate that quantity with an uncertainty $s$. The aim of a VRT is to minimize either $T$ or the uncertainty $s$ without affecting the accuracy of the calculation. Some of these VRTs are used in this study and will be explained in more detail in the appropriate sections.

\subsection{EGSnrc Monte Carlo code}

The most commonly used Monte Carlo code in medical physics, particularly in radiotherapy, is the Electron Gamma Shower code that was developed at the National Research Council in Ottawa, EGSnrc (Kawrakow and Rogers, 2000). The code allows the user to model a linear accelerator head precisely using a friendly graphical user interface (GUI), written in MORTRAN language, and simulates the physical photon and electron/positron processes in the energy range of $1 \mathrm{keV}$ to $10 \mathrm{GeV}$. It has been benchmarked against measurements and used as a reference for the validation of MC code when used for multiple purposes in the medical physics field (Chibani and Li, 2002, Faddegon et al., 2009). EGSnrc code consists of three main components. The first component is called user code which is used to specify the geometry (including phantoms), radiation source and output variables. Examples of EGSnrc user codes are 
BEAMnrc, DOSXYZnrc, DOSRZnrc and FLURZnrc. The second component is called EGS code where the electron and photon transports are simulated through the specified geometries. The last component is called PEGS4, which calculates the interaction cross-sections for the materials used in the simulation.

In this study, only two user codes are used, BEAMnrc and DOSXYZnrc codes. The BEAMnrc/DOSXYZnrc codes are used to simulate the beam generated from the treatment head and to score dose deposition in voxel grids of a $3 \mathrm{D}$ rectilinear geometry, respectively (Rogers et al., 2001, Walters et al., 2005). In general, BEAMnrc can be considered as the fluence engine whilst DOSXYZnrc can be considered as the dose engine. DOSXYZnrc can score dose deposition using real patient geometry obtained from a CT scan. This can be achieved by using a code called CTcreate or dicomrt_ctcreate (see Seection 6.2.6). It converts the CT number associated with each voxel in the CT images into material and density information needed by DOSXYZnrc to simulate transport in a patient using a calibration curve in MC database. Then, the interaction cross-sections can be calculated for each voxel using the assigned material information, such as density and composition. Thus the cross sections have a strong dependence on the CT numbers, which can decrease the dose calculation accuracy if artefacts present.

In the next chapter, the specifics of MC modeling with EGSnrc (BEAMnrc and DOSXYZnrc codes) system will be discussed and the code system is validated with the measurements. The validated model will be then used to benchmark for comparison with OMP (PB and CC algorithms) treatment plans. Furthermore, it will be used for cases where dose calculation is known to be particularly uncertain such as for a prostate cancer patient with single hip or double hip prostheses. 
Chapter 5 Monte Carlo modelling of the linear accelerator head 


\subsection{Introduction}

For radiotherapy purposes, a linear accelerator (Linac) is made of several components that are used to shape the radiation field. In MC, a Linac can be easily modelled using BEAMnrc GUI, which is considered to be a major step forward in the radiotherapy field (Seco and Verhaegen, 2013). It allows the user to build the Linac in such a way that the treatment head is divided into blocks, layers or slabs, called component modules. An example of component modules (CM) is the MLCQ CM, designed to model the MLCs, or the FLATFELT CM, designed to simulate a stack of truncated cones, each with different radius values and material definitions, which are used to model the flattening filter. To build an accurate MC model, the different components of the Linac through which the emitted radiation passes must be known in great detail. This information includes the position, dimension, composition of materials and alloys, and the mass densities. In this chapter, the modelling of the Elekta Synergy linear accelerator head for a $6 \mathrm{MV}$ photon beam only is presented.

The Linac was modelled based on manufacturer information, including aspects developed by previous PhD students (Piliero, 2014, Alsaleh, 2014). However, there are usually differences between the simulation and measurements. This is because there is no specific information about the exact energy or radial distribution of the electrons that are producing the bremsstrahlung photons from the target. The electron energy is the main source affecting the percentage depth dose (PDD) curve, whilst the radial distribution (spot size) affects the lateral dose profiles, especially for large field sizes (Lin et al., 2001, Verhaegen and Seuntjens, 2003). Thus the aim of this chapter is to validate or tune the beam model of the simulation, which is reached through gaining agreement between simulation and measurement.

\subsection{Materials and methods}

\subsubsection{Tuning and validation of the Linac model}

The Elekta Synergy linear accelerator head at Singleton Hospital, which contains; Target, primary collimator, flattening filter, chamber, backscatter plate, multi-leaf collimator (MLC), backup jaws, secondary collimator and Mylar sheet CM, was modelled using BEAMnrc code, as shown in Figure 5.1. The material of each CM is 


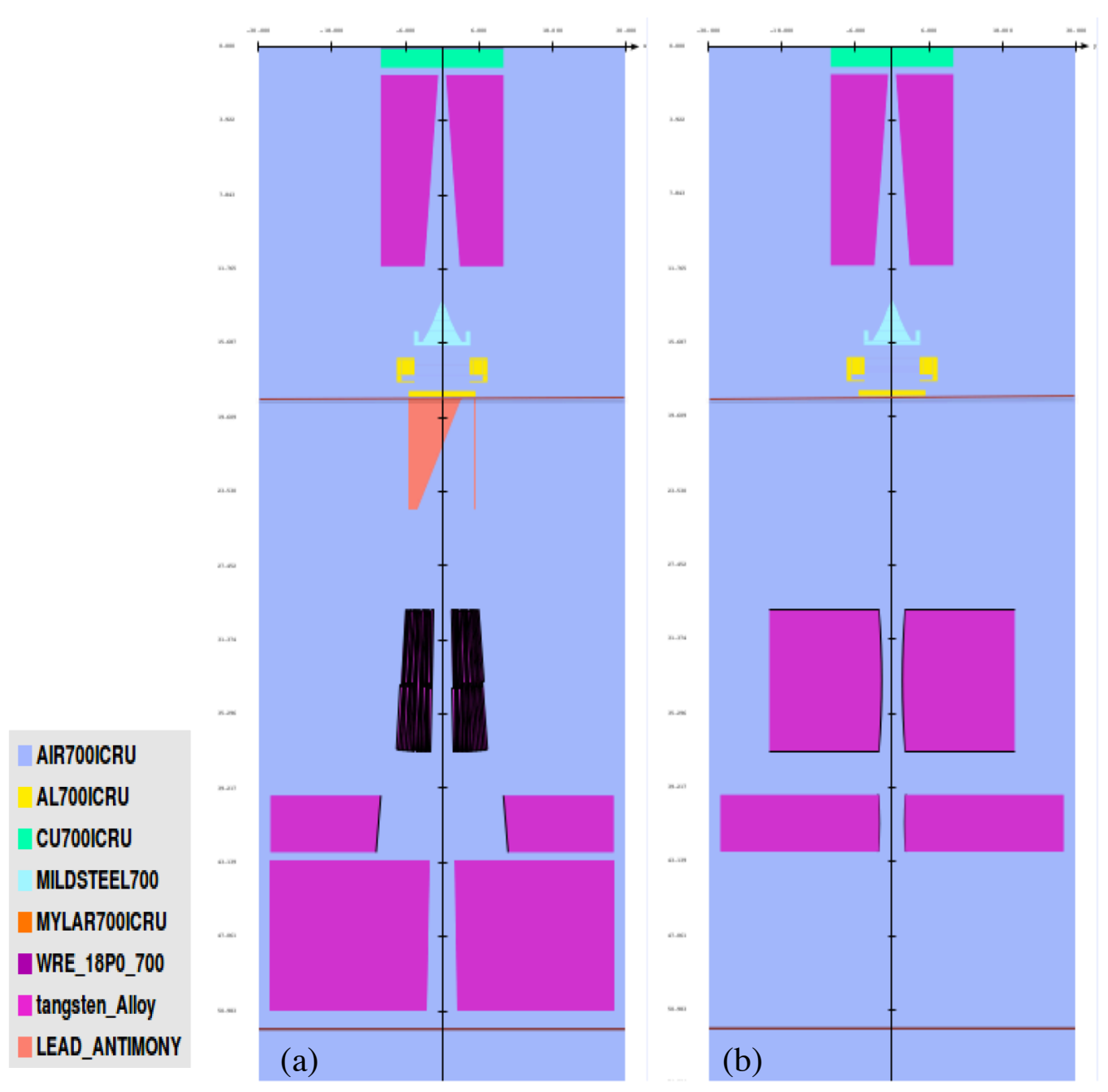

Figure 5.1: A view of the Linac $6 \mathrm{MV}$ model in (a) the $x z$ and (b) the yz plane.

shown in Table 5.1. The beam intensity and the size of the beam field are defined using adjustable components such as MLC, backup jaws and secondary collimator, whilst the rest of the components are fixed. In this chapter, the phase-space approach of simulating a Linac and patient is used, which involves separating the simulation into three distinct phases (Verhaegen and Seuntjens, 2003). In the first phase, source number 19 (elliptical beam with Gaussian distribution in X and Y) in BEAMnrc was used to model the incident electrons on the target. Then all the simulated particles that pass through the fixed components of the head, and reach an arbitrary plane perpendicular to the beam direction, are stored in a file called the phase space file (PHSP). The PHSP contains information such as particle energy, particle type, position and direction, and can be re-used without repeating the simulation for these fixed components since the simulation of a particle at any point is independent of its 
previous history (Rogers and Bielajew, 1990). This PHSP file is patient-independent and is used as an input source for the next phase of the simulation.

Table 5.1: The component models and materials of the 6 MV Linac model.

\begin{tabular}{|c|c|c|}
\hline CM & Material & CM description \\
\hline SLABS & $\begin{array}{c}\text { Tungsten (WRE_18P__700) } \\
\text { Copper (CU700ICRU) }\end{array}$ & Target \\
\hline CONS3R & Tungsten Alloy & Primary collimator \\
\hline FLATFILT & $\begin{array}{c}\text { Stain Steel } \\
\text { (MILDSTEEL700) }\end{array}$ & Chamber \\
\hline CHAMBER & $\begin{array}{c}\text { Aluminium } \\
\text { (AL700ICRU) }\end{array}$ & Backscatter plate \\
\hline SLABS & $\begin{array}{c}\text { Aluminium } \\
\text { (AL700ICRU) }\end{array}$ & Wedge \\
\hline JAWS & $\begin{array}{c}\text { Lead } \\
\text { (PB700ICRU) }\end{array}$ & Multileaf collimator \\
\hline MLCE & Tungsten Alloy & Backup jaws \\
\hline MLCQ & Tungsten Alloy & Secondary collimator \\
\hline JAWS & Tungsten Alloy & Mylar sheet \\
\hline SLABS & $\begin{array}{c}\text { Mylar } \\
\text { (MYLAR700ICRU) }\end{array}$ & \\
\hline
\end{tabular}

In the second phase, source number 21 in BEAMnrc (full phase-space beam data, incident on any $\mathrm{CM}$ ) was used to transport the particles from the PHSP file through the adjustable components such as jaws and multi-leaf collimators. The simulated particles are then stored in a second PHSP file that is placed below the adjustable components. This is a patient-dependent phase and the output is then used as an input source for the next step of the simulation. In the final phase, source number 2 in DOSXYZnrc (full phase-space source file) was used to transport particles from the second PHSP file into a phantom or patient, and the dose is scored. To provide an accurate simulation with a low statistical uncertainty, the PHSP file in the first phase should have a large number of particles. In this chapter, 50 million particles were used for each simulation to reduce the statistical uncertainty to less than $2 \%$ in the calculated dose at the maximum dose depth.

As mentioned before, any Linac model has to be validated with the commissioning measurement of the Linac, as there is some missing information, i.e. information on the exact energy and radial distribution of the electrons that produce bremsstrahlung 
photons from the target. This information can be predicted by performing a trial-anderror procedure to tune the Linac model with the commissioning measurement. The PDD of a $6 \mathrm{MV}$ photon beam (the absorbed dose in a medium as a function of depth along the beam direction) and the lateral dose profiles (the absorbed dose along $\mathrm{x}$ and $\mathrm{y}$ axes perpendicular to the beam direction at a certain depth in a medium) were measured in a PTW MP3 water tank (PTW, Freiburg, Germany), with a PTW semiflex ionisation chamber volume of $0.125 \mathrm{cc}$, using different square fields. For tuning the electron energy value, the PDD of the simulation, and the measurements of different field sizes $\left(3,5,7\right.$ and $\left.10 \mathrm{~cm}^{2}\right)$ at a source-to-surface distance (SSD) of 90 $\mathrm{cm}$, were compared using different electron energies. Once the closet match was obtained, the lateral dose profiles of different field sizes $\left(5,10,15\right.$ and $\left.20 \mathrm{~cm}^{2}\right)$, at different depths $(1.5,5,10,20 \mathrm{~cm})$, were compared using different spot sizes with and without a wedge. For all field sizes, dose profiles with a $90 \mathrm{~cm} \mathrm{SSD} \mathrm{were} \mathrm{normalised}$ to the central axis value of the dose profile at a depth of $1.5 \mathrm{~cm}$, which is the maximum depth dose for a $6 \mathrm{MV}$ beam. Only the best matches of both electron energy and spot size will be demonstrated in this chapter.

The combination of the electron beam and the spot size was used to calculate the output factor, and the quality index was then compared with the measurement. The output factor (OF) is defined as the ratio of the dose for a given field size to that of a reference field size, usually $10 \mathrm{~cm}^{2}$. The quality index (QI), or tissue-phantom ratio (TPR ${ }^{20} /{ }_{10}$ ), is the ion chamber value at a depth of $20 \mathrm{~cm}$, divided by the value at 10 $\mathrm{cm}$ for the same source-to-chamber distance (SCD), and with a field size of $10 \mathrm{~cm}^{2}$, thus with different SSDs.

\subsubsection{Simulation parameters}

Most of the simulation parameters used in the BEAMnrc and DOSXYZnrc codes were left at their recommended (default) values, as shown in Table 5.2. The energy cutoff for electrons and photons (ECUT and PCUT), and the energy of secondary electrons and bremsstrahlung production (AE and AP), were set to $700 \mathrm{keV}$ and 10 $\mathrm{keV}$ respectively. Below these energy thresholds, all the interactions were considered to be soft collisions and the particle histories were terminated, thus all the energy was deposited locally. Above these thresholds, all the interactions were considered to be 
hard collisions and were simulated explicitly in an analog manner (see Section 4.4.2). The maximum step size, which is based on the percentage energy loss during the step, was set to $25 \%$. This means that the step size depends on the mass density and stopping power of the interacting material.

Table 5.2: The transport parameters used in BEAMnrc and DOSXYZnrc in this study.

\begin{tabular}{|l|l|}
\hline Option & Parameter \\
\hline Bound Compton scattering & Off \\
\hline Pair angular sampling & Simple \\
\hline Pair cross section & BH (Bethe-Heitler) \\
\hline Photoelectron angular sampling & Off \\
\hline Rayleigh scattering & On \\
\hline Atomic relaxations & Off \\
\hline Photon cross-sections & si \\
\hline Boundary crossing algorithm & EXACT \\
\hline Electron-step algorithm & PRESTA-II \\
\hline Spin effect & On \\
\hline Electron impact ionisation & Off \\
\hline Bremsstrahlung angular sampling & Simple \\
\hline Bremsstrahlung cross-sections & BH \\
\hline
\end{tabular}

\subsubsection{Voxel water phantom}

DOSXYZnrc was used to model the watertank. The simulations were performed on a $41 \times 41 \times 70$ voxel water phantom. The particles were transported through the phantom on a voxel-by-voxel basis and the energy deposition was scored in each voxel. This was then converted into a dose deposition at the end of the simulation. For PDD curves, the voxel size was $1 \times 1 \times 0.75 \mathrm{~cm}^{3}$ in the build-up region and $0.5 \times 0.5$ $\times 0.5 \mathrm{~cm}^{3}$ from $2 \mathrm{~cm}$ onwards.

\subsubsection{Variance reduction techniques}

Even though the $\mathrm{CH}$ technique speeds up $\mathrm{MC}$ electron transport significantly compared to the analog technique, the efficiency of MC simulation still needs to be improved for the calculation of the quantities of interest. Thus MC codes usually make use of VRT (Bielajew et al., 1988, Rogers et al., 1995). The aim of a VRT is to minimize either calculation time or the uncertainty without affecting the accuracy of 
the calculation.

\subsubsection{Bremsstrahlung splitting}

One of the VRT used in this chapter is bremsstrahlung splitting in BEAMnrc (Rogers et al., 2001). When electrons hit the target, only $\sim 3 \%$ of the incident electron energy emerges as bremsstrahlung photons in a typical Tungsten target, and 97\% are converted into heat within the target. Thus for one million bremsstrahlung photons, at least 33 million histories are needed and this provides a low statistical accuracy as the variation from one history to another is large (see Section 4.4.2). Therefore a very large number of particle histories are needed and thus the simulation will be very long.

The Bremsstrahlung splitting technique is used to improve the statistics of bremsstrahlung photons whilst retaining a reasonable calculation time. When bremsstrahlung radiation is emitted, each bremsstrahlung event produces a predefined number of independent photons $(N)$ and each of them is assigned with a statistical weight equal to the inverse of $N$, called splitting number. Thus one bremsstrahlung photon is represented by $N$ number of simulated photons and the total weight is preserved. Therefore the transport simulation of $N$ additional electrons is avoided to increase the statistical accuracy and consequently reduces the simulation time. In this chapter directional bremsstrahlung splitting (DBS) is used. After splitting, DBS loops through these split photons and for each one determines whether it is aimed at the field of interest. If it is, then the photon is kept and given a low weight (meager photon), if not, the Russian Roulette technique (another VRT) is applied to determine its survival by comparing a random number, $(\zeta)$, sampled from a uniform distribution, with the survival probability $(1 / N)$. If $\zeta$ is larger than the survival probability, the photon is killed, otherwise the photon is simulated and its statistical weight is multiplied by $N$, and it is considered to be a fat photon. This photon can be split when it undergoes interactions (annihilation or Compton event) and if the newly created photon is aimed away from the field of interest, Russian Roulette is played again. In this way, many meager photons will be in the splitting field and few fat photons outside the splitting field. Therefore, terminating the photons outside the field of interest saves the simulation time. DBS is considered to be the most efficient 
bremsstrahlung splitting technique (Rogers et al., 2001). The DBS parameters were chosen as follows; the square splitting field radius was $30 \mathrm{~cm}$, the SSD was $100 \mathrm{~cm}$ and the splitting number was set to 500 using a rejection plane at $85 \mathrm{~cm}$.

\subsubsection{Photon splitting}

Another VRT used in this chapter is called photon splitting and it is used in DOSXYZnrc (Walters et al., 2005). When these split photons, with different weights in the Linac model, reach the voxel water phantom, each of them is splitting into a number of photons $M$. Each of these sub-photons will be assigned a weight which is the inverse of $M$ multiplied by the original photon weight. Along the initial photon path, the interaction site of each of the sub-photons is sampled. At the interaction site, the produced charged particles, which deposit energy along their paths, are kept whilst scattered photons undergo Russian Roulette with a survival threshold given as the inverse of $M$. In this way, the efficiency of the dose calculation in the phantom is increased.

\subsubsection{HOWFARLESS}

In DOXYZnrc, the HOWFARLESS option was used. Since the MC calculations were made in a water phantom, the voxel boundary restrictions were not necessary because it is a homogenous medium, thus they can be removed or eliminated during the simulation. Instead, only the outer boundaries of the voxel grid, the phantom boundaries, were considered. The separation of the voxel grid from the homogenous phantom geometry was done by HOWFARLESS option. In this way, the simulation of each history was not interrupted at each voxel boundary, thus the efficiency of the calculation is increased without loss of simulation accuracy.

\subsection{Result and discussion}

\subsubsection{PDD and dose profile comparison}

The measured PDD curves were obtained from Singleton Hospital, for $3 \times 3 \mathrm{~cm}^{2}, 5 \times$ $5 \mathrm{~cm}^{2}, 7 \times 7 \mathrm{~cm}^{2}$ and $10 \times 10 \mathrm{~cm}^{2}$ field sizes with a $90 \mathrm{~cm}$ SSD in a watertank. The simulated PDD curves were calculated on a voxel water phantom in DOSXYZnrc 

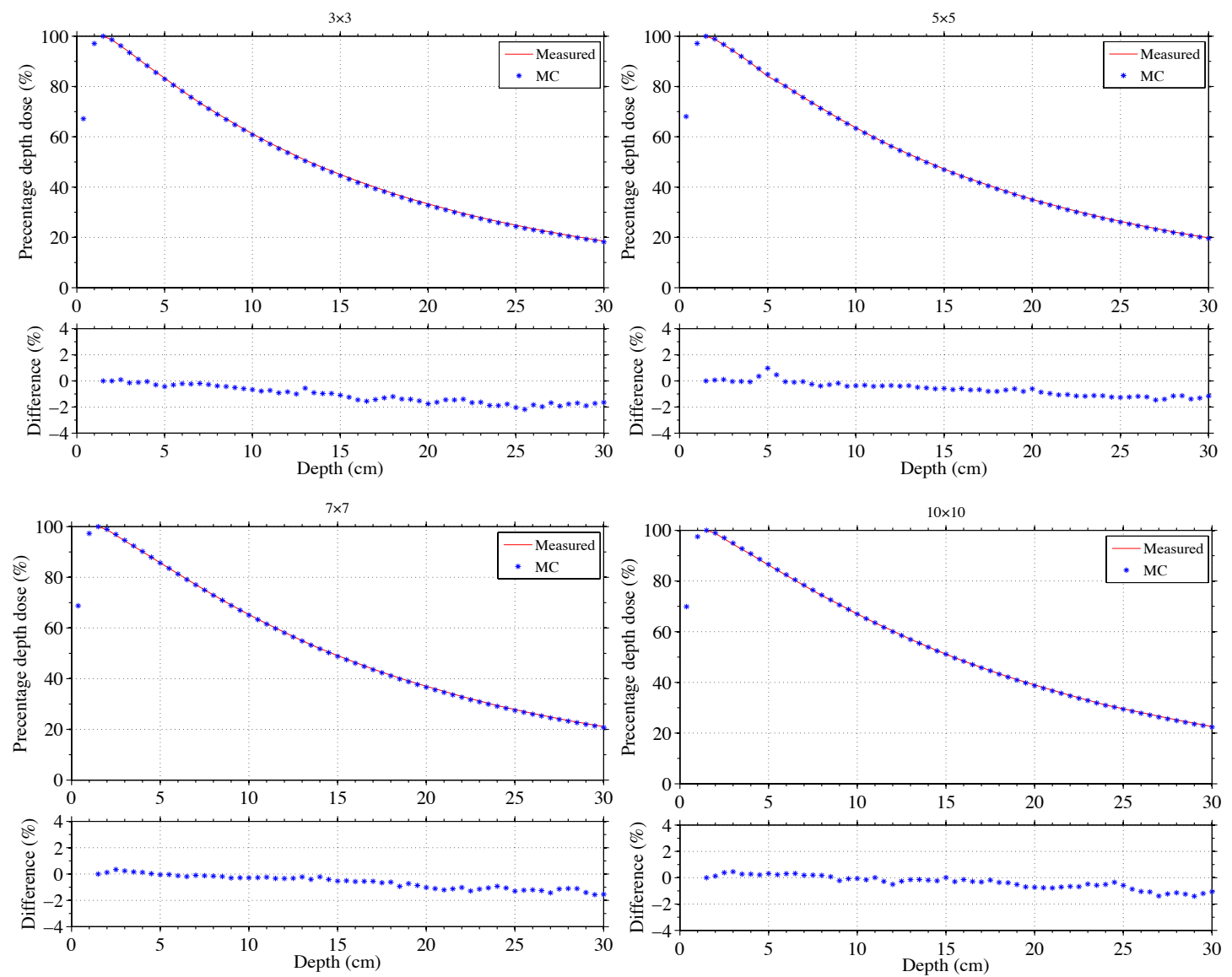

Figure 5.2: Comparison between measured PDD data of the 6 MV beam (red solid line) and simulated PDD for $3 \times 3,5 \times 5,7 \times 7$ and $10 \times 10 \mathrm{~cm}^{2}$ field size at $90 \mathrm{~cm}$ SSD with 6.05 $\mathrm{MeV}$.

using the same field sizes and settings. The energy of the electrons hitting the target, and producing the photon beam in the Linac model, was adjusted to find the best agreement with the measurement. After the tuning process, which included a considerable amount of trial-and-improvement, the best agreement between simulation and measurement was found when using a 6.05 MeV electron energy.

Figure 5.2 shows the PDD comparison between measurement and simulation for different field sizes using a $6.05 \mathrm{MeV}$ electron energy. For the $10 \times 10 \mathrm{~cm}^{2}$ field size PDD comparison, the relative difference was less than $\pm 1 \%$ before a depth of $25 \mathrm{~cm}$ and the largest relative difference was at a depth of $27 \mathrm{~cm}$ where the simulation underestimated the relative dose by $-1.5 \%$. For the $7 \times 7 \mathrm{~cm}^{2}$ and $5 \times 5 \mathrm{~cm}^{2}$ field sizes the relative dose was less than $\pm 1 \%$ and beyond a depth of $20 \mathrm{~cm}$ it increased up to $-1.8 \%$. For the $3 \times 3 \mathrm{~cm}^{2}$ field size PDD comparison, the largest relative difference 

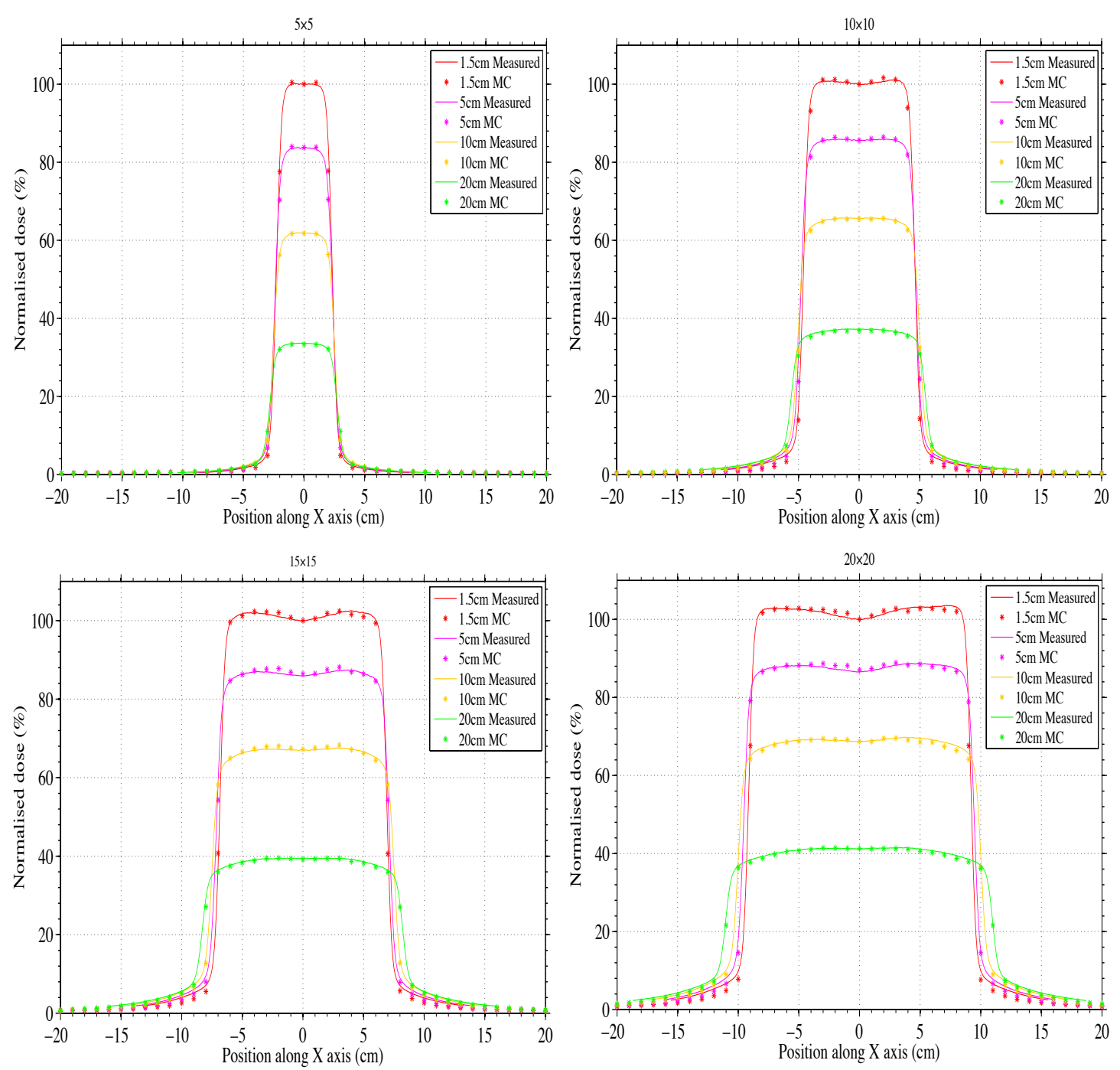

Figure 5.3: Lateral dose profiles comparisons for open beam between measured (solid line) and simulation (dotted line) of $5 \times 5,10 \times 10,15 \times 15$ and $20 \times 20 \mathrm{~cm}^{2}$ at 1.5, 5, 10 and 20 $\mathrm{cm}$ depths along jaws axis using $6.05 \mathrm{MeV}$ electron energy with $0.14 \mathrm{~cm}$ spot size.

was at a depth of $25.5 \mathrm{~cm}$ where the simulation underestimated the relative dose by $2.2 \%$. This is considered to be acceptable (Verhaegen and Seuntjens, 2003).

After finding the best electron energy match, the spot size was adjusted in order to obtain the best match between measurement and simulation in terms of lateral dose profile comparison. The best match was found when using an circular shape for the electron spot with a full-width half-maximum (FWHM) of $0.14 \mathrm{~cm}$. Figure 5.3 shows the lateral dose profile comparisons between measurement and simulation for $5 \times 5$ $\mathrm{cm}^{2}, 10 \times 10 \mathrm{~cm}^{2}, 15 \times 15 \mathrm{~cm}^{2}$ and $20 \times 20 \mathrm{~cm}^{2}$ at different depths using the best match spot size. For all field sizes, dose profiles with a $90 \mathrm{~cm}$ SSD were normalised to the central axis value of the dose profile at a depth of $1.5 \mathrm{~cm}$. The results showed 

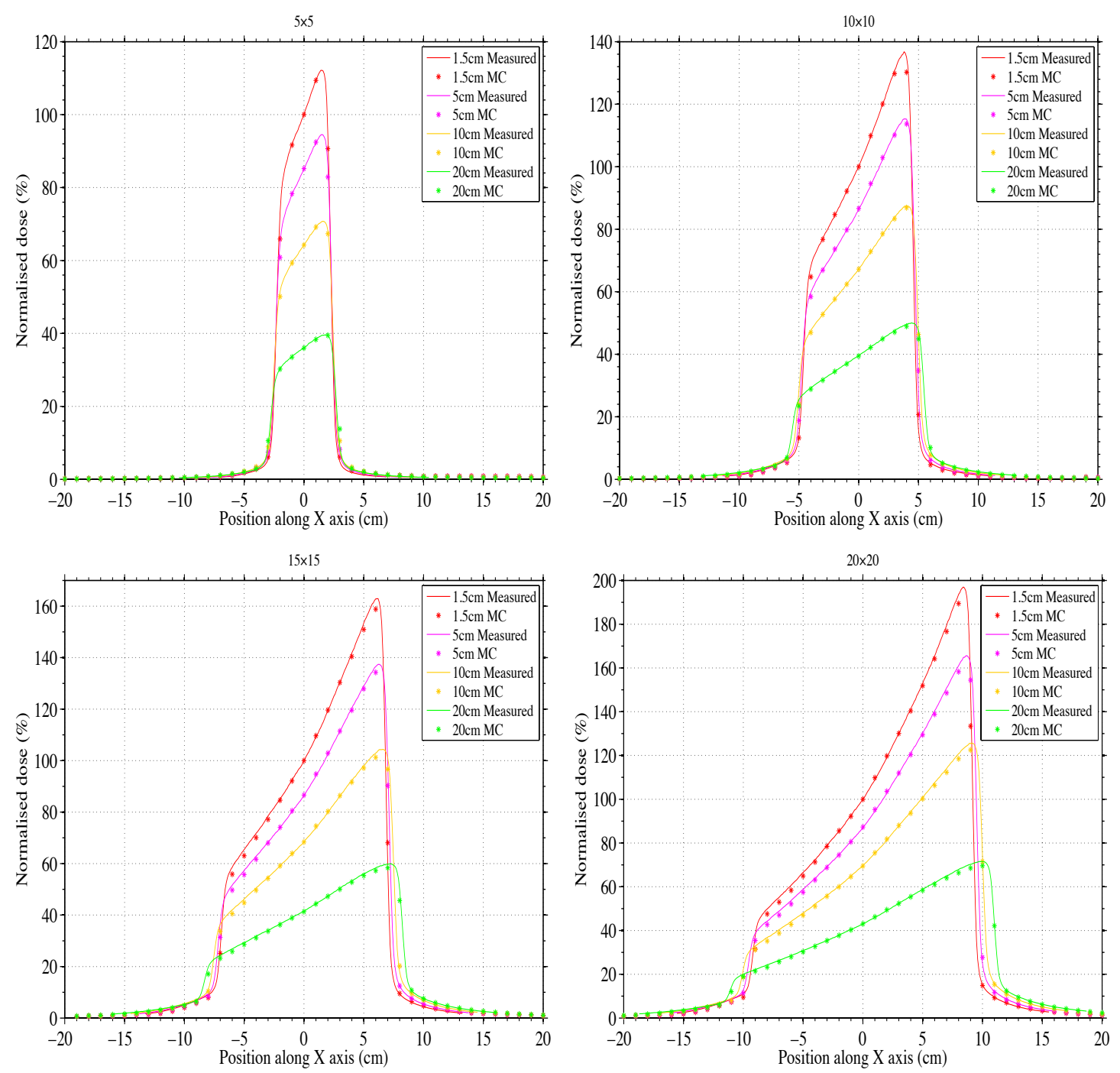

Figure 5.4: Lateral dose profiles comparisons for wedged beam between measured (solid line) and simulation (dotted line) of $5 \times 5,10 \times 10,15 \times 15$ and $20 \times 20 \mathrm{~cm}^{2}$ at 1.5, 5, 10 and $20 \mathrm{~cm}$ depths along jaws axis using $6.05 \mathrm{MeV}$ electron energy with $0.14 \mathrm{~cm}$ spot size.

that the difference between measurement and simulation for a $10 \times 10 \mathrm{~cm}^{2}$ field size, at any depth, was less than $\pm 1 \%$ inside the field and increased up to $\pm 3 \%$ as the filed size increased to $20 \times 20 \mathrm{~cm}^{2}$. In the low dose (below $20 \%$ of the normalized dose) region, the relative difference was $-13 \%$ (in $2 \mathrm{~mm}$ ) for a $10 \times 10 \mathrm{~cm}^{2}$ field size and increased up to $-18 \%$ which decreased as the depth increased. The largest disagreement was found outside the field in the low dose region.

Furthermore, the Linac model was compared with the measurement using a wedge for the same field sizes used in the open fields. Figure 5.4 shows the lateral dose profile comparisons between measurement and simulation for different field sizes, at different depths, using a wedge. The agreement between measurement and simulation 

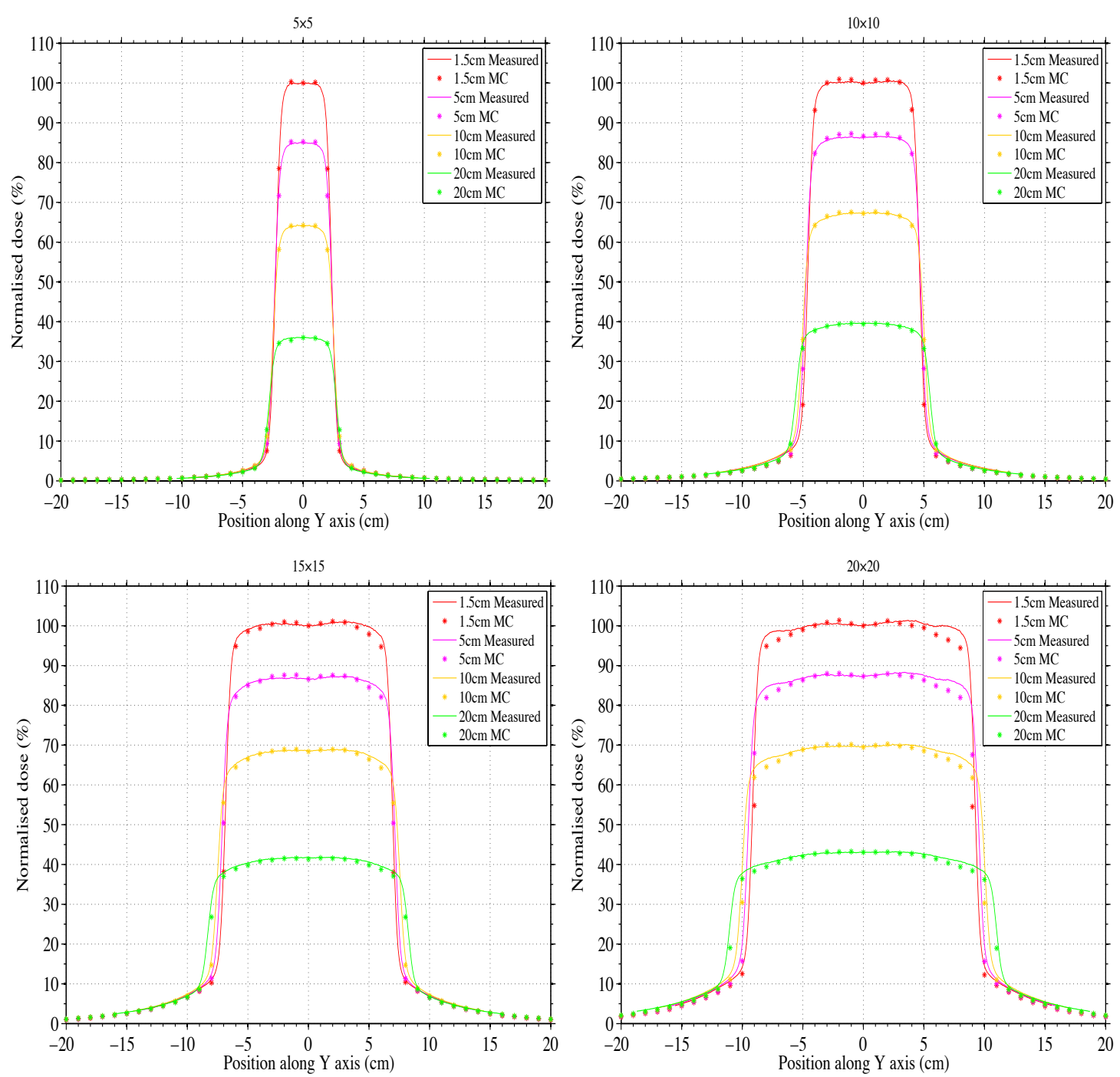

Figure 5.5: Lateral dose profiles comparisons for wedged beam between measured (solid line) and simulation (dotted line) of $5 \times 5,10 \times 10,15 \times 15$ and $20 \times 20 \mathrm{~cm}^{2}$ at 1.5, 5, 10 and $20 \mathrm{~cm}$ depths along MLC axis using $6.05 \mathrm{MeV}$ electron energy with $0.14 \mathrm{~cm}$ spot size.

for a $10 \times 10 \mathrm{~cm}^{2}$ field was good with a relative difference of less than $\pm 3 \%$ inside the field at any depth. In the low dose gradient, it increased up to $\pm 5 \%$. For a $20 \times 20 \mathrm{~cm}^{2}$ field size, the relative difference was less than $\pm 5 \%$ at any depth, for any point inside the field, and increased up to $-20 \%$ in the low dose region. Figure 5.5 shows the dose profiles of the wedged beams that represent the cross-plane profiles. Similar differences were found except for the $20 \times 20 \mathrm{~cm}^{2}$ field, where the relative difference was $-9 \%$ inside the field. However, the largest field size used in this thesis was $10 \times$ $10 \mathrm{~cm}^{2}$.

The results of the Linac model are in agreement with other studies that modelled the Elekta linear accelerator (Sheikh-Bagheri and Rogers, 2002, Almberg et al., 2012). 


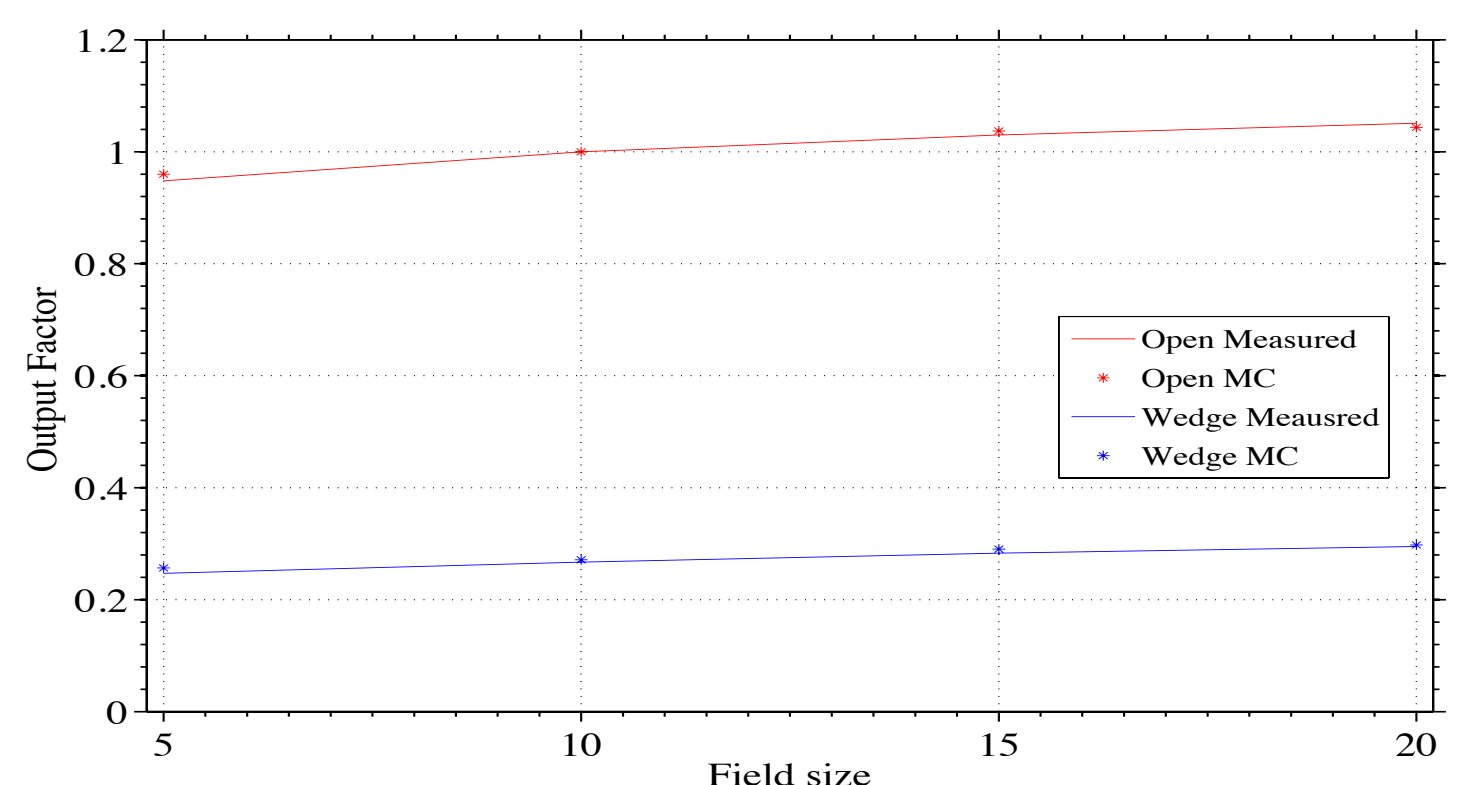

Figure 5.6: Output factors comparison between measurement (solid line) and simulation (dotted line) for different field sizes for open and wedged beams.

Using EGSnrc code, Sheikh-Bagheri et al (2002) suggested using 6.3 MeV with a $0.11 \mathrm{~cm}$ spot size. Almberg et al (2012) stated that $6.45 \mathrm{MeV}$ and an elliptical spot size of $0.025 \mathrm{~cm}$ in-plane, and $0.1 \mathrm{~cm}$ cross-plane, as the best fit for a $6 \mathrm{MV}$ photon beam using the Elekta Synergy Linac.

\subsubsection{Output factor and quality index comparison}

The $\mathrm{OF}$ at the maximum dose depth of $1.5 \mathrm{~cm}$ were compared for the measurement and simulation, for different field sizes, and with and without a wedge. This is shown in Figure 5.6. For open beams, the largest relative difference was found for a field size of $5 \times 5 \mathrm{~cm}^{2}$ where the simulation overestimated the OF by $1.26 \%$. This decreased down to $-0.66 \%$ for $20 \times 20 \mathrm{~cm}^{2}$ field size. For wedged beams, the largest relative difference was found for a $5 \times 5 \mathrm{~cm}^{2}$ field size where the simulation overestimated the OF by $3.92 \%$. The difference decreased down to $-0.88 \%$ for a $20 \times 20 \mathrm{~cm}^{2}$ field size.

Finally, the QI (or TPR ${ }^{20}{ }_{10}$ ) was compared. The QI is calculated by taking the ion chamber value at a depth of $20 \mathrm{~cm}$ and dividing it by the value at a depth of $10 \mathrm{~cm}$, for the same SCD and with a field size of $10 \times 10 \mathrm{~cm}^{2}$, with different SSDs. The measured QI for a $6 \mathrm{MV}$ beam was 0.675 and the QI for the simulation of the finetuned electron energy and spot size was 0.677 . 


\subsection{Conclusion}

This chapter has explained the modelling of the Elekta Synergy 6MV linear accelerator using BEAMnrc/DOSXYZnrc codes. This required tuning the Linac head model to adjust the appropriate mean energy and spot size of the incident electrons to find the best match with the measurement. Using a parallel Gaussian beam (Gaussian distributed in space $\mathrm{X}$ and $\mathrm{Y}$ ) with a monoenergetic electron beam (SOURCE 19, BEAMnrc), the fine-tuned parameters for the $6 \mathrm{MV}$ photon beam was found to be a $6.05 \mathrm{MeV}$ mean electron energy with a $0.14 \mathrm{~cm}$ circular spot size.

After the fine-tuned model was achieved, a comprehensive investigation of the model outputs was carried out in order to validate the MC Linac model. The simulation of the linacs was very CPU intensive, therefore a number of variance reduction techniques were used in order to speed up the simulation as much as possible. The validation process of the MC Linac model involved the comparison of PDD, dose profile, QI and OF, for open and wedged beams, with the measurement. The MC model was in agreement with the measurements for fields ranging from $5 \times 5$ to $20 \times 20 \mathrm{~cm}^{2}$. As a result, in the following chapters the validated MC Linac model will be used to produce clinical MC-based treatment plans and thus enable the separation of uncertainties arising in the dose calculations from the commercial treatment planning system algorithms, from the uncertainties introduced by the different scanning technologies (CT and CBCT). In the next chapter, the dose calculation accuracy based on modified/segmented CBCT images of a male pelvis phantom will be investigated. 
Chapter 6

CBCT-based dose calculation of a male pelvis phantom 


\subsection{Introduction}

The integration of CBCT into a Linac allows the possibility of imaging the patient in the treatment position immediately before, during or after treatment. These images can be used to correct patient set-up error and evaluate or monitor any changes in shape, size and position of the prostate and those related to weight loss, organ filling/distension or tumour regression. This can be achieved by registering CBCT images with the reference pCT images. In addition, CBCT images can be used for ART in various ways such as choosing a "plan of the day", generating a treatment plan and recalculating the dose distribution. However, CBCT images generated using the XVI system on the Elekta treatment units provide pixel values (gray scale) or CBCT numbers instead of true HUs or CT numbers. Compared with pCT, CBCT images contain more scatter and suffer from more image artefacts (see Section 2.5) due to the cone beam geometry and a larger detector. The image artefacts and scatter affect the pixel values and are dependent on the FOV, collimations, patient size and image acquisition parameters. Therefore, CBCT images cannot be used directly for dose calculation. The aim of this project is to correct CBCT data and convert the pixel values to true HUs or CT numbers that are comparable to the pCT, consequently, to enable quick dose calculations assessment to be performed with the use of CBCT and then use it as a quick-decision-making tool for re-planning. This will eliminate the need to rescan the patient in pCT and potentially avoiding additional imaging dose. Before that, the accuracy of the dose calculation on the corrected or processed images has to be investigated and compared with those of the pCT. In this chapter, a simple anthropomorphic geometry phantom was used and a simple dose distribution was performed and investigated.

\subsection{Methodology}

\subsubsection{Multiblock phantom}

A male pelvis phantom was built using the multiblock phantom that was designed by A. Seaby et al. (Swansea NHS Trust, Swansea, UK) (Seaby et al., 2002) (Figure 6.1). The phantom is made up of two tissue-equivalent materials: WT1 for water and IB7 for inner bone. The length of the phantom blocks is $30 \mathrm{~cm}$ and each block is a square shape or a triangle with sides of $4 \mathrm{~cm}$. The blocks were rearranged to represent 


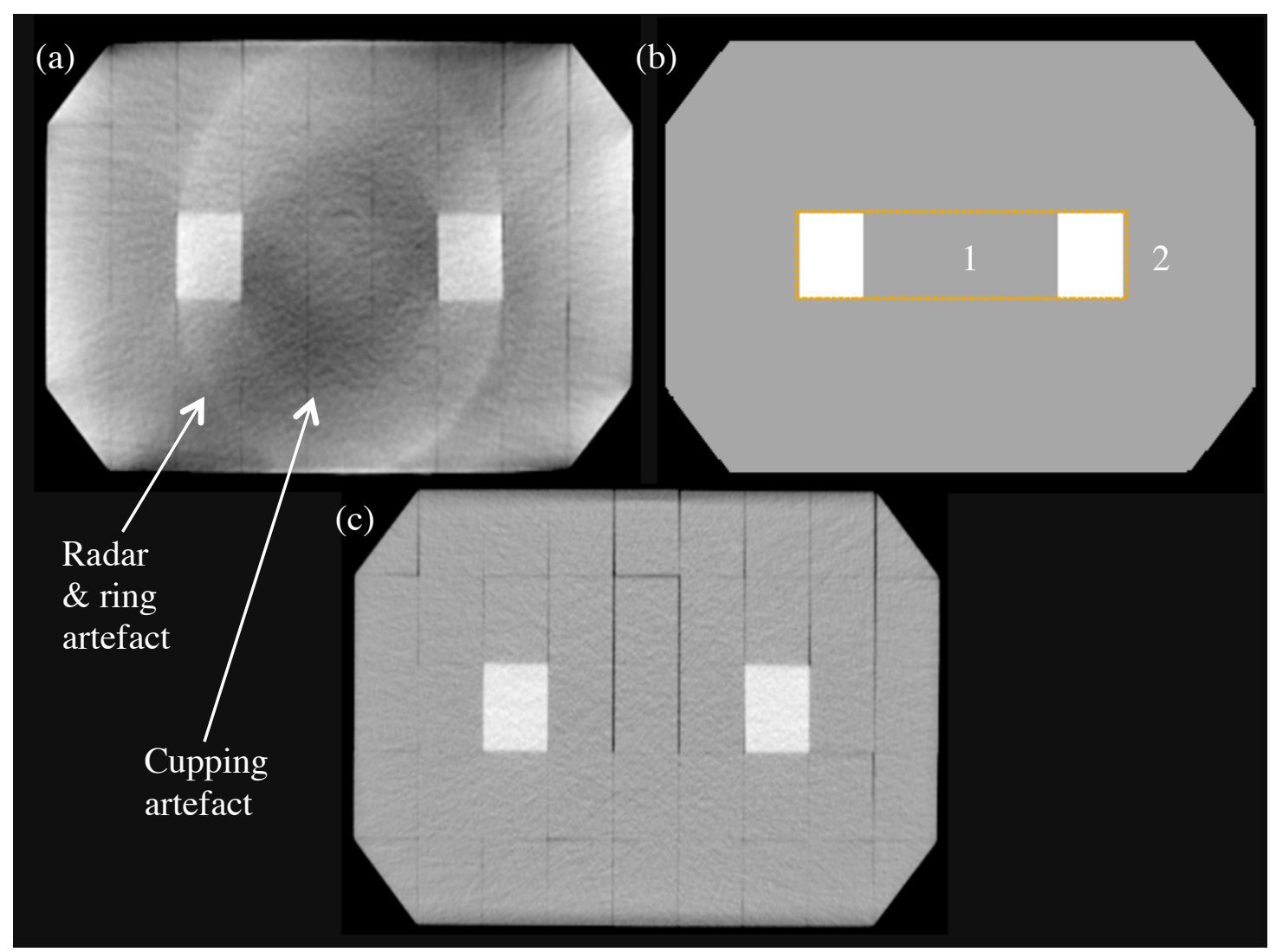

Figure 6.1: Multiblock phantom scan using (a) CBCT, (c) pCT and the resultant image after segmenting $C B C T$, sCBCT (b).

sections through the pelvic region as shown in Figure 6.1. The lateral separation of the pelvis was $36 \mathrm{~cm}$ and the length was $20 \mathrm{~cm}$ in anterior-posterior direction.

\subsubsection{CT and CBCT image acquisition}

The male pelvis phantom was scanned using a Philips Brilliance Big Bore CT scanner installed at Singleton Hospital (Philips Medical Systems, version 2.3, Cleveland, Ohio, USA). It is a 16-slice helical scanner with a bore diameter of $85 \mathrm{~cm}$. The CT images were acquired with $120 \mathrm{kVp}$ and the tube current was modulated during the scan based on phantom anatomy. The matrix size of the reconstructed images was 512 $\times 512$ and voxel sizes were $0.98 \times 0.98 \times 5 \mathrm{~mm}^{3}$. The scan length in the superiorinferior direction was $29.5 \mathrm{~cm}$.

A CBCT scan was acquired with a medium FOV (MFOV), and collimator M10 and the F1 filter using the following parameters: $120 \mathrm{kVp}, 32 \mathrm{~mA}, 40 \mathrm{~ms}$ per projection and 660 projections. The matrix size of the reconstructed images was $410 \times 410$ and 
the voxel size was $1 \times 1 \times 3 \mathrm{~mm}^{3}$ and the scan length in the superior-inferior direction along the phantom (G-T direction) was $12 \mathrm{~cm}$.

\subsubsection{Modification of CBCT images}

The main input for radiotherapy treatment planning is the CT image data which consists of pixels associated with CT numbers or HUs. These CT numbers are converted into relative ED by using HU-ED calibration in the TPS software for use in dose calculation algorithms (Figure 6.3). Since the CBCT images contain more scatter than a conventional CT system, the CT HU-ED calibration should be changed based on the CBCT image if the image is to be used for dose calculation. This cannot be done with the existing TPS software, OMP, where the CT calibration is intrinsic and cannot be altered by the user. In addition, the amount of scatter, thus image artefacts, depends on the FOV, collimator and object size (see Chapter 2). Consequently, even if multi-calibration (based on CT and CBCT) was achievable in OMP, one calibration curve that is based on CBCT would not be sufficiently accurate for dose calculation. Therefore, the CBCT images need to be corrected to accurately represent HUs or CT numbers that are similar to those in pCT images, thus fitting the HU-ED calibration in the TPS. This was achieved by converting the pixel values (CBCT numbers) of CBCT images into CT numbers used in the pCT. This step involved segmenting CBCT pixel values into different discrete bins, where a bin is a region of a uniform material with single CT number/HU, using MLT algorithm (Boggula et al., 2007, Boggula et al., 2009). The MLT algorithm used in this work was written in MATLAB (Mathworks, Natick, MA).

The CBCT images of the male pelvis phantom were imported and read in MATLAB using built-in (DICOMread) functions. The MLT algorithm was then performed to convert the pixel values that lie in predefined ranges into material bins or segments. The CBCT images were then segmented into only two material bins, by visualising the images, that represent water and inner bone, as shown in Figure 6.1(a). The CBCT image set was divided into two regions. For region 1, the range of pixel values for water was from 250 to 829 pixel value. The range is chosen by the user to choose whether the range is appropriate to represent a specific material bin with aid from the original CT. For inner bone, the range was from 830 to 4000 . Again, this is chosen by the user based on the artefacts and anatomical information and can be 


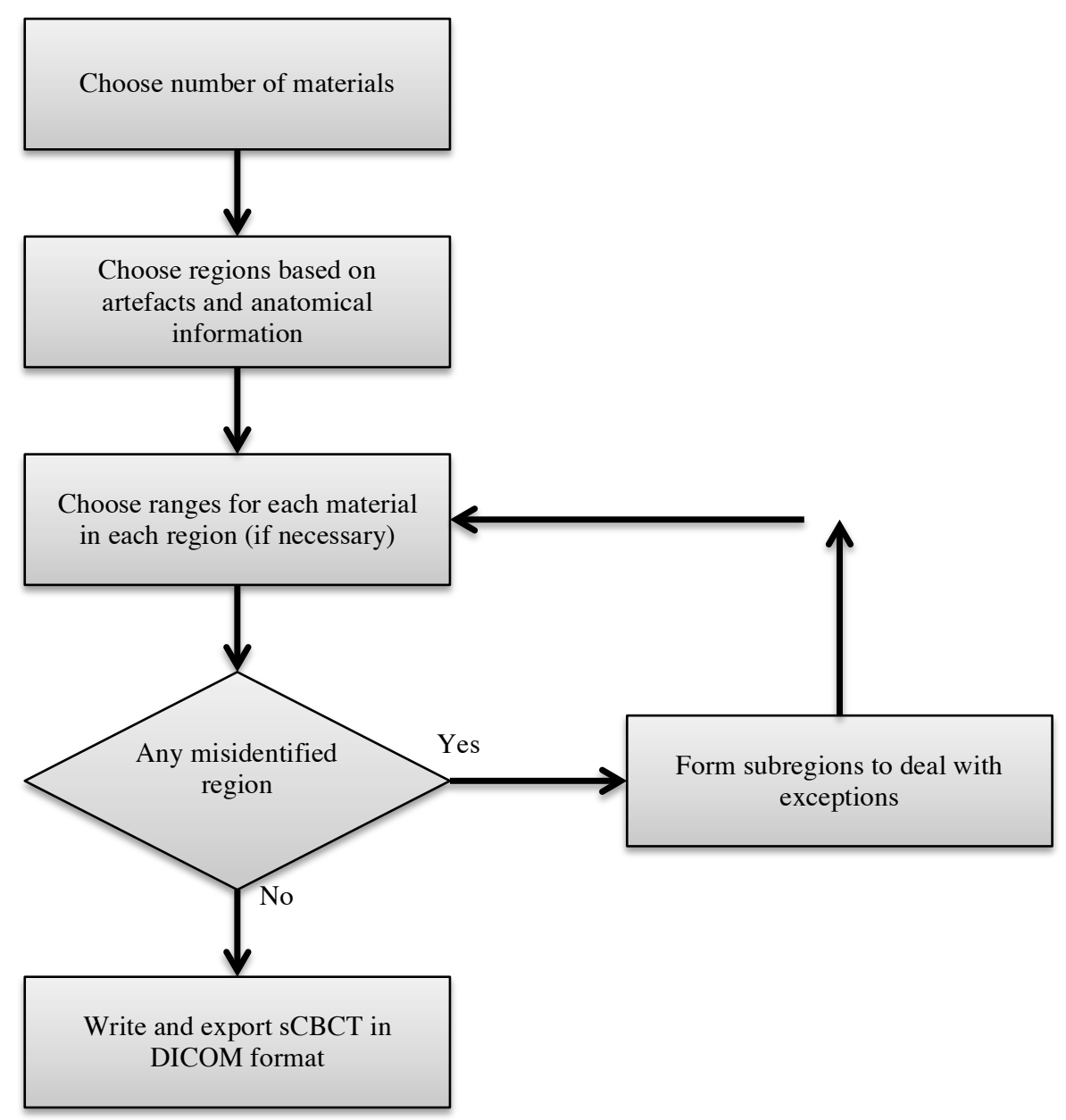

Figure 6.2: Segmentation of CBCT process using the MLT algorithm.

easily modified in the MLT algorithm. Therefore, any pixel with a value of more than 250 and equal or less than 829 was assigned as water and given a value of 1024 (0 $\mathrm{HU})$. Any pixel with a value of more than 829 was assigned as inner bone 1195 (171 $\mathrm{HU})$, as in the pCT images. These assigned values were chosen based on the CT ramp used by the TPS. For region 2, the range of pixel values for water was from 250 to the maximum value (4000). This was performed through all slices. If any water region is erroneously replaced by inner bone in any slice (and vice versa) it can be manually and individually corrected by applying the MLT algorithm to a subregion of the CBCT. Finally the corrected or segmented CBCT images, referred to as SCBCT, were written and exported from MATLAB in a digital imaging and communication in medicine (DICOM) format with the original voxel dimensions and DICOM information and headers. Figure 6.2 shows a flowchart that describes the process briefly. As a result, it can be seen that the segmentation method was able to remove 


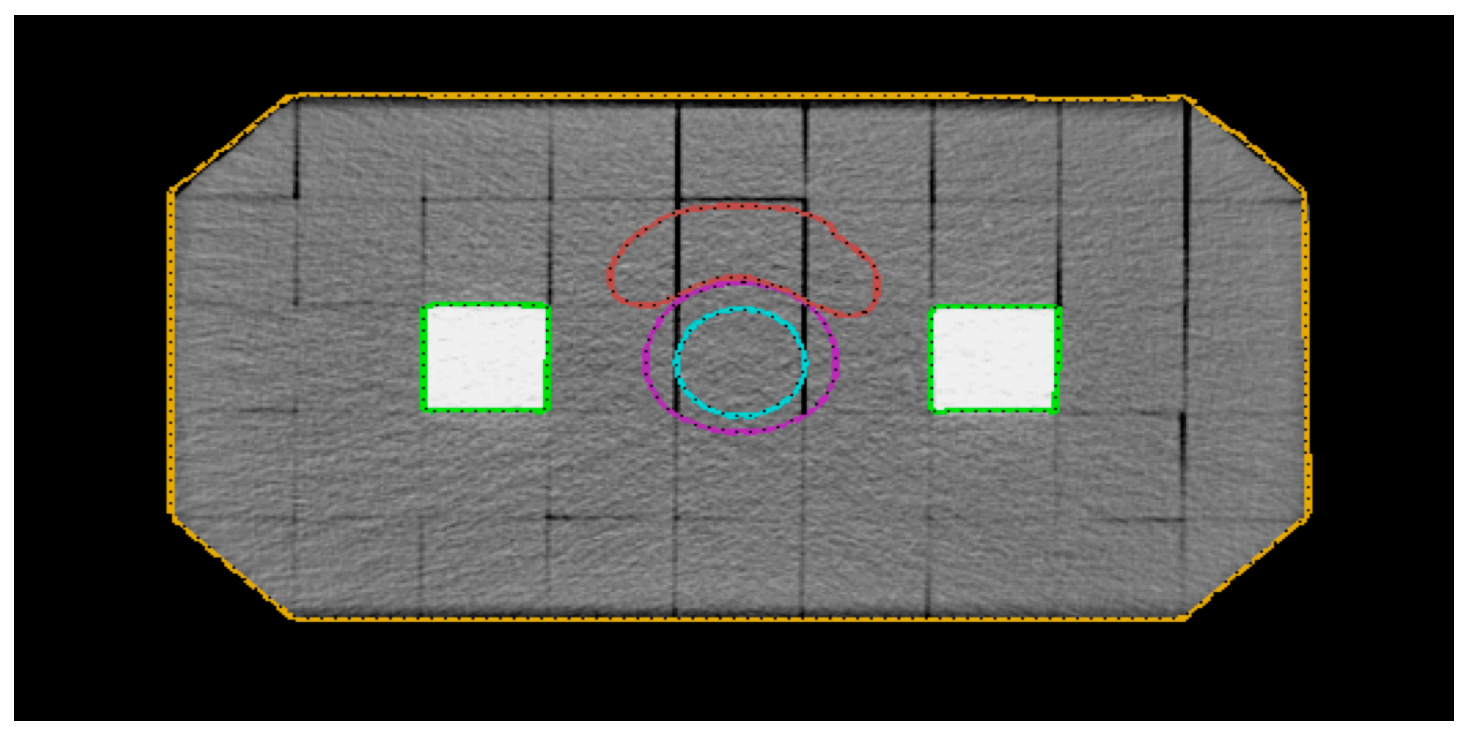

Figure 6.3: The pCT of the multiblock phantom with the drawn structures.

image artefacts, such as cupping, ring and radar artefacts, as shown in Figure. 6.1(b), which would also affect the accuracy of the dose calculation.

\subsubsection{Treatment planning system and treatment plan}

Both CT and CBCT images were transferred to the TPS via DICOM protocol for dose calculation. In OMP TPS, a complete 3D dose distribution of a treatment plan can be displayed and evaluated using DVH. It supports two types of algorithm to calculate dose distribution; PB and CC algorithm, which are based on pencil and point kernels, respectively (see Chapter 3 ).

A field-in-field radiotherapy plan was generated from the CT of the phantom using a $6 \mathrm{MV}$ photon beam energy. The plan consisted of six fields: two anterior beams, 10 and $8 \mathrm{~cm}^{2}$ field size; two left lateral beams, 10 (with $45^{\circ}$ wedge) and $8 \mathrm{~cm}^{2}$ field size; and two right lateral beams, 10 (with $45^{\circ}$ wedge) and $8 \mathrm{~cm}^{2}$ field size. The wedge in the Elekta Synergy linear accelerator is a motorized wedge that can be positioned in or out the beam and the desired angle can be achieved by combining an open and wedge beam $\left(0^{\circ}\right.$ to $\left.60^{\circ}\right)$. Structures were drawn in the pCT to represent CTV, PTV, femoral heads, rectum and bladder as in a real patient, as shown in Figure 6.3. The CTV was grown with a $1 \mathrm{~cm}$ anterior/left/right/superior/inferior margin and $0.5 \mathrm{~cm}$ posterior margin to give the PTV. The CBCT and pCT images were fused using ProSoma virtual simulation software (v3.3, MedCom, Germany) and the structure sets were then transferred to both $\mathrm{CBCT}$ and sCBCT images. The plan was then copied to 
CBCT (without any modification on the image set) and sCBCT using the same geometry and monitor unit (MU) values and the prescription dose was 74 Gy in 37 fractions (2 Gy/fraction) with the isocentre was positioned inside the PTV. The doses were recalculated in OMP using PB and CC algorithms. The reason of using different dose calculation algorithms is to allow comparison with the MC technique and to see the effect of CT number on the dose calculation across various algorithms to highlight where any differences may occur. Thus any uncertainty inherited from the dose calculation algorithms in OMP can be distinguished from that associated with the use of different image sets.

\subsubsection{Monte Carlo-based treatment plan}

The MC plan was performed using DOSXYZnrc user code using the 'BEAM treatment head simulation' option as a radiation source. In this option, the simulation started from the initial electron source in the Linac model that was built using BEAMnrc (see Chapter 5). Then the particles were transported from the Linac into the male pelvis phantom and the dose was scored.

The transport parameters and variance reduction techniques were used as in Table 5.2 in Chapter 5. The global photon and electron cutoff energy, PCUT and ECUT, were $0.01 \mathrm{MeV}$ and $0.70 \mathrm{MeV}$, respectively. As the male pelvis phantom is not a homogenous phantom, the 'HOWFARLESS' option was turned off in DOSXYZnrc. This made the simulation time longer as the simulation of each history was interrupted at each voxel boundary to take into account any changes in the material densities.

The treatment plan parameters, such as beam energy, collimators positions, wedge, isocentre coordinates, SSD, gantry angle and collimator angle, were extracted from the OMP system using an in-house Excel macro (written by Mark Edwards). A single input file, for each BEAMnrc and DOSXYZnrc, was created for each beam manually based on these parameters. The coordinate difference between the OMP and MC system was taken into account. Each beam was run separately with $55 \times 10^{6}$ histories, chosen so as to reduce the statistical uncertainty to less than $2 \%$ in the calculated dose at the plan isocentre. 


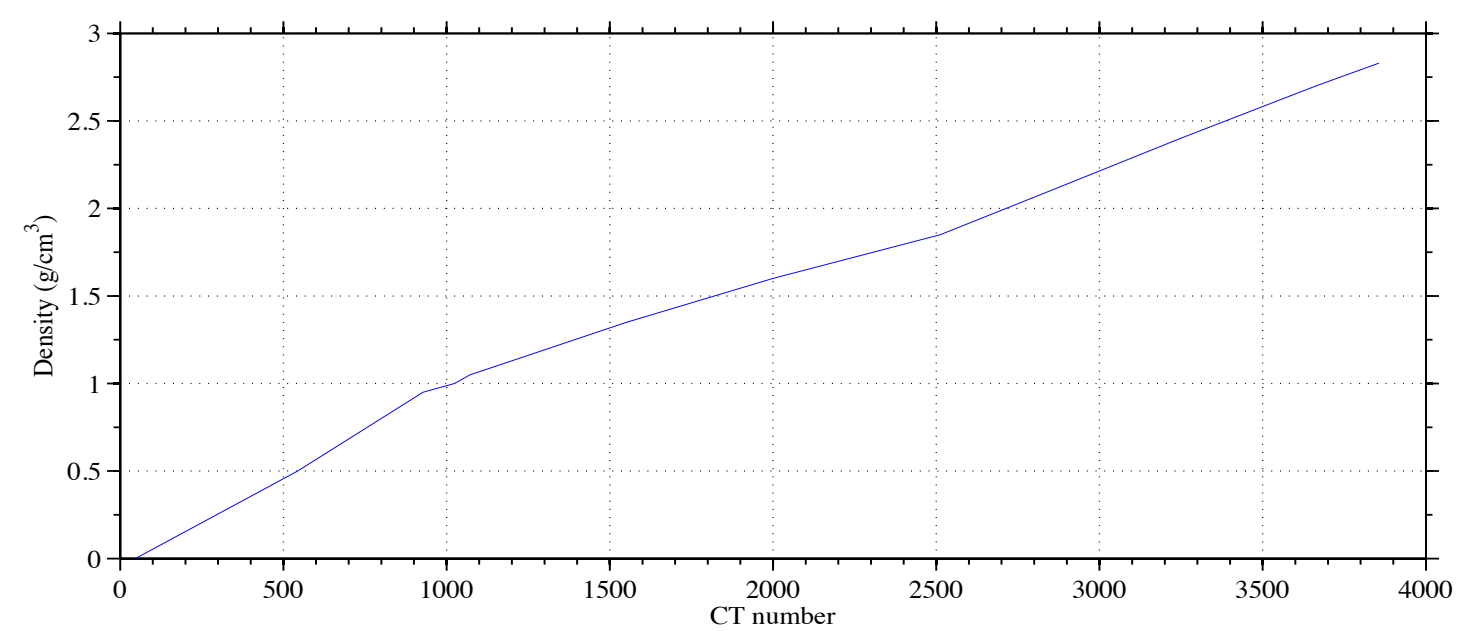

Figure 6.4: The CT ramp for the conversion of CT values to material type and density used in $O M P$. The same ramp was used in MC. The density and composition of the material used in this ramp were included in the PEGS4 cross section data file.

The MC calculations were run on High Performance Computing (HPC-Wales), a cluster of computers, to speed up the calculation (HPC-Wales). The simulation of each beam was split into 100 jobs using 100 nodes, which were run in parallel. Thus the simulation time was decreased by a factor of 100 . Then, at the end of the simulation of each beam, the calculated dose distributions from all jobs were combined in a single format file called 3ddose, which was then weighted by their MUs using MATLAB scripts. Finally, the MC plan was generated by combining the dose distributions of all weighted beams (open and wedged) in a single 3ddose file using a MATLAB script. The final dose was then normalized at the isocentre to 74 Gy.

\subsubsection{Conversion of CT images into MC format}

In order to perform $\mathrm{MC}$ dose calculations on the $\mathrm{CT}, \mathrm{CBCT}$ and $\mathrm{SCBCT}$ phantom images in DOSXYZnrc, each DICOM image set had to be converted to a compatible format. This was done by using the DICOM-RT toolbox (dicomrt_ctcreate function) which is provided with a software called Computational Environment for Radiotherapy Research (see Section 6.2.7) (Spezi et al., 2002, Deasy et al., 2003). 


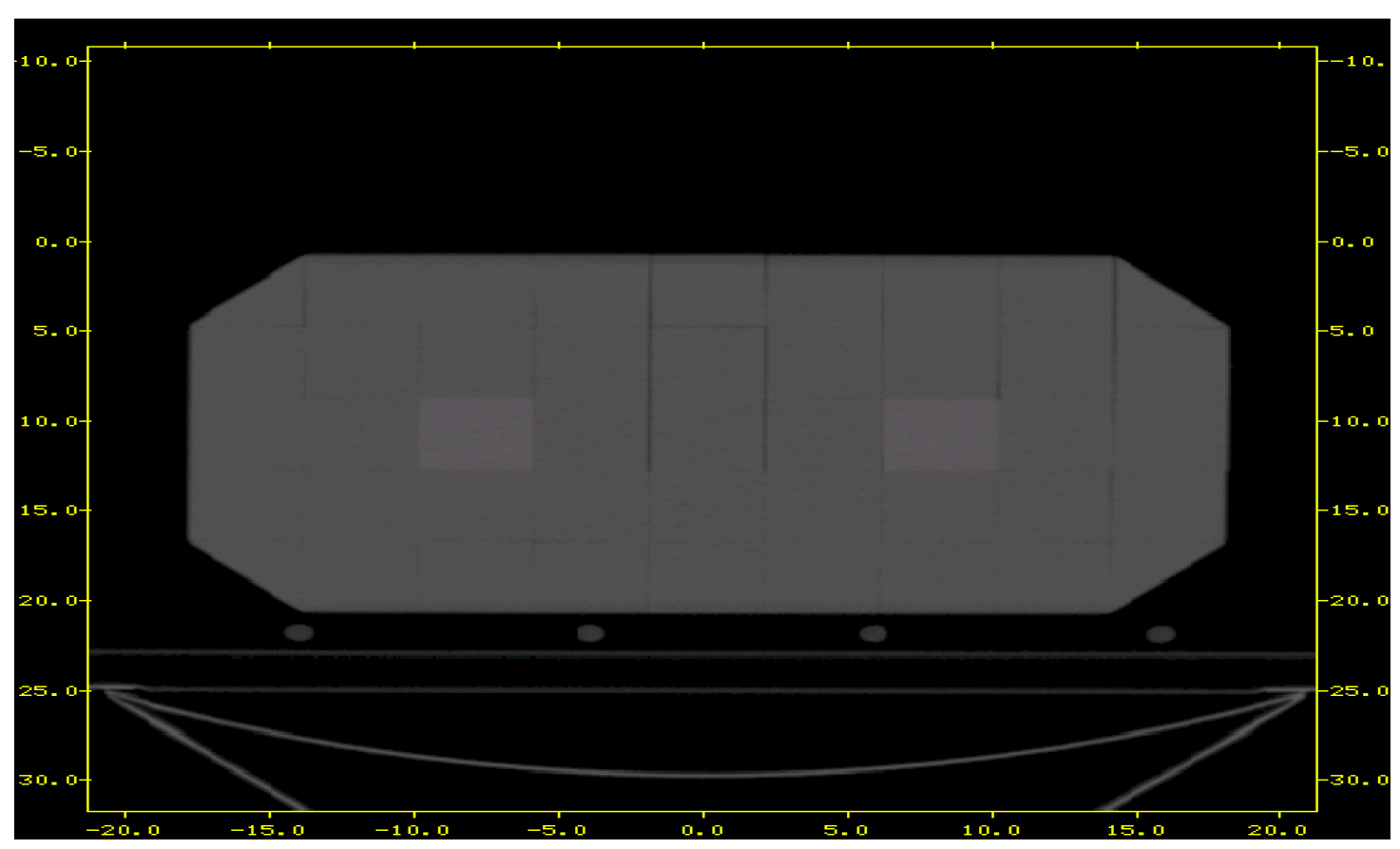

Figure 6.5: A CT transverse slice of the Multiblock phantom in egsphant format (generated using DOSXYZ_show utility, provided with EGSnrc package).

In the dicomrt_ctcreate function (equivalent to the CTcreate user code provided with the EGSnrc package), each CT number in the DICOM images was converted to a specific material that must be included in the PEGS (preprocessor for EGSnrc) data file. This contains the atomic composition and cross section data of all materials involved in the simulation. Therefore, the atomic compositions and cross sectioal data of the male pelvis phantom materials, WT1 and IB7, were calculated and included in the PEGS file (see Table 6.1). However, the conversion was based on the same HUED calibration (CT ramp) as in the OMP software, as shown in Figure 6.4. Any voxel with a CT number higher than 3856 is considered as Iron with density of $7.87 \mathrm{~g} / \mathrm{cm}^{3}$. This conversion requires some input parameters such as material density lower bound, material density upper bound and material CT upper bound. The DICOM images were thus converted to different material matrices, of known atomic composition and density, through their relative electron densities. Finally, the converted data of each DICOM image set was written and exported in a format called egsphant, which was then used and read in DOSXYZnrc for dose calculation, with the same original voxel dimensions (Figure. 6.5). 
Table 6.1: Element composition of male pelvis phantom tissue substitutes.

\begin{tabular}{|c|c|c|c|c|c|c|c|}
\hline \multirow{2}{*}{ Tissue } & \multicolumn{5}{|c|}{ Element composition (\% by weight) } & \multirow{2}{*}{$\begin{array}{c}\text { Density } \\
\left(\mathbf{g} / \mathbf{c m}^{3}\right)\end{array}$} \\
\cline { 2 - 7 } & $\mathrm{H}$ & $\mathrm{C}$ & $\mathrm{N}$ & $\mathrm{O}$ & $\mathrm{Cl}$ & $\mathrm{Ca}$ & \\
\hline WT1 & 8.1 & 67.72 & 0.24 & 19.8 & 0.1 & 2.3 & 1 \\
\hline IB7 & 6.86 & 59.1 & 2.08 & 24.12 & 0.12 & 5.09 & 1.18 \\
\hline
\end{tabular}

\subsubsection{Treatment planning evaluation and comparison}

The pCT, CBCT and sCBCT plans were exported from the OMP system as the DICOM-RT files that were then imported into the computational environment for radiotherapy research (CERR) software. CERR is an open source software environment that is based on MATLAB (Deasy et al., 2003). Once the DICOM-RT was imported, the combined MC dose file was imported into MATLAB and CERR then overlayed it upon the DICOM images. CERR was then used to visualize and evaluate the resultant dose distributions of both the OMP and MC plans.

\subsubsection{DVH and dose profile comparison}

The cumulative DVHs were generated for PTV, rectum and bladder structures and compared with pCT, CBCT and sCBCT plans for PTV, rectum and bladder. The comparison of DVHs was performed using OMP, PB and CC, and MC algorithms. The dose profiles at the reference point (isocentre) depth were compared and plotted against the pCT, CBCT and sCBCT numbers to show the dose distribution of CBCT and sCBCT profiles relative to the reference $\mathrm{pCT}$ profile/number. The relative difference between $\mathrm{pCT}$ and $\mathrm{sCBCT}$ dose profiles was plotted to aid comparison, which is defined as Drel $=($ DCB-DCT $) /$ DCT.

\subsubsection{Gamma index comparison}

The differences between pCT and CBCT/sCBCT dose distributions at the pixel level would be an accurate evaluation. However, superimposing dose distributions and calculating the differences does not allow for an accurate quantitative comparison because in high dose gradient regions, a small spatial difference can lead to a large 
dose difference. To allow an accurate quantitative comparison, gamma $(\gamma)$ index evaluation method was performed, which uses two separate criteria; dose difference at a certain point and the distance-to-agreement (DTA) value to determine the acceptability of the dose calculation (Low et al., 1998). The DTA is defined as the distance from one point in the reference dose distribution $\left(\mathrm{x}_{\mathrm{r}}\right)$, to the closest point in the calculated dose distribution $(\mathrm{x})$ of the same dose. The criteria were $3 \%$ dose difference (DD), and $3 \mathrm{~mm}$ DTA and 5\% low dose threshold. The pCT dose distribution was set as a reference. The $\gamma$ index evaluation is calculated using the following

$$
\gamma=\sqrt{\frac{\Delta x^{2}}{D T A^{2}}+\frac{\Delta D^{2}}{D D^{2}}}
$$

where $\Delta x=\left|x-x_{r}\right|$ and $\Delta D=D(x)-D\left(x_{r}\right)$ is the difference between dose values on the calculated and reference distributions (Low et al., 1998). If the $\gamma$ index value is below or equal to 1 , the calculation point passes the test, when the $\gamma$ index value is above 1 it fails. The $\gamma$ index evaluation was calculated inside the PTV, rectum and bladder structures. The $\gamma$ index evaluation is a regularly used tool in the radiotherapy community to compare plans or compare planned and delivered dose.

\subsection{Results and discussion}

It is worth stating the effects of some factors that are relevant to the results of this thesis. As mentioned in Chapter 2, the CBCT images contain more scatter than conventional CT, which increases with FOV, collimations and object size, and varies with the acquisition parameters such as $\mathrm{kVp}$, number of projections and mAs. Consequently, it is essential to study the stability of CBCT numbers. The stability of CBCT numbers of the XVI system at Singleton hospital was examined over four consecutive weeks in a Master's project using the Catphan phantom (The Phantom Laboratory, Salem, New York, USA) (Almatani, 2011). The results confirmed the stability of CBCT with time (within $4.1 \%$ ) and were in agreement with other studies (Hu et al., 2010). The results also showed that the CBCT numbers were highly affected by the FOV size and, less so, the collimator size within a specific FOV, and object size. In addition, the scan parameters showed a significant impact on the CBCT numbers. These findings were in agreement with other studies (Richter et al., 2008, 
Lu et al., 2010, Kamath et al., 2011).

The stability of CBCT numbers is particularly important if the CBCT images are used for dose calculation using a HU-ED calibration curve, as variations in CBCT HU-ED calibration curves can lead to dose differences of up to $17 \%$ (Takemura et al., 2015). In this thesis, the dose calculation was performed in CBCT images that were modified/segmented to represent CBCT numbers that are similar to CT numbers in the pCT, thus an additional HU-ED calibration curve was not needed. In addition, the stability of CBCT number is relatively unimportant as the CBCT images contain only two different materials and the phantom size is much smaller than the FOV. Thus the amount of scatter is small and, consequently, its effect on the CBCT numbers is also small. Therefore, the segmentation of CBCT into different materials would not be affected by the relative instability of CBCT numbers.

The impact of scans parameters on the CBCT number is not considered in this thesis as only two scan protocols are used at Singleton hospital for the pelvic region. The only difference between the two protocols is the collimation size, M10 and M15. The rest of the parameters are the same. Increasing the collimator size from M10 to M15 resulted in a very small reduction in the CBCT numbers, due to a slight increase in the image noise, and cupping artefacts, due to the increased amount of scatter (Almatani, 2011). Therefore, such a reduction would not affect the performance of MLT algorithm in this thesis.

\subsubsection{DVH and dose profile comparison}

Figure 6.6(a) shows the DVH of a prostate field-in-field plan with a prescription dose of $74 \mathrm{~Gy}$ in 37 fractions. It shows the dose of the $\mathrm{pCT}, \mathrm{CBCT}$ and SCBCT plans to the PTV, rectum and bladder using the PB algorithm. The largest differences were found when the CBCT images were used directly for dose calculation without segmenting or converting the CBCT numbers to CT numbers/HUs, similar to those in pCT images. In terms of PTV coverage, the relative dose difference in $\mathrm{D}_{\text {mean }}$ between the pCT and CBCT plans was $8.3 \%$ and in $\mathrm{D}_{\min }$ was $13.9 \%$ when using $\mathrm{PB}$ algorithm, as shown in Table 6.2. For OARs, the CBCT plan overestimated the rectum minimum dose by $26.1 \%$ compared with the pCT plan. Similar results were found when using CC and MC algorithms, as shown in Figure 6.6(b) and Figure 6.6(c), respectively. 

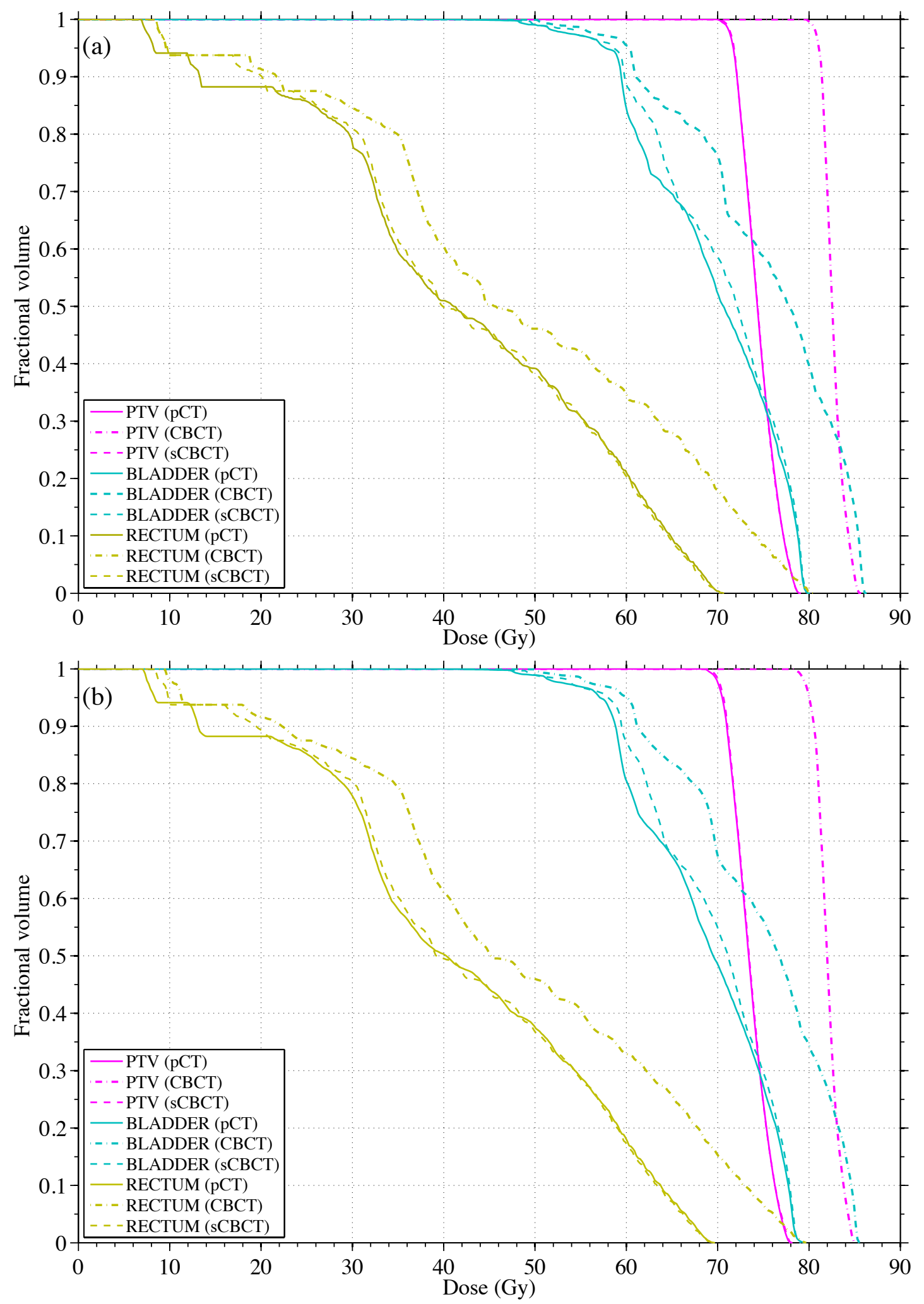


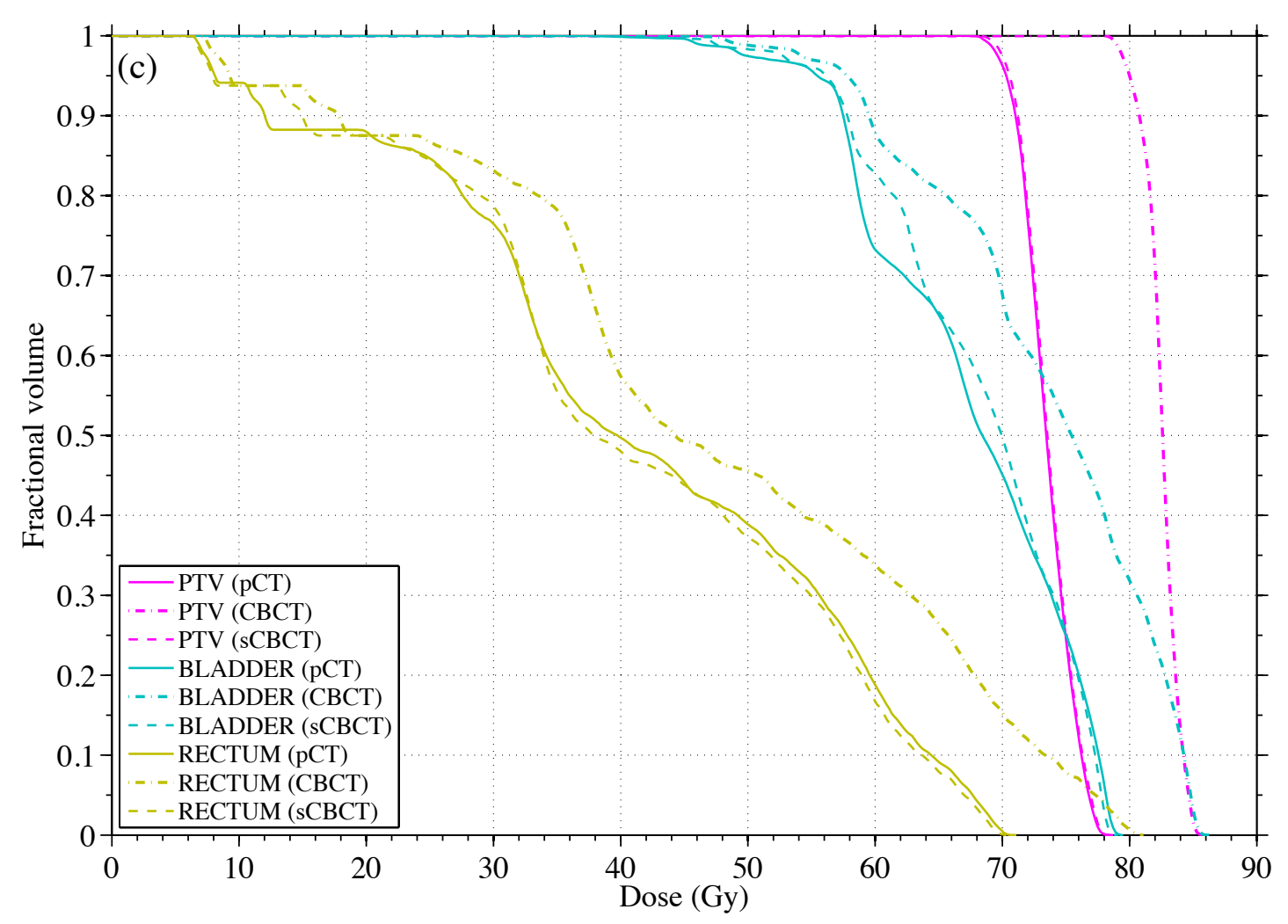

Figure 6.6: DVHs comparison between $p C T, C B C T$ and $s C B C T$ plans for PTV, rectum and bladder using (a) PB, (b) CC and (c) MC algorithm (prescription dose $74 \mathrm{~Gy}$ ).

On the other hand, the dose differences greatly decreased when the CBCT images were segmented into regions and the CBCT numbers were converted to CT numbers (sCBCT numbers), as shown in the sCBCT plan. It can be clearly seen that the sCBCT plan is in good agreement with the pCT plan. In general, the dose differences between pCT and sCBCT plans in all structures were less than $\pm 2 \%$ when using all algorithms. These results were similar to previous studies (Boggula et al., 2007, Onozato et al., 2013, Onozato et al., 2014). The only exception was the rectum minimum dose which was $26.1 \%, 19.7 \%$ and $3.2 \%$ when using $\mathrm{PB}, \mathrm{CC}$ and $\mathrm{MC}$ algorithms, respectively. The reason of this difference may be due to the gaps between the blocks when the phantom was setup in the CT scanner (Figure 6.3 and Figure 6.5). These gaps were seen in the CBCT images as well but were converted to a water material bin in the SCBCT. The difference was small when using the MC algorithm compared with PB and CC algorithms because the gaps were converted to water in the pCT images as well.

In general, the PB algorithm was less accurate, as expected, at accounting for the differences between $\mathrm{CBCT}$ and $\mathrm{sCBCT}$ numbers, i.e. the rectum minimum dose was 
constant (Table 6.2). This is due to the fact that the PB algorithm calculates dose to water, thus, the fluctuation in the $\mathrm{pCT}$ number in the $\mathrm{pCT}$ images was not taken into account (see Chapter 3). In the sCBCT images, there was no variation in the sCBCT numbers, consequently, the PB algorithm dealt with both scans identically in some cases. On the other hand, the MC algorithm showed a significant reduction in the rectum minimum dose difference compared with the $\mathrm{pCT}$ plan when moving from the CBCT to the sCBCT plan. Even though CC and MC algorithms calculate dose to medium, the difference with the PB algorithm in such a simple geometry phantom is small. Thus all algorithms would be expected to perform in a similar manner.

Table 6.2: Dose and coverage differences between CBCT/sCBCT plans and the $p C T$ plan, in $\%$, for the PTV, rectum and bladder.

\begin{tabular}{ccccc|ccc} 
& & \multicolumn{3}{c}{ CBCT } & \multicolumn{3}{c}{ sCBCT } \\
\cline { 2 - 8 } & & PB & $\mathbf{C C}$ & MC & PB & CC & MC \\
\hline \multirow{3}{*}{ PTV } & $\mathbf{D}_{\text {max }}$ & 8.34 & 8.96 & 9.41 & 0 & 0.25 & -0.25 \\
\cline { 2 - 8 } & $\mathbf{D}_{\text {mean }}$ & 10.96 & 11.61 & 12.25 & 0.02 & 0.06 & 0.16 \\
\cline { 2 - 8 } & $\mathbf{D}_{\text {min }}$ & 13.98 & 14.26 & 16.09 & 0.28 & 0.29 & 1.19 \\
\hline \multirow{3}{*}{ Rectum } & $\mathbf{D}_{\text {max }}$ & 13.86 & 14.34 & 14.06 & -0.56 & -0.28 & -1.12 \\
\cline { 2 - 8 } & $\mathbf{D}_{\text {mean }}$ & 15.22 & 16.19 & 14.47 & 1.25 & 1.24 & -0.67 \\
\cline { 2 - 8 } & $\mathbf{D}_{\text {min }}$ & 26.08 & 33.8 & 15.87 & 26.08 & 19.71 & 3.17 \\
\hline \multirow{3}{*}{ Bladder } & $\mathbf{D}_{\text {max }}$ & 7.74 & 7.27 & 8.55 & -0.24 & -0.75 & -0.5 \\
\cline { 2 - 8 } & $\mathbf{D}_{\text {mean }}$ & 8.55 & 8.91 & 8.99 & 1.13 & 1.2 & 1.04 \\
\cline { 2 - 8 } & $\mathbf{D}_{\text {min }}$ & 11.3 & 14.53 & 16.08 & 0.49 & 2 & 1.07 \\
\hline
\end{tabular}

Figure 6.7 shows the cross-plane profile of pCT, CBCT and SCBCT at the depth of the plan isocentre as well as the pCT, CBCT and sCBCT numbers at that depth. Figure 6.7(a), (b) and (c) represents the dose profiles using PB, CC and MC algorithms, respectively, each with a subplot that aids a clear comparison between pCT and sCBCT dose profiles. It can be clearly seen that the sCBCT numbers/dose profiles are in good agreement with the $\mathrm{pCT}$ numbers/dose profile. On the other hand, the CBCT numbers were much lower than the CT number, especially at the centre of the phantom. This is due to cupping artefacts caused by beam hardening and scatter 

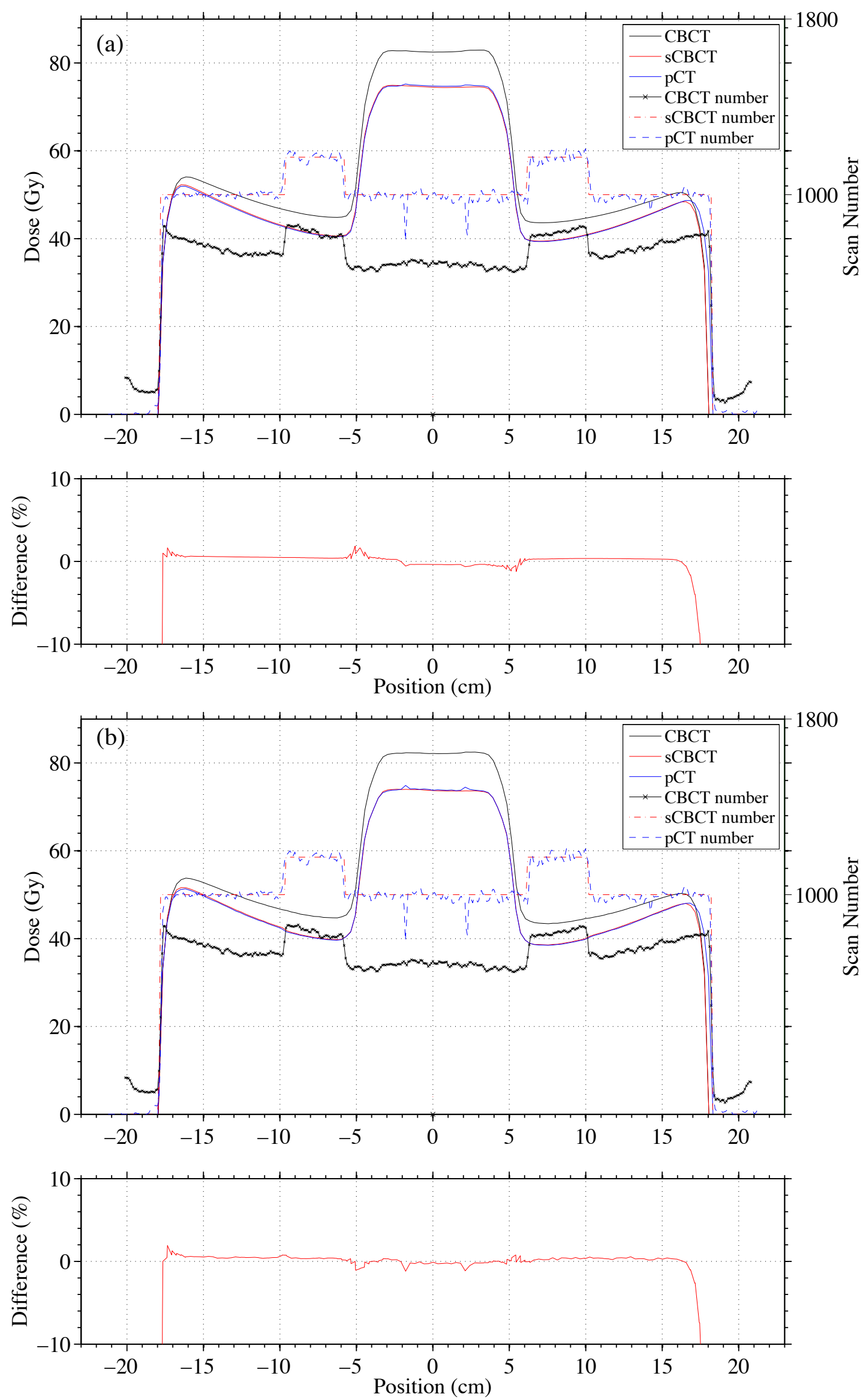

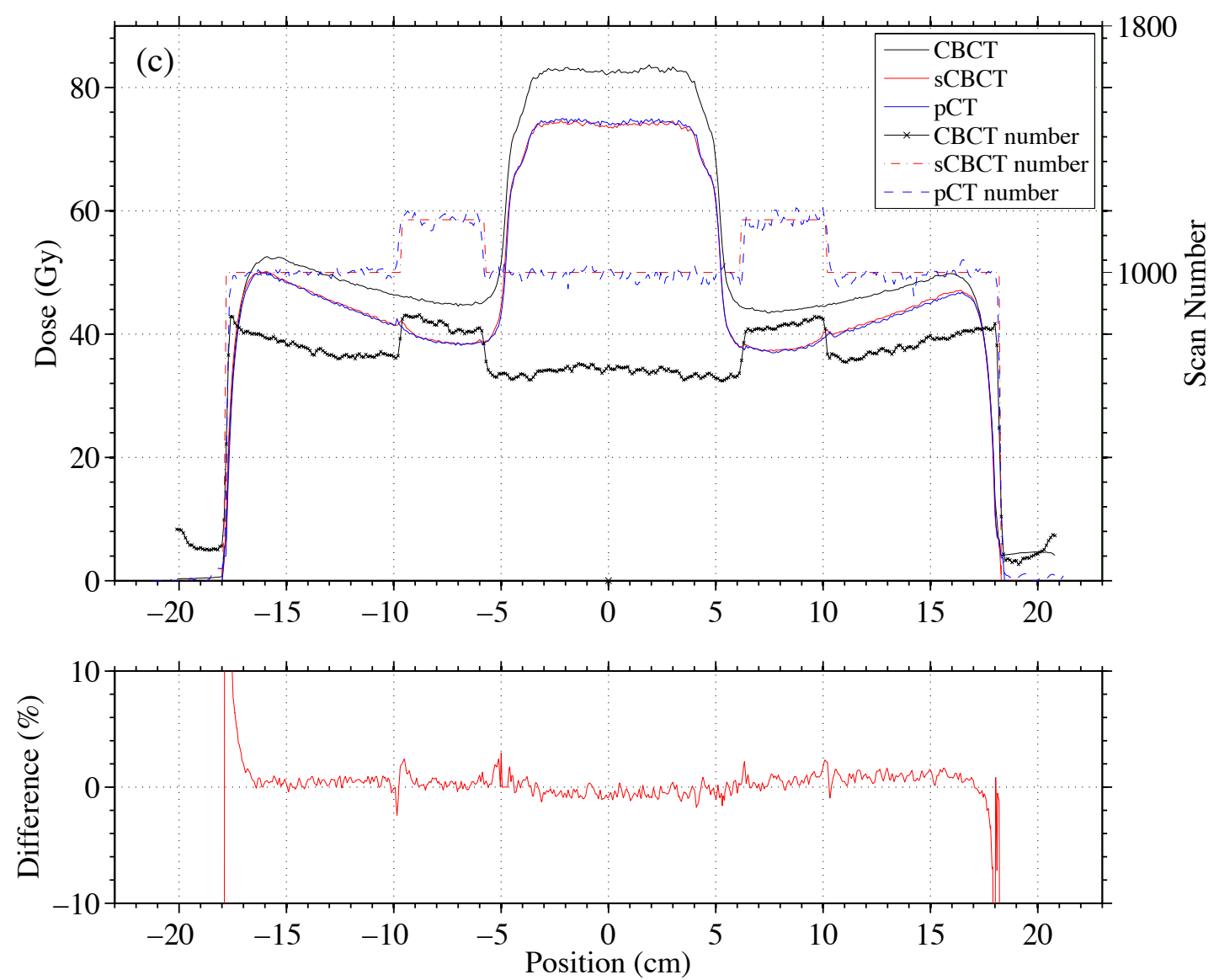

Figure 6.7: Comparison of the dose profile of $p C T, C B C T$ and $s C B C T$ plans at the isocentre depth using (a) $P B$, (b) $C C$ and (c) MC algorithm. The second y axis represents the CBCT number, $s C B C T$ number and $C T$ number. Subplots represent the differences between $p C T$ and sCBCT dose profiles.

that lead to a drop in the CBCT number in the centre compared with the periphery region (Figure. 6.1(a)). Consequently, the calculated doses were much higher compared with the calculated doses based on the pCT images. The segmentation of CBCT using the MLT algorithm was able to remove cupping artefacts, as shown in Figure 6.1(b). It corrected ring artefacts, caused by miscalibrated detector pixel lines/rows, elements, or manufacturing defects at a fixed location in the FPD as well as radar artefacts that appear as a circular radar bright-shaded region owing to inconsistencies in detector signal and/or gain (see Chapter 2).

However, in Figure 6.7(a) and (b), the drops in the pCT numbers in the high dose (PTV) region were caused by the gaps between blocks in pCT images. In this region, the dose difference between $\mathrm{pCT}$ and $\mathrm{sCBCT}$ was $\pm 0.5 \%$ using the $\mathrm{PB}$ algorithm whilst the CC algorithm was more sensitive and showed a difference of $\pm 1.2 \%$. These 
gaps were in the CBCT images as well but the increased amount of scatter increased the CBCT numbers in these regions in some slices. For MC calculations, these gaps in the pCT images were considered as water as shown in Figure 6.7(c). At the bone regions, some adjacent pixels were considered as water and some were considered as inner bone in the sCBCT images due to the threshold process. This was most observed by the MC algorithm, which showed a dose difference of $\pm 2.5 \%$ compared with the pCT dose profile (Figure 6.7(c)). This may be due to the statistical uncertainty of $\mathrm{MC}$ which was within $2 \%$. Due to the threshold nature, the lateral dose difference between pCT and sCBCT was more than $\pm 23 \%$ using all algorithms. This was excluded from each subplot to show clear dose differences at the PTV and inhomogeneities (bone) regions. However, the relative dose difference pCT and CBCT (in the high dose region) decreased from more than $10 \%$ to less than $-1 \%$ after the segmentation, which is similar to previous studies (Boggula et al., 2007).

Table 6.3: Summary of the $\gamma$ index with fixed DTA $=3 \mathrm{~mm}$ and $D D=3 \%$ for the calculation points falling inside the PTV, rectum and bladder, showing the percentage of points resulting with $\gamma \leq 1$.

\begin{tabular}{c|ccc|ccc} 
& \multicolumn{3}{|c|}{ CBCT } & \multicolumn{3}{c}{ SCBCT } \\
\cline { 2 - 7 } PTV & PB & CC & MC & PB & CC & MC \\
\hline Rectum & 0 & 0 & 0 & 100 & 100 & 100 \\
\hline Bladder & 29.49 & 33.69 & 49.45 & 100 & 100 & 100 \\
\hline
\end{tabular}

\subsubsection{Gamma index comparison}

Table 6.3 shows the $\gamma$ index evaluation for the calculation points falling inside the $\mathrm{PTV}$, rectum and bladder for $\mathrm{CBCT}$ and $\mathrm{sCBCT}$ plans using $\mathrm{PB}, \mathrm{CC}$ and $\mathrm{MC}$ algorithms. The results are shown as the percentage of calculation points resulting with $\gamma \leq 1$. As expected, all the calculation points in the PTV of the CBCT plan failed the test, when using all algorithms, showing $\gamma>1$. For the rectum region, more than $50 \%$ of the calculation points failed the test. For the bladder region, the lowest percentage of calculation points that passed the test was $20.9 \%$ when using the MC algorithm. The sCBCT plan, however, showed much better results where all the 
calculation points in the PTV, rectum and bladder regions passed the test using all algorithms, except $0.8 \%$ of the calculation points in the bladder which failed when using the MC algorithm. These results are in agreement with the previous studies (Boggula et al., 2009). As a result, these $\gamma$ values are considered to be clinically acceptable when the passing rate is greater than $95 \%$ with $3 \mathrm{~mm}$ DTA and 3\% DD criteria (Son et al., 2015).

\subsection{Conclusion}

In order to use CBCT images for dose calculations, the CBCT numbers or pixel values need to be converted to CT numbers or HUs that are similar to those in the pCT images. In this study, it has been demonstrated that if the CBCT images are used directly for dose calculation, even for a simple phantom, it results in a dose difference of more than $10 \%$ when compared with pCT images, and more than $70 \%$ of the calculation points failed $\gamma$ test. The segmentation of CBCT into two materials (water and bone) and converting the CBCT numbers to CT numbers using the MLT algorithm improves the relative dose accuracy by $8 \%$. This method is independent of CBCT image artefacts in contrast to the use of a CBCT calibration curve which is still subject to image artefacts. As a result, the use of CBCT images directly for dose calculation (without segmentation) was not performed in the rest of this thesis, as more complex geometries were used and the dose differences would be much higher than the dose difference in the phantom case. The next step is to correct and segment CBCT images of a more realistic patient type geometry, as presented in the next chapter, and then, even in challenging circumstances as, for prostate patients with single hip prosthesis and with double hip prostheses (see Chapter 8 and 9, respectively). 


\section{Chapter $7 \quad$ CBCT-based dose calculation of a prostate cancer patient}




\subsection{Introduction}

In this chapter, real patient $\mathrm{pCT}$ data was used to test the accuracy of the segmentation of CBCT images. Compared with the previous chapter, a more complex treatment plan, IMRT, was calculated on the processed images and the resultant dose distribution was analysed and compared with the pCT plan. As the prostate is close to the rectum and bladder, IMRT is particularly useful for prostate irradiation (Martin et al., 2010). It has been shown that IMRT increases the ratio of TCP to NTCP of the rectum in the treatment of prostate cancer (De Meerleer et al., 2000). In such a technique, it is assumed that the target position and patient shape do not change during the treatment course. The movement of the prostate is unpredictable and very common, mainly due to changes in rectal volume or bladder filling (see Section 2.2). Such movement increases the risk of a geographical miss and may decrease the dose to the target with increasing dose to OAR, leading to low biochemical control and local control, and increased rates of especially late rectal toxicity. This movement can be corrected by using CBCT imaging. However, the IMRT plans for prostate cancer only deliver doses of 74-78 Gy in 37-39 fractions (2 Gy/fraction). It has been demonstrated that delivering higher doses per fraction may be beneficial for prostate cancer. Dearnaley et al (2015) studied a large UK led trial (CHHiP). The results showed that 60 Gy in 20 fractions (3 Gy/fraction) provides low toxicity rates with equivalent effectiveness and less cost (Dearnaley et al., 2015). In this chapter, this hypofractionation technique was used.

\subsection{Methodology}

\subsubsection{CT and CBCT image acquisition}

A prostate cancer patient was scanned using the same CT scanner used in the previous chapter (see Section 6.2.2). The CT images were acquired with $120 \mathrm{kVp}$ and the tube current was modulated during the scan based on patient anatomy. The matrix size of the reconstructed images was $512 \times 512$ and voxel sizes were $1.19 \times 1.19 \times 3 \mathrm{~mm}^{3}$.

A CBCT scan was acquired with a MFOV, collimator M15 and the F1 filter using the following parameters: $120 \mathrm{kVp}, 25 \mathrm{~mA}, 40 \mathrm{~ms}$ per projection and 660 projections. The matrix size of the reconstructed images was $410 \times 410$, the voxel size was $1 \times 1 \times$ 


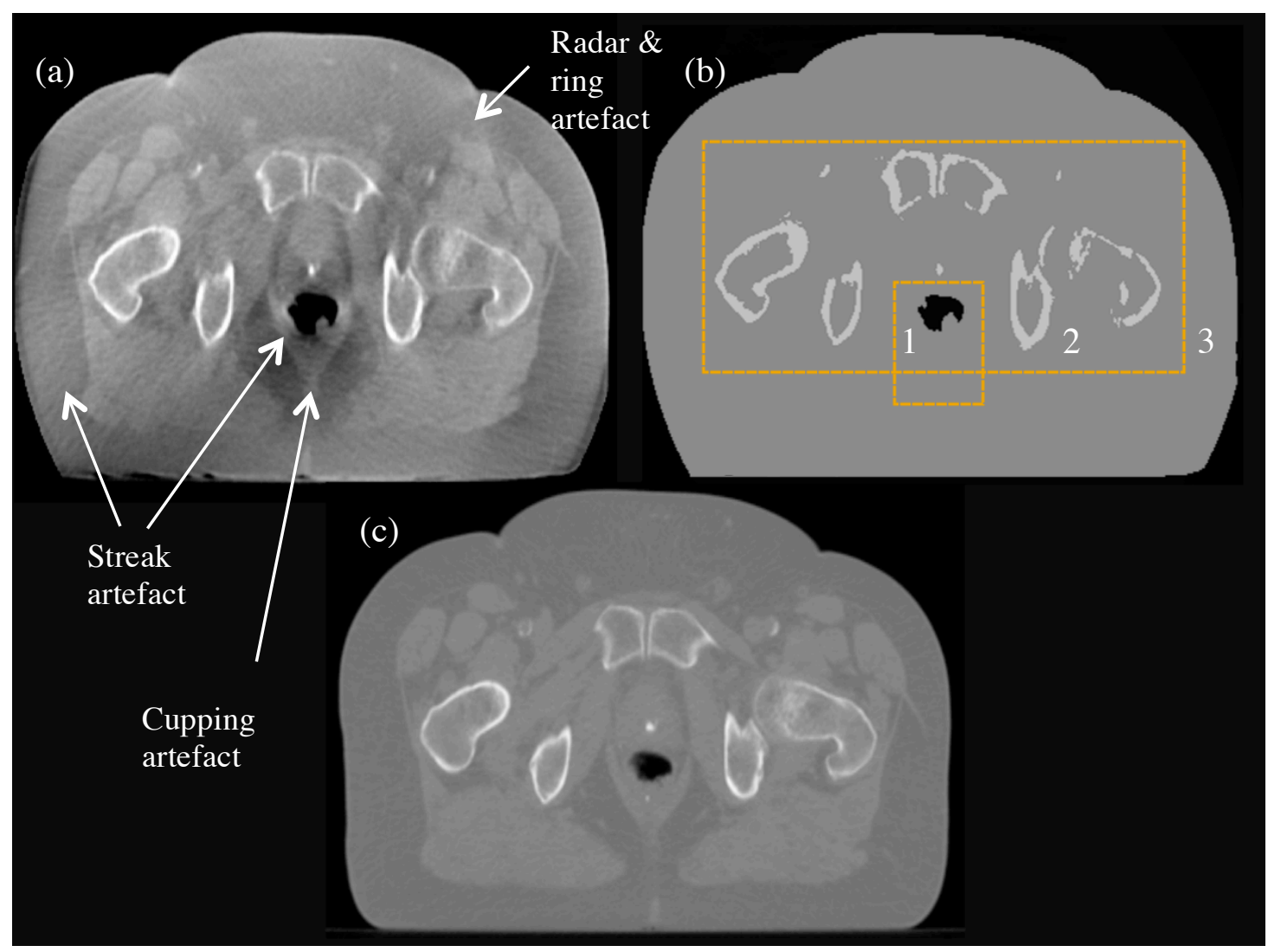

Figure 7.1: A prostate patient scan using (a) CBCT, (c) pCT and the resultant image after segmenting $C B C T$, (b) sCBCT.

$3 \mathrm{~mm}^{3}$ and the scan length in the superior-inferior direction along the phantom (G-T direction) was $16.8 \mathrm{~cm}$.

\subsubsection{Modification of CBCT image}

Figure 7.1 shows the $\mathrm{pCT}, \mathrm{CBCT}$ and $\mathrm{SCBCT}$ images of a prostate cancer patient. The segmentation of CBCT was performed using a similar procedure as in the previous chapter. In this study, besides water $(0 \mathrm{HU})$ and bone $(528 \mathrm{HU})$, an air bin/segment ($976 \mathrm{HU}$ ) was included in the sCBCT images as there was air/gas in the rectum in both scans, pCT and CBCT (Figure 7.1). In contrast to the phantom case, the patient CBCT image set was divided into three regions and each region has individual threshold values for the water, bone and air segments. The division of regions is implemented in the MLT algorithm using the coordinates of each region. The reason for this division was that there were a large number of image artefacts, and more scatter, than in the phantom case, as the patient separation was larger and more inhomogeneous. In addition, the image artefacts, scatter and noise are variant and dependent on the 
position in the CBCT images. Thus the image set was divided into three regions where the variation in the CBCT numbers of a material was small within each region. For example, in region 2 the range of pixel values for bone was $\geq 796$. If this range was used in region 3, it would erroneously replace radar and streak artefacts with the bone CT number (528 HU). The radar artefact is a result of the ghosting effect that is caused by the rapid change in the exposure to the FPD from frame to frame, resulting in incompleteness of projection data (see Section 2.4.7). In the air region, or low density region, this change resulted in an intensity that was higher than in other frames in the projection image, which appeared in the subsequent images, and consequently appeared as a circular bright-shaded region in the reconstructed images. Similarly, if the same range was used in region 1, it would erroneously replace streak artefacts, which appeared in the rectum at the air(gas)-tissue boundaries, with the bone CT number. The streak artefact was caused by gas motion in the rectum that occurred during acquisition of the projection images. Therefore, three threshold values were used for each material in each region, as shown in Table 7.1. If there are misidentified regions, they can be manually and individually corrected by applying the MLT algorithm to subregions of the CBCT (see Figure 6.2). As a result, the segmentation in this way was able to accurately correct for the artefacts in CBCT images, as shown in Figure 7.1(b). The operator time for the segmentation was an hour.

Table 7.1 The threshold values for region 1,2 and 3.

\begin{tabular}{c|ccc}
\multirow{2}{*}{ Region } & \multicolumn{3}{|c}{ Pixel value range } \\
\cline { 2 - 4 } & Air & Water & Bone \\
\hline $\mathbf{1}$ & 0 to 360 & 361 to max & 796 to max \\
\hline $\mathbf{2}$ & - & 0 to 795 & - \\
\hline $\mathbf{3}$ & 0 to 400 & 401 to max & \\
\hline
\end{tabular}

\subsubsection{OMP-based treatment plan}

Compared with a conventional plan, the implementation of IMRT allows the delivery of a highly conformal dose distribution with high precision by modulating both the number of fields and the intensity of radiation within the radiation field using MLCs. 
IMRT uses an inverse planning technique where the dose objectives are set and the TPS calculation optimized to achieve these objectives by making use of DVHs.

Table 7.2: Summary of the prostate treatment plan settings for IMRT.

\begin{tabular}{cccccc} 
& \multicolumn{5}{c}{ IMRT plan } \\
\cline { 2 - 6 } & $\begin{array}{c}\text { No of } \\
\text { Segments }\end{array}$ & $\begin{array}{c}\text { Gantry } \\
\text { angle }\end{array}$ & $\begin{array}{c}\text { Collimator } \\
\text { angle }\end{array}$ & SSD & MU \\
\hline BEAM 1 & 5 & 180 & 0 & 88.4 & 71.6 \\
\hline BEAM 2 & 6 & 100 & 350 & 80.2 & 160.1 \\
\hline BEAM 3 & 5 & 35 & 349 & 85.6 & 101.7 \\
\hline BEAM 4 & 6 & 260 & 16 & 79.3 & 148 \\
\hline BEAM 5 & 6 & 325 & 20 & 85.2 & 123.9 \\
\hline
\end{tabular}

Using the inverse planning option in the OMP software, a segmented IMRT plan with five 6-MV photon fields, at gantry angles of $180^{\circ}, 100^{\circ}, 35^{\circ}, 260^{\circ}$ and $325^{\circ}$ was created for the pCT image set (Table 7.2 \& Figure 7.2). An acceptable uniformity for the PTV was achieved by optimizing the plan with a total of 28 segments. The plan was delivered in 20 fractions, each with a radiation absorbed dose of $3 \mathrm{~Gy}$. The $\mathrm{sCBCT}$ and $\mathrm{pCT}$ images were fused with manual rigid registration using ProSoma software (v3.3, MedCom, Germany) and the structure sets were transferred to the sCBCT images without any modification except for the external contour. The plan was then copied to the sCBCT using the same geometry and MU values. The doses were recalculated using $\mathrm{PB}$ and $\mathrm{CC}$ algorithms, to highlight differences in dose calculation in inhomogeneous regions.

The registration is based on the matching of bony anatomy. If there is a rotation or the legs are in a different place, the two image sets cannot be match as rigid registration assumes that the patient is not in the same place but is the same shape. Even though the same immobilisation devices are used in both scans, there will be differences in the soft-tissue between the two scans other than a translational shift e.g. patient weight changes and internal organ motion. These changes are not taken into account by the rigid registration. Therefore, there is an uncertainty associated with the registration process. Currently, only rigid registration is available for clinical 


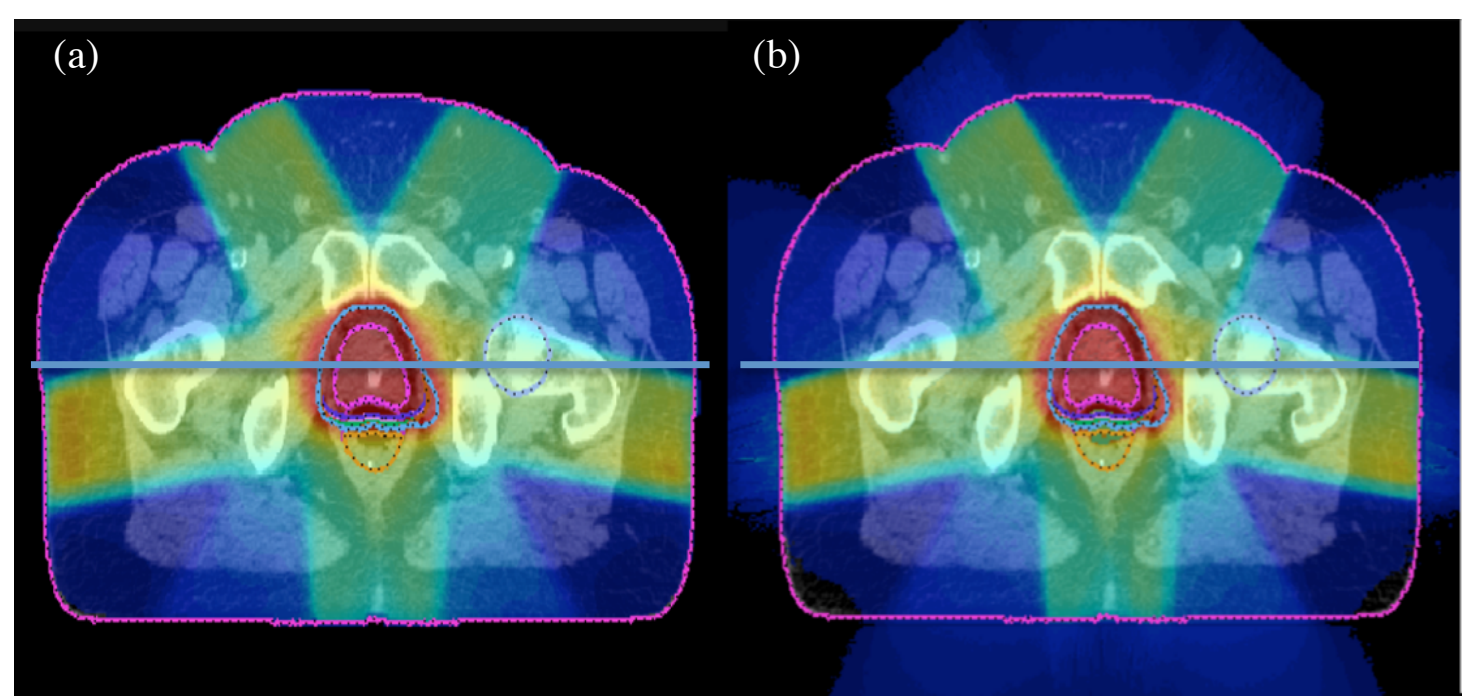

Figure 7.2: A screenshot of a prostate IMRT plan using (a) OMP with CC algorithm and $(b)$ MC algorithm, both displayed using CERR. The horizontal lines show where the cross profiles were taken from.

use. These effects are likely to lead to displacements of less than $3 \mathrm{~mm}$ as discussed in Section 1.3.

\subsubsection{MC-based treatment plan}

The MC plan was performed with the DOSXYZnrc user code using the BEAM treatment head simulation' option as a radiation source. In contrast to the previous chapter where the conventional plan was normalised to give 74 Gy at the isocentre, the IMRT plan was delivered and optimized with no normlisation point. In the OMP system, the plan is based on achieving a prescribed dose to the PTV whilst minimizing the dose to the OARs. The prescribed dose is reported in Gray (Gy). In MC the simulation, the dose is scored in dose per particle, Gy per particle. Therefore this dose needs to be converted into an absolute dose. In the clinic the linacs are calibrated to deliver a set amount of radiation in MUs. The calibration is made by calculating the dose in a water phantom under standard reference conditions so that 1 MU is equivalent to $0.01 \mathrm{~Gy}$ at the depth of maximum dose for a $10 \times 10 \mathrm{~cm}^{2}$ field size at $100 \mathrm{~cm} \mathrm{SSD}$. The calibration factor, $\mathrm{k}$, is then $0.01 \mathrm{~Gy} / \mathrm{MU}$. In the MC simulation, such a calibration factor can be calculated at the same point as the Linac is calibrated by modeling the same conditions using DOSXYZnrc code. The MC calibration factor, $\mathrm{K}_{\mathrm{MC}}$, can be then used to obtain an absolute dose $\left(\mathrm{D}_{\mathrm{abs}}\right)$ using the following equation: 


$$
D_{a b s}=D_{M C}\left(k / K_{M C}\right) U
$$

where $D_{M C}$ is the MC scored dose per particle, $k$ is the Linac calibration factor (Gy/MU) and $U$ is the prescribed MUs (Spezi et al., 2002). $K_{M C}$ is the MC calibration which can be calculated using

$$
K_{M C}=\frac{\overline{D_{M C}}}{\overline{D_{M}}}
$$

where $\overline{D_{M C}}$ and $\overline{D_{M}}$ are the average doses between the depth of $5 \mathrm{~cm}$ and $15 \mathrm{~cm}$ for the MC simulation and the measurement, respectively. The calibration factor, $K_{M C}$, of $9.85 \times 10^{13}$ particle/MU was calculated for the MC model in this thesis using the Elekta Synergy Linac.

For the IMRT plan, a single input file, for each BEAMnrc and DOSXYZnrc, was created for each segment manually based on the treatment plan parameters (Table 7.2). It is time consuming to write an input file for BEAMnrc and another one for DOSXYZnrc, for each segment (total of 28 segments) and these are also subject to random errors. In addition, for the MC sCBCT-based plan, the same BEAMnrc input files of the MC pCT-based plan were used but separate DOSXYZnrc input files were generated as the sCBCT coordinates were different from those in pCT, i.e. plan isocentre. This suggested that an automated procedure should be applied to the DICOM-RT file (both pCT- and sCBCT-based plan file) to extract the treatment plan parameters and then convert and write them in a MC-format input file (egsinp file) for BEAMnrc and DOSXYZnrc, separately (see Chapter 10). The simulation or calculation of each segment was performed on the egsphant file, which was generated from the CT and SCBCT DICOM images of the patient (see Section 6.2.6). Each simulation was run with $90 \times 10^{6}$ histories and split into 100 jobs using 100 nodes, which were run in parallel in HPC-Wales. Then, at the end of the simulation of each segment, the calculated dose distributions from all jobs were combined in a single format file 3ddose. The dose in the 3ddose file of each segment was then weighted by their MUs and converted into an absolute dose using equation 7.1 in a MATLAB script. Finally, the whole IMRT treatment plan was generated by adding all the segments' 3ddose files together. 


\subsubsection{Treatment planning evaluation and comparison}

The $\mathrm{pCT}$ and sCBCT plans were exported from the OMP system as DICOM-RT files that were then imported into CERR. Once the DICOM-RT was imported, the combined MC dose file was imported into MATLAB and then CERR overlaid it on the DICOM images (Figure 7.2). CERR was then used to visualize and evaluate the resultant dose distributions of both the OMP and MC plans.

\subsubsection{DVH comparison}

Cumulative DVHs were generated for the PTV, rectum, bladder and right and left femoral heads and compared between the $\mathrm{pCT}$ and $\mathrm{sCBCT}$ plans. The comparison of DVHs was performed using the OMP PB and CC algorithms, and MC calculation. The Dmax, Dmean and Dmin parameters for the above structures were compared. The coverage of the PTV, the dose to 95\% of the PTV (D95\%), and the volume of PTV with dose $>95 \%$ of the prescription dose (V95), were compared.

\subsubsection{Dose profile comparison}

The dose profiles at a depth of $12.6 \mathrm{~cm}$ were compared and plotted against the CT and sCBCT numbers of the pCT and sCBCT, respectively (Figure 7.2). Such a comparison was made to show the accuracy of segmenting and converting the CBCT pixel values in heterogeneous regions, and what the effect of this conversion was on the dose distribution. The relative difference between pCT and sCBCT dose profiles was plotted to aid comparison.

\subsubsection{Gamma index comparison}

The comparison between $\mathrm{pCT}$ and sCBCT dose distributions at the pixel level was made using gamma evaluation. The $\gamma$ index analysis was performed inside the above structures and the pCT plan was considered as the reference plan. The criteria were $3 \%$ DD, 3 mm DTA and 5\% low dose threshold. 


\subsubsection{NTCP comparison}

One of the most important late toxicities of radiotherapy for prostate cancer is the rectal toxicity and late bleeding. Thus the rectum and other OARs should receive doses less than a constraint dose. This can be achieved with the use of the IMRT technique. However, rectal distension and internal motions increase the risk of a geometrical miss and have a significant impact on excessive normal tissue toxicity in the rectum. Therefore, the NTCP, as a biological endpoint parameter, was calculated for the rectum and compared between $\mathrm{pCT}$ and sCBCT plans. The Lyman-KutcherBurman (LKB) model was used to calculate the NTCP values. The model is initially based on the Lyman model which describes complication probabilities for uniformly irradiated organ volumes (Lyman, 1985). Normal tissues are rarely uniformly irradiated, thus the Lyman model is incomplete. Kutcher and Burman (1989) proposed a DVH reduction algorithm to convert a heterogeneous dose distribution into a uniform organ irradiation using the effective volume method (Kutcher and Burman, 1989). This method is most commonly used to complement the Lyman model and the combination of both is referred to as the LKB model (Mayles, 2007, Li et al., 2012). The LKB model is the most well known and used model to predict the NTCP after a radiotherapy treatment (D'Avino et al., 2015). The NTCP can be calculated using the following equations:

$$
N T C P=\frac{1}{\sqrt{2 \pi}} \int_{-\infty}^{t} e^{\frac{-t^{2}}{2}} d t
$$

where $t$ is defined as

$$
t=\frac{E U D-D_{50}(v)}{m D_{50}(v)}
$$

and

$$
E U D=\left[\frac{1}{N} \sum_{i=1}^{i=N} D_{i}^{1 / n}\right]^{n}
$$

The parameter EUD represents the generalized equivalent uniform dose delivered to the organ, the rectum in this case, that leads to the same NTCP as the actual nonuniform dose distribution. D50 is the tolerance dose to the whole organ which, for 
a given partial volume fraction $\mathrm{v}$, results in a $50 \%$ complication risk, $\mathrm{m}$ is the slope of NTCP (sigmoid curve), $D_{i}$ is the dose at voxel i, N is the total number of voxels and $n$ is the volume effect parameter which can assume values in the range 0-1 (Mohan et al., 1992, Li et al., 2012, Bansal et al., 2012).

From the literature, three sets of LKB parameters were chosen to minimize any impact of the LKB model parameters. This was suggested by Hardcastle et al (2011), where the three sets were taken from Tucker et al (2004), Rancati et al (2004) and Sohn et al (2007). This study uses parameters from the last two studies and a more recent parameter set proposed by Michalski et al (2010). The reason why the Tucker et al (2004) parameter set was excluded is that the volume parameter, $n$, has a large value (1.03) which corresponds to a large volume effect and the architecture has more parallel tissue. The three chosen sets of LKB parameters used in this study have small $\mathrm{n}$ values which correspond to small volume effects (series architecture), indicating that high dose regions play an important role in determining the risk of late rectal toxicity. Based on the literature, the $\mathrm{n}$ value usually is quite small $(<0.15)$ (Michalski et al., 2010). The three chosen sets of LKB model parameters are given in Table 7.3, all representing $\geq$ Grade 2 rectal toxicity. The CERR toolkit was used to calculate the NTCP values.

Table 7.3: NTCP calculation parameters.

\begin{tabular}{ccccc} 
& \multicolumn{4}{c}{ Parameter Set } \\
\cline { 2 - 5 } & $\mathbf{n}$ & $\mathbf{m}$ & D50 & Reference \\
\hline NTCP1 & 0.24 & 0.14 & 75.7 & Rancati et at (2004) \\
\hline NTCP2 & 0.084 & 0.108 & 78.4 & Sohn et al (2007) \\
\hline NTCP3 & 0.09 & 0.13 & 76.9 & Michalski et al (2010) \\
\hline
\end{tabular}

\subsection{Results and discussion}

\subsubsection{DVH comparison}

Figure 7.3 shows the DVH of the prostate IMRT plan with a prescription dose of 60 

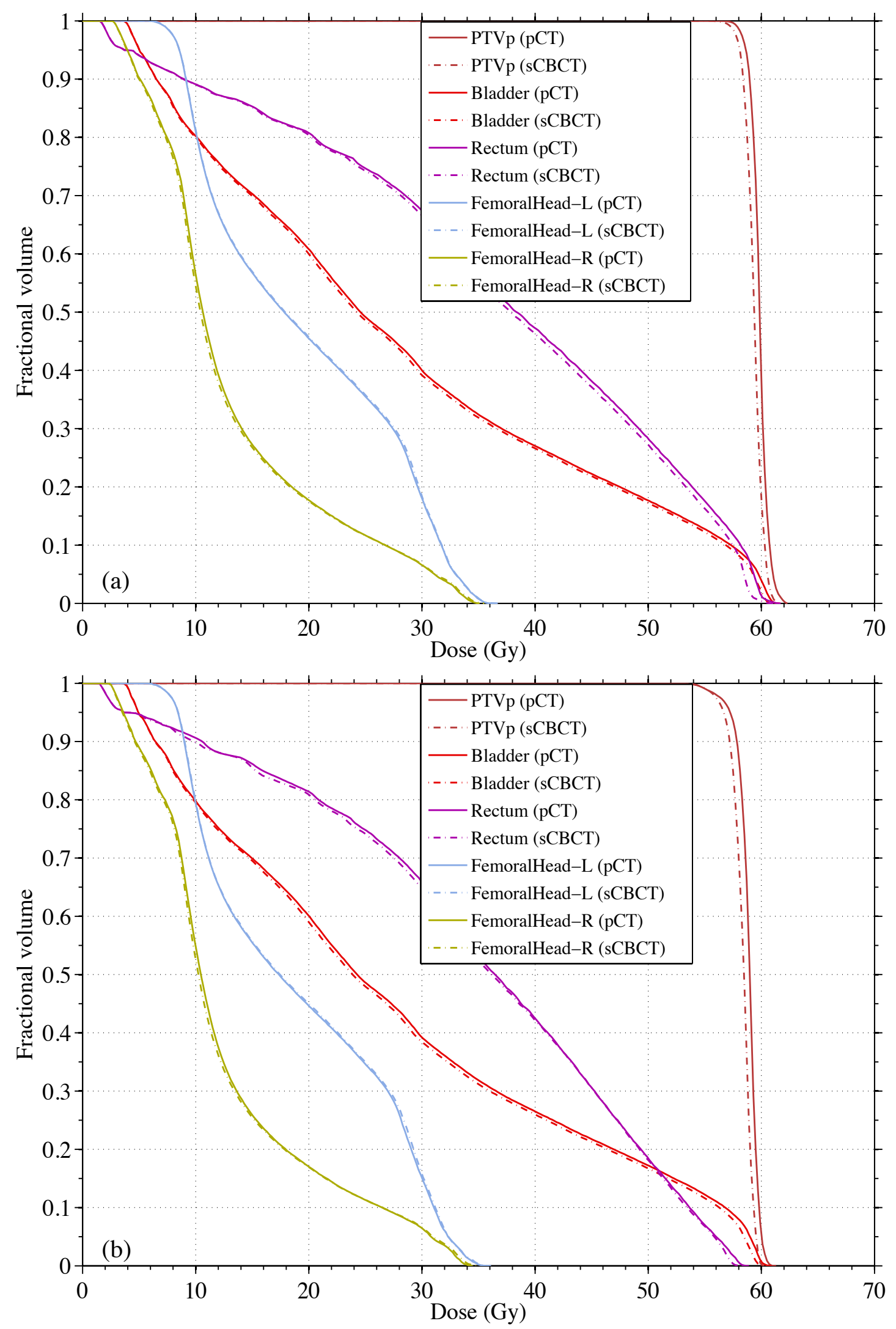


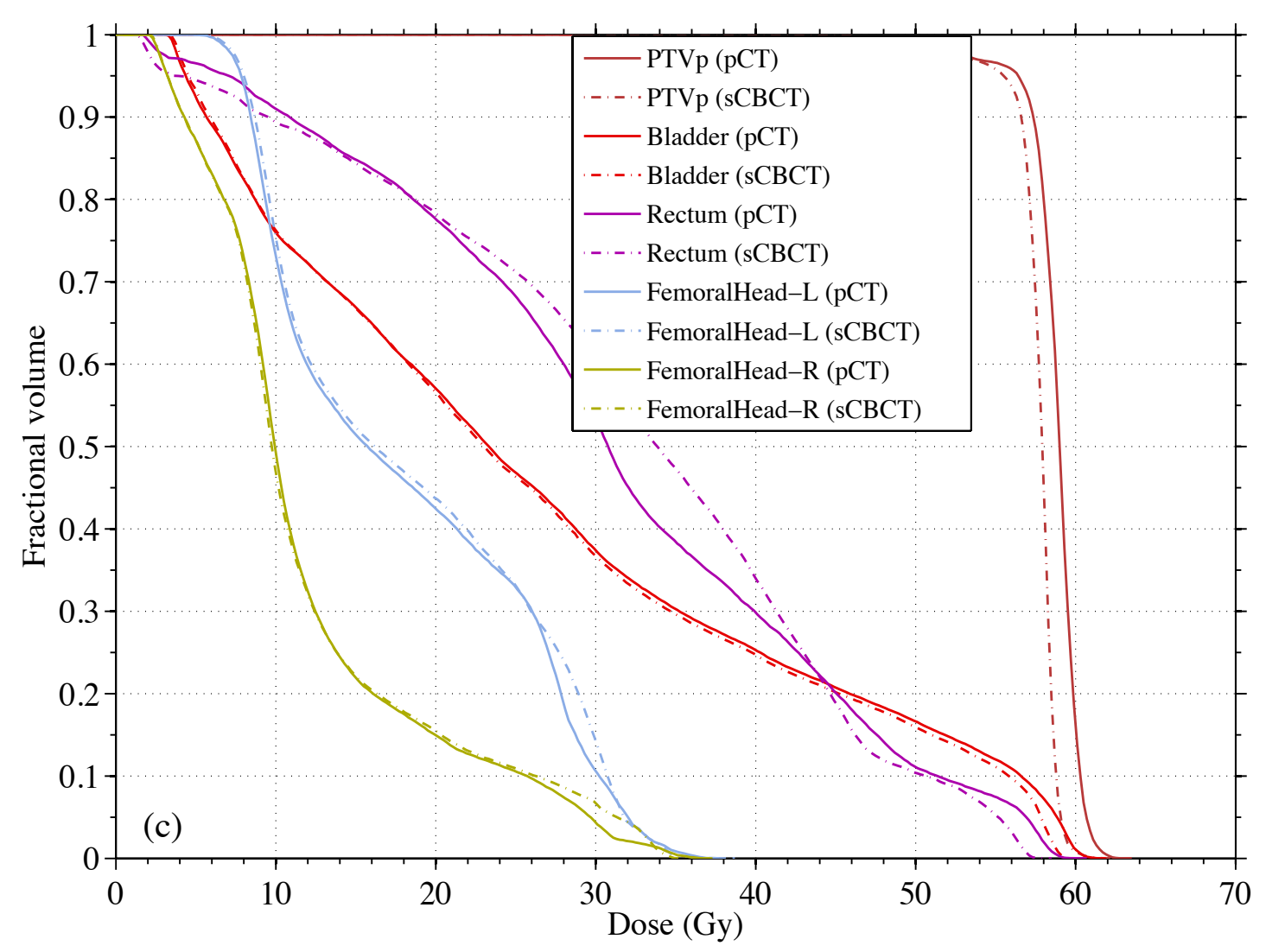

Figure 7.3: DVHs comparison between $p C T$ and sCBCT plans for PTV, rectum and bladder using (a) $P B$, (b) $C C$ and (c) MC algorithm (prescription dose $60 \mathrm{~Gy}$ ).

Gy in 20 fractions. It shows the dose of the pCT and sCBCT plans to the PTV, rectum, bladder, right and left femoral heads using the (a) PB, (b) CC and (c) MC algorithms.

It can be clearly seen that the sCBCT plan is in a good agreement with the pCT plan. In general, the SCBCT plan slightly underestimated the dose to all the structures, except the left and right femoral head structures when using (a) PB and (b) CC algorithms. The sCBCT plan overestimated the left femoral head minimum dose by $3.38 \%$ and $3.51 \%$ when using PB and CC algorithms, respectively (Table 7.3). The right femoral head maximum dose was overestimated by the SCBCT plan by $1.13 \%$ and $1.15 \%$, when using PB and CC algorithms, respectively. This indicated that the left and right femoral heads volumes were underestimated in the SCBCT images and considered as water, thus the doses were higher in these regions. For the MC algorithm, the largest difference was found in the PTV minimum dose where it was overestimated by $61.64 \%$ in the sCBCT plan. This may be due to the fact that the air/gas pocket volume in the rectum in the SCBCT was less than in the pCT. 
Table 7.4: Dose and coverage differences between sCBCT and pCT plans, in \%, for the PTV, rectum, bladder and left and right femoral heads.

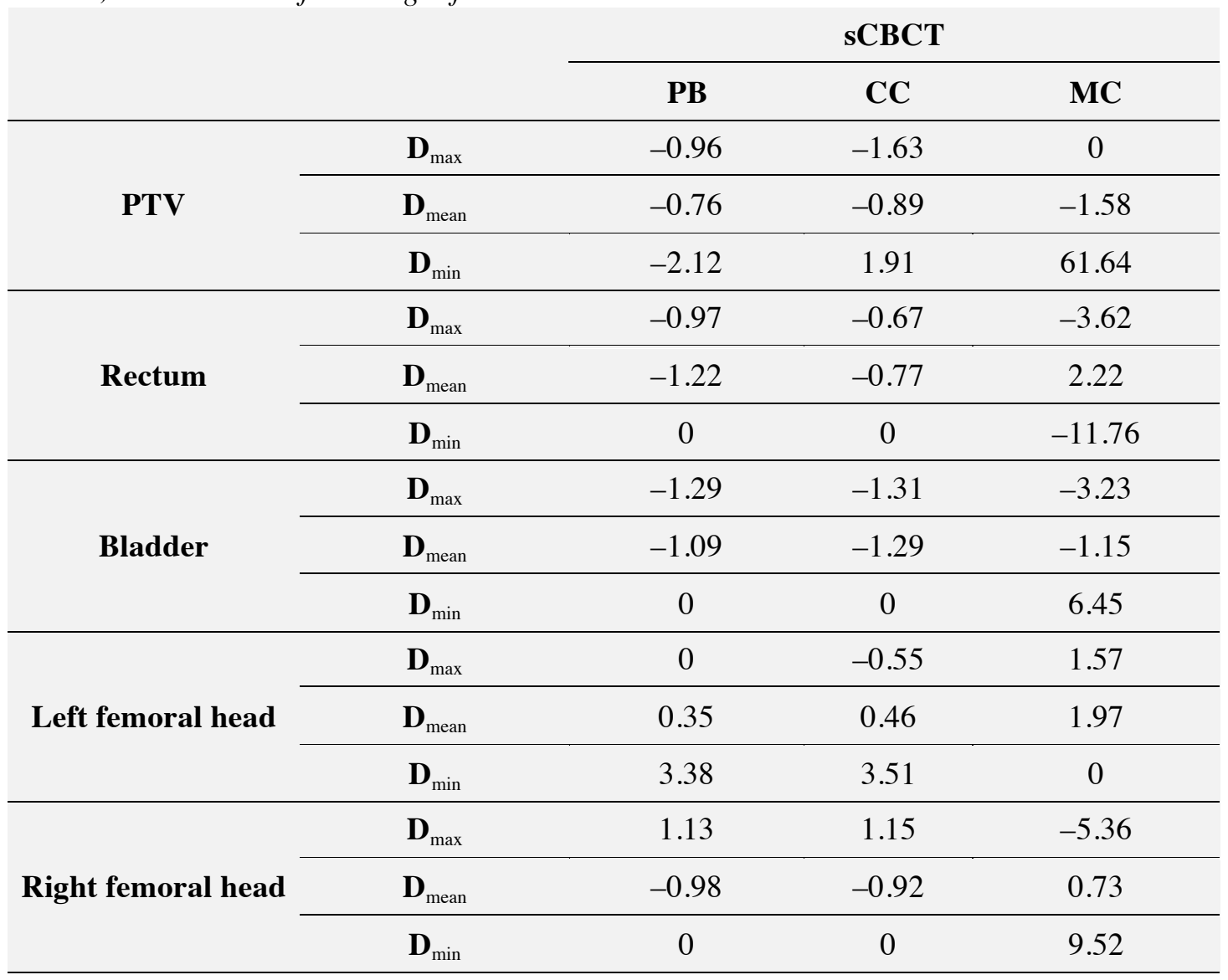

Consequently the anterior rectal wall was partially inside the PTV and the MC algorithm was sensitive to such a heterogeneous region, thus, giving lower doses in that region of the pCT. This is due to the difference in density between (of several magnitudes) air and water, leading to a significant difference in the mass-stopping powers (Knöös et al., 2006). The CC algorithm also overestimated the PTV minimum dose by $1.91 \%$, whilst the PB algorithm underestimated the PTV minimum dose by $2.12 \%$. This difference means that the segmentation of CBCT images using the MLT algorithm underestimated the air/gas pocket volume. The volume difference was found to be $-12.5 \%$. This may be due to the threshold method, where the partial volume in the rectum was considered either air or water based on the threshold values.

Another reason for the underestimation in volume in the sCBCT may be due to a real difference in the rectum volume between the two scans. This volume difference resulted in higher volumes, in the rectum, receiving doses over the range of 0-45 Gy in the sCBCT plan compared with the $\mathrm{pCT}$ plan when using the $\mathrm{MC}$ algorithm. The 
difference in the rectum mean dose was $-1.22 \%,-0.77 \%$ and $2.22 \%$ when using PB, $\mathrm{CC}$ and MC algorithms, respectively. This finding is similar to the Onozato et al (2014) study. This study used the MLT algorithm to segment the CBCT into air, fat, muscle and bone and found that the average difference in the rectum Dmean was less than $1.2 \%$ when using the AAA algorithm (Onozato et al., 2014). The results also showed that the average difference in Dmean for PTV between the pCT and the modified CBCT was less than $0.65 \%$.

Table 7.5 shows the PTV coverage data. In general, the sCBCT plan slightly underestimated the dose to the PTV compared with the pCT plan. The sCBCT plan resulted in a lower dose to $95 \%$ of the PTV, by $-0.8 \%,-1 \%$ and $-1.5 \%$ when using $\mathrm{PB}, \mathrm{CC}$ and $\mathrm{MC}$ algorithms, respectively. The difference in the V95\% parameter was $-0.5 \%$ when using the $\mathrm{PB}$ algorithm whilst the $\mathrm{CC}$ and $\mathrm{MC}$ algorithms showed differences of $-3.7 \%$ and -10.3 respectively. This indicated that the SCBCT numbers were higher in the PTV region, representing only water with a value of 1024 , compared with the pCT numbers, which represent a range of soft tissue that include low pCT numbers for adipose tissues (with a value $<928$ ). Such differences were not picked by the $\mathrm{PB}$ algorithm whilst the $\mathrm{CC}$ and $\mathrm{MC}$ algorithms showed larger dose differences.

Table 7.5: PTV coverage for the pCT and sCBCT plans. The dose to 95\% of PTV volume, $D 95 \%$, and the volume receiving $95 \%$ of prescription dose.

\begin{tabular}{ccc|cc} 
& \multicolumn{2}{c}{ pCT } & \multicolumn{2}{c}{ SCBCT } \\
\cline { 2 - 5 } & D95\% & V95\% & D95\% & V95\% \\
\hline PB & 58.6 & 99.9 & 58.1 & 99.4 \\
\hline CC & 57.2 & 96 & 56.6 & 92.4 \\
\hline MC & 56.4 & 92.6 & 55.5 & 83 \\
\hline
\end{tabular}

\subsubsection{Dose profile comparison}

Figure 7.4 shows the cross-profiles of the pCT and SCBCT plans at a depth of 12.6 $\mathrm{cm}$, as well as the pCT numbers and sCBCT numbers at that depth, using PB, CC and MC algorithms, respectively. Each has a subplot that aids a clear comparison between the $\mathrm{pCT}$ and sCBCT dose profiles. In general, the sCBCT profiles are in good 

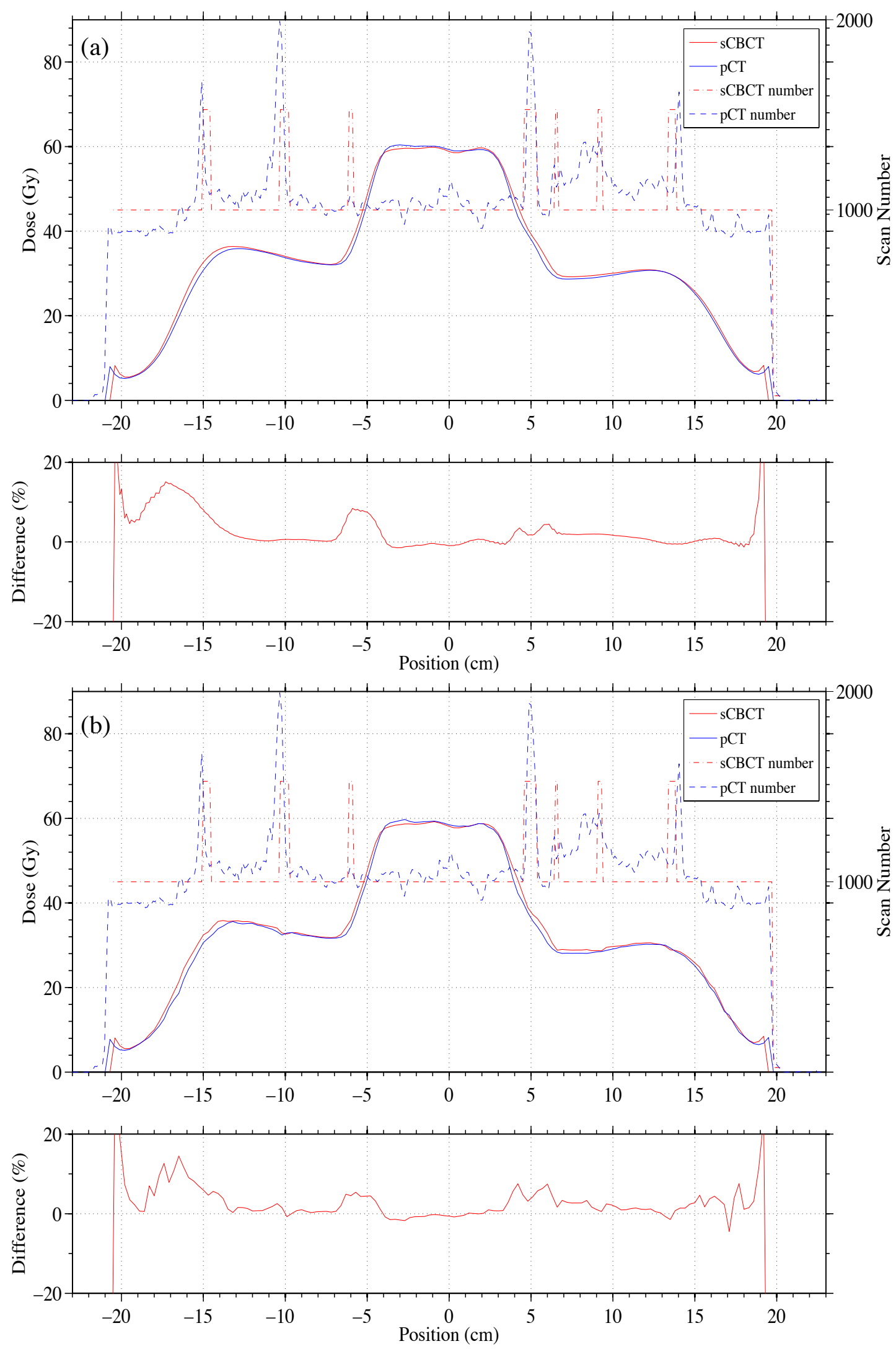

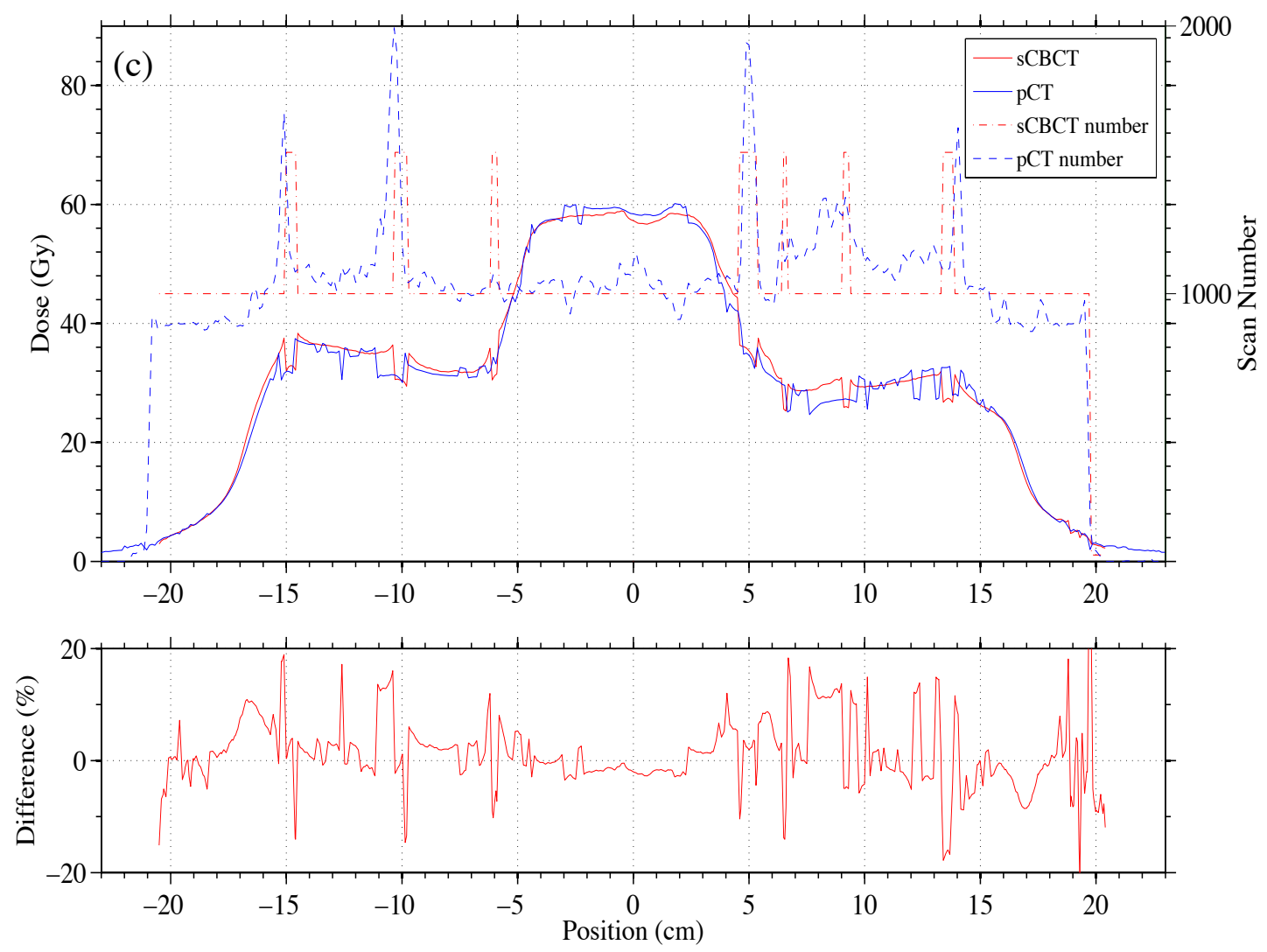

Figure 7.4: Comparison of the dose profile of $p C T$ and $s C B C T$ plans at the isocentre depth using (a) $P B$, (b) $C C$ and (c) MC algorithm. The second $y$ axis represents the $S C B C T$ number and $C T$ number. Subplots represent the differences between $p C T$ and $S C B C T$ dose profiles.

agreement with the pCT profiles when using all algorithms. In high density regions, where the pCT images showed high pCT numbers, the sCBCT numbers quite accurately represent these regions with small changes. For example, in the right side of the plots, the sCBCT numbers underestimated some high density regions and considered it as water, whereas the pCT numbers increased gradually with some fluctuations. Thus, compared with the pCT dose profile, the sCBCT doses were higher in these regions and were lower in the periphery regions as the sCBCT numbers overestimated those regions, which were adipose tissues represented by the pCT numbers. This suggested that the adipose tissues HU or CT number (bin) should be included in the segmentation of the CBCT images (see Chapter 8). However, the three bins were enough to provide a clinically acceptable dose accuracy.

The MC algorithm showed strong sensitivity to any change in the CT or sCBCT numbers and the $\mathrm{CC}$ algorithm was also sensitive especially in the high density region, as shown in Figure 7.4(b) and 7.4(c), respectively. On the other hand, the PB 
algorithm was the least sensitive to the change in the CT and SCBCT numbers. The PB algorithm in OMP calculates dose to water, whilst the CC algorithm calculates dose to medium, as does the MC algorithm (Knöös et al., 2006). Thus it is expected that $\mathrm{PB}$ would provide higher doses than $\mathrm{CC}$ and $\mathrm{MC}$ by 1-2\% (depending on homogeneities), due to the differences in mass energy absorption coefficients that depend on atomic composition. Using the PB algorithm, the density variation in a heterogeneous medium is accounted for by the EPL method for the primary dose (see Chapter 3). For the scatter dose (including secondary electrons), the dose is calculated using the geometrical depth and it is then multiplied by a 1D convolution correction factor, which is calculated using the radiological depth to account for heterogeneities along the pencil beam (Ahnesjö et al., 1992). Thus the PB algorithm cannot accurately model the scatter and electron transport in lower or higher (than water) density mediums where electronic disequilibrium occurs. Thus the PB algorithm underestimates the lateral scattered dose and overestimates the dose at the calculation point (in the beam direction), as shown in Figure 7.4.

In the $\mathrm{CC}$ algorithm, the density variation is accounted for by applying a density scaling that relies on rectilinear scaling based on O'Connor's theorem. This is applied directly to the point kernels, which are convolved with TERMA. Thus the kernels represent the energy from primary electrons and scattered photons (Ahnesjö, 1989). For doses due to electrons generated by primary photons, the rectilinear scaling is valid but for subsequently scattered photons and their recoil electrons the rectilinear approximation is no longer valid. Therefore, the $\mathrm{CC}$ algorithm is an approximation for multiple scatter (Krieger and Sauer, 2005).

In the MC algorithm, the primary, scatter components and electron transports, are accounted for explicitly, if the energy transfer during interactions is above an arbitrary energy threshold. However, there are approximation methods applied to increase the simulation efficiency (see Chapter 4 and 5). Particles, with energy below the threshold, are terminated and the energy deposited locally. Since MC and CC algorithms calculate dose to medium, the $\mathrm{HU}$ or $\mathrm{CT} / \mathrm{sCBCT}$ numbers of the medium must be provided precisely. Therefore, the PB algorithm would be less sensitive than $\mathrm{CC}$ and $\mathrm{MC}$ for calculating the dose using different scans. Thus MC and CC algorithms minimised uncertainty related to the dose calculation and also identified 
those introduced by different scans. However, the relative dose difference at the centre of the PTV was $-0.9 \%,-0.5 \%$ and $-1.5 \%$ when using PB, CC and MC algorithms, respectively. Such differences are considered to be clinically acceptable.

Besides any internal differences between the two scans, the lateral (x,y) FOV in some of the CBCT images was cropped due to the combination of the medium FOV and a large scanned patient, as shown in the sCBCT number profile. It has been suggested that relative information from pCT could substitute the missing volumes (Lee et al., 2008a). Alternatively, the missing area can be similarly substituted with water-equivalent tissue, based on the outline of the pCT images (van Zijtveld et al., 2007, Fotina et al., 2012). These corrections were not used in this study because the missing volume was never more than $5 \mathrm{~mm}$, as guided by pCT images and the contribution of a single beam was to increase the dose by at most $0.4 \%$. It was also not situated on the central axis of any beam and therefore did not affect the dose calculated at the PTV.

The FOV can be changed from MFOV to LFOV to cover the whole patient, where FPD can be shifted laterally by $19 \mathrm{~cm}$ from the central axis by using the L collimation. However, this mode significantly degrades the image quality and increases the overall radiation dose to the patient. Currently, as a compromise between image quality, radiation dose and FOV, the $\mathrm{M}$ collimation is used in Singleton's clinical protocol for pelvis patients.

\subsubsection{Gamma index comparison}

Table 7.6 shows the $\gamma$ index evaluation for the calculation points falling inside the PTV, rectum, bladder and right and left femoral heads for the sCBCT plan using PB, $\mathrm{CC}$ and $\mathrm{MC}$ algorithms. The results are shown as the percentage of calculation points resulting in $\gamma \leq 1$. As a result, almost all calculation points passed the $3 \% / 3 \mathrm{~mm}$ criteria when using PB and CC algorithms. However, the MC algorithm showed more calculation points failed the test compared with the other algorithms, but still more than $97.31 \%$ passed the test in the rectum. This is in agreement with previous studies (Boggula et al., 2009). The results also showed that the femoral heads were accurately represented in the sCBCT images. The segmentation method used in this study was accurate enough to separate high density regions from low density regions, even with 
the presence of image artefacts. Thus it shows that using three material bins is accurate to segment $\mathrm{CBCT}$ images of a typical prostate patient (without any prosthesis).

However, as expected, the PB algorithm failed to detect the volume difference in the rectum and that was caused by either the segmentation procedure or by internal organ motion. The CC and MC algorithms resulted in $99.45 \%$ and $97.31 \%$, respectively, of the calculation points in the rectum passing the test. When using the MC algorithm, there were a high number of failure points in the rectum and this shows that there was a difference in the rectum volume between the two scans. This was caused by either the segmentation method or by the internal organ motion. However, such a difference is considered to be clinically acceptable. It was stated that the $\gamma$ value is considered acceptable when the passing rate is greater than $95 \%$ with $3 \mathrm{~mm}$ DTA and $3 \%$ DD criteria (Son et al., 2015).

Table 7.6: Summary of the $\gamma$ index with fixed DTA $=3 \mathrm{~mm}$ and $D D=3 \%$ for the calculation points falling inside the PTV, rectum, bladder and right and left femoral heads showing the percentage of points resulting with $\gamma \leq 1$.

\begin{tabular}{cccc} 
& \multicolumn{3}{c}{ sCBCT } \\
\cline { 2 - 4 } & PB & CC & MC \\
\hline PTV & 100 & 100 & 99.44 \\
\hline Rectum & 100 & 99.45 & 97.31 \\
\hline Bladder & 100 & 100 & 99.97 \\
\hline Left femoral head & 100 & 100 & 99.77 \\
\hline Right femoral head & 100 & 100 & 99.91 \\
\hline
\end{tabular}

\subsubsection{NTCP comparison}

Since there was a significant difference in the rectum volume between the pCT and sCBCT scans, the NTCP was calculated for the rectum using the LKB model with three sets of LKB model parameters (see Table 7.2). The lower the NTCP, the better the plan and is considered to be superior. Figure 7.5 shows the calculated NTCP for the IMRT plan based on pCT and sCBCT images when using (a) PB, (b) CC and (c) MC algorithms. The rectal cumulative DVH was reduced to a single representative 

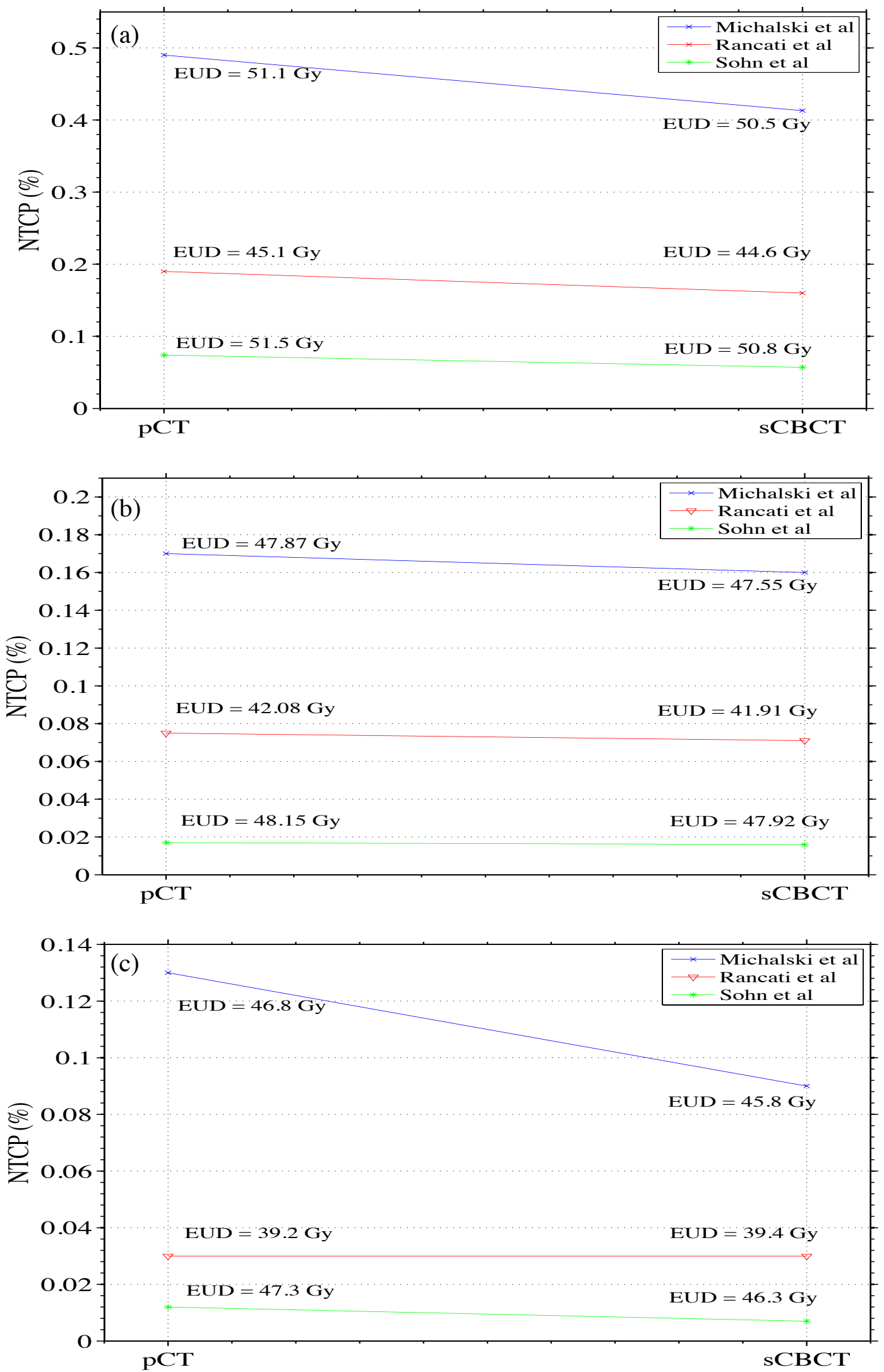

Figure 7.5: NTCPs for IMRT plan based on pCT and sCBCT when using (a) PB, (b) CC and (c) MC algorithms. 
measure, EUD, which is then converted into a probability of complication using a mathematical model based on estimated parameters.

For all parameter sets, NTCP1, NTCP2 and NTCP3, the sCBCT plan resulted in lower NTCPs, and similar EUDs, compared with the pCT plan, when using all algorithms. This may be due to the larger rectum volume in the pCT images than in the sCBCT images, where the volume difference was $12 \%$. This leads to a higher probability of rectal complication in the pCT based IMRT plan. Therefore, the segmentation of CBCT using the method in this study did not affect the IMRT plan goals and achieved similar NTCPs in the rectum.

\subsection{Conclusion}

The CBCT images can be used for dose calculation if the pixel values, or CBCT numbers were corrected (using the MLT method) to represent CT numbers or HUs that are similar to those in the pCT images used for planning. In this chapter, it has been demonstrated that the segmentation of CBCT images into three materials, mainly air, water and bone, using the MLT algorithm, provides accurate dose calculations with differences of less than $2 \%$. Similar dose distributions to the original treatment plan were found when dose calculations were performed on the MLT corrected CBCT images (sCBCT). Gamma evaluation showed that more than more than $97 \%$ of the calculation points passed the test. Using the method in this study, the CBCT image artefacts, such as streak, cupping, ring and radar artefacts, were corrected and accurately replaced with the correct CT numbers. If a patient losses weight or their tumour changes size, performing dose calculation on SCBCT would save time and more importantly reduce radiation dose as it eliminates re-scanning on fan beam CT. Thus it can be used as a quick-decision-making tool for re-planning. The next step is to optimize the number of CT bins for more challenging circumstances as for a prostate patient with single hip prosthesis. 
Chapter 8 CBCT-based dose calculation of a prostate cancer patient with single hip prosthesis 


\subsection{Introduction}

In the previous chapter, the segmentation of CBCT images of a prostate patient into three bins, air, water and bone, using the MLT algorithm was presented. The result showed that the three bins were enough to improve the dose calculation accuracy. The results also showed that the normal tissue complication probability for OARs was maintained at low levels as intended by the original plan. The occurrence of inhomgeneities in the patient anatomy, e.g. metallic hip replacement, has the ability to complicate the segmentation process. The amount of scatter increases with the presence of a metallic hip as the high attenuation material results in more image artefacts. The image quality degrades significantly because of the artefacts caused by the metal, which is a combination of different effects. These include scatter, beam hardening, quantum noise, zero data and photon starvation. These effects result in dark and bright streaks, cupping, ring and radar artefacts that lead to a large variation in the CBCT reconstructed pixel values (see Section 2.5.4). Such a circumstance makes the correction of CBCT data more challenging. The presence of metal also degrades the pCT image quality. A study of the literature showed that no studies of CBCT corrections for dose calculation has looked at a patient with prostheses. In this chapter, CBCT images of a prostate cancer patient with a metallic prosthetic right hip replacement was used, which would provide a more challenging assessment of dose calculation using CBCT. A larger number of material bins (up to 8) than typically used in previous works was explored to find an optimum solution, balancing, segmentation speed with dose calculation accuracy as well as volume consistency between pCT and CBCT images of stable volumes. The main aim of this chapter was to find how many material bins were needed to improve the dose accuracy in such a case with reasonable operator time.

\subsection{Methodology}

\subsubsection{CT and CBCT image acquisition}

A prostate cancer patient with a metallic prosthetic hip was scanned using the same CT scanner used in the previous chapters (see Section 6.2.2). The pCT images were acquired with $120 \mathrm{kVp}$ and the tube current was modulated during the scan based on patient anatomy. The matrix size of the reconstructed images was $512 \times 512$ and 
voxel sizes were $1.06 \times 1.06 \times 3 \mathrm{~mm}^{3}$. The scan length in the superior-inferior direction was $23.7 \mathrm{~cm}$. A CBCT scan was acquired with a MFOV, and collimator M10 and the F1 filter using the following parameters: $120 \mathrm{kVp}, 32 \mathrm{~mA}, 40 \mathrm{~ms}$ per projection and 660 projections. The matrix size of the reconstructed images was $410 \times$ 410 and the voxel size was $1 \times 1 \times 3 \mathrm{~mm}^{3}$ and the scan length in the superior-inferior direction along the phantom (G-T direction) was $12 \mathrm{~cm}$.

\subsubsection{Modification of CBCT image}

The MLT algorithm involves categorising pixel values in the CBCT images into segments of homogeneous $\mathrm{HU}$ using MATLAB scripts to generate sCBCT. In the phantom and patient case, in Chapter 6 and 7, the dose accuracy was improved by segmenting the CBCT into two and three material bins, respectively. In this chapter, the hip prosthesis, and the range of soft tissue types and different materials densities suggested that more bins would be needed to take into account this variation. The maximum number of material bins used in this study was eight bins (sCBCT8) that represent air (-976 HU), two adipose tissues (-135 and -96 HU), water (0 HU), muscle (48 HU), soft bone (200 HU), hard bone (528 HU) and metal implants (2976 HU) (see Table 8.1). The soft bone bin was then excluded and considered as hard bone in SCBCT7, and in SCBCT6 the muscle bin was excluded and considered to be water. In sCBCT5, the adipose1 bin was excluded and considered as adipose2, whilst sCBCT4 excluded both adipose bins. The minimum number of bins used was three bins (sCBCT3) that represent air, water and hard bone, which are the main materials in the pelvis region, as used in Chapter 7. The combination of different bins in each sCBCT was chosen to create a realistic balance between the main three materials. In general, the range of pixel values in the CBCT images were: air (0 to 200), adipose tissues (201 to 600 and 601 to 700 ), water (701 to 800 ), muscle (801 to 875), soft bone (876 to 1000 ), hard bone (1001 to 1600$)$ and metal implant (1601 to 8000).

For the patient case, in Chapter 7, the CBCT image set was divided into three different regions, where the variation in the CBCT numbers of a material is small in each region. Each region has individual threshold values for the air, water and bone segments. Such a method was sufficiently accurate to correct the CBCT artefacts. For the patient with a hip prosthesis case, the threshold values change geometrically since 
noise and scatter in CBCT is variable and dependent on position in the image, especially in the presence of high density materials (Pineda et al., 2008).

Figure 8.1( $\mathrm{a}$ and $\mathrm{c}$ ) shows two different slices and different locations of the original CBCT images. Figure 8.1(b) shows the segmentation of the pixel values using specific threshold values, which can correct ring and radar artefacts. These values are valid even in high density regions. On the other hand, in the presence of the hip prosthesis (higher density region), these threshold values are no longer valid and would overestimate the $\mathrm{HU}$ values in that region due to the increased amount of X-ray scatter and beam hardening resulting in different forms of artefact, such as cupping or dark shading, streaks, ring and radar artefacts, as shown in Figure 8.1(d) (see Section 2.5.4). The artefacts affect the pixel values and therefore affect the segmentation compared with the pCT image (Figure. 8.1(e)). This means that the CBCT data should be divided into regions with sets of different threshold values, which are determined on a region-by-region basis, and should be applied to these regions to accurately correct for the artefacts, as shown in Figure 8.1(f). In general, the greater the variation in the scatter the greater the number of regions that need to be considered and the size of the region decreases as it gets closer to inhomogeneities. Thus, threshold values were highly variable across the whole CBCT data set in this study, particularly in the presence of the hip prosthesis. If there are misidentified regions, they can be manually and individually corrected by applying the MLT algorithm to subregions of the CBCT (see Figure 6.2).

Table 8.1: Number of bins used in sCBCT image sets.

\begin{tabular}{ccccccc} 
& \multicolumn{7}{c}{ Bins } \\
\cline { 2 - 7 } Materials & sCBCT3 & sCBCT4 & sCBCT5 & sCBCT6 & sCBCT7 & sCBCT8 \\
\hline Air & $\checkmark$ & $\checkmark$ & $\checkmark$ & $\checkmark$ & $\checkmark$ & $\checkmark$ \\
\hline Adipose1 & $x$ & $x$ & $x$ & $\checkmark$ & $\checkmark$ & $\checkmark$ \\
\hline Adipose2 & $x$ & $x$ & $\checkmark$ & $\checkmark$ & $\checkmark$ & $\checkmark$ \\
\hline Water & $\checkmark$ & $\checkmark$ & $\checkmark$ & $\checkmark$ & $\checkmark$ & $\checkmark$ \\
\hline Muscle & $x$ & $x$ & $x$ & $x$ & $\checkmark$ & $\checkmark$ \\
\hline Soft bone & $x$ & $x$ & $x$ & $x$ & $x$ & $\checkmark$ \\
\hline Hard bone & $\checkmark$ & $\checkmark$ & $\checkmark$ & $\checkmark$ & $\checkmark$ & $\checkmark$ \\
\hline Titanium alloy & $x$ & $\checkmark$ & $\checkmark$ & $\checkmark$ & $\checkmark$ & $\checkmark$ \\
\hline
\end{tabular}




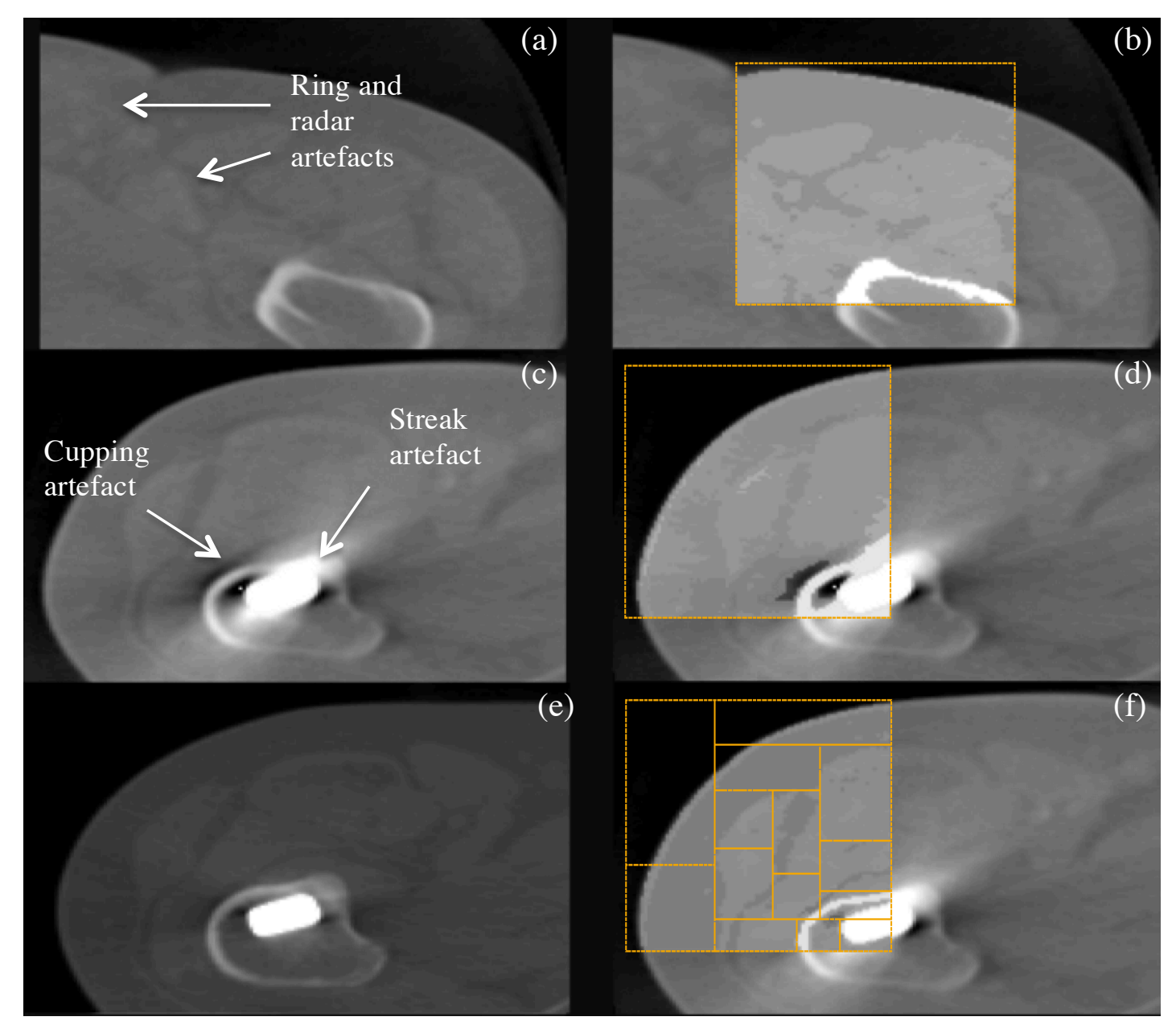

Figure 8.1: Two different locations and slices of the original CBCT ( $a$ and $c$ ) and the resultant images after segmentation using the same threshold values in only a part of the image ( $b$ and $d$ respectively). In particular it is observed in $(d)$ that the artefacts in the vicinity of the metal are erroneously corrected. Variable threshold values were then used $(f)$ which compared well with the original pCT image (e).

\subsubsection{OMP-based treatment plan}

Using the inverse planning option in OMP software, a segmented IMRT plan with five 6-MV photon fields, at gantry angles of $180^{\circ}, 110^{\circ}, 65^{\circ}, 225^{\circ}$ and $325^{\circ}$ was calculated on the pCT image set (Table 8.2). The plan was delivered in 37 fractions, each with a radiation absorbed dose of $2 \mathrm{~Gy}$. The doses were calculated using PB and CC algorithms. 
Table 8.2: Summary of the prostate treatment plan settings for IMRT.

\begin{tabular}{cccccc} 
& \multicolumn{5}{c}{ IMRT plan } \\
\cline { 2 - 6 } & $\begin{array}{c}\text { No of } \\
\text { Segments }\end{array}$ & $\begin{array}{c}\text { Gantry } \\
\text { angle }\end{array}$ & $\begin{array}{c}\text { Collimator } \\
\text { angle }\end{array}$ & SSD & MU \\
\hline BEAM 1 & 5 & 180 & 0 & 86.6 & 76.4 \\
\hline BEAM 2 & 8 & 110 & 335 & 81.4 & 120 \\
\hline BEAM 3 & 6 & 65 & 340 & 83.5 & 101.7 \\
\hline BEAM 4 & 7 & 225 & 25 & 81.1 & 148 \\
\hline BEAM 5 & 4 & 325 & 20 & 88.3 & 123.9 \\
\hline
\end{tabular}

\subsubsection{MC-based treatment plan}

An input file, for each BEAMnrc and DOSXYZnrc, each segment of the IMRT plan (total of 30 segments) was created manually. The simulation or calculation of each segment was performed on the egsphant file, which was generated from the CT and sCBCT DICOM images of the patient (see Section 6.2.6). Then the same steps used in Chapter 7 (see Section 7.2.4) were then used in this chapter.

\subsubsection{Treatment planning evaluation and comparison}

The sCBCT and pCT images were fused using ProSoma software and the structure sets were then transferred to the sCBCT images without any modification except the external contour, where there are some differences. The plans were then copied to sCBCT using the same geometry and MU values and doses were recalculated using $\mathrm{PB}$ and $\mathrm{CC}$ algorithms. For MC calculation, the pCT artefacts (dark shading, as shown in Figure 8.1(e)) were changed to a water material of uniform density using a MATLAB script. The MC dose calculation was then performed on pCT and sCBCT images using the same HU-ED calibration as in OMP (Figure 6.3). The MC dose file (.3ddose) and the DICOM-RT file were then imported into CERR software to compare the resultant dose distribution. 


\subsubsection{DVH and dose profile comparison}

The cumulative DVHs were generated for PTV, rectum and bladder structures and compared between pCT, sCBCT3, sCBCT5 and sCBCT8 plans. The comparison of DVHs was performed using the OMP PB and CC, and MC algorithms. The $\mathrm{D}_{\max }$, $\mathrm{D}_{\text {mean }}$ and $\mathrm{D}_{\text {min }}$ parameters for the above structures were compared. The cross-plane profiles of pCT, sCBCT3 and sCBCT8 plans at the isocentre depth were compared.

\subsubsection{Gamma and conformity index comparison}

To quantitatively appraise the differences between pCT and sCBCT plans, especially for the PTV, rectum and bladder, a $\gamma$ index analysis was performed using the pCT plan as a reference. The criteria were set as $3 \mathrm{~mm}$ DTA and 3\% DD and 5\% low dose threshold. The conformity index (CI), defined as the volume of PTV covered by the reference dose divided by the volume of PTV, was calculated for all SCBCT plans and then compared with the pCT plans using the $\mathrm{PB}, \mathrm{CC}$ and $\mathrm{MC}$ algorithms.

\subsubsection{Dose point comparison}

The dose at the isocentre (at the geometric centre of the prostate PTV (PTVp)) was compared between the $\mathrm{pCT}$ and sCBCT plans and plotted against the operator time required for defining the threshold values for different regions. The greater the variation in scatter the more regions need to be defined. Thus the operator time increases as the number of anatomical materials involved increases. The reason of performing such a comparison was due to the fact that, in the local department, the CBCT images are acquired in the first three days of the radiotherapy treatment and then weekly. If significant changes in patient geometry and/or internal organ position/shape are seen on the CBCT scan, then a dosimetric assessment is required. The original plan is then copied onto CBCT images after creating an external contour and fusing it with the pCT images. The dose is then recalculated turning the inhomogeneity correction off in both scans (see Section 1.3). If the dose difference at the isocentre is within $\pm 2 \%$, the treatment will be continued, whilst if it is more than that the patient will be re-scanned and the original plan is copied onto the new CT. The dose is then recalculated turning the inhomogeneity correction on. The Oncologist will review the dose distribution on the new scan and if acceptable, the 
treatment will be continued, otherwise a new treatment plan will be generated.

\subsection{Result and discussion}

\subsubsection{Dose profile and DVH comparison}

Figure 8.2 shows the cross-plane dose profile of the pCT, sCBCT 8 and sCBCT3 at the depth of the plan isocentre as well as the pCT, sCBCT3 and sCBCT8 number at that depth using (a) PB, (b) $\mathrm{CC}$ and (c) MC algorithms. Only the sCBCT3 and sCBCT8 profiles were plotted to show the clear improvement in the match with the pCT profile when increasing from 3 to 8 values of $\mathrm{HU}$. The subplots represent the absolute differences between $\mathrm{pCT}$ and sCBCT dose profiles to aid comparison.

Whilst the sCBCT3 dose profile clearly showed large difference, it can be seen that the $\mathrm{sCBCT} 8$ profile is in good agreement with the $\mathrm{pCT}$ profile. The largest difference between the pCT and sCBCT8 plans was at the implant/tissue interface where sCBCT8 was $-2.8 \mathrm{~Gy},-3.1 \mathrm{~Gy}$ and $12.1 \mathrm{~Gy}$ when using PB, CC and MC algorithms, respectively. This is due to the fact that the pCT numbers in this region in the $\mathrm{pCT}$ were affected by artefacts due to the presence of the metal. It can be seen that there is a significant drop in the pCT numbers in this region, thus the MC algorithm calculated the dose as if the medium was air, therefore providing lower doses. These artefacts were reassigned as water in the original patient plan using a bulk density correction in the OMP system for dose calculation using the PB and CC algorithms. These artefacts were even more pronounced in the CBCT images, as shown in Figure 8.1 , but they were corrected in the sCBCT8, as well as the sCBCT3 scans. Therefore, the PB and CC algorithms showed relatively small differences $( \pm 3$ Gy) in these regions. It may also be due to the inherent accuracy issues of these algorithms (see Chapter 3). However, the sCBCT3 profile overestimated the dose across the metal by up to 2.20 Gy (20\%), 2.24 Gy (23\%) and 2.98 Gy (36\%) when using PB, CC and MC algorithms, respectively. This is due to the fact that the sCBCT3 approach does not include the HU of metal and thus considers that region as hard bone whilst sCBCT8 considers it as metal. The sCBCT8 numbers overestimated the metal region at that depth compared the pCT numbers. Beside the threshold nature of MLT, the metal artefacts in the pCT scans increased the differences between pCT and sCBCT8 

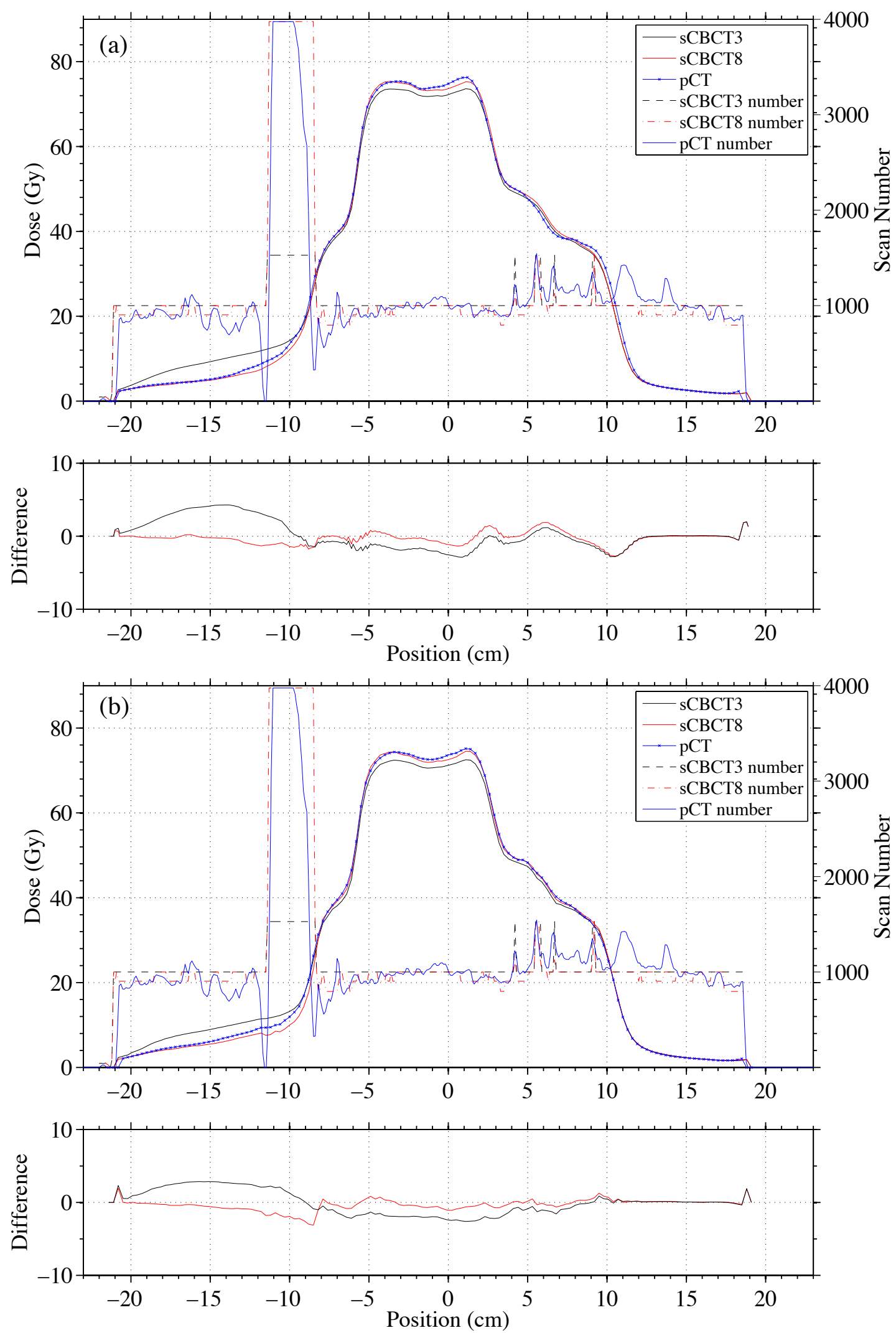

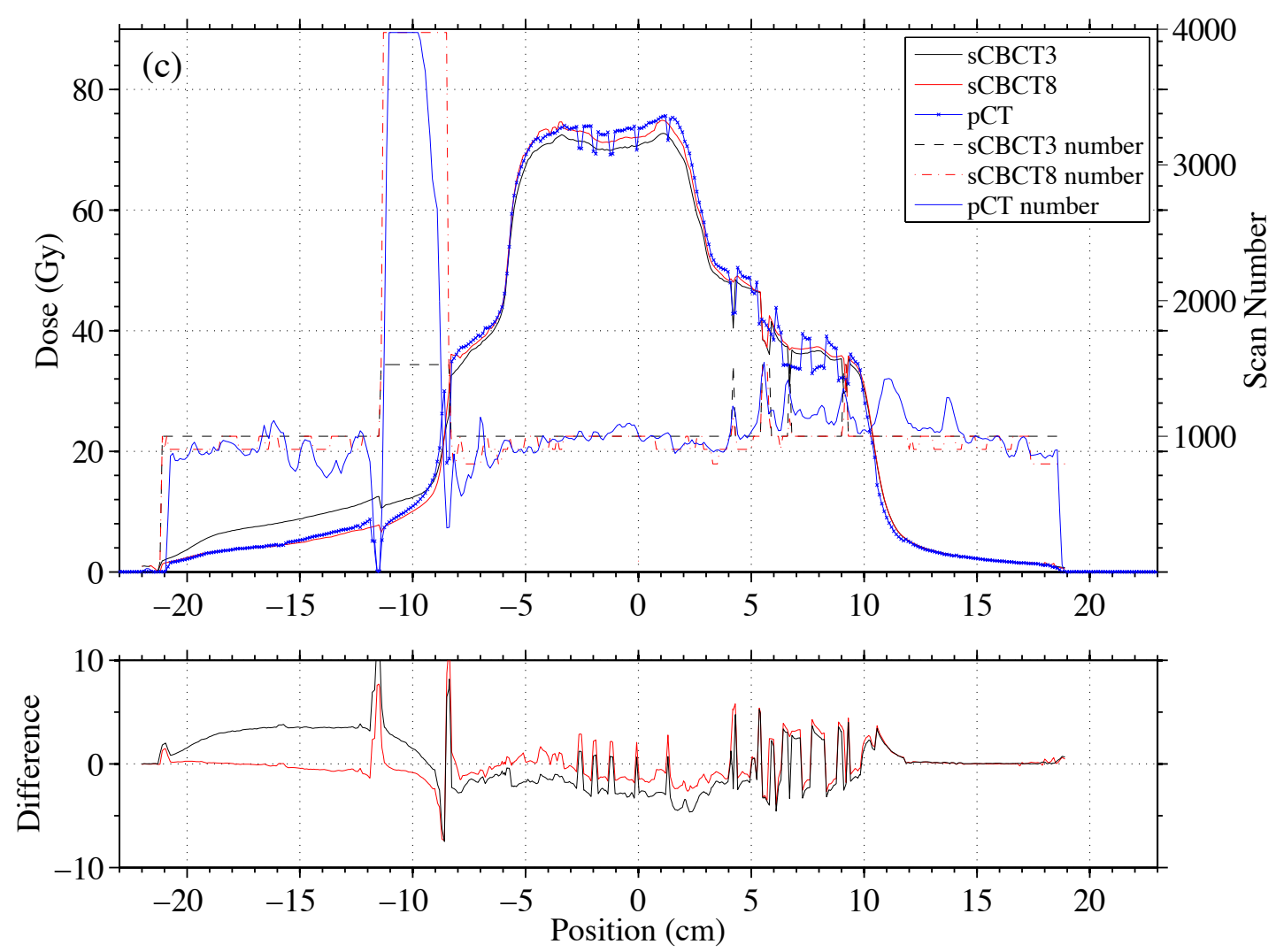

Figure 8.2: Comparison of the dose profile of $p C T, s C B C T 3$ and $s C B C T 8$ plans at the isocentre depth and the absolute difference between sCBCT8 and pCT using (a) PB, (b) CC and (c) MC algorithm.

numbers in that region. However, such differences did not affect the dose in the PTV region as the metal hip was avoided during beam placement.

Figure 8.3 shows the DVH of the prostate IMRT plan. It shows the dose of the sCBCT3, sCBCT5, sCBCT8 and pCT plans to the 95\% volume of the PTV, rectum and bladder using (a) PB, (b) CC and (c) MC algorithms. In terms of PTV coverage, the lowest difference between the pCT and sCBCT plans was achieved using the sCBCT8 plan whilst the largest difference was obtained using the sCBCT3 plan, which provided less anatomical materials compared with sCBCT8. The results showed that the differences between pCT and SCBCT increased as the number of bins decreased. Table 8.3 shows the dose and coverage differences between sCBCT plans and pCT plan for the PTV, rectum and bladder using PB, CC and algorithms. In general, the higher the number of HU bins the better the agreement with the pCT. There are some differences and outlines but the general trend holds. 

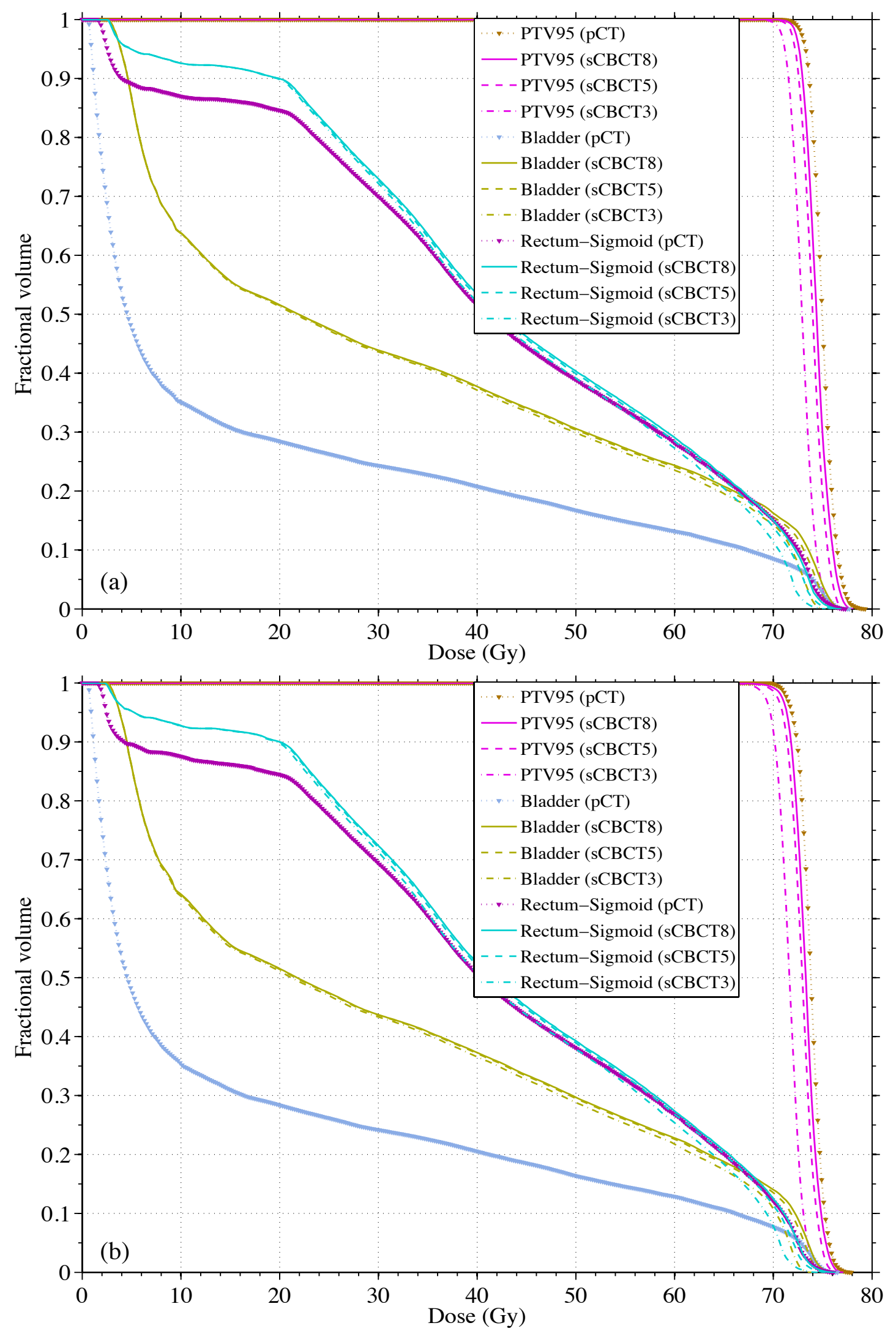


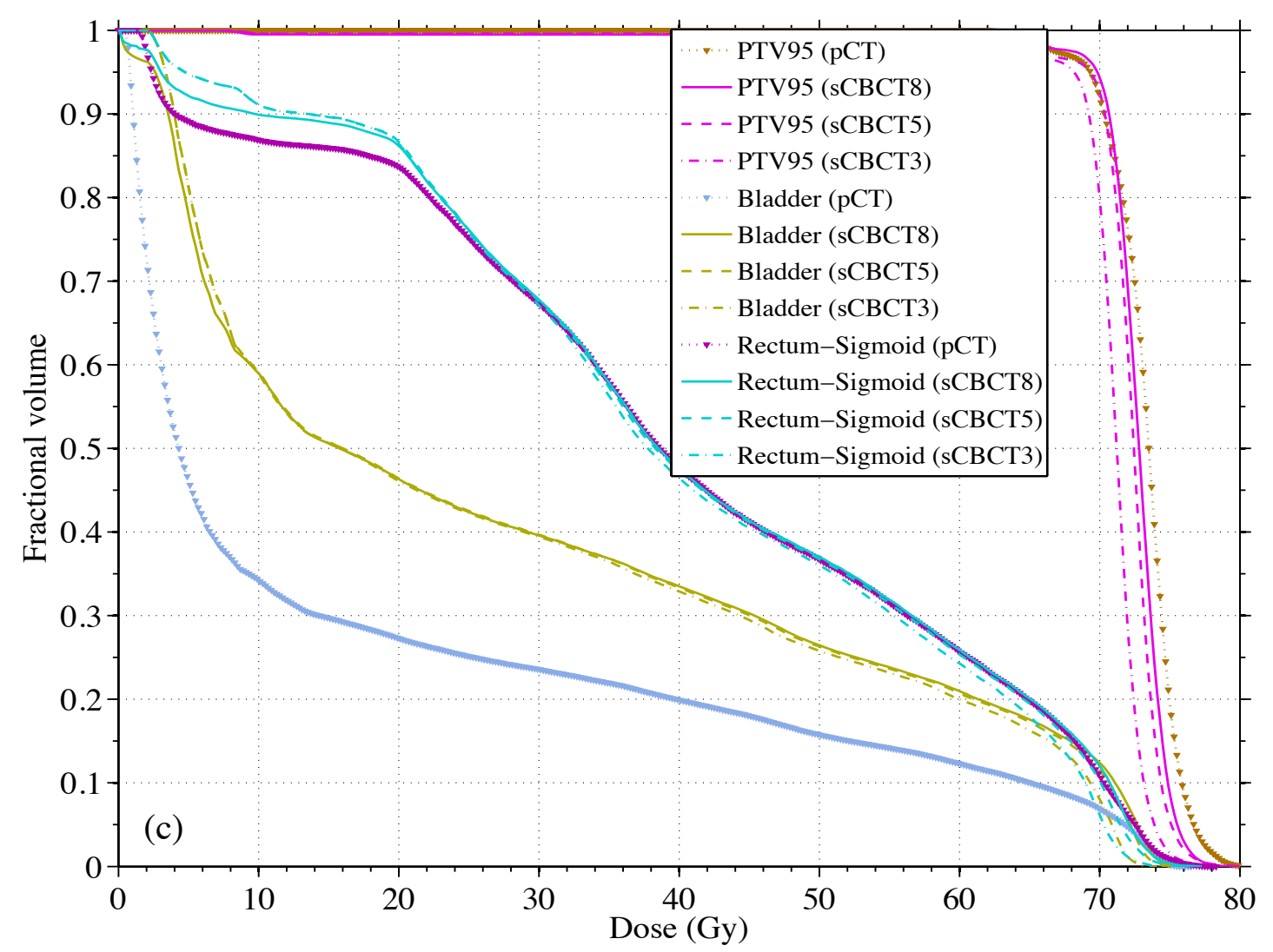

Figure 8.3: DVHs comparison pCT (:v), sCBCT8 (solid line), sCBCT5 (--) and sCBCT3 (-.) IMRT plans for PTV95, rectum and bladder using (a) $P B,(b) C C$ and (c) MC algorithm.

It is worth mentioning that there are some differences in the bladder and rectal volume between the pCT and CBCT scans. The bladder volume, for example, is significantly reduced in the CBCT scan ( $>25 \%$ reduction). Therefore, the differences are not directly comparable indicting that deformable image registration (DIR) was needed rather than rigid image registration to correct for organ deformation between $\mathrm{pCT}$ and CBCT. Currently, only rigid image registration is available for clinical use. Deforming the pCT to match the CBCT anatomy so that the original HU are copied onto the CBCT could be used as a benchmark for the study. Yang et al (2007) used deformable electron density mapping on CBCT images and reported that the dose difference between pCT and CBCT was within $2 \%$ in three prostate patients. More recently, Onozato et al (2014) used the MLT algorithm as well as DIR on CBCT images of ten prostate patients and achieved better accuracy $(<1 \%)$. For some patients, the accuracy was not improved mainly due to the large artefacts from gold fiducial markers and bowel gas in CBCT images, which could have been corrected if the threshold values were changeable geometrically. None of these studies included a patient with a hip prosthesis. 
Table 8.3: Dose and coverage differences between sCBCT plans and $\mathrm{pCT}$ plan in \% for the $P T V$, rectum and bladder using $P B, C C$ and $M C$ algorithms.

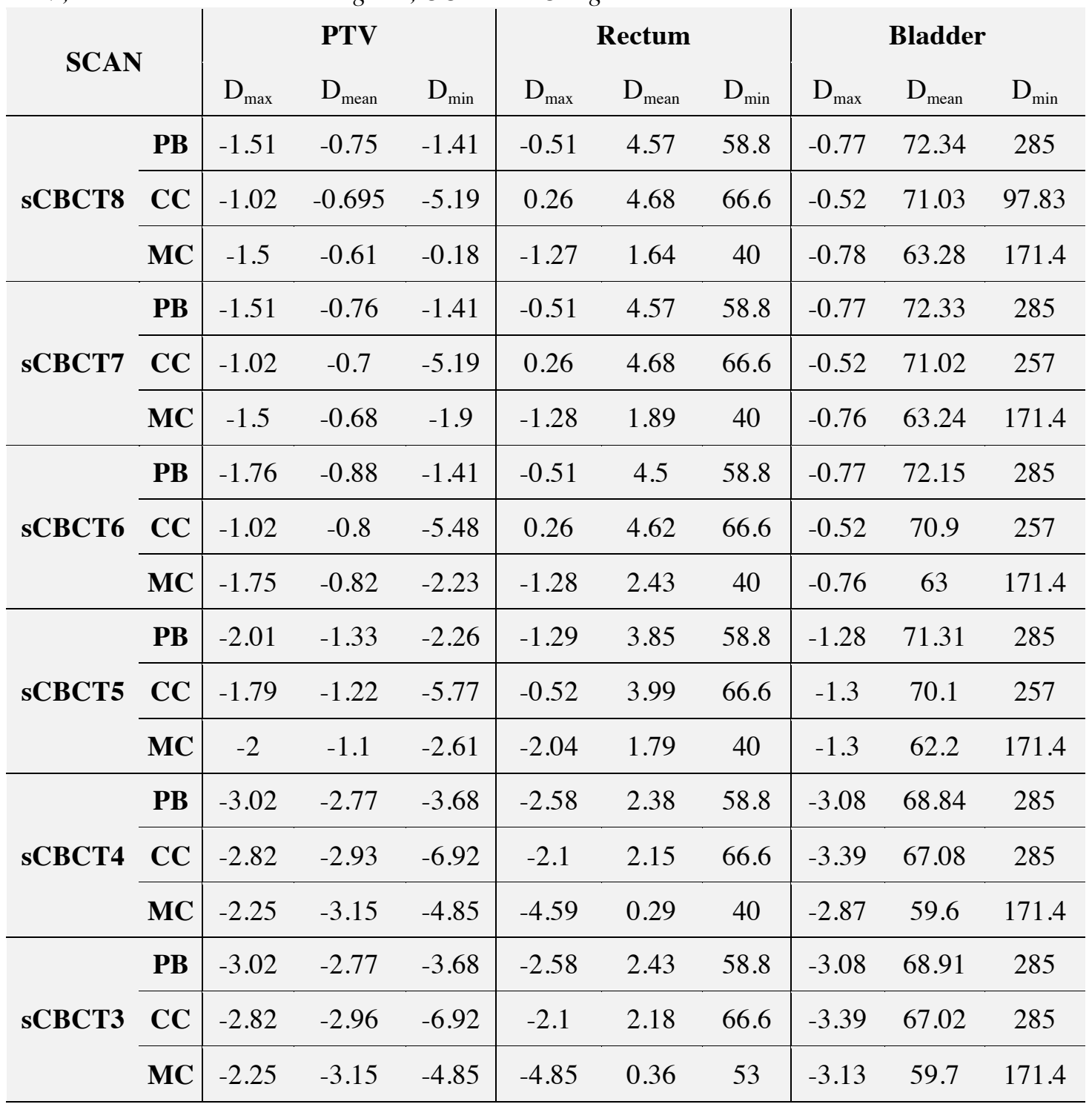

\subsubsection{Gamma and conformity index comparison}

Figure 8.4(a) shows the $\gamma$ index evaluation for the calculation points falling inside the PTV, rectum and bladder for different bins, showing the fraction of points resulting with $\gamma<1$. In general, as the number of bins decreased the number of calculation points which passed $(\gamma<1)$ decreased slightly at first and then significantly when moving from 5 to 4 bins for all algorithms. The number of points that passed remained almost unchanged when going from 4 to 3 bins except for the rectum region where it increased from $99.3 \%$ to $99.8 \%$ when using PB and from $97.9 \%$ to $98.2 \%$ when using CC algorithm. For the PTV and bladder region, all the calculation points passed the gamma test for 5 up to 8 bins when using the PB algorithm, whilst using 

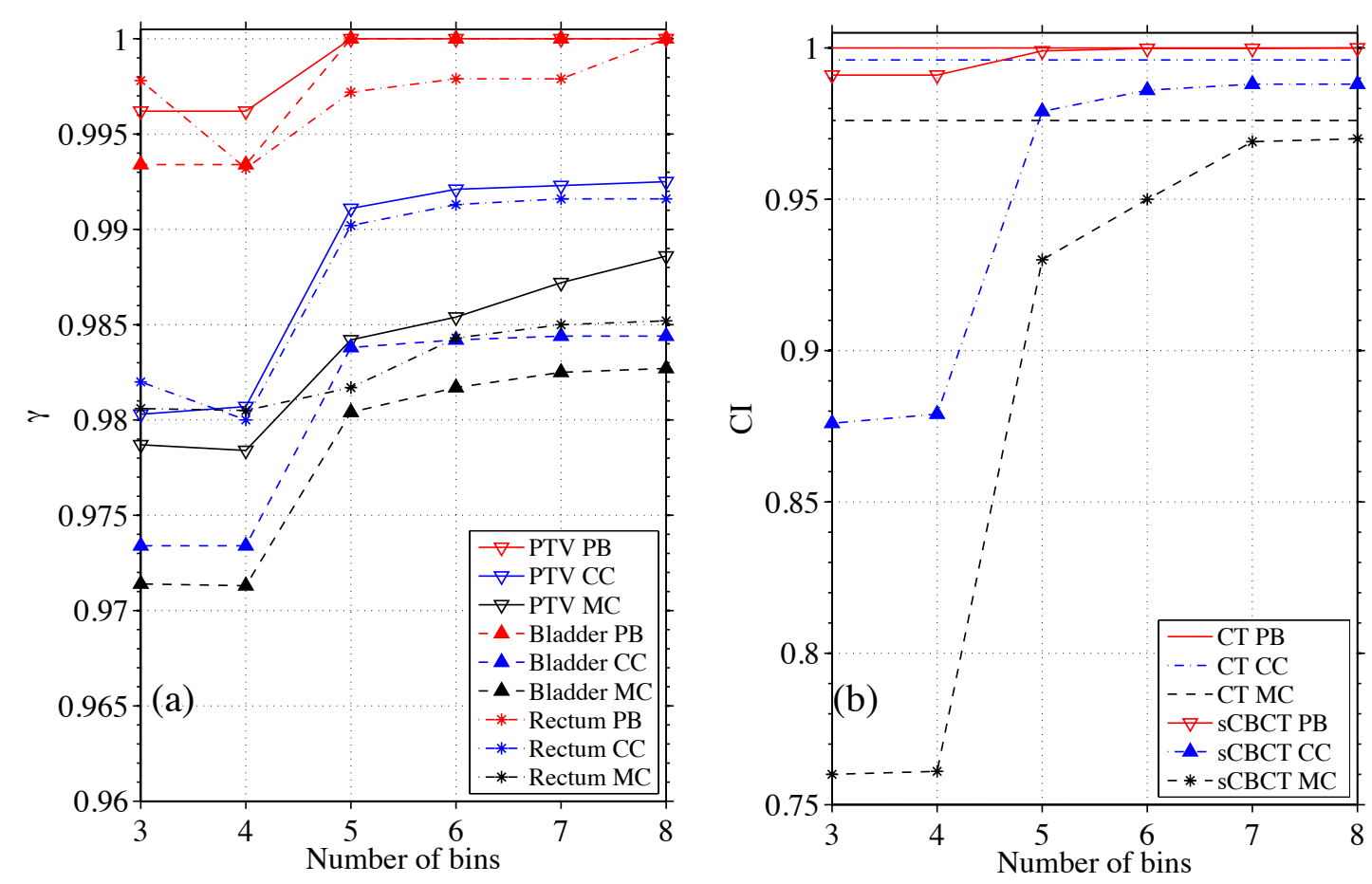

Figure 8.4: (a) Summary of the $\gamma$ index with fixed DTA $=3 \mathrm{~mm}$ and $D D=3 \%$ for the calculation points falling inside the PTV, rectum and bladder, showing the fraction of points resulting with $\gamma<1$. (b) CI comparison between $p C T$ and $s C B C T$ plans using $P B, C C$ and $M C$ algorithms against number of bins.

the CC algorithm, more than $99.1 \%$ showed $\gamma<1$ for the PTV, and more than $98.3 \%$ for the bladder, when using the same bins. When using MC, the number of calculation points that showed $\gamma<1$ decreased almost linearly as the number of bins decreased. As mentioned before, the PB algorithm in OMP calculates dose to water whilst, the $\mathrm{CC}$ algorithm calculates dose to medium as does the MC algorithm (Knöös et al., 2006). Since MC and CC algorithms calculate dose to medium, the HU of the medium must be provided precisely. Therefore, the PB algorithm is less sensitive than $\mathrm{CC}$ and $\mathrm{MC}$ for calculating the dose using different bins as shown in Figure 8.4. Thus MC and CC algorithms minimised uncertainty related to the dose calculation as well as identifying those introduced by different bins.

Figure 8.4(b) shows the CI values of the sCBCT plans and the difference compared with pCT using PB, CC and MC algorithms. The three horizontal lines indicate the $\mathrm{CI}$ value of the $\mathrm{pCT}$ plan using $\mathrm{PB}, \mathrm{CC}$ and $\mathrm{MC}$ algorithms. The $\mathrm{CI}$ value changed very little when going from the sCBCT8 to the sCBCT7 plan using all algorithms. The most significant change in the CI value was found when moving from the sCBCT5 plan to the sCBCT4 plan, going from 0.98 to 0.87 when using the $\mathrm{CC}$ algorithm and 


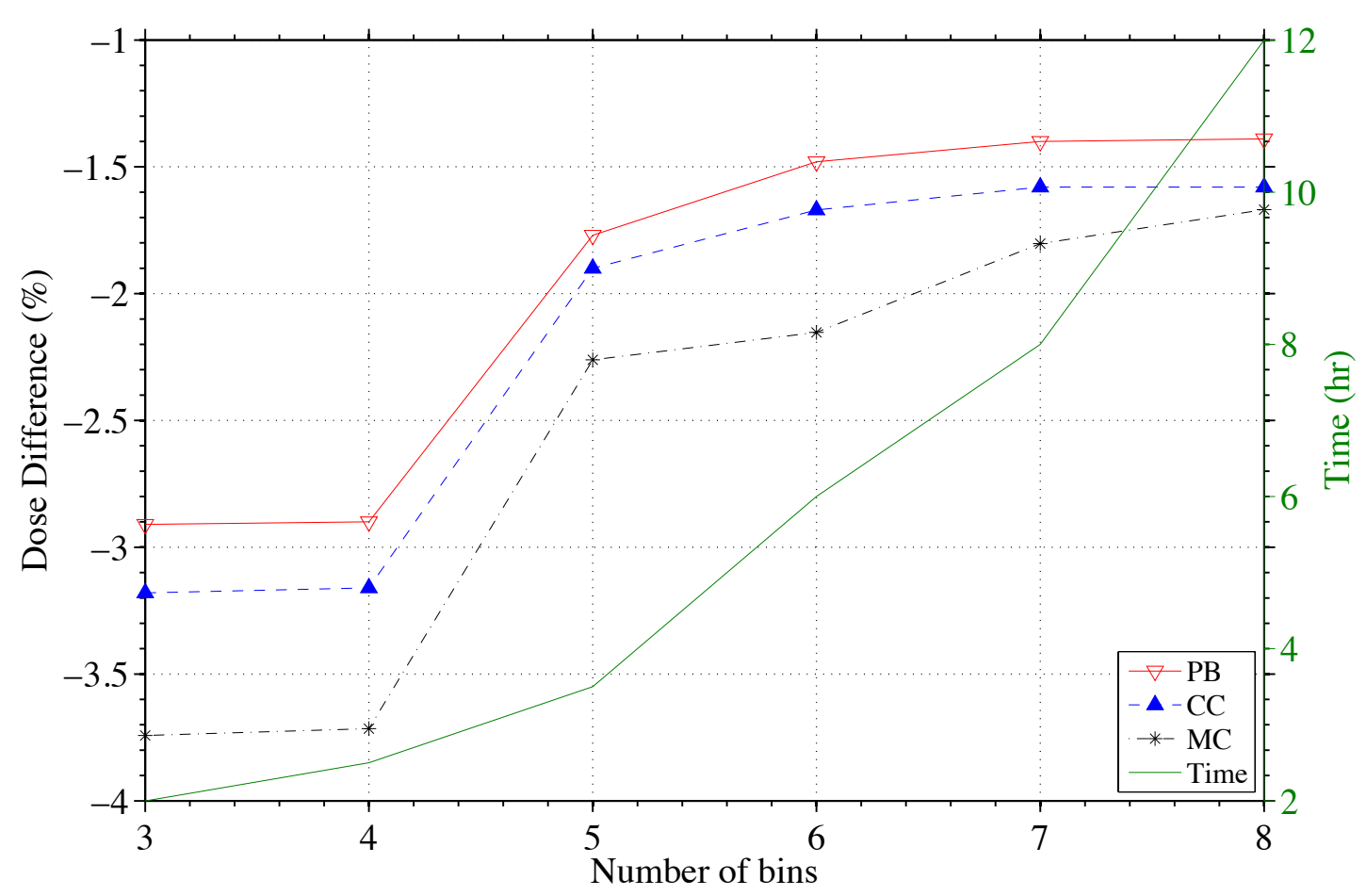

Figure 8.5: Dose comparison between $p C T$ and sCBCT plans at the isocentre against operator time using $P B, C C$ and $M C$ algorithms.

from 0.93 to 0.76 when using the MC algorithm. The results showed that going for fewer than 5 bins for such a case would cause a difference of at least $13 \%$ in the CI values compared with $\mathrm{pCT}$ when using $\mathrm{CC}$ and $\mathrm{MC}$ algorithms. According to the RTOG guidelines, a CI value between 0.9 and 1 indicates that a target volume is not adequately covered by the prescribed isodose with a minor violation, whereas for CI values of less than 0.9 the treatment plan are rated as having major violations but may nevertheless be considered to be acceptable (Feuvret et al., 2006, Petkovska et al., 2010).

\subsubsection{Dose point comparison}

Figure 8.5 shows the dose difference between pCT and sCBCT plans at the isocentre using all algorithms plotted against the operator time needed to segment each sCBCT bin. The sCBCT5 to sCBCT8 plans showed differences of less than $-2 \%$ compared with the pCT plan when using the $\mathrm{PB}$ and $\mathrm{CC}$ algorithms, which is considered to be clinically acceptable. For the MC algorithm, only the sCBCT7 and sCBCT8 plans showed similar differences. It can be clearly seen that as the number of bins increased the operator time increased as shown in Figure 8.5. From sCBCT7 (8 hours) to 
sCBCT8 (12 hours), it required 50\% more operator time to improve the accuracy by $0.01 \%$ when PB and CC algorithms were used for dose calculation and $0.05 \%$ when using MC algorithm in this case (other cases may be different). For sCBCT5 (3.5 hours) plan, compared with $\mathrm{SCBCT} 7$, it required about 55\% less operator time to improve the accuracy by $1.13 \%, 1.26 \%$ and $1.45 \%$ using PB, CC and MC algorithms respectively. Therefore, the five bins is the optimal level which balances the accuracy of the calculation and the time required. This time would be greatly reduced with automation but there is likely to be a certain amount of operator intervention required and the relative amount of operator time is likely to be dependent on the number of bins chosen (see Chapter 10). Furthermore, a patient with double metal hip prostheses would complicate the segmentation process and the operator time is expected to be longer, as shown in the next chapter.

\subsection{Conclusion}

The segmentation of CBCT images using the MLT method in this study can be used for dose calculation. In this chapter, the segmentation of CBCT of a prostate patient with hip prosthesis, where the irradiated volume has a large range of inhomogeneities (including metal prosthetic implant), was investigated. It was necessary to extend the MLT algorithm to categorise pixel values into segments on a region-by-region basis, with the region size changing depending on the anatomical features as there is a variation on a slice by slice basis. In addition, a larger number of materials (up to 8) than typically used in previous works was explored. The results showed that five values of HU (air, adipose, water, hard bone and metal implant) gave the best balance between dose accuracy $(-1.9 \%)$ and operator time (3.5 hours). Thus this method is feasible for ART, as an alternative to obtaining a new planning CT and re-outlining the structures, which can take up to a day in a busy radiotherapy department, and more importantly avoiding the delivery of additional dose. This chapter and parts of Chapter 6 (as a phantom case) have been published (Almatani et al., 2016a). In the next chapter, a more complicated case, as a prostate patient with double hip prostheses, will be investigated. 
Chapter 9 CBCT-based dose calculation of a prostate cancer patient with double hip prostheses 


\subsection{Introduction}

In the previous chapter, the segmentation of CBCT images of a prostate patient with single hip prosthesis was investigated. It was necessary to extend the MLT algorithm to categorise pixel values into segments on a region-by-region basis, with the region size changing depending on the anatomical features. In general, the greater the variation in the scatter the greater the number of regions that need to be considered and the size of the region decreases as it gets closer to inhomogeneities.

The results showed that the optimal amount of operator time, which balanced the dose accuracy and calculation time, was found when only five values of $\mathrm{HU}$, including air, adipose, water, bone and metal implant HU values, were used. However, the occurrence of more inhomogeneities in the patient anatomy, e.g. another hip prosthesis, would complicate the segmentation process. The amount of scatter increases with the presence of the two metallic hips, where the highly attenuating material results in even more image artefacts. If there are two prostheses within the scanned patient, the metal artefacts caused by the beam hardening effect appear as dark streaks between the metals with surrounding bright streaks as shown in Figure 9.1. In addition, the metal implant may also totally attenuate or absorb all the incoming photons along certain beam paths. Thus the detector signal, in such a situation, is zero leading to zero-data artefacts (see Section 2.5.4). In this chapter, the segmentation of a prostate cancer patient with bilateral metallic prosthetic hip replacements was investigated. Based on the results of the previous chapter, only five bins were used in this chapter. The main aim of this chapter was to ensure that the MLT method works for patients with two hip implants and to find the optimal operator time required to achieve a dose accuracy that is clinically acceptable.

\subsection{Methodology}

\subsubsection{CT and CBCT image acquisition}

A prostate cancer patient with bilateral metallic prosthetic hip replacements was imaged using the same CT scanner used in the previous chapters with the same setting except that the matrix size of the reconstructed images was $512 \times 512$ and voxel sizes were $1.06 \times 1.06 \times 3 \mathrm{~mm}^{3}$. The scan length in the superior-inferior direction was 33.6 


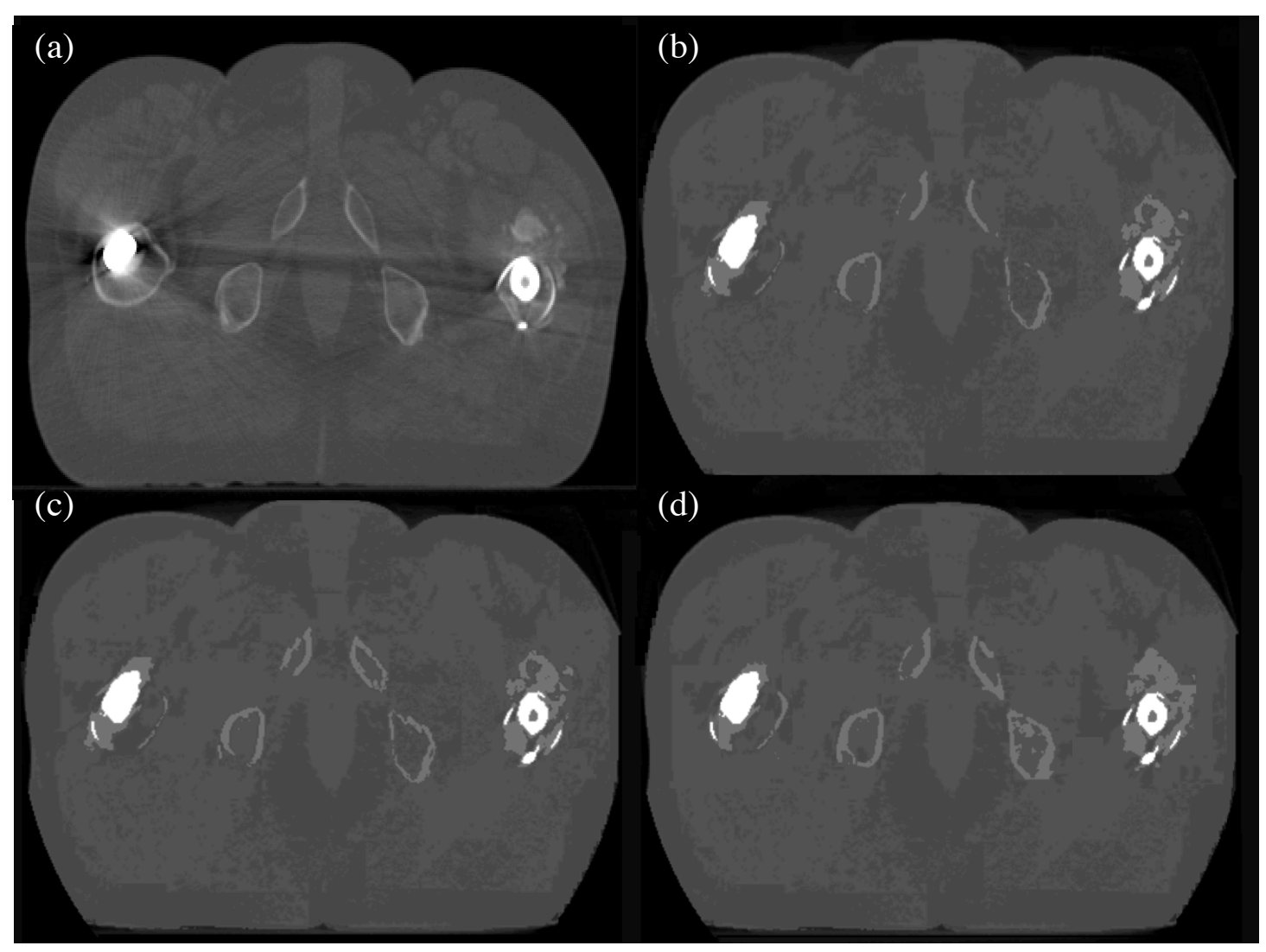

Figure 9.1: A prostate cancer patient with bilateral hip replacement using (a) $p C T$ and the resultant images after segmentation (b) $1^{s t} s C B C T$, (c) $2^{\text {nd }} s C B C T$ and $(d) 3^{\text {rd }} s C B C T$.

$\mathrm{cm}$. A CBCT scan was acquired with a MFOV, collimator M15 and the F1 filter using the following parameters: $120 \mathrm{kVp}, 25 \mathrm{~mA}, 40 \mathrm{~ms}$ per projection and 660 projections. The matrix size of the reconstructed images was $410 \times 410$, the voxel size was $1 \times 1 \times$ $3 \mathrm{~mm}^{3}$ and the scan length in the superior-inferior direction along the phantom (G-T direction) was $16.8 \mathrm{~cm}$.

\subsubsection{Modification of CBCT image}

The CBCT images were segmented on a region-by-region basis, with the region size changing depending on the anatomical features. For the single hip prosthesis, segmenting the CBCT images into five bins achieved an acceptable dose accuracy ($1.9 \%$ ). The operator time needed to achieve that accuracy was 3.5 hours. In this chapter, the operator time of segmenting CBCT images into five material bins was 8 hours as the variation in the scatter was greater than in the single hip case, due to the presence of the metallic hips in both sides. Thus the number of regions that need to be considered was greater. Due to the increased amount of scatter, more time was 
required in the segmentation to achieve an accurate representation of the bony structures. Furthermore, two additional $\left(2^{\text {nd }}\right.$ sCBCT and $3^{\text {rd }}$ sCBCT) segmentations of the same CBCT images were performed (Figure 9.1). The operator time of segmenting $2^{\text {nd }} \mathrm{sCBCT}$ and $3^{\text {rd }} \mathrm{sCBCT}$ was 12 and 14 hours, respectively (more time to delineate bone regions). The main difference between the three sCBCT images was mostly the volume of the bony structures considered in the segmentation process, as shown in Figure 9.1.

\subsubsection{OMP-based treatment plan}

Using the inverse planning option in the OMP software, a segmented IMRT plan with five 6-MV photon fields, at gantry angles of $180^{\circ}, 145^{\circ}, 35^{\circ}, 235^{\circ}$ and $300^{\circ}$ was performed on the pCT image set (Table 9.1). The plan was delivered in 35 fractions, each with an absorbed radiation dose of $2 \mathrm{~Gy}$. The fusion of the sCBCT and pCT images was accomplished with manual rigid registration using ProSoma software. The structure sets were then transferred to the sCBCT images without any modification except the external contour. Then the same steps described in Chapter 7 and 8 were used in this chapter.

Table 9.1 Summary of the treatment plan settings for IMRT.

\begin{tabular}{cccccc} 
& \multicolumn{5}{c}{ IMRT plan } \\
\cline { 2 - 6 } & $\begin{array}{c}\text { No of } \\
\text { Segments }\end{array}$ & $\begin{array}{c}\text { Gantry } \\
\text { angle }\end{array}$ & $\begin{array}{c}\text { Collimator } \\
\text { angle }\end{array}$ & SSD & MU \\
\hline BEAM 1 & 8 & 180 & 0 & 85.1 & 84.4 \\
\hline BEAM 2 & 3 & 145 & 340 & 81.8 & 63.1 \\
\hline BEAM 3 & 4 & 35 & 345 & 88.2 & 77.9 \\
\hline BEAM 4 & 4 & 235 & 18 & 78.4 & 85.4 \\
\hline BEAM 5 & 4 & 300 & 20 & 82.9 & 100.4 \\
\hline
\end{tabular}

\subsubsection{MC-based treatment plan}

Input files, for each BEAMnrc and DOSXYZnrc, were created manually for each segment of the IMRT plan (total of 23 segments). The simulation or calculation of each segment was performed on the egsphant file, which was generated from the $\mathrm{pCT}$ 
and sCBCT DICOM images of the patient (see Section 6.2.6). Then the same steps described in Chapter 7 and 8 were used in this chapter.

\subsubsection{Treatment planning evaluation and comparison}

The sCBCT and pCT images were fused using ProSoma software and the structure sets were then transferred to the sCBCT images without any modification except for the external contour. The plans were then copied to SCBCT using the same geometry and MU values and the doses were recalculated using PB and CC algorithms. For the MC calculation, the pCT artefacts (Figure 9.1(a)) were changed to a water material of uniform density using a MATLAB script. Then the same precedure as in the previous Chapter 8 was used.

\subsubsection{DHV comparison}

The cumulative DVHs were generated for PTV (the prostate and seminal vesicles), rectum and bladder structures and compared between $\mathrm{pCT}, 1^{\text {st }} \mathrm{sCBCT}, 2^{\text {nd }} \mathrm{sCBCT}$ and $3^{\text {rd }}$ sCBCT plans. The comparison of DVHs was performed using OMP, PB and CC, and $\mathrm{MC}$ algorithms. The $\mathrm{D}_{\max }, \mathrm{D}_{\text {mean }}$ and $\mathrm{D}_{\min }$ parameters for the above structures were compared.

\subsubsection{Hip and bone volume comparison}

As the main difference between the three sCBCT images was mostly the volume of the bony structures, the bone volumes in the left and right side in the $\mathrm{pCT}$ images were compared with those in the $1^{\text {st }} \mathrm{sCBCT}, 2^{\text {nd }} \mathrm{sCBCT}$ and $3^{\text {rd }} \mathrm{sCBCT}$ images. Furthermore, the cumulative intensity or image volume histogram (IVH) of the left and right hip structures was compared using CERR. For the IVH comparison, only $1^{\text {st }}$ sCBCT data was used since the volume of the left and right hip in the three sCBCT image sets was the same.

\subsubsection{Gamma and conformity index comparison}

To quantitatively appraise the differences between pCT and sCBCT plans, especially for the PTV, rectum and bladder, a gamma index analysis was performed using the 
pCT plan as a reference. The same criteria used in the previous chapters were used. In addition, the CI was calculated for all sCBCT plans and then compared with the pCT plans using PB, CC and MC algorithms.

\subsubsection{Dose point comparison}

The dose at the isocentre (at the geometric centre of the prostate PTV (PTVp)) was compared between the $\mathrm{pCT}$ and sCBCT plans and plotted against the operator time required for defining the threshold values for different regions. As the main difference between the three sCBCT images was mostly the volume of the bony structures, the greater the operator time invested (to delineate bone regions) the more accurate the representation of these bony structures.

\subsection{Result and discussion}

\subsubsection{DVH comparison}

Figure 9.2 shows the DVH of a prostate IMRT plan with a prescription dose of $70 \mathrm{~Gy}$. It shows the dose of $1^{\text {st }} \mathrm{sCBCT}, 2^{\text {nd }} \mathrm{sCBCT}, 3^{\text {rd }} \mathrm{sCBCT}$ and $\mathrm{pCT}$ plans to the PTV, rectum and bladder using (a) PB, (b) CC and (c) MC algorithms. In terms of PTV coverage, the smallest difference between the $\mathrm{pCT}$ and $\mathrm{sCBCT}$ plans was achieved by the $1^{\text {st }}$ sCBCT plan whilst the largest difference was obtained by the $3^{\text {rd }} \mathrm{sCBCT}$. In general, going from the $1^{\text {st }} \mathrm{sCBCT}$ to the $3^{\text {rd }} \mathrm{sCBCT}$ plans, the differences with the pCT plan increased, as shown in Table 9.2. For example, compared with the pCT plan, the PTV mean dose was underestimated by $-0.5 \%,-0.8 \%$ and $-1.6 \%$ in the $1^{\text {st }}$ $\mathrm{sCBCT}, 2^{\text {nd }} \mathrm{sCBCT}$ and $3^{\text {rd }} \mathrm{sCBCT}$ plans, respectively, when using the MC algorithm. The $1^{\text {st }} \mathrm{sCBCT}, 2^{\text {nd }} \mathrm{sCBCT}$ and $3^{\text {rd }} \mathrm{sCBCT}$ plans underestimated the rectum mean dose by $-2.3 \%,-2.4 \%$ and $-3.1 \%$, respectively. Some differences in the bladder and rectal volume between the $\mathrm{pCT}$ and $\mathrm{CBCT}$ scans were observed due to physical changes in the patient anatomy. The bladder volume, for example, was significantly reduced in the CBCT scan (> $15 \%$ reduction). Such a deformation resulted in larger differences in the bladder mean dose between the pCT and all sCBCT plans (Table 9.2). Therefore, the differences are not directly comparable (see Section 8.3.1). 

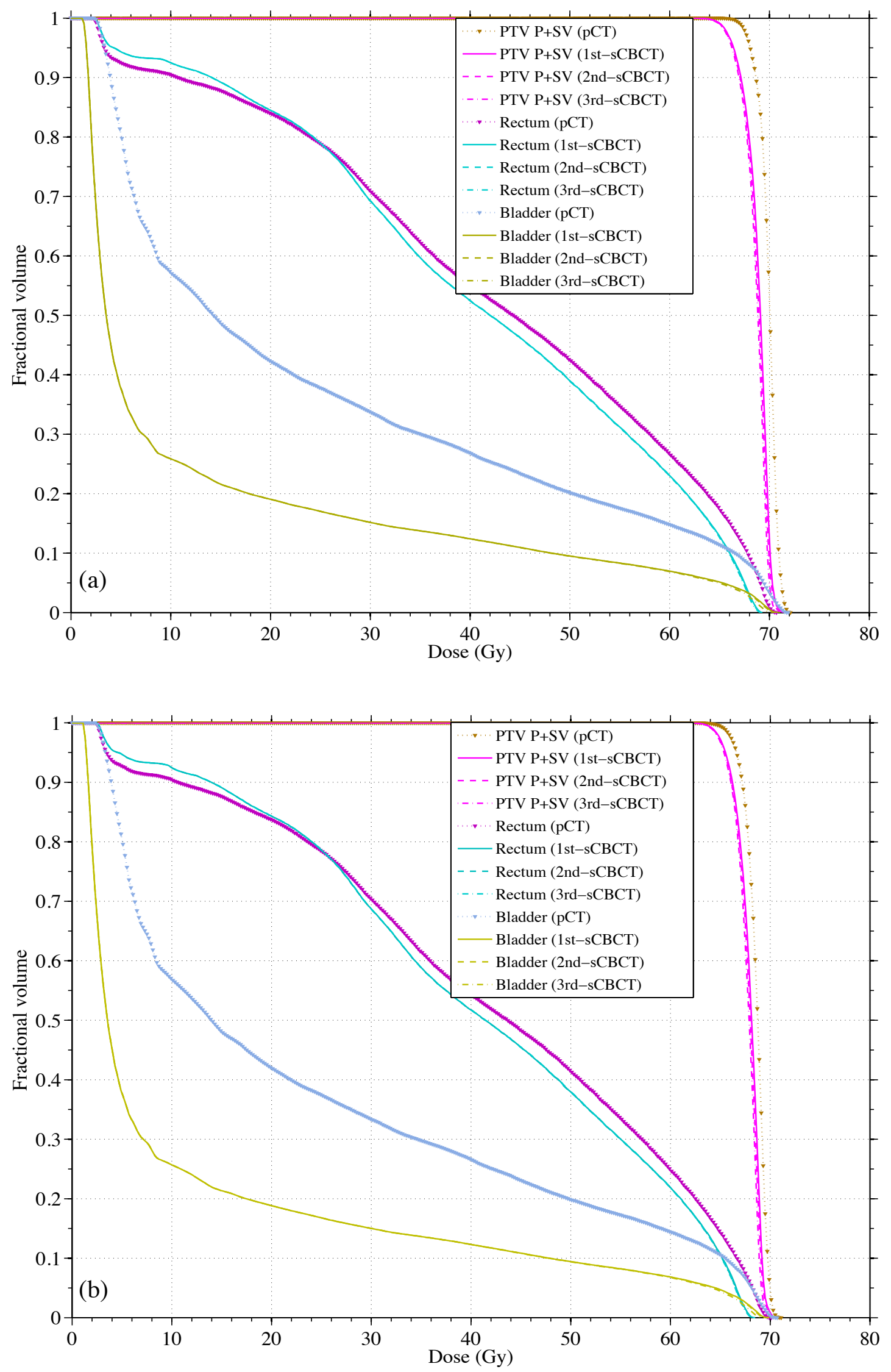


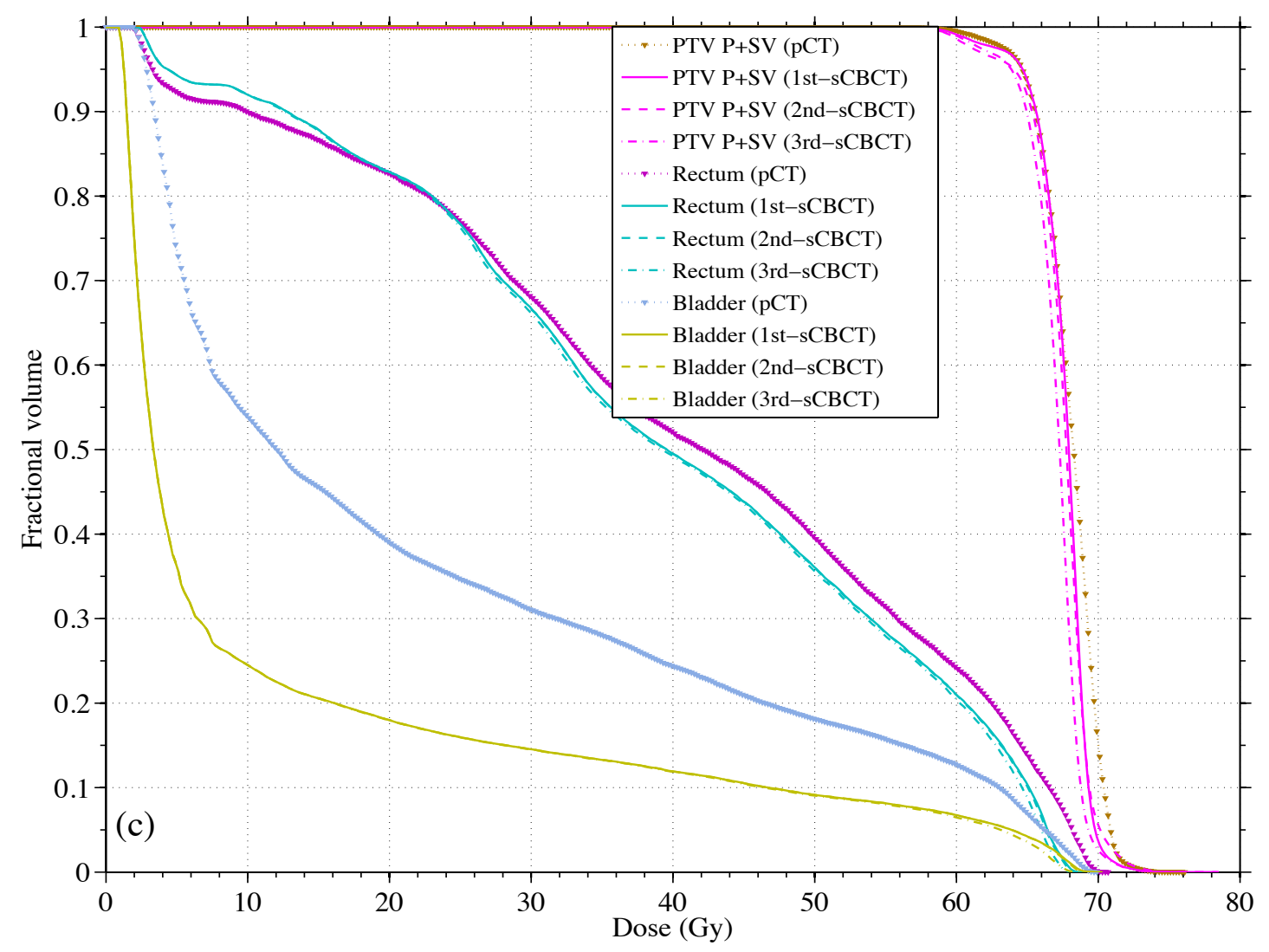

Figure 9.2: DVHs comparison $p C T, 1^{\text {st }} s C B C T, 2^{\text {nd }} s C B C T$ and $3^{\text {rd }} s C B C T$ IMRT plans for $P T V 95$, rectum and bladder using (a) $P B,(b) C C$ and (c) MC algorithms.

Table 9.2: Dose and coverage differences between the sCBCT plans and the pCT plan, in \%, for the PTV, rectum and bladder using $P B, C C$ and $M C$ algorithms.

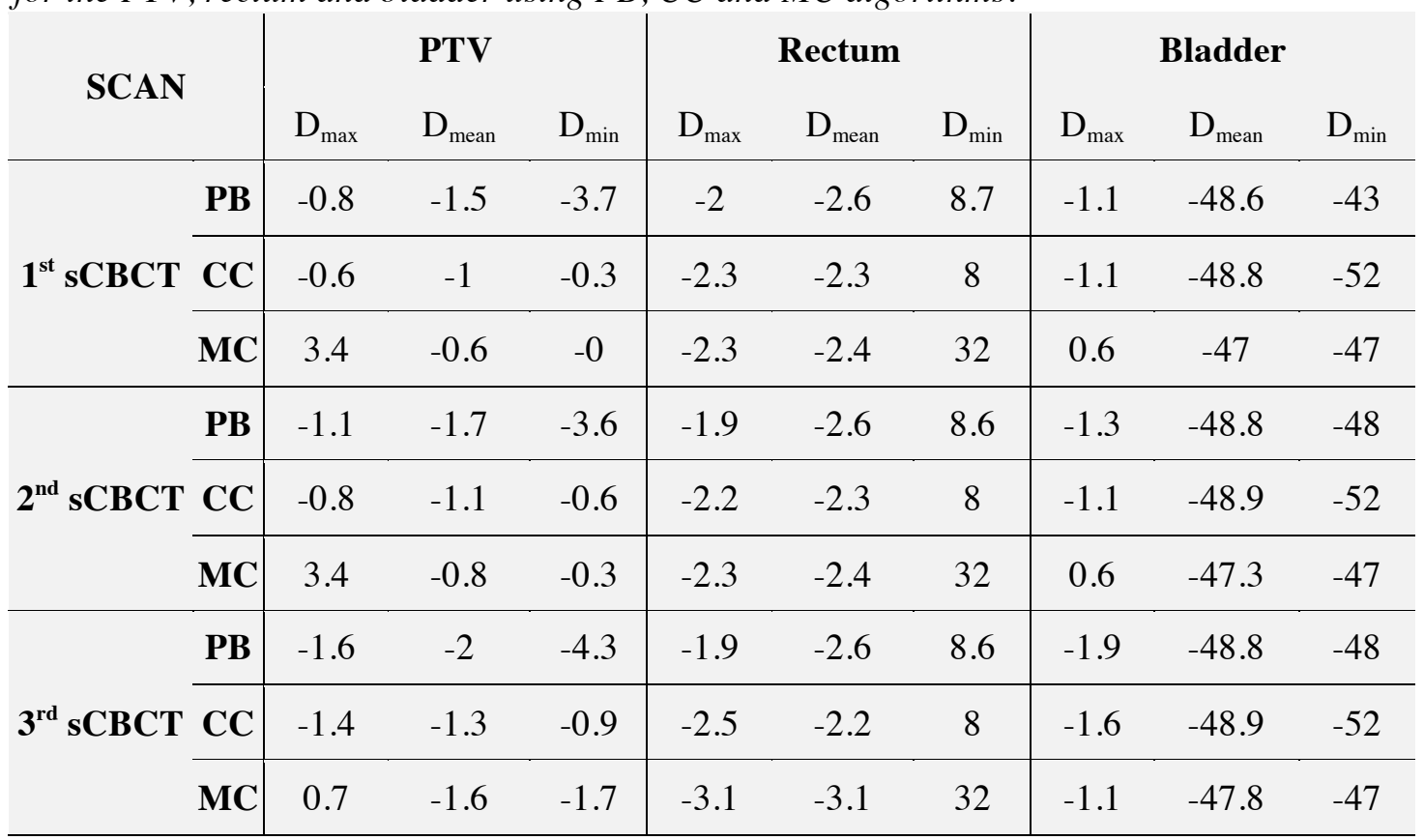




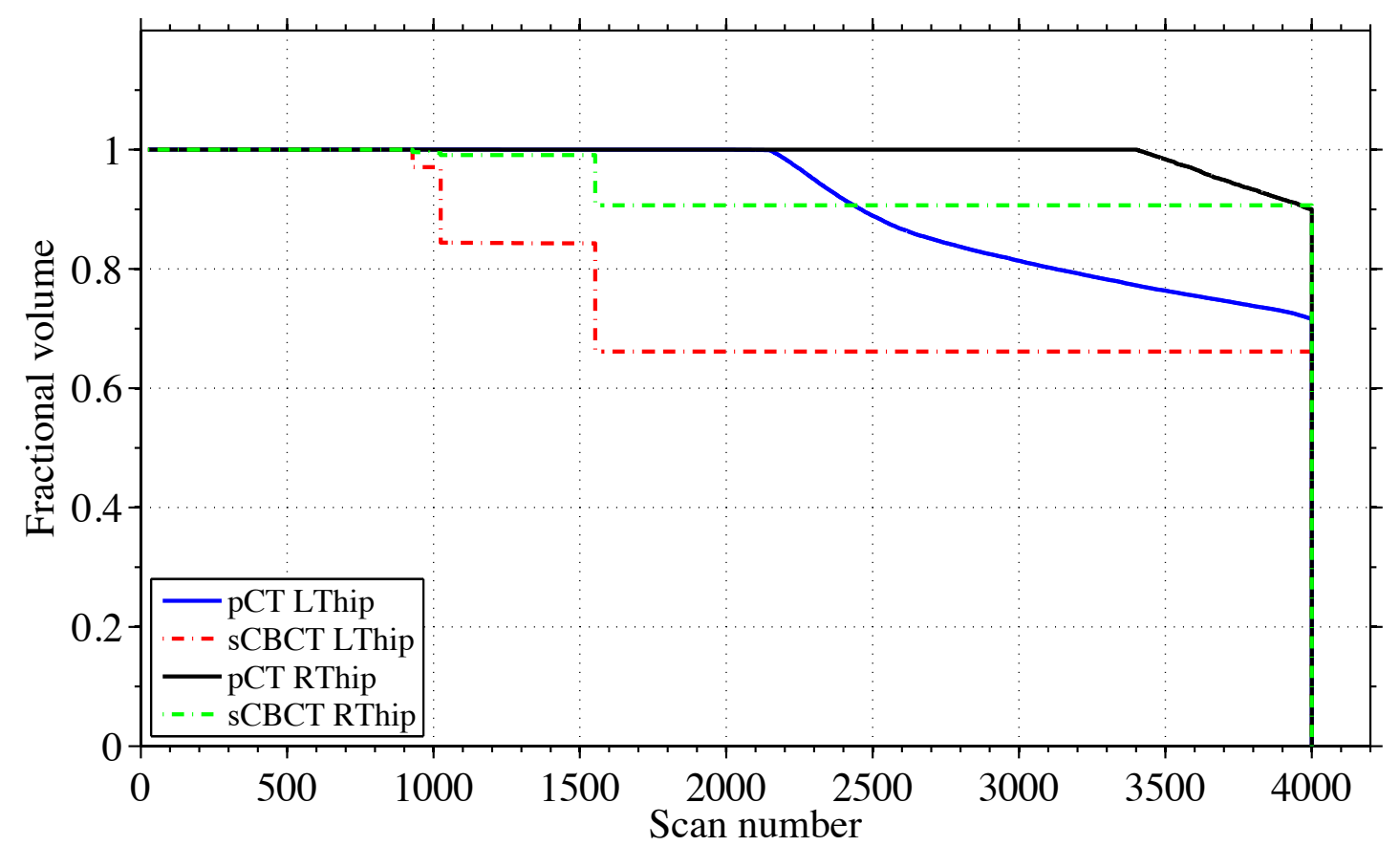

Figure 9.3: Image volume histogram (IVHs) comparison $p C T$ and $1^{\text {st }} s C B C T$ for left $(L T)$ and right (RT) hip.

\subsubsection{Hip and bone volume comparison}

Figure 9.3 shows the cumulative IVH of the right (RT)/left (LT) hip structures of the pCT and sCBCT scans. Since the two hip volumes (metallic hip material bin) were the same in the three sCBCT scans, only data from the $1^{\text {st }}$ SCBCT was used in the IVH comparison. The result showed that the sCBCT scan considered 2.9\%, $12.6 \%$ and $18.3 \%$ of the LT hip structure as adipose, water and bone, respectively. In the pCT scan, about $30 \%$ of the LT hip volume showed pCT numbers that were higher than the bone material bin (1552) and increased gradually from 2170 to 4000 . This was due to the partial volumes effect, or the metal artefacts, within the LT hip structure. In the sCBCT scan, such an effect was not presented properly and it was therefore partially considered as low density materials such as adipose and water. However, the sCBCT scan considered $66.2 \%$ of the LT hip volume as metal implant (with a sCBCT number 4000) which is comparable with the pCT scan (where 70\% was considered to be as metal implant). In addition, the sCBCT scan considered $0.4 \%$, $0.5 \%, 8.5 \%$ and $90.6 \%$ of the volume of the RT hip structure as adipose, water, bone and metal implant, respectively. Similarly, the pCT scan considered $90 \%$ of the volume of the RT hip structure as metal implant but the rest of the volume (10\%) showed pCT numbers that increased gradually from 3402 to 4000. 


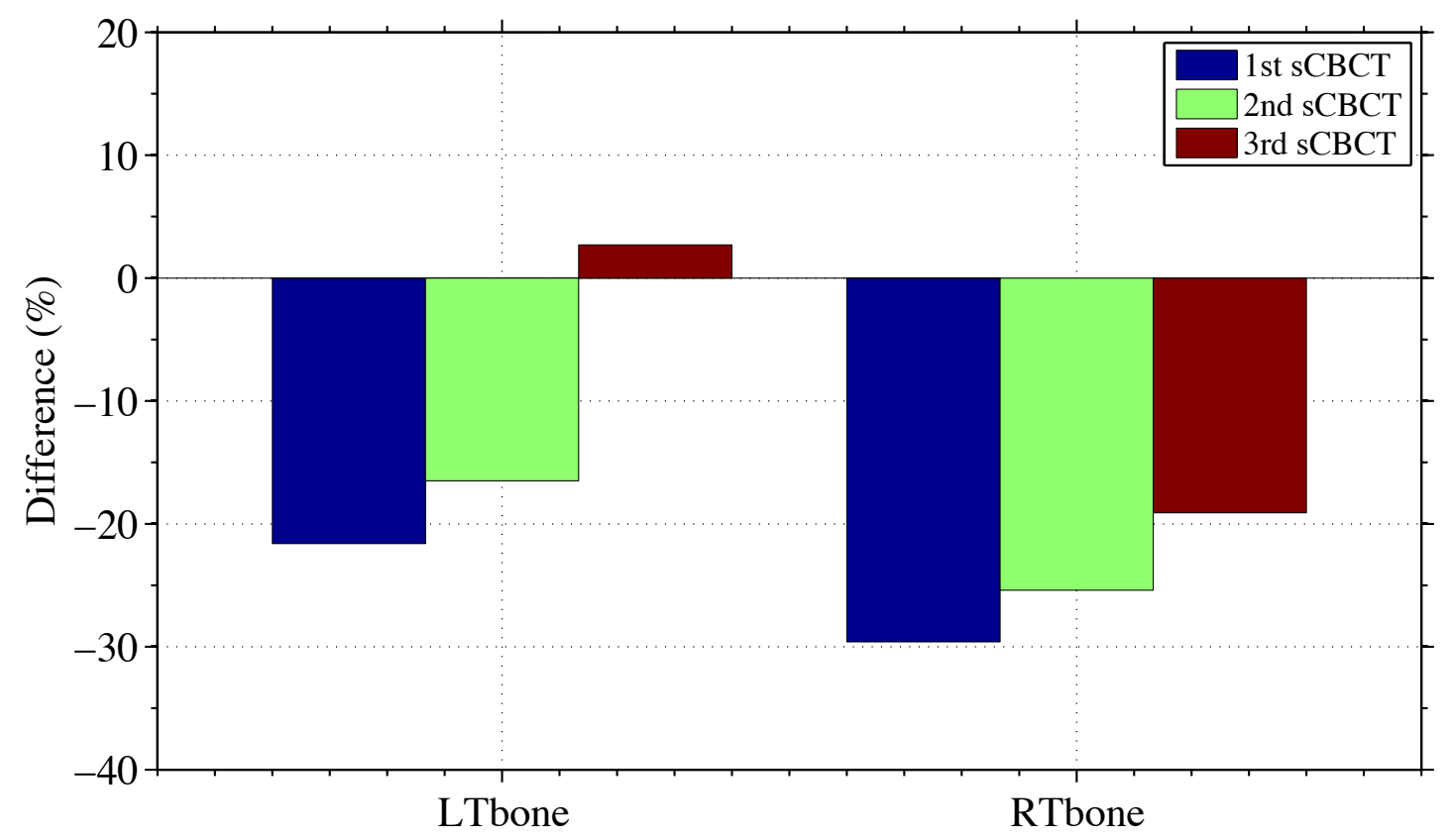

Figure 9.4: Right/Left hip and bone volume differences between $p C T$ and $1^{s t} s C B C T, 2^{\text {nd }}$ $s C B C T$ and $3^{\text {rd }} s C B C T$.

Figure 9.4 shows the differences in the LT and RT bone volumes between the pCT scan, $1^{\text {st }}$ sCBCT, $2^{\text {nd }}$ sCBCT and $3^{\text {rd }}$ sCBCT scans. Compared with the pCT scan, the $1^{\text {st }} \mathrm{sCBCT}$ scan showed the largest differences in the LT and RT bone volumes which were underestimated by $-21.6 \%$ and $-29.6 \%$, respectively. On the other hand, the smallest differences in the LT and RT bone volumes between the pCT and SCBCT was achieved by the $3^{\text {rd }}$ sCBCT scan where the differences were $2.7 \%$ and $-19.1 \%$, respectively. Therefore, the $3^{\text {rd }} \mathrm{sCBCT}$ scan is considered to be the most accurate representation of the bony structures compared with the pCT scan. Besides the accuracy of the MLT algorithm, another reason for the underestimation of both bone volumes in the three sCBCT scans might be the fact that streak artefacts in the pCT increased the number of high HU values and these were not corrected for (only for dose calculation). In the sCBCT, the MLT algorithms attempted to correct for this.

\subsubsection{Gamma and conformity index comparison}

Figure 9.5 (a) shows the $\gamma$ index evaluation for the calculation points falling inside the PTV, rectum and bladder for different bins, showing the fraction of points resulting with $\gamma<1$. In general, going from the $1^{\text {st }}$ sCBCT to the $3^{\text {rd }}$ sCBCT plans, the number of calculation points which passed $(\gamma<1)$ decreased, except for the PTV and bladder 

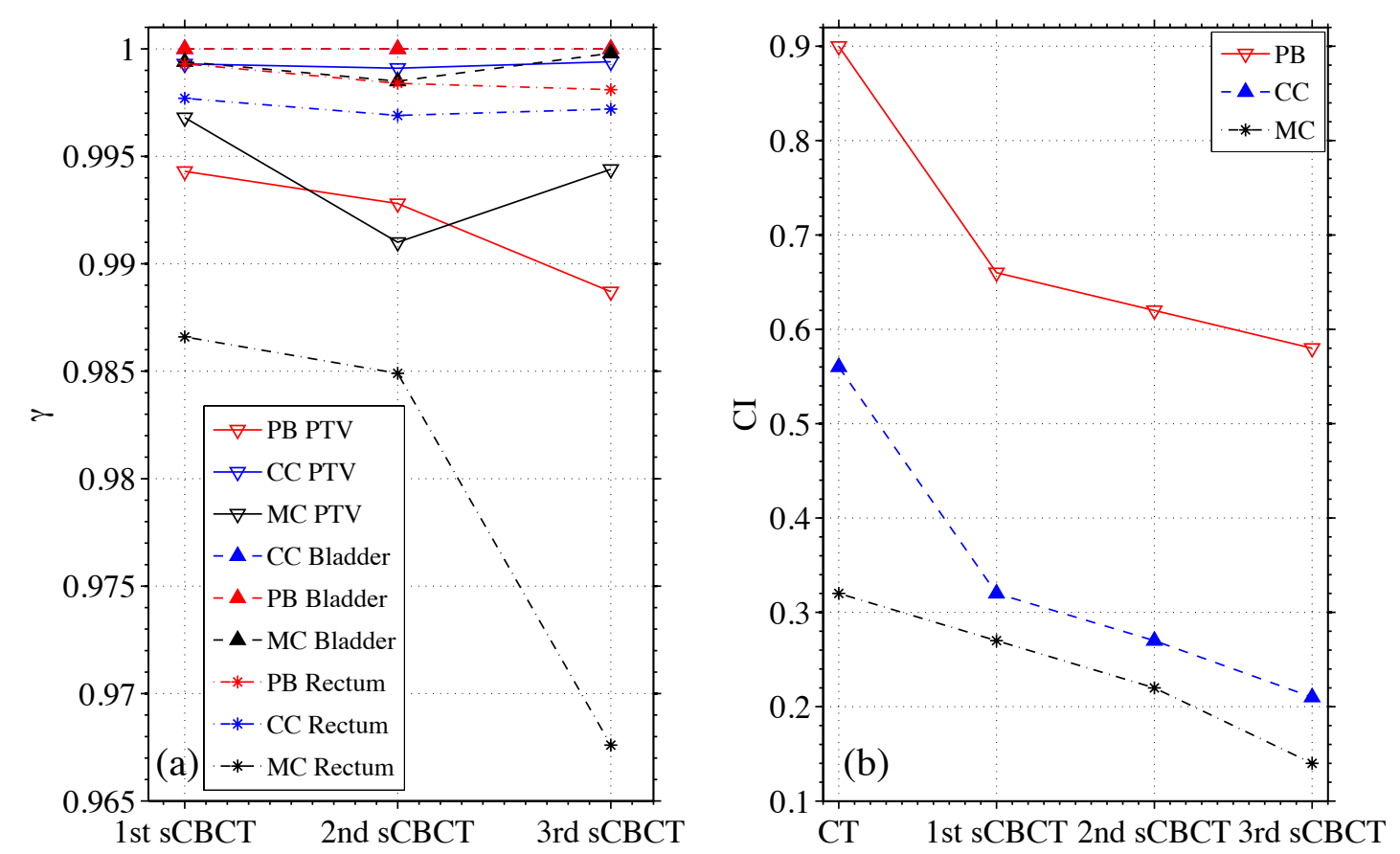

Figure 9.5: (a) Summary of the $\gamma$ index with fixed DTA $=3 \mathrm{~mm}$ and $D D=3 \%$ for the calculation points falling inside the PTV, rectum and bladder, showing the fraction of points resulting with $\gamma<1$. (b) CI comparison between $p C T$ and $s C B C T$ plans using PB, CC and MC algorithms.

region when using $\mathrm{CC}$ and $\mathrm{MC}$ algorithms, where some fluctuation was observed. Even with the presences of such a fluctuation, the fraction of the number of the calculation points that showed $\gamma<1$, in the three sCBCT plans, is still considered to be clinically acceptable (Son et al., 2015).

Figure 9.5 (b) shows the CI values of the pCT, $1^{\text {st }} \mathrm{sCBCT}, 2^{\text {nd }} \mathrm{sCBCT}$ and $3^{\text {rd }}$ sCBCT plans using PB, CC and MC algorithms. It can be clearly seen that the CI value, compared with the $\mathrm{pCT}$, dropped almost linearly from the $1^{\text {st }} \mathrm{sCBCT}$ to the $3^{\text {rd }}$ sCBCT plan. This shows that as the sCBCT represents bone volumes that are more closer to those in the pCT, the differences in the $\mathrm{CI}$ values increased. Then the difference would be expected to be constant, reaching a steady level. This represents the true difference between the $\mathrm{pCT}$ and $\mathrm{CBCT}$ scans due to the actual anatomical difference. Therefore, that level can be considered as the ground truth. In addition, the result also shows that the gradients of $\mathrm{CC}$ and $\mathrm{MC}$ lines are slightly steeper than the gradient of the $\mathrm{PB}$ line showing that $\mathrm{CC}$ and $\mathrm{MC}$ algorithms are more sensitive to the changes in the bone volumes than the PB algorithm (see Section 8.3.2). 


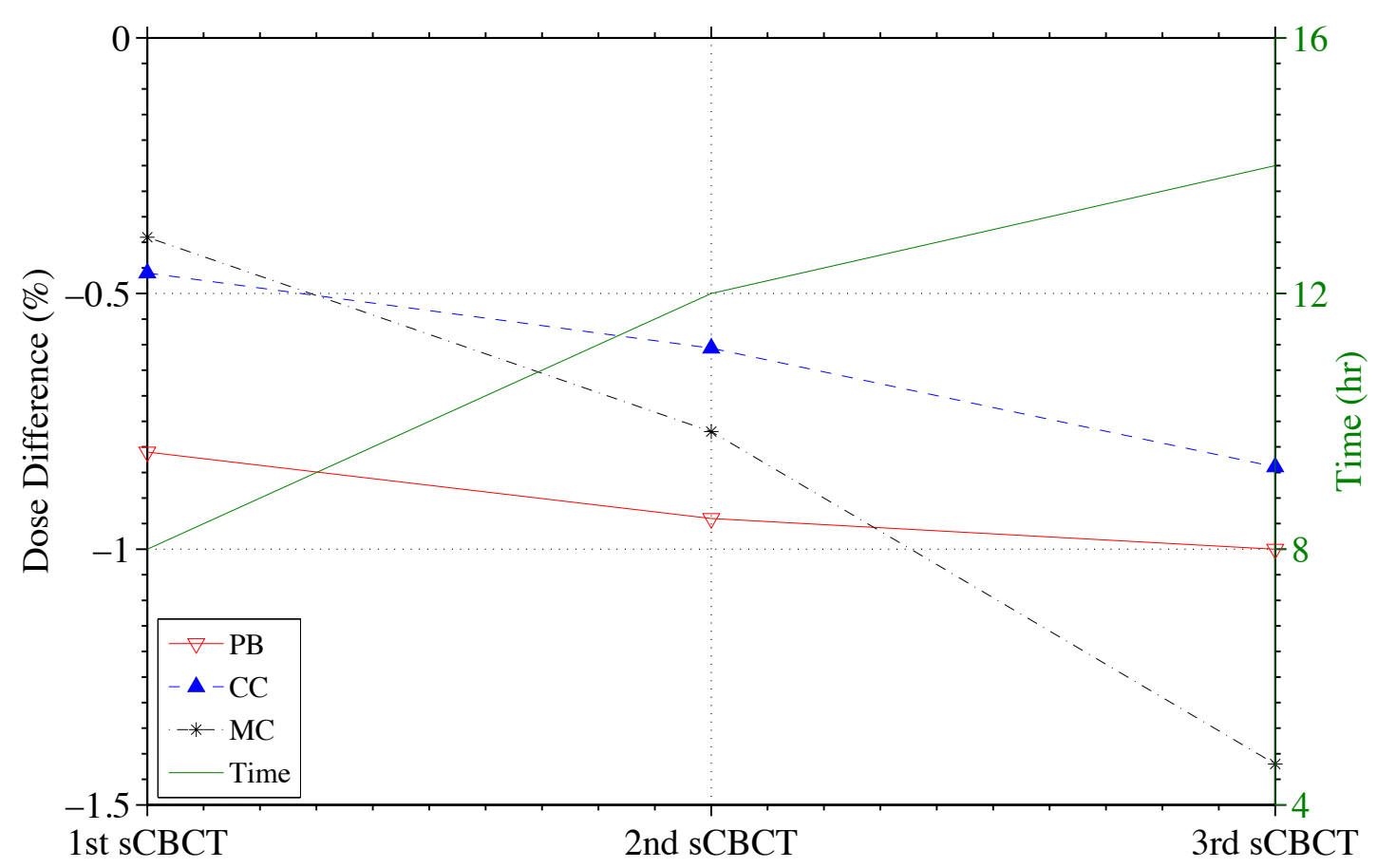

Figure 9.6: Dose comparison between $p C T$ and sCBCT plans at the isocentre against operator time using $P B, C C$ and $M C$ algorithms.

\subsubsection{Dose point comparison}

Figure 9.6 shows the dose difference between $\mathrm{pCT}$ and the $1^{\text {st }} \mathrm{sCBCT}, 2^{\text {nd }} \mathrm{sCBCT}$ and $3^{\text {rd }} \mathrm{SCBCT}$ plans at the isocentre using all algorithms plotted against the operator time needed to segment each sCBCT. Based on the local department condition for replanning (see Section 8.2.5.3), all three sCBCT plans passed the criteria, showing differences of less than $-2 \%$ compared with the pCT plan when using all algorithms. From the $2^{\text {nd }} \mathrm{sCBCT}$ to the $3^{\text {rd }} \mathrm{sCBCT}$, it required $50 \%$ more operator time to improve the accuracy by $0.07 \%, 0.23 \%$ and $0.65 \%$ when using $\mathrm{PB}, \mathrm{CC}$ and $\mathrm{MC}$ algorithms, respectively. For $1^{\text {st }} \mathrm{sCBCT}$, compared with $2^{\text {nd }} \mathrm{sCBCT}$, it required $50 \%$ less time to provide dose differences of $-0.81 \%,-0.46 \%$ and $-0.39 \%$ when using PB, CC and MC algorithms, respectively. Such a difference is considered to be clinically acceptable if the $\mathrm{pCT}$ is the ground truth for dose calculation. Therefore, the $1^{\text {st }} \mathrm{sCBCT}$ scan is considered to provide the optimal level which balances the accuracy of the calculation and the time required ( 8 hours). 


\subsection{Conclusion}

The segmentation of CBCT images in multiple hip prostheses using the MLT method in this study can be used for dose calculation. In this chapter, the segmentation of CBCT of a prostate patient with bilateral hip prostheses was investigated. Based on the result of the previous chapter, the CBCT images were segmented into five values of HU (air, adipose, water, bone and metal implant) in three different sets, where the only difference was about the bone volume. The operator time for segmenting the $1^{\text {st }}$ sCBCT, $2^{\text {nd }} \mathrm{sCBCT}$ and $3^{\text {rd }} \mathrm{sCBCT}$ was 8,12 and 14 hours, respectively. The results showed that as the operator time to segment each set increased the differences in the bone volumes decreased compared with the pCT scan. However, as the differences in the bone volumes decreased the dose difference increased between the sCBCT and pCT plans. Nevertheless, the dose difference is considered to be acceptable if the pCT scan was considered as the ground truth for dose calculation. Thus, as a result, $1^{\text {st }}$ sCBCT scan, even though it showed the largest difference in the bone volumes compared with the pCT scan, gave the best balance between the dose accuracy ($0.8 \%$ ) and operator time ( 8 hours). This could be implemented in the clinic as a decision-making tool for re-planning where a clinical decision could be made in the time between treatment fractions. The next step is to develop an automated MLT algorithm to reduce the operator time associated with the MLT algorithm. 
Chapter 10 Automated algorithm for CBCT-based dose calculations of a prostate radiotherapy 


\subsection{Introduction}

In Chapter 8, it has been shown that segmenting the CBCT images of a prostate patient with a single hip prosthesis into five material bins using the MLT algorithm, resulted in a dose accuracy $(-1.9 \%)$ that is clinically acceptable as an on-treatment assessment for changes in internal anatomy, shape and size. For a prostate patient with double hip prostheses, the segmentation using the same method achieved a dose accuracy of $-0.8 \%$, as shown in Chapter 9 . The operator time needed to segment the CBCT images was 3.5 and 8 hours, respectively. This is because it was necessary to extend the MLT algorithm to categorize pixel values on a region-by-region basis, with the operator time depending on image artefacts as well as the additional difficulties presented by the metallic hip prosthesis. However, the length of operator time needed could make it difficult to implement this as a technique in the clinic as the main reason of proposing this method is to use it as a quick-decision-making tool for replanning. This will eliminate the need to rescan the patient in pCT and potentially avoid an additional dose. The aim of this chapter, therefore, was to reduce the operator time associated with the MLT algorithm by developing an automated MLT algorithm, and test its accuracy on patient cases that were already used in this thesis.

\subsection{Methodology}

\subsubsection{CT and CBCT image acquisition}

The CT and CBCT images used in this chapter are the same images as those used in Chapters 7, 8 and 9, of standard (without metal implant), single and double hip replacement prostate patients.

\subsubsection{Modification of CBCT image}

In this chapter, the MLT algorithm was used in two ways: using a manual and an automated procedure. In the manual procedure, the CBCT images were segmented using the same method previously described (see Chapter 7, 8 and 9). The resultant segmented CBCT images using this procedure are referred to as $\mathrm{SCBCT}_{\operatorname{man}}$.

In the automated procedure, the CBCT images of each case were divided into five concentric rings, which are uniform in shape through all slices, using MATLAB 


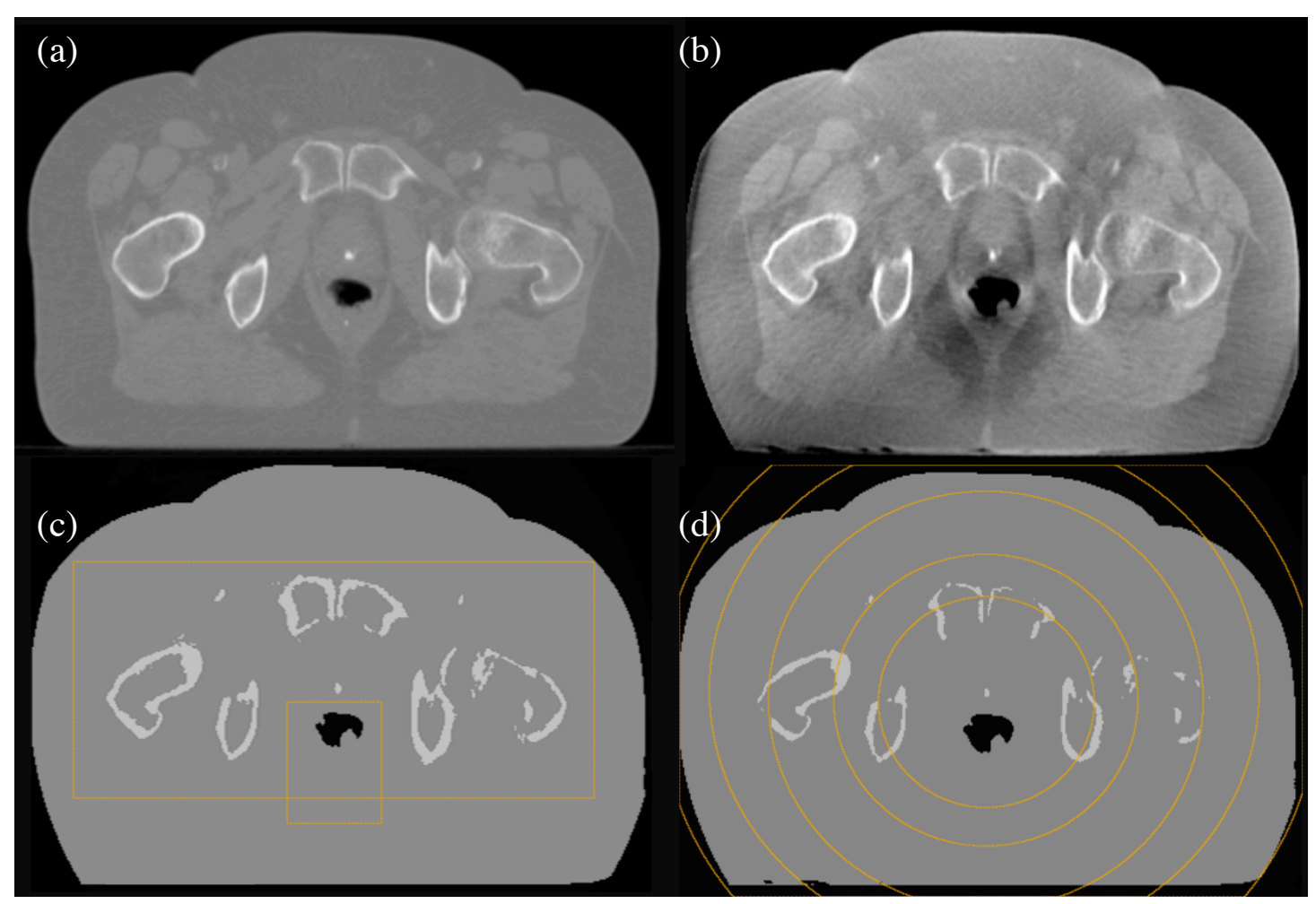

Figure 10.1: A slice of the $p C T(a)$ of the standard case and the original CBCT (b), and the resultant images after segmentation CBCT using the manual MLT (sCBCT man) and the automated MLT ( $s C B C T_{\text {auto }}$ ) ( $c$ and d respectively).

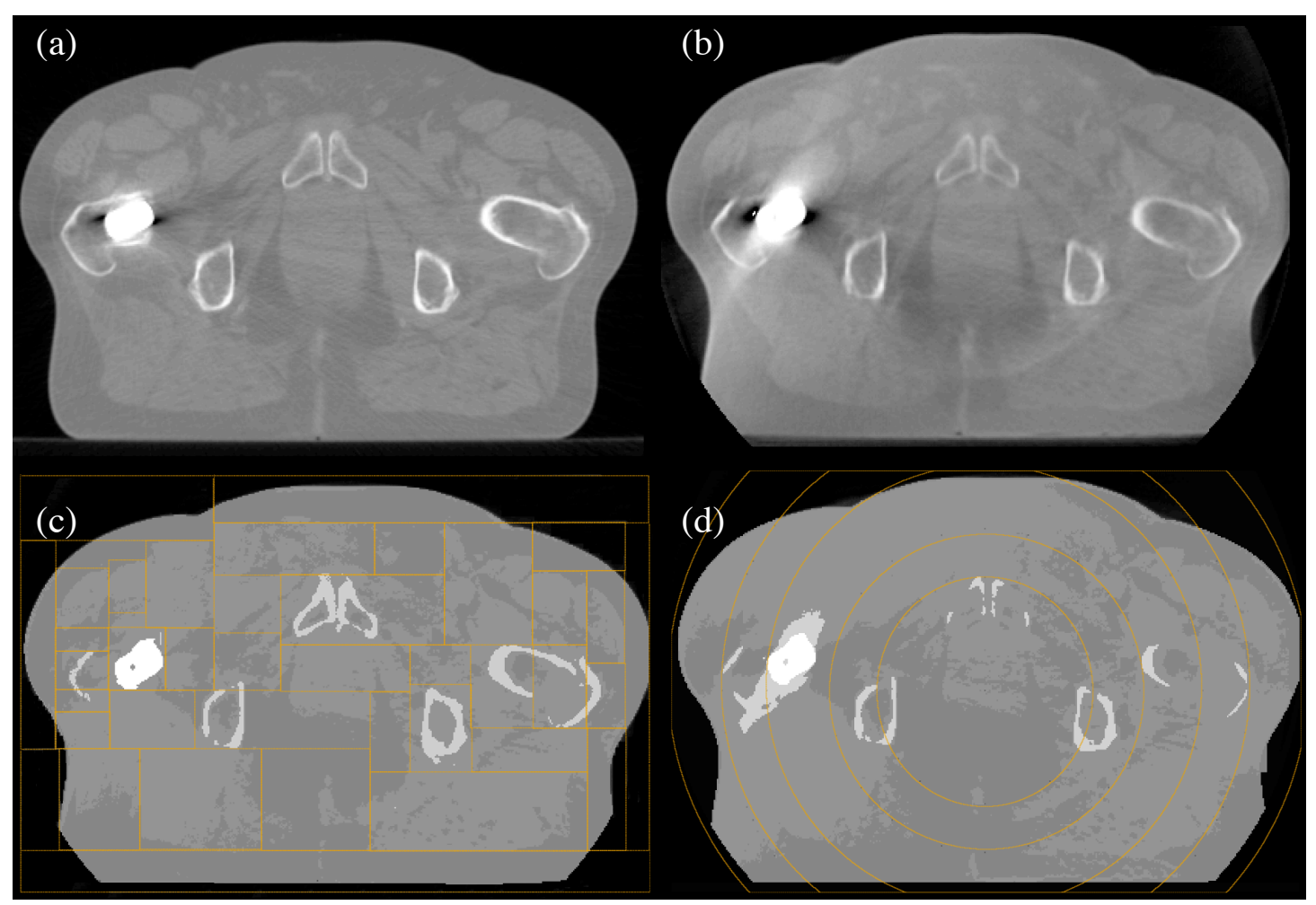

Figure 10.2: A slice of the $p C T$ (a) of the single hip prosthesis case and the original CBCT (b), and the resultant images after segmentation CBCT using the manual MLT (sCBCT man) and the automated MLT ( $S C B C T_{\text {auto }}$ ) (c and d respectively). 


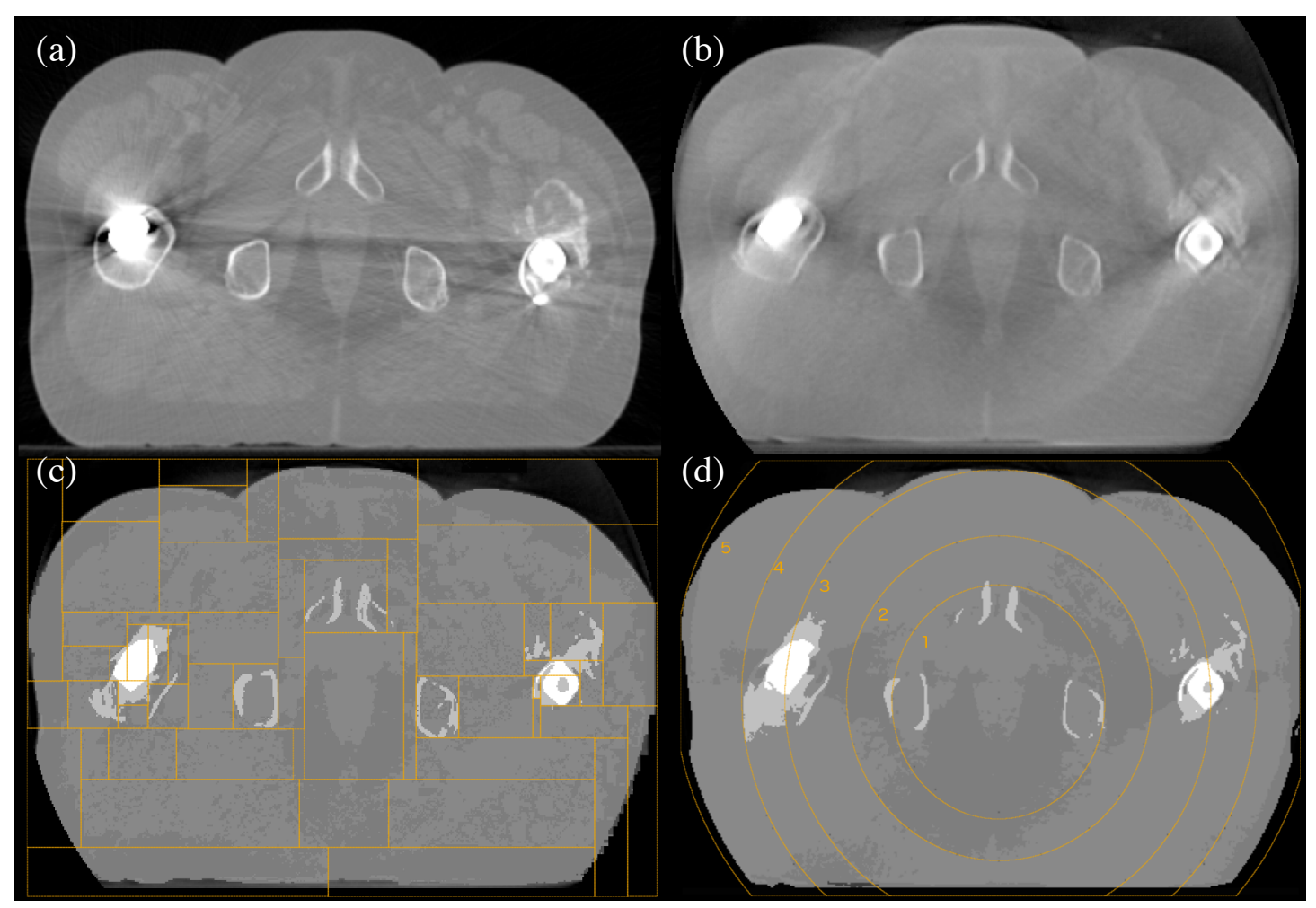

Figure 10.3: A slice of the $p C T(a)$ of the bilateral hip prostheses case and the original $C B C T$ (b), and the resultant images after segmentation CBCT using the manual MLT ( $S C B C T_{\text {man }}$ ) and the automated MLT ( $S C B C T_{\text {auto }}$ ) ( $c$ and d respectively).

scripts, as shown in Figure 10.1(d), 10.2(d) and 10.3(d). The centre of the inner radius of each case was defined as the centre of the patient geometry, which can be changed by the user. The lower threshold values for each material changes with the radius and slice as the image/pixel histogram of any radius changes from slice to slice. Figure 10.4(a) shows the image histograms (56 curves) of the bilateral hip prostheses in radius number 4 only, for 56 slices. It can be seen that the histogram of some slices are broader than other, showing that the metal and other CBCT artefacts caused by the presence of the two hip prostheses in these slices spread out the pixel values. There are two fixed peaks that only change in magnitude. These peaks represent air and water, but in other radii, there would be only one peak that represents water as there is no air region. However, the threshold values can be easily determined by the user's analysis of the central slice. For example, the lower threshold value for water, in the inner radius, was defined in relation to the pixel value with the maximum frequency in the slice according to the ratio of the lower threshold value of water and the pixel value with the maximum frequency in the central slice (Figure 10.4(b)). In this example, the central slice is, usually, the main PTV slice and has a pixel value with 

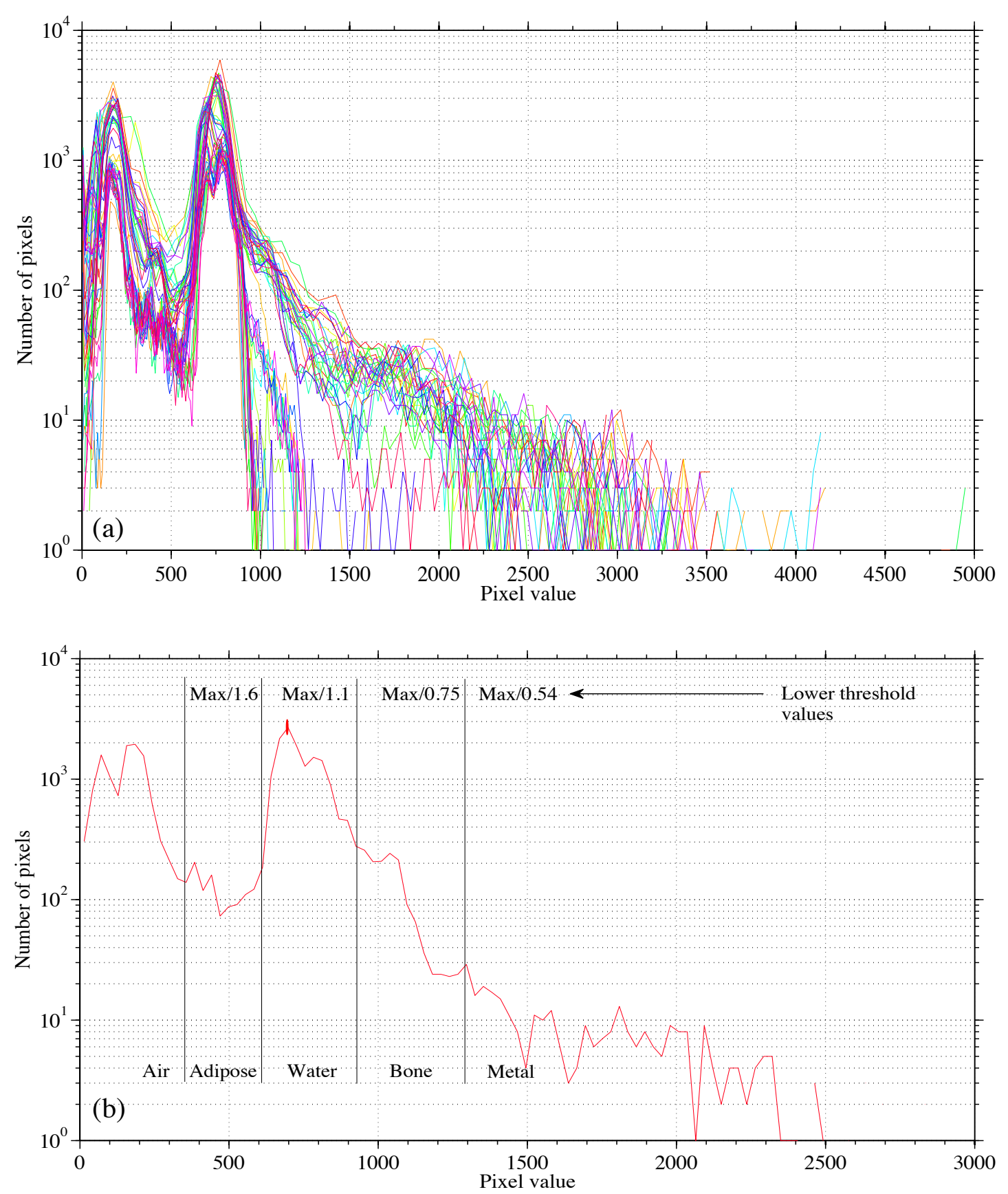

Figure 10.4: Image histogram of the bilateral hip prostheses case in radius number 4 only through all slices (a) and an image histogram of a single slice in radius number $4(b)$, showing how the lower threshold values of each material are defined.

the maximum frequency of 700 in radius number 4 . By visualising the radius number 4 in the central slice, the user can then select the lower threshold value for water which is, in this case, approximately 635. For radius number 4 only, the lower threshold value for water in any other slice is then the pixel value with maximum frequency divided by 1.1 which is the result of the division of 700/635 (Figure 10.4(b)). Therefore, the variation in the pixel value within this radius in each slice is 
taken into account when defining the lower threshold of water. The same procedure was applied for each material bin in each radius. Thus the threshold values are defined automatically and it takes approximately 20 minutes of operator time. The selection of the centre of the inner radius, as well as the selection of the lower threshold value for each material in each radius in the central slice, is considered in the operator time. The resultant segmented $\mathrm{CBCT}$ images using this procedure are referred to as $\mathrm{sCBCT}_{\text {auto }}$.

The motivation for using a radial shape was the fact that, in CBCT, the issue of the scatter occurs spherically and ring artefacts that caused by miscalibrated detector pixel lines/rows, elements or manufacturing defects at a fixed location in the FPD. In addition, due to the presence of the hip prostheses, the low energetic X-rays are absorbed, thus the polychromatic beam becomes gradually harder. Consequently, the FPD exhibits pixel-to-pixel sensitivity variations, which lead to ring artefacts. In a pelvic region with prostheses, there is a rapid change in the exposure to the FPD from frame to frame, fluctuating between high exposure and by low exposure due to the strong attenuation of the metal. This leads to radar artefacts that appear as a circular radar bright-shaded region, owing to inconsistencies in the detector signal and/or gain (see Section 2.5). However, based on the results of Chapter 7, the CBCT images of the standard were segmented into three material bins using the automated MLT algorithms. For the single and double hip prostate cases, the CBCT images were segmented into five material bins (see Chapter 8 and 9).

\subsubsection{OMP-based treatment plan}

The same IMRT treatment plan parameters, shown in tables 7.1, 8.2 and 9.1 for the standard, single hip and double hip prostheses patients, respectively, were performed on the $\mathrm{sCBCT}_{\text {auto }}$ images (see Chapter 7,8 and 9).

\subsubsection{MC-based treatment plan}

In the previous chapters mentioned above, all the input files, for each BEAMnrc and DOSXYZnrc, were created manually for each segment of each IMRT plan (total of 28, 30 and 23 segments for the standard, single hip and double hip prostheses case, respectively). It is time consuming to write an input file for BEAMnrc and another 
one for DOXYZnrc for each segment and these are also subject to errors. In this chapter, an automated procedure was developed and applied to the DICOM-RT file to extract the treatment plan parameters, using a MATLAB script, and then convert and write them in a MC-format input file (egsinp file) for BEAMnrc and DOSXYZnrc, separately. Applying the script to the DICOM-RT file eliminated the need to use the in-house Excel macro (written by Mark Edwards) that had been used previously to get the treatment plan parameters. These parameters include collimators positions, backup jaws positions, secondary jaws positions, isocentre coordinates, SSD, gantry angle and collimator angle. The coordinate difference between the OMP and MC system is taken into account. The script also automatically sets the random number generator (RNG) seeds to different values in each input file to avoid potential correlations in pseudo-random sampling. In addition, the script is an interactive script that requires the number of histories and the input file name, for each BEAMnrc and DOSXYZnrc, and the directory and the name of the egsphant file. Finally, the outputs from the script are the input files with names that are provided by the user with beam number and the segment number of that beam, i.e. prostate1_5.egsinp, where 1 is the beam number and 5 is the segment number.

The simulation or calculation of each segment was performed on the egsphant file, which was generated from $\mathrm{sCBCT}_{\text {auto }}$ images (see Section 6.2.6). Then the same steps described in Chapter 7,8 and 9 were used in this chapter.

\subsubsection{Treatment planning evaluation and comparison}

For each case, the $\mathrm{sCBCT}_{\text {auto }}$ and $\mathrm{pCT}$ images were fused using ProSoma software and the structure sets were then transferred to the $\mathrm{sCBCT}_{\text {auto }}$ images without any modification except for the external contour, where there are some differences. The plans were then copied to $\mathrm{SCBCT}_{\text {auto }}$ using the same geometry and $\mathrm{MU}$ values and the doses were recalculated using $\mathrm{PB}, \mathrm{CC}$ and $\mathrm{MC}$ algorithms. These steps were already performed for $\mathrm{sCBCT}_{\operatorname{man}}$ in previous chapters. For the single hip prosthesis case, the sCBCT5 scan was used and referred to as the $\mathrm{sCBCT}_{\operatorname{man}}$. For the double hip prostheses case comparison, the $1^{\text {st }} \mathrm{SCBCT}$ scan only was used and referred to as the $\mathrm{sCBCT}_{\operatorname{man}}$. 


\subsubsection{Dose profile comparison}

The dose profiles around the reference point (isocentre) depth were compared and plotted against the $\mathrm{CT}, \mathrm{sCBCT}_{\text {man }}$ and $\mathrm{sCBCT}_{\text {auto }}$ numbers to show how close the $\mathrm{sCBCT}_{\operatorname{man}}$ and $\mathrm{sCBCT}_{\text {auto }}$ number profiles are close to the reference $\mathrm{CT}$ number at that depth using MC algorithm. The absolute differences between the $\mathrm{pCT}, \mathrm{sCBCT}_{\operatorname{man}}$ and $\mathrm{sCBCT}_{\text {auto }}$ dose profiles were plotted to aid comparison. The dose profile comparison was performed using only the MC algorithm. In addition, the maximum dose, mean dose and minimum dose parameters for PTV, rectum and bladder were compared between the $\mathrm{pCT}$ plans and $\mathrm{sCBCT}_{\operatorname{man}} / \mathrm{sCBCT}_{\text {auto }}$ plans.

\subsubsection{Hip and bone volume comparison}

The volumes of right (RT)/left (LT) bone were calculated in the pCT scan and compared with those in the $\mathrm{sCBCT}_{\operatorname{man}}$ and $\mathrm{sCBCT}_{\text {auto }}$ scan for the standard case. For the single and double hip prostheses cases, the volume of right/left hip and bone were compared. Such a comparison was performed to show how close the two scans were to the pCT scan.

\subsubsection{Gamma and conformity index comparison}

For the three cases, gamma index analysis was performed using the pCT plan as a reference in each, in the PTV, rectum and bladder structures. The same criteria used in the previous chapters were used. In addition, the CI was calculated for $\mathrm{sCBCT}_{\text {auto }}$ plans and then compared with the pCT plans using $\mathrm{PB}, \mathrm{CC}$ and $\mathrm{MC}$ algorithms.

\subsubsection{Dose point comparison}

The dose at the isocentre (at the geometric centre of the prostate PTV) was compared between the $\mathrm{pCT}$ and $\mathrm{sCBCT}_{\mathrm{man}} / \mathrm{sCBCT}_{\text {auto }}$ plans. 


\subsection{Result and discussion}

\subsubsection{Dose profile comparison}

Figure 10.5 shows the cross-profile of $\mathrm{pCT}, \mathrm{sCBCT}_{\operatorname{man}}$ and $\mathrm{sCBCT}_{\text {auto }}$ around the isocentre depth as well as the $\mathrm{pCT}, \mathrm{sCBCT}_{\operatorname{man}}$ and $\mathrm{sCBCT}_{\text {auto }}$ numbers at that depth for (a) the standard, (b) single hip and (c) double hip prostheses cases using MC algorithms. For the standard case, the $\mathrm{sCBCT}_{\operatorname{man}}$ and $\mathrm{sCBCT}_{\text {auto }}$ number profiles are in good agreement with the $\mathrm{pCT}$ number profile, especially at the bone/tissue interfaces. In some bone regions, the $\mathrm{sCBCT}_{\text {auto }}$ numbers showed less agreement with

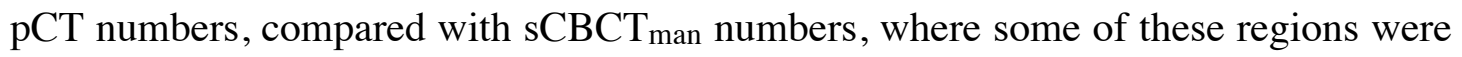
considered as water. However, in general, the dose differences between $\mathrm{sCBCT}_{\text {auto }}$ and $\mathrm{pCT}$ profiles were less compared with the dose differences for $\mathrm{sCBCT}_{\operatorname{man}}$ and pCT profiles.

For the single hip prosthesis case, the $\mathrm{sCBCT}_{\operatorname{man}}$ and $\mathrm{sCBCT}_{\text {auto }}$ number profiles are in good agreement with the pCT number profile, especially at the implant/tissue interface. It can be clearly seen that the pCT number increased gradually at the implant/tissue interface until reaching the maximum pCT number, which is 4000 , representing the hip metallic. This is due to the partial volumes effect in that region. In addition, one notable feature in the pCT number profile is the sharp drop before and after the hip prosthesis region, due to the metal and zero-data artefacts presented in the pCT images. The $\mathrm{sCBCT}_{\operatorname{man}}$ and $\mathrm{sCBCT}_{\text {auto }}$ number profiles both showed a highly sharp increase in the scan number, where it jumped from the water value, 1024 , to the hip metallic value, 4000. This is due to the fact that the partial volumes effect in the sCBCT scans was considered either water or metal, due to the nature of the threshold method. In addition, the metal and zero-data artefacts, which also appeared in the original CBCT images, were replaced with the metal CT/HU value in

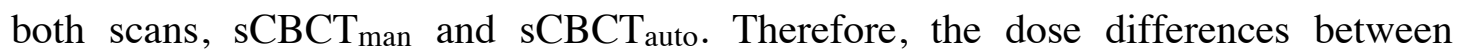
$\mathrm{sCBCT}_{\mathrm{man}} / \mathrm{sCBCT}_{\text {auto }}$ and $\mathrm{pCT}$ profiles were higher in that region. However, the $\mathrm{sCBCT}_{\text {auto }}$ number/dose profiles are comparable to those of $\mathrm{sCBCT}_{\text {man }}$ with much less (approximately 90\%) operator time.

For the double hip prostheses, the $\mathrm{sCBCT}_{\operatorname{man}}$ and $\mathrm{sCBCT}_{\text {auto }}$ profiles are in good agreement with the pCT profile, especially at the implant/tissue interface. For bone 

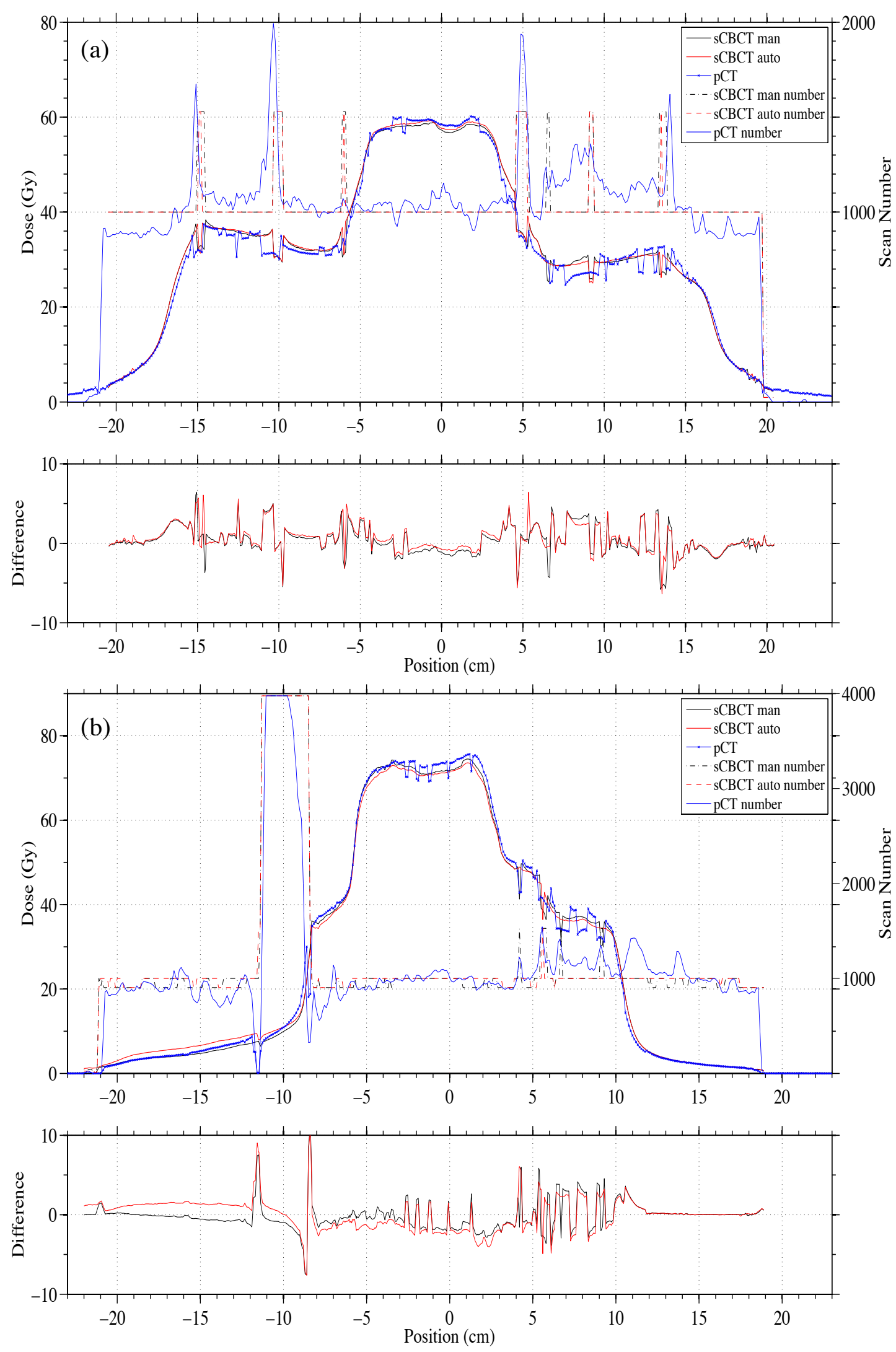

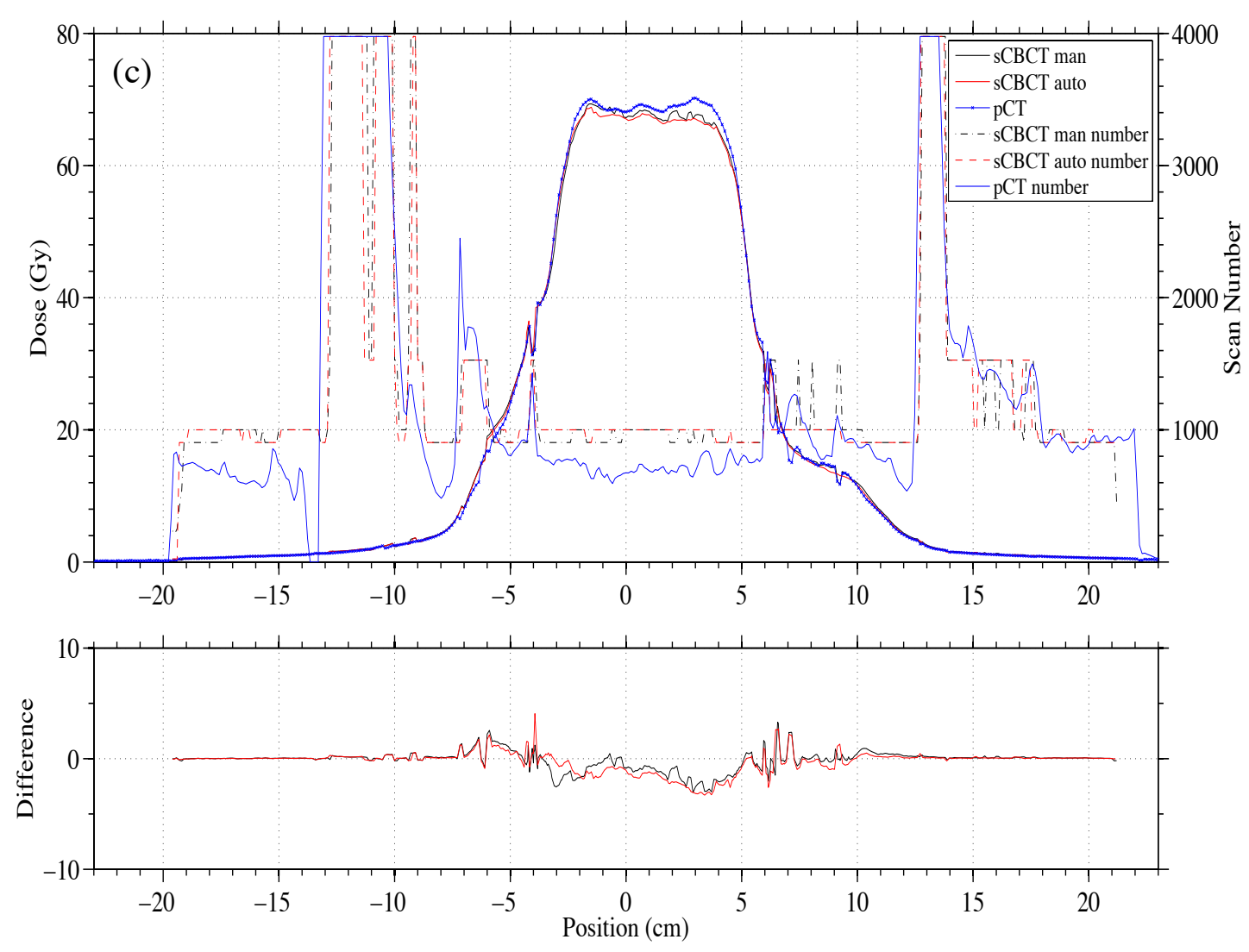

Figure 10.5: Comparison of the dose profiles of $p C T, s C B C T_{\text {man }}$ and $s C B C T_{\text {auto }}$ plans around the isocentre depth using MC algorithm for the (a) standard, (b) single hip and (c) double hip prostheses cases. The second y axis represents the $s C B C T_{m a n}$ number, $s C B C T_{\text {auto }}$ number and $p C T$ number. Subplots represent the differences between $p C T$ and $s C B C T_{\text {man }} / s C B C T_{\text {auto }}$ dose profiles.

regions, the $\mathrm{sCBCT}_{\text {auto }}$ numbers showed less agreement with the $\mathrm{pCT}$ numbers, compared with the $\mathrm{sCBCT}_{\text {man }}$ numbers, where some of these regions were considered as water. In addition, the $\mathrm{sCBCT}_{\text {auto }}$ overestimated some adipose tissue regions and considered it as water, especially in the PTV region (high-dose region), leading to an underestimation of the dose in that region by $-4.4 \%$. The $\mathrm{sCBCT}_{\text {man }}$ numbers suggested there was more adipose tissue than the $\mathrm{sCBCT}_{\text {auto }}$ numbers, thus the dose difference with the $\mathrm{pCT}$ dose profile was less when compared with the $\mathrm{sCBCT}_{\text {auto }}$ dose profile. Similar to the single prosthetic hip case, there were sharp drops in the pCT number around the metals due to the metal artefacts presented in the pCT images, whereas in $\mathrm{sCBCT}_{\operatorname{man}}$ and $\mathrm{sCBCT}_{\text {auto }}$, these were replaced with the adipose and water $\mathrm{CT} / \mathrm{HU}$ values. However, the $\mathrm{sCBCT}_{\text {auto }}$ number/dose profiles are comparable to those of $\mathrm{sCBCT}_{\operatorname{man}}$ with much less (approximately $95 \%$ ) operator time. 
Tables 10.1, 10.2 and 10.3 show the dose differences between the $\mathrm{sCBCT}_{\text {man }}$ and $\mathrm{sCBCT}_{\text {auto }}$ plans and the pCT plans for the standard, single hip and double hip prostheses, respectively, for the PTV, rectum and bladder. Although there are differences in material assignments (manual and automated MLT algorithms), the overall difference in the calculated dose was small. In general, for the three cases the $\mathrm{sCBCT}_{\text {auto }}$ plans showed similar dose differences with the $\mathrm{pCT}$ plans compared to the $\mathrm{sCBCT}_{\text {man }}$ plans, and it even showed better dose accuracy in the standard case. This suggested that the automated MLT algorithm works better than the manual MLT if there are no additional inhomogeneities in the patient anatomy.

Table 10.1: Dose and coverage differences between sCBCT plans and $p C T$ plan, in $\%$, for the $P T V$, rectum and bladder for the standard prostate case.

\begin{tabular}{|c|c|c|c|c|c|c|c|}
\hline & & \multicolumn{3}{|c|}{$\mathrm{sCBCT}_{\text {man }}$} & \multicolumn{3}{|c|}{$\mathbf{s C B C T}_{\text {auto }}$} \\
\hline & & PB & $\mathrm{CC}$ & MC & PB & $\mathrm{CC}$ & MC \\
\hline \multirow{3}{*}{ PTV } & $\mathbf{D}_{\max }$ & -0.96 & -1.63 & 0 & -0.64 & -1.3 & -2.2 \\
\hline & $\mathbf{D}_{\text {mean }}$ & -0.76 & -0.89 & -1.58 & -1.8 & -0.47 & -1.1 \\
\hline & $\mathbf{D}_{\min }$ & -2.12 & 1.91 & 61.64 & -17.01 & 1.53 & 3.5 \\
\hline \multirow{3}{*}{ Rectum } & $\mathbf{D}_{\max }$ & -0.97 & -0.67 & -3.62 & -0.64 & -1 & -4.28 \\
\hline & $\mathbf{D}_{\text {mean }}$ & -1.22 & -0.77 & 2.22 & -0.79 & -0.74 & 1.77 \\
\hline & $\mathbf{D}_{\min }$ & 0 & 0 & -11.76 & 0 & 0 & -11.76 \\
\hline \multirow{3}{*}{ Bladder } & $\mathbf{D}_{\max }$ & -1.29 & -1.31 & -3.23 & -0.64 & -0.65 & -2.58 \\
\hline & $\mathbf{D}_{\text {mean }}$ & -1.09 & -1.29 & -1.15 & -0.74 & -1.03 & -0.78 \\
\hline & $\mathbf{D}_{\min }$ & 0 & 0 & 6.45 & 0 & 0 & 6.45 \\
\hline
\end{tabular}


Table 10.2: Dose and coverage differences between sCBCT plans and $p C T$ plan, in \%, for the $P T V$, rectum and bladder for the single hip prosthesis case.

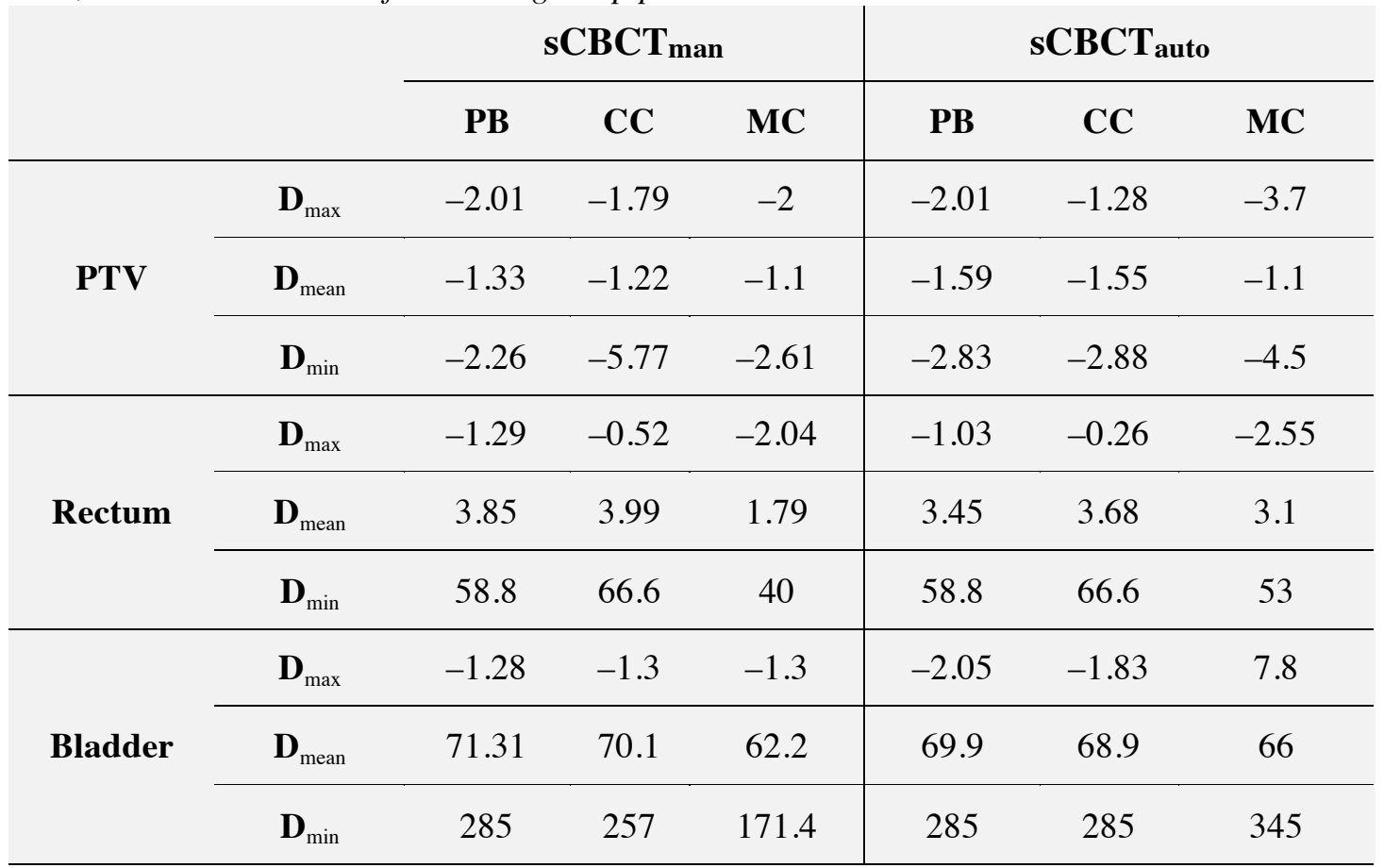

Table 10.3: Dose and coverage differences between sCBCT plans and $p C T$ plan, in $\%$, for the $P T V$, rectum and bladder for the double hip prostheses case.

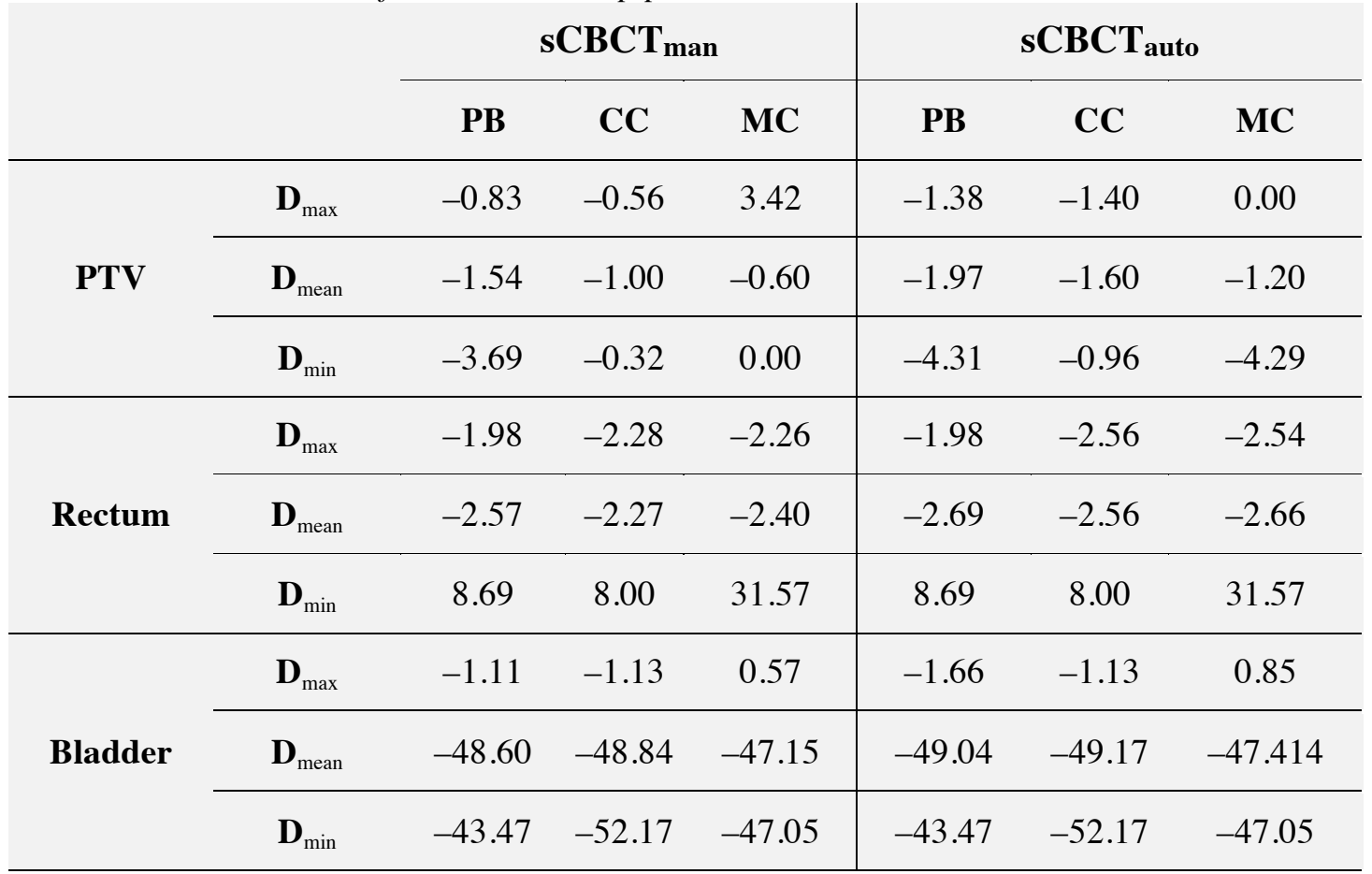




\subsubsection{Hip and bone volume comparison}

Figure 10.6 shows the differences in the LT/RT hip and bone volumes between the pCT scan and $\mathrm{sCBCT}_{\operatorname{man}} / \mathrm{sCBCT}_{\text {auto }}$ scans for the (a) standard, (b) single hip and (c) double hip prostheses cases. In general, using the manual MLT algorithm to segment CBCT images resulted in differences in the hip and bone volumes that were less than those when using the automated MLT algorithm, except for the RT hip in the single hip case and for the RT bone in the double hip prostheses case. Nevertheless, the differences in hip and bone volumes between $\mathrm{sCBCT}_{\operatorname{man}}$ and $\mathrm{sCBCT}_{\text {auto }}$ scans are comparable except for the LT bone in the standard and single hip prosthesis cases, and the LT hip in the double hip prostheses case. For the standard case, the MLT algorithm underestimates some bony regions and considered them as water. This underestimation may be the reason why the $\mathrm{SCBCT}_{\text {auto }}$ dose profile was closer to the pCT dose profile than the $\mathrm{sCBCT}_{\text {man }}$ dose profile, where the underestimation of the bony region was less pronounced (Figure 10.6(a)).

For the single hip prosthesis case, compared with the pCT scan the largest difference between the $\mathrm{sCBCT}_{\text {man }}$ and $\mathrm{sCBCT}_{\text {auto }}$ scans was found in the $\mathrm{LT}$ bone where it was underestimated by $-5 \%$ in $\mathrm{sCBCT}_{\text {man }}$ and by $-59 \%$ in $\mathrm{sCBCT}_{\text {auto }}$ (Figure 10.6(b)). This showed that the automated MLT algorithm was unable to correct scatter and beam hardening artefacts and erroneously replaced the artefacts with water $\mathrm{CT} / \mathrm{HU}$ values. By comparison, the manual MLT algorithm correctly replaced the artefacts with bone CT/HU values. For the RT hip, the automated MLT algorithm was able to correct metal artefacts, resulting in a volume difference with the pCT scan that was less compared with that of the manual MLT algorithm.

For the double hip prostheses case, the largest difference between $\mathrm{sCBCT}_{\operatorname{man}}$ and $\mathrm{sCBCT}_{\text {auto }}$ was found in the LT hip where in $\mathrm{sCBCT}_{\text {man }}$ it was overestimated by $6.8 \%$

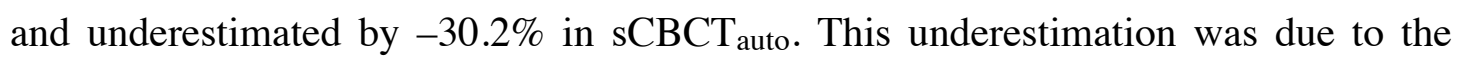
fact that the automated MLT algorithm was unable to accurately correct cupping artefacts due to the increased amount of scatter and beam hardening inside the LT hip, resulting in dark streaks. Thus, the automated MLT algorithm erroneously replaced the artefacts with bone HU values whilst the manual MLT correctly replaced the artefacts with metal HU values as shown in Figure 10.7. In fact, the automated MLT algorithm considered $43 \%$ of the LT hip volume as bone. Both MLT algorithms 

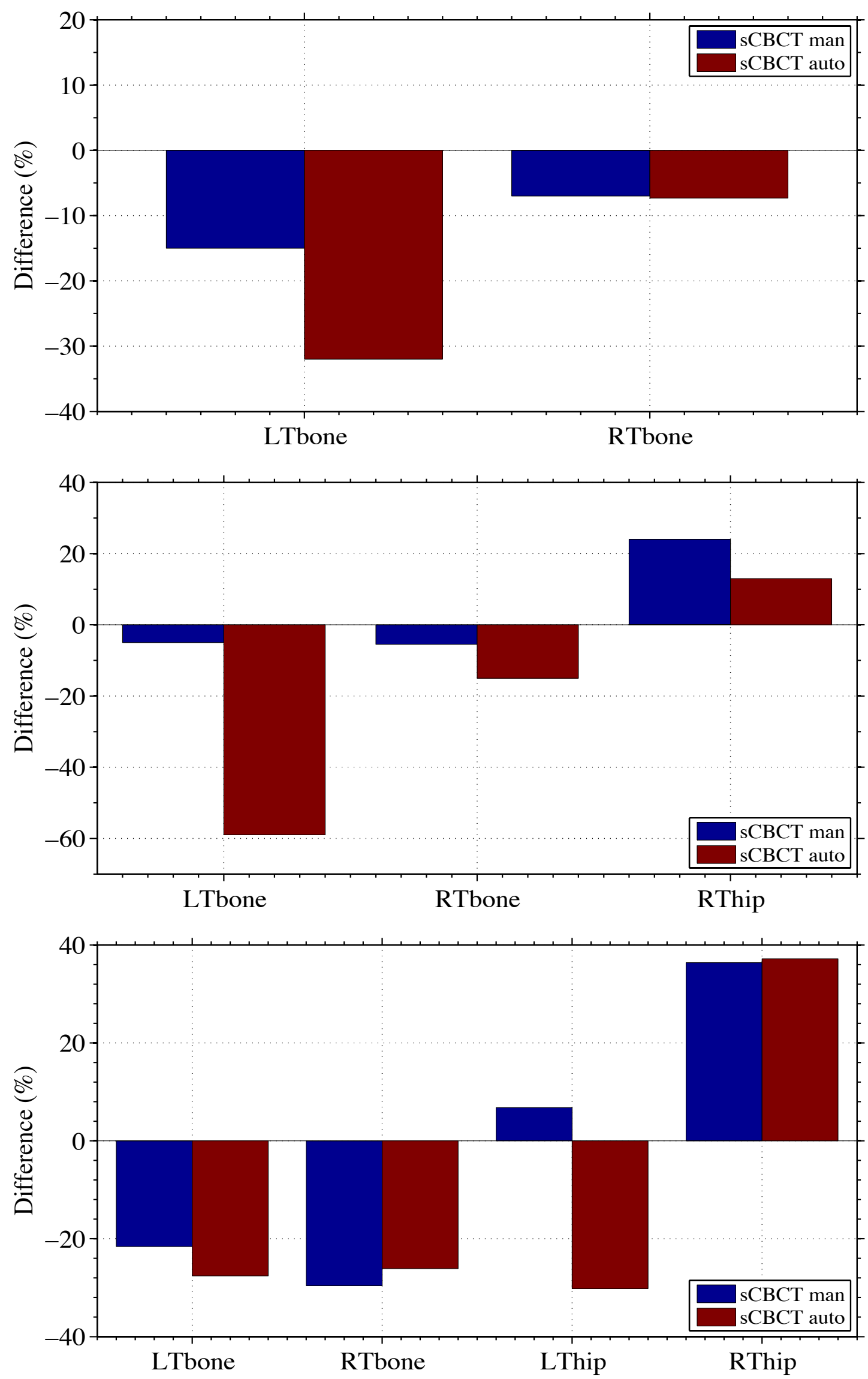

Figure 10.6: Right/Left hip and bone volume differences between $p C T$ and

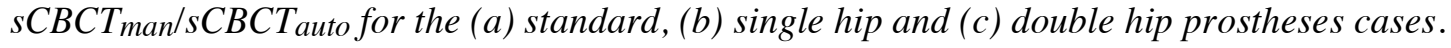




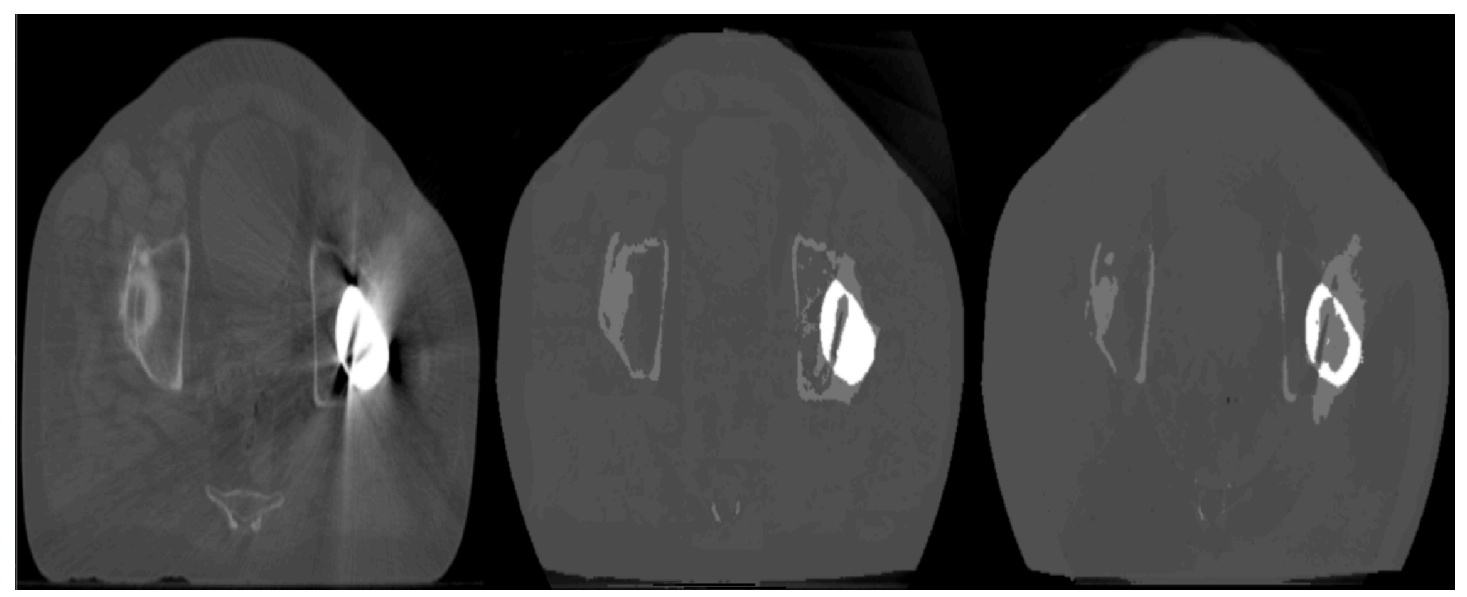

Figure 10.7: A slice of the $p C T$ of the bilateral hip prostheses case (a) and the resultant images after segmentation of CBCT using the manual MLT ( $\left.S C B C T_{\text {man }}\right)$ and the automated $M L T$ ( $s C B C T_{\text {auto }}$ ) ( $b$ and $c$ respectively), showing the $H U$ value difference in the left hip prosthesis.

overestimated the RT hip where scatter and bright streak artefacts were erroneously replaced with hip HU values, leading to a significant reduction in the RT bone volume around that region. Another reason for the underestimation of both bone volumes in both MLT algorithms might be due to the fact that streak artefacts in pCT increased the number of high $\mathrm{HU}$ values and these were not corrected for (only for dose calculation), whereas in SCBCT, both MLT algorithms attempted to correct for this. However, the accuracy of the automated MLT algorithm is comparable to the manual MLT algorithm, except in the LT hip region, and it requires much less (approximately $95 \%$ reduction) operator time.

\subsubsection{Gamma and conformity index comparison}

Table 10.4 shows the $\gamma$ index evaluation for the calculation points falling inside the PTV, rectum and bladder for the $\mathrm{pCT}, \mathrm{sCBCT}_{\operatorname{man}}$ and $\mathrm{sCBCT}_{\text {auto }}$ plans. It shows the fraction of points resulting in $\gamma<1$, for the standard, single hip and double hip prostheses cases. 
Table 10.4: Summary of the $\gamma$ index with fixed DTA $=3 \mathrm{~mm}$ and $D D=3 \%$ for the calculation points falling inside the PTV, rectum and bladder, showing the fraction of points resulting in $\gamma<1$ using $P B, C C$ and $M C$ algorithms.

\section{Standard case}

\begin{tabular}{|c|c|c|c|c|c|c|}
\hline & \multicolumn{3}{|c|}{$\mathbf{s C B C T}_{\text {man }}$} & \multicolumn{3}{|c|}{$\mathbf{s C B C T}_{\text {auto }}$} \\
\hline & PB & $\mathrm{CC}$ & MC & PB & $\mathrm{CC}$ & MC \\
\hline PTV & 100 & 100 & 99.44 & 100 & 100 & 99.5 \\
\hline Rectum & 100 & 99.45 & 97.31 & 100 & 100 & 97.23 \\
\hline Bladder & 100 & 100 & 99.97 & 100 & 100 & 99.98 \\
\hline \multicolumn{7}{|c|}{ Single hip case } \\
\hline PTV & 100 & 99.11 & 98.44 & 100 & 99.98 & 98.99 \\
\hline Rectum & 99.72 & 99.02 & 98.17 & 100 & 100 & 96.75 \\
\hline Bladder & 100 & 98.38 & 98.04 & 100 & 99.52 & 98.46 \\
\hline \multicolumn{7}{|c|}{ Double hip case } \\
\hline PTV & 99.43 & 99.93 & 99.69 & 98.71 & 99.69 & 99.45 \\
\hline Rectum & 99.93 & 99.77 & 98.71 & 99.49 & 99.26 & 97.88 \\
\hline Bladder & 100 & 100 & 99.94 & 100 & 100 & 99.83 \\
\hline
\end{tabular}

For the standard case, almost all calculation points passed the test for the bladder region in both $\mathrm{sCBCT}_{\operatorname{man}}$ and $\mathrm{sCBCT}_{\text {auto }}$ plans, when using all algorithms. In general, going from the $\mathrm{sCBCT}_{\operatorname{man}}$ to $\mathrm{sCBCT}_{\text {auto }}$ plans, the number of calculation points which passed $(\gamma<1)$ increased, except for the rectum region when using the MC algorithm.

For the single hip prosthesis case, the lowest number of points that passed, in the $\mathrm{sCBCT}_{\text {auto }}$ plan, was found in the rectum region when using $\mathrm{MC}$ algorithm, where 96.7\% showed $\gamma<1$ whilst $98.2 \%$ showed $\gamma<1$ in the sCBCT $_{\text {man }}$ plan. Apart from that, in all other regions and when using all the algorithms, the automated MLT algorithm resulted in a larger number of calculation points that passed the test, compared to the manual MLT algorithm. 
For the double hip prostheses case, all the calculation points in the bladder region passed the gamma test when using the $\mathrm{PB}$ and $\mathrm{CC}$ algorithm, whilst using the MC algorithm, 99.9\% and $99.8 \%$ showed $\gamma<1$ for the $\mathrm{sCBCT}_{\text {man }}$ and $\mathrm{sCBCT}_{\text {auto, }}$, respectively. The lowest number of points that passed was found in the rectum region when using MC algorithm, where $98.7 \%$ showed $\gamma<1$ in the $\mathrm{sCBCT}_{\text {man }}$ plan and 97.9\% showed $\gamma<1$ in the sCBCT $_{\text {auto }}$ plan. Similarly, the automated MLT algorithm resulted in a lower number of calculation points that passed the test than the manual MLT algorithm when using all algorithms. For all three cases, the percentage of calculation points that showed $\gamma<1$, in both the $\mathrm{sCBCT}_{\text {man }}$ and $\mathrm{sCBCT}_{\text {auto }}$ plans, is still considered to be clinically acceptable (Son et al., 2015).

Table 10.5 shows the $\mathrm{CI}$ values of the $\mathrm{pCT}$, $\mathrm{sCBCT}_{\text {man }}$ and $\mathrm{sCBCT}_{\text {auto }}$ plans using PB, CC and MC algorithms for the (a) standard, (b) single hip and (c) double hip prostheses cases. For the standard case, it can be clearly seen that the CI values were improved from $0.78,0.31$ and 0.12 , in the $\mathrm{sCBCT}_{\text {man }}$ plans, to $0.89,0.46$ and 0.24 , in the $\mathrm{sCBCT}_{\text {auto }}$ plans, when using $\mathrm{PB}, \mathrm{CC}$ and $\mathrm{MC}$ algorithms, respectively. This shows that the automated MLT algorithm was more accurate than the manual MLT algorithm if the pCT was considered to be the ground truth for dose calculation, and if there were no additional inhomogeneities in the patient anatomy.

For the single hip and double hip prostheses cases, the CI values dropped compared with the pCT plans when going from the $\mathrm{SCBCT}_{\text {man }}$ plans to $\mathrm{SCBCT}_{\text {auto }}$ plans, using all algorithms. This shows that the manual MLT algorithm was more accurate than the automated MLT algorithm when there were additional inhomogeneities in the patient anatomy. However, the operator time needed to segment CBCT images using the manual MLT algorithms was approximately $90 \%$ and $95 \%$ more than the operator time needed to segment CBCT images using the automated MLT algorithms, for the single hip and double hip prostheses cases, respectively. The length of this operator time needed for the manual MLT algorithms could make it difficult to implement this technique in the clinic. 
Table 10.5: Conformity index (CI) comparison between $p C T$, $s C B C T_{\text {man }}$ and $s C B C T_{\text {auto }}$ plans using $P B, C C$ and $M C$ algorithms.

\begin{tabular}{|c|c|c|c|c|}
\hline & & Standard & Single hip & Double hip \\
\hline \multirow{3}{*}{ pCT } & PB & 0.9 & 1 & 0.9 \\
\hline & $\mathrm{CC}$ & 0.6 & 0.99 & 0.56 \\
\hline & MC & 0.58 & 0.97 & 0.32 \\
\hline \multirow{3}{*}{$\mathbf{s C B C T}_{\text {man }}$} & PB & 0.78 & 0.99 & 0.66 \\
\hline & $\mathbf{C C}$ & 0.31 & 0.98 & 0.32 \\
\hline & MC & 0.12 & 0.93 & 0.27 \\
\hline \multirow{3}{*}{ sCBCT $_{\text {auto }}$} & PB & 0.89 & 0.98 & 0.55 \\
\hline & $\mathbf{C C}$ & 0.46 & 0.97 & 0.14 \\
\hline & MC & 0.24 & 0.91 & 0.17 \\
\hline
\end{tabular}

\subsubsection{Dose point comparison}

Figure 10.8 shows the dose difference between the $\mathrm{pCT}$ and the $\mathrm{sCBCT}_{\text {man }} / \mathrm{sCBCT}_{\text {auto }}$ plans at the isocentre using all algorithms for the (a) standard, (b) single hip and (c) double hip prostheses cases, respectively. For the standard case, the largest dose difference between the $\mathrm{sCBCT}_{\text {man }}$ plan and $\mathrm{sCBCT}_{\text {auto }}$ plan, compared with the $\mathrm{pCT}$ plan, was found when using the $\mathrm{CC}$ algorithm, where the difference increased from $0.5 \%$ in the $\mathrm{sCBCT}_{\operatorname{man}}$ plan to $0.9 \%$ in the $\mathrm{sCBCT}_{\text {auto }}$ plan, when compared with the pCT plan (Figure 10.8(a)). Based on the local department condition for re-planning, the $\mathrm{sCBCT}_{\text {man }}$ and $\mathrm{sCBCT}_{\text {auto }}$ plans passed the criteria, showing differences of less than $-2 \%$ compared with the pCT plan when using all algorithms (see Section 1.3).

For the single hip prosthesis case, both the $\mathrm{sCBCT}_{\text {man }}$ and $\mathrm{sCBCT}_{\text {auto }}$ plans showed almost similar dose differences with the pCT plans (Figure 10.8(b)). The sCBCT auto plans did not pass the local department condition for re-planning when using the $\mathrm{CC}$ and $\mathrm{MC}$ algorithms. For the $\mathrm{MC}$ algorithm, both the $\mathrm{sCBCT}_{\operatorname{man}}$ and $\mathrm{sCBCT}_{\text {auto }}$ plans did not pass the criteria. This may represent the true difference between the $\mathrm{pCT}$ and CBCT scans due to the actual anatomical difference. It may also suggest that the $\mathrm{sCBCT}_{\text {man }}$ and $\mathrm{sCBCT}_{\text {auto }}$ scans still need some manual corrections to improve the 

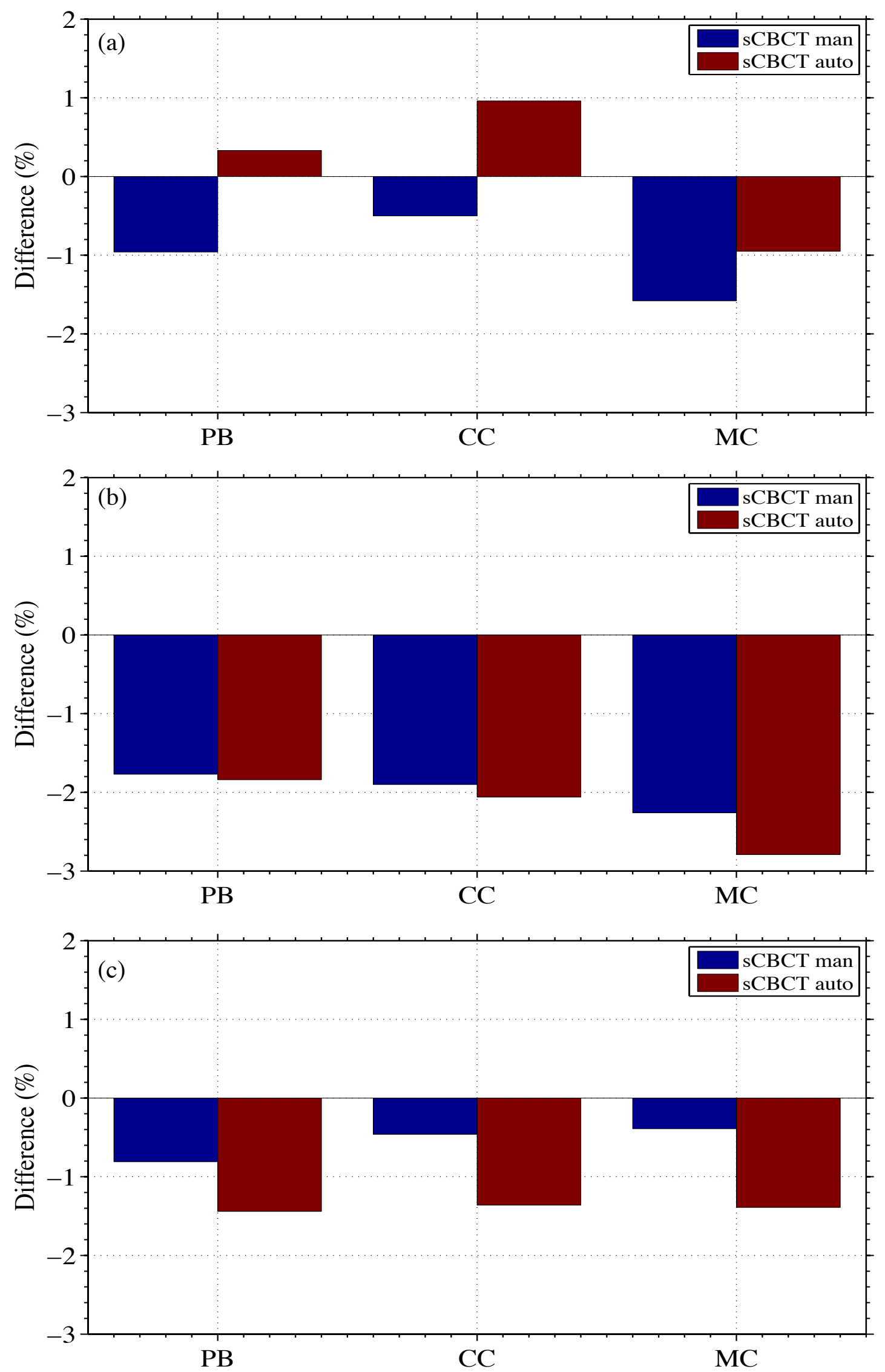

Figure 10.8: Dose comparison between $p C T, s C B C T_{\operatorname{man}}$ and $s C B C T_{\text {auto }}$ plans at the isocentre using $P B, C C$ and $M C$ algorithms for the (a) standard, (b) single hip and (c) double hip prostheses cases. 
accuracy. Even though both the $\mathrm{sCBCT}_{\operatorname{man}}$ and $\mathrm{sCBCT}_{\text {auto }}$ plans showed similar dose differences, the operator time required for defining the threshold values for the different regions in $\mathrm{sCBCT}_{\text {man }}$ was 3.5 hours, whereas in $\mathrm{sCBCT}_{\text {auto, }}$, the threshold values were defined automatically within 20 minutes of operator time.

Figure 10.8(c) shows the dose difference between the pCT and sCBCT plans at the isocentre using all algorithms for the double hip prostheses case. In general, both the $\mathrm{sCBCT}_{\operatorname{man}}$ and $\mathrm{sCBCT}_{\text {auto }}$ plans showed differences of less than $-2 \%$ compared with the pCT plan, when using all algorithms, meaning that both are considered to be clinically acceptable. For the MC calculation, the difference increased from $-0.4 \%$ in the $\mathrm{sCBCT}_{\text {man }}$ plan to $-1.4 \%$ in $\mathrm{sCBCT}_{\text {auto }}$ plan when compared with the pCT plan. However, the operator time required for defining the threshold values for different regions in $\mathrm{sCBCT}_{\operatorname{man}}$ was 8 hours, whilst in $\mathrm{sCBCT}_{\text {auto, }}$, the threshold values were defined automatically and takes 20 minutes operator time. Some manual modification was still needed to ensure an appropriate assignment of each material in the $\mathrm{sCBCT}_{\text {auto }}$ scan to improve the accuracy, but it requires much less (approximately 95\%) operator time compared with the $\mathrm{sCBCT}_{\operatorname{man}} \mathrm{scan}$.

Recently, Dunlop et al (2015) assessed the CBCT dose calculation accuracy for density override approaches for four pelvis cases, where CBCT voxels were assigned as water only and then as either water or bone (water only and water-and-bone methods). This was then compared with a scatter correction and automated density override approach that is available in the RayStation TPS (V3.99, RaySearch Laboratories, Stockholm, Sweden) (Dunlop et al., 2015). The results showed that the automated approach was superior to the other methods, when applied to smaller patients (with anterior-posterior distance $<25 \mathrm{~cm}$ ). For larger patients, the water only method gave the best accuracy. However, in this study, the anterior-posterior distances of the standard, single hip and double hip prostheses cases that were used to test the accuracy of the automated MLT algorithm were $25.6 \mathrm{~cm}, 24 \mathrm{~cm}$ and $26.5 \mathrm{~cm}$, respectively. This shows that the automated MLT algorithm worked sufficiently well with different patient sizes and, as mentioned above, even in the worst scenario i.e. bilateral hip prostheses.

As a result, dividing $\mathrm{CBCT}$ images into five concentric rings was accurate enough to correct image artefacts and the variation in the pixel value with position in the 
CBCT images, even in the complex example of a patient with bilateral hip prostheses. Therefore, the automated MLT algorithm reduced the operator time, but retained an acceptable level of accuracy. This reduction in operator time could turn this researchbased technique into a clinically implemented application and therefore makes it superior compared with the manual approach.

\subsection{Conclusion}

The segmentation of CBCT images using the two MLT methods in this study can be used for dose calculation with an acceptable level of accuracy. Based on previous chapters (Chapter 8 and 9), the results showed that the main disadvantage of the manual MLT algorithm was the length of the operator time needed to segment CBCT images, which could make it difficult to implement it in the clinic. In this chapter, an automated MLT algorithm was developed to reduce the operator time associated with the manual MLT algorithm. The results showed that the automated MLT algorithm showed better dose accuracy than the manual MLT algorithm, compared with the pCT, for a standard (no additional inhomogeneities in the patient anatomy) prostate cancer patient. For a patient with a single hip prosthesis, the automated MLT algorithm, the $\mathrm{sCBCT}_{\text {auto }}$ plans, were comparable to the manual MLT algorithm, the sCBCT $_{\text {man }}$ plans, and showed similar dose differences with the pCT plans, but required less (approximately 90\%) operator time. For a patient with bilateral hip prostheses, the manual MLT algorithm showed smaller dose differences with the pCT than those of the automated MLT algorithm. Nevertheless, those dose differences are still considered to be clinically acceptable. However, some manual modification, to ensure an appropriate assignment of each material in the $\mathrm{sCBCT}_{\text {auto }}$ scan, was still needed to improve the accuracy but it requires much less (approximately 95\%) operator time compared with the $\mathrm{sCBCT}_{\operatorname{man}}$ scan. This reduction in operator time could make it easier to implement in the clinic and makes it superior compared with the manual approach. Therefore, it can be used as a quick-decision-making tool for replanning. Finally, it is envisaged that the method could be applicable for the automation of dose calculation on segmented magnetic resonance (MR) images and could be of interest to MR-based ART. Parts of this chapter (double hip case only) have been published (Almatani et al., 2016b). The next step is to enable dose calculations to be performed with the use of MR images using the MLT algorithm. 
Chapter 11 MR-based dose calculation of a prostate cancer patient 


\subsection{Introduction}

For all the patient cases presented in previous chapters of this thesis, the prostates were outlined using MR imaging which is the gold standard imaging modality for prostate delineation and disease staging (Fütterer et al., 2008, Murphy, 2011). Compared with conventional CT, MR provides much better soft tissue contrast of the prostate, the surrounding normal tissues and OARs. In CT, identifying the prostate boundaries is challenging, whilst in MR images the boundaries of the prostate as well as the peripheral zone and central gland can be identified (Schmidt and Payne, 2015). In addition, MR imaging can provide more clinical information, such as physiological and biochemical information, that cannot be provided by CT images, and can thus distinguish between healthy and malignant tissues (Mayles et al., 2007). Most importantly, MR images are acquired using radiofrequency (non-ionising) radiation, whilst CT images are acquired using ionising radiation. Therefore, it is more advantageous and beneficial to use MR imaging in cases where additional radiation doses need to be avoided or in cases where frequent image acquisitions are required during the treatment course to detect any changes in the target and OARs and to show any early response to therapy or radiation-induced tissue changes (Schmidt and Payne, 2015). These advantages of MR imaging have led to the development of MR imaging systems to be extended for the entire radiotherapy process (Lagendijk et al., 2014a). Recently, a MRI-only simulator has been commercially introduced with features similar to a CT simulator, such as a flat table, external lasers and MRcompatible patient immobilization (Weber et al., 2008, Devic, 2012, Doemer et al., 2015). Owing to the high soft tissue contrast, the GTVs defined in a MR simulator can be significantly smaller than those delineated on a CT (Weber et al., 2008). Such a difference in the target delineation could make the MRI-only simulator a routine modality as it reduces radiation toxicity and eliminates the MR-to-CT registration systematic errors (Korhonen, 2015, Edmund et al., 2015).

With the development of more advanced radiotherapy treatment planning, such as IMRT and stereotactic body radiotherapy (SBRT), the desire for more accurate localization of tumours prior to and during the treatment delivery has increased, ensuring that the higher tumour dose is achieved whilst reducing the dose to OARs and normal tissues. The integration of the CBCT imaging system in the Linac provides $3 \mathrm{D}$ volumetric images during the treatment delivery. However, despite its 
major improvement in IGRT, the image quality of CBCT images makes it hard to accurately identify the prostate and, more importantly, the CBCT imaging dose limits the frequency with which this technique can be used (see Chapter 2). For these reasons, the MR imaging system has been integrated in the Linac system for offline/online treatment guidance, such as MRI-Linac (Lagendijk et al., 2014b). The MR imaging system has also been integrated in the Cobalt source unit as ViewRay, and installed in the treatment room as the MR-on-rails (Mutic and Dempsey, 2014, Stanescu et al., 2014). Therefore, using a MR imaging system as an IGRT tool allows the acquisition of 3D volumetric images immediately before each fraction, without any additional ionizing radiation dose to the patient (radiofrequency radiation). For a highly mobile and challenging target, such as a prostate, MR imaging allows more accurate localization of the prostate and intraprostatic lesions, as well as real-time imaging during beam delivery and thus provides information for ART. Therefore, MR imaging can potentially manage interfraction and intrafraction motions. This would potentially decrease the CTV-PTV margin and increase the confidence of boosting the target dose using fewer treatment fractions (hypofractionation). However, a technical problem of integrating a MR imaging system in a Linac is the magnetic interaction between the two systems (Lagendijk et al., 2016). This would affect the dose distribution inside the patient, leading to a decreased build-up distance, off-axis shift, and asymmetric penumbral (Raaymakers et al., 2004, Oborn et al., 2010, Bol et al., 2012, Rubinstein et al., 2014). In addition, the patient skin dose is increased due to the electron return effect (ERE), where the secondary electrons released inside air regions within the patient are forced back into the tissue, this is most prominent at tissue-air interfaces (Raaijmakers et al., 2005, Murphy, 2011). These effects should be included in the TPS dose calculation algorithms to provide accurate dose calculations. The commercial dose calculation algorithms presented in this thesis, $\mathrm{PB}, \mathrm{CC}$ and $\mathrm{MC}$ EGSnrc, do not include these effects (Bol et al., 2012). The MC algorithm, such as GEANT4 or FLUKA, is suitable for accurate dose calculations in the presence of a high magnetic field (Raaijmakers et al., 2007).

Despite its excellent soft tissue contrast, there are factors that can limit the implementation of some MR imaging platforms into the radiotherapy process. These factors include cost, system-related and patient-related geometric distortions, and ED information for dose calculation (Schmidt and Payne, 2015). In MR images, assigning 
signal values in their spatial location is based on varying the static magnetic field with magnetic field gradients to provide a linear relationship between resonant frequency and position (Korhonen, 2015, Schmidt and Payne, 2015). Therefore, any change in the static magnetic field uniformity and linearity, as well as the patient-specific spatial distribution of magnetic susceptibilities, can cause geometric distortions (Kapanen et al., 2013, Korhonen, 2015). However, one of the main factors that limits the use of MRI-only treatment planning, and the MRI-only simulator, is that MR images do not provide HUs and the intensity, or voxel values are not directly related to EDs (Schmidt and Payne, 2015).

There are different approaches to convert the voxel values of MR images into HUs to produce pseudo-CT images, also known as substitute CT or synthetic CT (Johansson et al., 2011, Hsu et al., 2013, Korhonen et al., 2014, Andreasen et al., 2016, Koivula et al., 2016). One of these approaches uses a CT-based ED atlas with non-rigid registration to transfer CT ED to MR images (Dowling et al., 2012, Andreasen et al., 2016). For prostate cancer patients using this approach, the dose difference between the pseudo-CT and planning CT is within 2\%. A limitation of this approach is that it can be time consuming for multiple atlas and lead to greater uncertainty if the patient is dissimilar to the database used for the atlas (Keereman et al., 2010, Andreasen et al., 2016).

Another approach for converting voxel values in MR images into HUs is by segmenting MR images into a number of materials and subsequently applying bulk density assignment (Eilertsen et al., 2008, Lambert et al., 2011, Karotki et al., 2011, Johansson et al., 2011, Doemer et al., 2015, Koivula et al., 2016, Andreasen et al., 2016). In this approach, the whole body can be considered as water-only, or water and air materials (Schmidt and Payne, 2015). Lambert et al (2011) showed that the bone region should be included for a more accurate dose calculation in the pelvic region. This is in agreement with our findings for accurate dose calculation using sCBCT images (see Chapter 6 and 7). However, bone regions and boundaries are hard to visualize in conventional or standard MR images (T1- or T2-weighted), which is the standard sequence for diagnosis, due to the low proton densities in these regions and short T2 (transverse relaxation time) (Keereman et al., 2012, Gutierrez et al., 2015). Therefore, it is hard to differentiate between air, lung and bone regions. To separate 
bone voxels, additional MR sequences are used, such as ultra-short echo times (UTE) or $\mathrm{T} 1 / \mathrm{T} 2 *$-weighted, where $\mathrm{T} 1$ is the longitudinal relaxation time (Johansson et al., 2011, Kapanen and Tenhunen, 2013, Korhonen et al., 2014). However, these MR sequences may increase SNR at tissue interfaces, and consequently lead to greater uncertainty in the conversion to HUs (Rank et al., 2013, Andreasen et al., 2016). In addition, these extra MR sequences are time consuming and are not used in clinical radiotherapy. The bone regions can be manually or automatically contoured and then assigned with different HUs, whilst soft tissues are converted to HUs using dual model conversion techniques (Korhonen et al., 2014, Koivula et al., 2016). However, for a standard MR sequence, bone regions can be manually or automatically contoured and then assigned with a single HU, and anything outside is considered to be water and is assigned a single HU. This approach, excluding the water-only method, resulted in a dose accuracy of 1-3\%. In this chapter, this method was used to convert the voxel values of the MR images, of the standard prostate case used in Chapter 7, into HUs using the MLT algorithm. These processed images were then used for dose calculations and compared with the original pCT plan.

\subsection{Methodology}

\subsubsection{Modification of MR image}

The acquisition of the MR images of the standard prostate patient was performed with the T2-weighted sequence using the Toshiba scanner (3 Tesla, Model 200 SP5), with a flat table as in the CT simulator. This acquisition sequence is the standard sequence used in the local department for prostate localization and delineation. The matrix size of the reconstructed MR images was $512 \times 512$ and the voxel sizes were $0.74 \times 0.74 \times$ $5 \mathrm{~mm}^{3}$ whilst the CT voxel sizes were $1.19 \times 1.19 \times 3 \mathrm{~mm}^{3}$. As mentioned before, acquiring MR images with the conventional or standard sequence produces images with no contrast between bone and air (Figure 11.1b). In addition, the femoral heads are mostly composed of bone marrow tissue, which has a wide intensity range due to the proportions of adipose and water (Korhonen et al., 2014). Therefore, it is challenging to accurately contour bone regions. However, MATLAB scripts were developed so that contours could be drawn on the MR images. There were 5 contours drawn on the MR image to enable accurate bone representation, and to isolate bone 


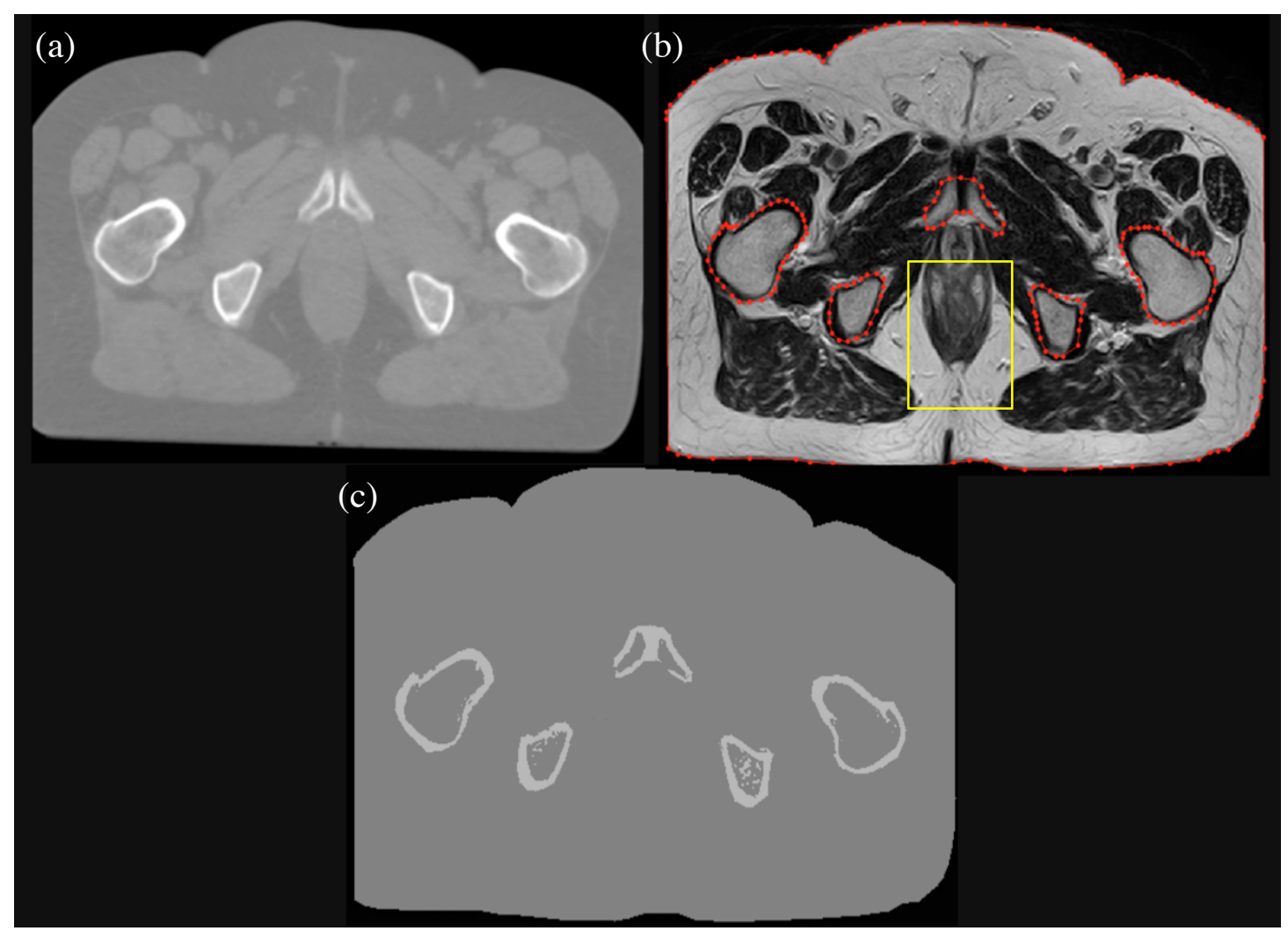

Figure 11.1: A prostate patient scan using (a) $p C T$, (b) $M R$ and the resultant image after segmenting $M R$, (c) $s M R$.

tissue from the surrounding adipose and water tissues, as shown in Figure 11.1b. This was done for each MR slice (total of 24 slices), and the operator time was about 2 hours. In each ROI, the MLT algorithm was applied to convert voxel values into CT numbers that are comparable with the pCT to generate segmented MR (sMR) images (Figure 11.1c). Furthermore, an additional contour was drawn as a body contour around the patient. To reduce manual delineation or contouring time, regional coordinates were written in the MLT algorithm that were expected to encompass the whole rectum, through all the slices. Any voxel inside this region with a range voxel value between 0 and 110 was considered as air with $-976 \mathrm{HU}$ otherwise they were considered as water with $0 \mathrm{HU}$. Any voxel inside the body contour and outside both the bone contours and the rectum region was considered as water. Any voxel inside the bone contours with a range value between 0 and 1150 was considered as bone with $528 \mathrm{HU}$ otherwise they were considered as water. Moreover, as shown in Figure 11.1, there is a missing volume laterally in the MR images, so the external contour of the MR images was copied onto the pCT and any voxel outside this contour was considered as air, thus matching the FOV between the two scans. Finally, to enable 
dose calculation to be performed within sMR images using OMP, the DICOM tags were modified to match pCT DICOM tags, otherwise OMP calculates the dose with overriding the density.

\subsubsection{Treatment planning evaluation and comparison}

The fusion of the sMR and pCT images was accomplished with manual rigid registration. The pCT images were resampled to the sMR images to enable direct comparison of dose calculations. The resultant pCT data set contained 24 slices with a voxel size of $0.74 \times 0.74 \times 5 \mathrm{~mm}^{3}$. The structure sets were then transferred to the sMR images without any modification except the external contour. The plans were then copied to sMR using the same geometry and MU values and the doses were recalculated using $\mathrm{PB}, \mathrm{CC}$ and $\mathrm{MC}$ algorithms (see Chapter 7). The DVHs were generated for PTV, rectum, bladder and left and right femoral heads structures, and compared between pCT and sMR plans using CERR. The gamma index analysis was performed using the pCT plan as a reference, in the PTV, rectum, bladder and left and right femoral heads structures. The same criteria used in the previous chapters were used. Finally, dose at the isocentre (at the geometric centre of the prostate PTV) was compared between the pCT and sMR plans.

\subsection{Results and discussion}

\subsubsection{DVH comparison}

Figure 11.2 shows the DVH of the prostate IMRT plan with a prescription dose of 60 Gy in 20 fractions. It shows the dose of the pCT and sMR plans to the PTV, rectum, bladder, right and left femoral head using the PB (a), CC (b) and MC (c) algorithms.

It can be seen that the sMR plan is in a good agreement with the pCT plan. In general, the sMR plan slightly underestimated the dose to all the structures when using all algorithms. There are some differences and outlines but the general trend holds. The largest differences were found in the rectum minimum dose where it was underestimated by $95.65 \%$ when using PB and CC algorithms, as shown in Table 11.1. This is because the rectum minimum dose was low at $2.3 \mathrm{~Gy}, 2.3 \mathrm{~Gy}$ and $1.9 \mathrm{~Gy}$ when using $\mathrm{PB}, \mathrm{CC}$ and $\mathrm{MC}$ algorithms, respectively, in the pCT plan whilst in the 

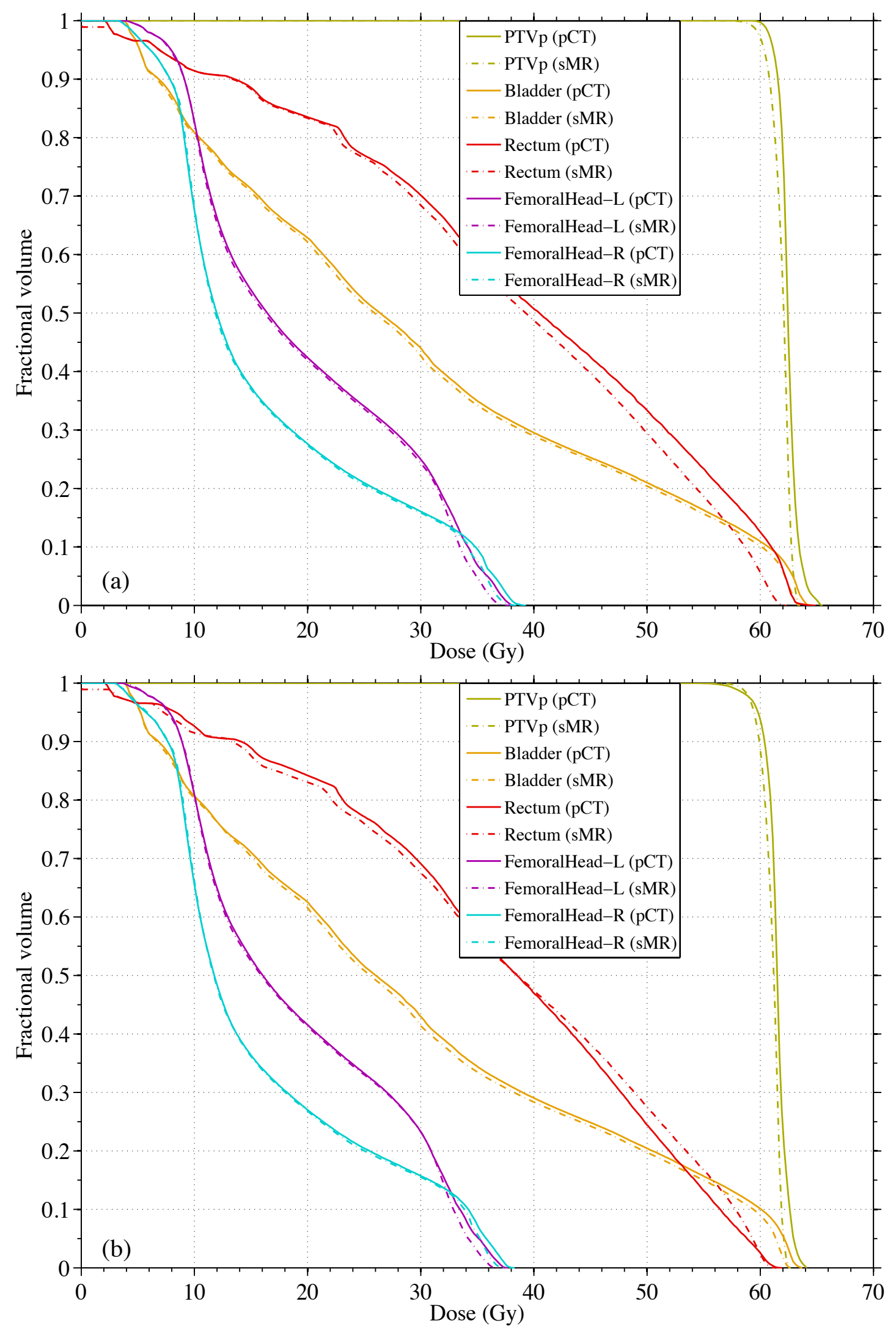


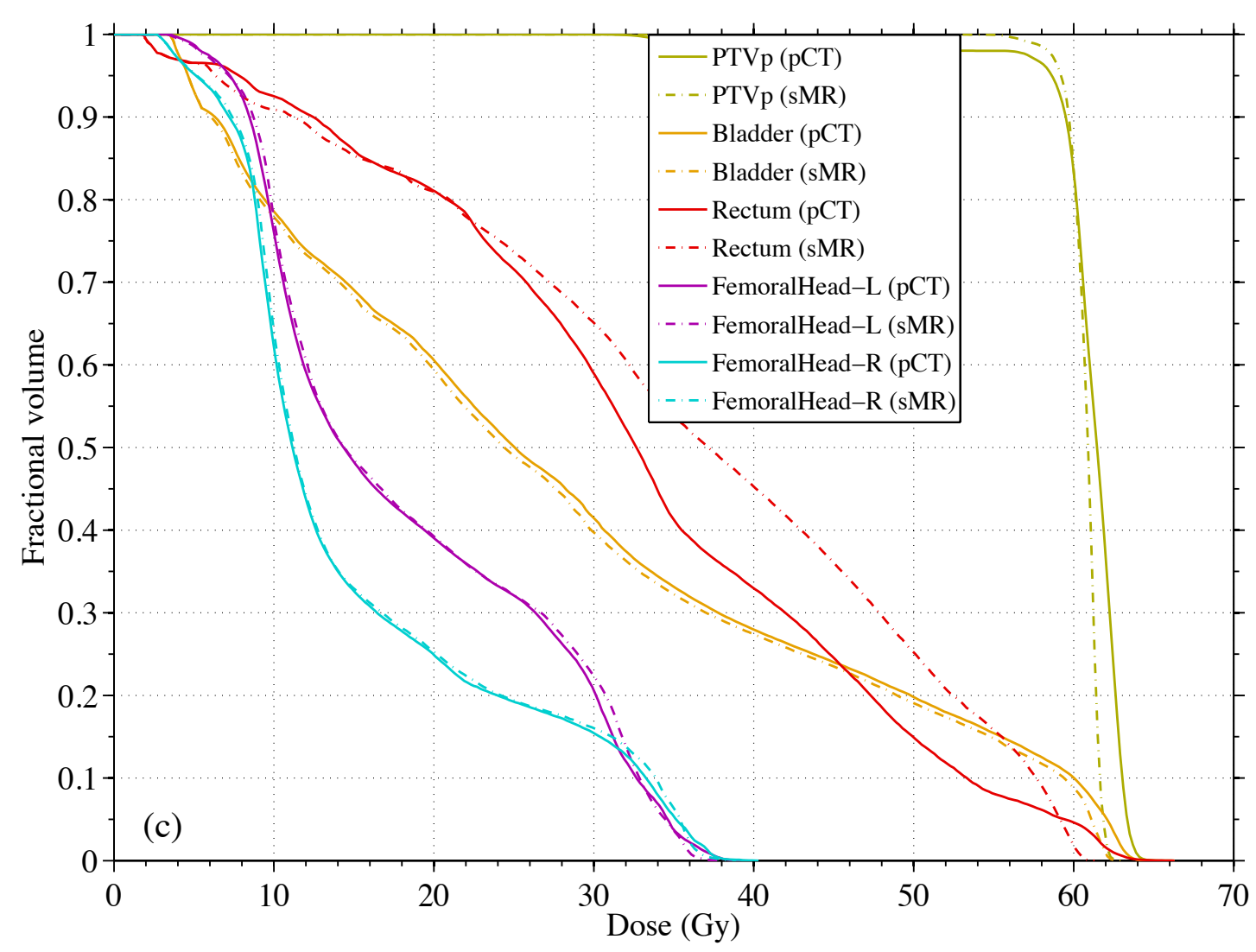

Figure 11.2: DVHs comparison between $p C T$ and sMR plans for PTV, rectum and bladder using (a) $P B$, (b) CC and (c) MC algorithm (prescription dose $60 \mathrm{~Gy}$ ).

sMR plan, the rectum minimum dose was $0.1 \mathrm{~Gy}, 0.1 \mathrm{~Gy}$ and $1.7 \mathrm{~Gy}$ when using PB, $\mathrm{CC}$ and MC algorithms, respectively. Thus a small change in this value leads to a large relative difference. For the PTV, the largest difference was found in the PTV minimum dose where it was overestimated by $70.3 \%$ (21.6 Gy) in the sMR plan when using MC algorithm (Table 11.1). This may be due to the fact that the air/gas pocket volume in the rectum in the sMR was less than in the pCT. Consequently the anterior rectal wall was partially inside the PTV and the MC algorithm was sensitive to such a heterogeneous region, thus, giving lower doses in that region of the $\mathrm{pCT}$ (see section 7.3.1). This rectal volume difference between sMR and pCT scans may be due to a real difference in the rectum volume between the two scans. Another reason for the rectal volume difference may be due to the threshold method, where the partial volume in the rectum was considered either air or water based on the threshold values. For the left and right femoral heads, the largest difference was found for the left femoral head minimum dose where it was underestimated by $-5.4 \%$ and overestimated by $5.4 \%$ when using $\mathrm{CC}$ and $\mathrm{MC}$ algorithms, respectively. For the PB 
algorithm, the left femoral head minimum dose was the same in both plans. However, these findings show that the MLT algorithm used in this study to segment MR images resulted in a dose calculation that is comparable to the $\mathrm{pCT}$.

Table 11.1: Dose and coverage differences between pCT and sMR plans, in \%, for the PTV, rectum, bladder, left and right femoral head.

\begin{tabular}{|c|c|c|c|c|}
\hline & & \multicolumn{3}{|c|}{ SMR } \\
\hline & & PB & $\mathrm{CC}$ & MC \\
\hline \multirow{3}{*}{ PTV } & $\mathbf{D}_{\max }$ & -3.05 & -2.18 & -4.82 \\
\hline & $\mathbf{D}_{\text {mean }}$ & -0.91 & -0.69 & -0.27 \\
\hline & $\mathbf{D}_{\min }$ & -3.70 & 4.50 & 71.28 \\
\hline \multirow{3}{*}{ Rectum } & $\mathbf{D}_{\text {max }}$ & -4.00 & -0.96 & -7.54 \\
\hline & $\mathbf{D}_{\text {mean }}$ & -3.07 & 0.29 & 8.44 \\
\hline & $\mathbf{D}_{\min }$ & -95.65 & -95.65 & -10.52 \\
\hline \multirow{3}{*}{ Bladder } & $\mathbf{D}_{\max }$ & -1.85 & -1.56 & -3.08 \\
\hline & $\mathbf{D}_{\text {mean }}$ & -1.40 & -1.62 & -1.91 \\
\hline & $\mathbf{D}_{\min }$ & 0 & 0 & 0 \\
\hline \multirow{3}{*}{ Left femoral head } & $\mathbf{D}_{\text {max }}$ & -0.52 & -1.06 & -3.05 \\
\hline & $\mathbf{D}_{\text {mean }}$ & -1.31 & -0.87 & 0.86 \\
\hline & $\mathbf{D}_{\min }$ & 0 & 5.40 & -5.40 \\
\hline \multirow{3}{*}{ Right femoral head } & $\mathbf{D}_{\max }$ & -2.03 & -1.56 & -3.47 \\
\hline & $\mathbf{D}_{\text {mean }}$ & -0.84 & -0.61 & 1.22 \\
\hline & $\mathbf{D}_{\text {min }}$ & 0 & 0 & 0 \\
\hline
\end{tabular}

\subsubsection{Gamma index comparison}

Table 11.2 shows the $\gamma$ index evaluation for the calculation points falling inside the PTV, rectum, bladder and right and left femoral heads for the sMR plan using PB, CC and MC algorithms. The results are shown as the percentage of calculation points resulting in $\gamma \leq 1$. As mentioned in section 11.3.1, there was a difference in the rectal volume between the pCT and sMR. This difference resulted in $84 \%, 82 \%$ and $81 \%$ of the calculation points passed the test, for the rectum region, when using $\mathrm{PB}, \mathrm{CC}$ and MC algorithms, respectively. For the left and right femoral head regions, almost all calculation points passed the $3 \% / 3 \mathrm{~mm}$ criteria when using $\mathrm{PB}, \mathrm{CC}$ and $\mathrm{MC}$ 
algorithms. This shows that the MLT algorithm correctly replaced the voxel values with bone $\mathrm{CT} / \mathrm{HU}$ values, with an acceptable level of accuracy.

Table 11.2: Summary of the $\gamma$ index with fixed DTA $=3 \mathrm{~mm}$ and $D D=3 \%$ for the calculation points falling inside the PTV, rectum, bladder, right and left femoral head showing the percentage of points resulting with $\gamma \leq 1$.

\begin{tabular}{cccc} 
& \multicolumn{3}{c}{ sMR } \\
\cline { 2 - 4 } & PB & CC & MC \\
\hline PTV & 94.90 & 97.83 & 99.66 \\
\hline Rectum & 84.05 & 81.92 & 81.44 \\
\hline Bladder & 100 & 100 & 100 \\
\hline Left femoral head & 100 & 100 & 99.87 \\
\hline Right femoral head & 100 & 100 & 99.94 \\
\hline
\end{tabular}

\subsubsection{Dose point comparison}

Table 11.3 shows the dose, in Gy, of the pCT and sMR plans at the isocentre (the geometric centre of the prostate PTV) using PB, CC and MC algorithms. The segmentation of MR images using the MLT algorithm used in this study resulted in a dose difference of $-0.3 \%, 0.8 \%$ and $-1.3 \%$ when using $\mathrm{PB}, \mathrm{CC}$ and $\mathrm{MC}$ algorithms, respectively. Based on the local department condition for re-planning (see Section 8.2.5.3), sMR plan passed the criteria, showing differences of less than $-2 \%$ compared with the pCT plan when using all algorithms.

Table 11.3:Dose comparison between pCT and sMR plans at the isocentre using PB, CC and MC algorithms.

\begin{tabular}{c|ccc|ccc}
\multirow{2}{*}{ Scan } & \multicolumn{3}{|c|}{ pCT } & \multicolumn{3}{c}{ SMR } \\
\cline { 2 - 7 } & PB & CC & MC & PB & CC & MC \\
\hline $\begin{array}{c}\text { Dose at } \\
\text { isocentre (Gy) }\end{array}$ & 61.9 & 60.6 & 61.3 & 61.7 & 61.1 & 60.5 \\
\hline
\end{tabular}




\subsection{Conclusion}

The segmentation and conversion of MR images into HUs/EDs data using the MLT algorithm used in this study can be used for dose calculation. In this chapter, the segmentation of MR images of a prostate cancer patient was investigated as a feasibility study. The MR images were segmented into three material bins, mainly air, water and bone. The bone regions were contoured to isolate bone tissue from the surrounding tissues. Similar dose distribution to the original treatment plan were found when dose calculations were performed on the MLT corrected CBCT images. Gamma evaluation showed that more than more than $94.9 \%$ of the calculation points passed the test, except for the rectum region which may be due to the actual anatomical difference between the $\mathrm{pCT}$ and MR scans. The sMR provides accurate dose calculations with differences of less than $2 \%$. Therefore, this method can be applicable for the dose calculation on MR images and can be of interest to MRI-only based radiotherapy treatment planning and MR-based ART. For MRI-Linac, this method can be performed on MR images taken during the radiotherapy to evaluate the necessity of re-planning. Parts of this chapter have been published (Almatani et al., 2017). 
Chapter 12 Conclusion and future work 


\subsection{Summary and conclusion}

In this thesis, it has been shown that CBCT imaging plays an important rule in the radiotherapy process. The development of CBCT volumetric imaging in the treatment room makes it a widely-used IGRT tool and a routine procedure for patient verification and tumour positioning. Compared with other IGRT techniques, the CBCT system provides 3D image sets with good soft tissue contrast. For prostate cancer patients, the use of CBCT as an IGRT tool to localize the target, ensure dose coverage and evaluate OAR geometry throughout the treatment course has improved the accuracy of the radiotherapy treatment especially for highly conformal treatment techniques. In addition, CBCT can be used as an ART tool to refine and adapt the treatment plan. Ideally, CBCT can be used for dose calculation and to provide information about the treatment dose to the tumour and OARs on a daily or weekly basis for ART purposes.

CBCT images in the XVI system, however, provide pixel values, gray-scale intensity values or CBCT numbers that do not demonstrate true HUs as CBCT images contain more scatter than conventional CT. Consequently, CBCT images cannot be used directly for dose calculation. If there are significant anatomical changes observed on the CBCT images, acquiring another $\mathrm{CT}$ is necessary for an accurate assessment of dose differences. This procedure is time consuming across, all staff groups involved in the radiotherapy pathway, and has an element of risk associated with it. Thus it would be efficient to use CBCT images that were already taken during radiotherapy for evaluating the necessity of re-planning.

The main aim of this thesis was to enable fast and accurate dose calculation to be performed on CBCT images. In order to correct CBCT data, the MLT was used to categorise pixel values in the CBCT images into segments of homogeneous HU. The MLT method was performed on CBCT images of a phantom, standard prostate patient, prostate cancer patients with a single and double metallic prosthetic hip replacements. This enabled the development of a robust method to account for the full range of patient sizes as well as the difficulties presented by the metal artefacts in both pCT and CBCT images. In addition, MC modeling was used in order to separate uncertainties in the dose calculation inherent in the treatment planning system, including those due to the influence of the titanium implant, from uncertainties 
introduced by the threshold method.

In Chapter 5, the Elekta Synergy 6MV linear accelerator was modelled using EGSnrc code (BEAMnrc/DOSXYZnrc). The fine-tuned parameters for the $6 \mathrm{MV}$ photon beam was found to be a $6.05 \mathrm{MeV}$ mean electron energy with a $0.14 \mathrm{~cm}$ circular spot size. The validation process of the MC Linac model involved the comparison of PDD, dose profile, QI and OF, for open and wedged beams, with the measurement. The MC model was in agreement with the measurements for fields ranging from $5 \times 5$ to $20 \times 20 \mathrm{~cm}^{2}$. The validated MC Linac model was used to generate clinical MC-based treatment plans for the following studies.

In Chapter 6, the phantom case, it was demonstrated that if the CBCT images are used directly for dose calculation, even for a very simple phantom, it results in a dose difference of more than $10 \%$ when compared with pCT images. In addition, it resulted in more than $70 \%$ of the calculation points failing the $\gamma$ test. The segmentation of CBCT into two materials (water and bone) and converting the CBCT numbers to CT numbers, that are comparable to the $\mathrm{pCT}$, using the MLT algorithm results in a dose difference of $2 \%$.

In Chapter 7, the standard prostate patient case, it was demonstrated that the segmentation of CBCT images into three materials (air, water and bone) using the MLT algorithm, provides accurate dose calculations with differences of less than $2 \%$. The CBCT image artefacts, such as streak, cupping, ring and radar artefacts, were corrected and accurately replaced with the correct CT numbers.

In Chapter 8, the prostate patient with a single hip prosthesis case, the amount of scatter and image artefacts was increased due to the presence of the metal. As a result, it was necessary to extend the MLT algorithm to categorise pixel values into segments on a region-by-region basis, with the region size changing depending on the anatomical features. In general, the greater the variation in the scatter the greater the number of regions that need to be considered and the size of the region decreases as it gets closer to inhomogeneities, e.g. metal prosthetic implant. In addition, a larger number of materials (up to 8 ) than typically used in previous works was explored. The result demonstrated that the dose difference between pCT and SCBCT plans decreased as the number of materials or bins increased as well as the operator time. The optimal 
balance of dose accuracy $(-1.9 \%)$ and operator time (3.5 hours) was found when five values of HU (air, adipose, water, bone and metal implant HU values) were used.

In Chapter 9, the prostate with double hip prostheses case, the CBCT images were segmented into five values of $\mathrm{HU}$ in three different sets, where the only difference was regarding the bone volume considered in the segmentation process. The operator time for segmenting the $1^{\text {st }} \mathrm{sCBCT}, 2^{\text {nd }} \mathrm{sCBCT}$ and $3^{\text {rd }} \mathrm{sCBCT}$ was 8,12 and 14 hours, respectively. The result showed that the $1^{\text {st }}$ SCBCT scan, even though it showed the largest difference in the bone volumes compared with the pCT scan, gave the best balance between the dose accuracy $(-0.8 \%)$ and operator time ( 8 hours).

The length of operator time needed to segment CBCT images, for single and double hip prostheses cases, could make it difficult to implement this as a technique in the clinic. In Chapter 10, an automated MLT algorithm was developed to reduce the operator time associated with the manual MLT algorithm. The automated MLT algorithm was used to segment CBCT images of the previous patient cases. The results showed that automated MLT algorithm were comparable to the manual MLT algorithm and showed similar dose differences to the pCT plans, but required less (approximately $90 \%$ and $95 \%$ for single and double hip prostheses case, respectively) operator time. This reduction in operator time would make it easier to implement in the clinic and makes it superior compared with the manual approach as the main reason for proposing this method is to use it as a quick-decision-making tool for replanning.

Finally, in Chapter 11, the MR images of the standard prostate cancer patient, used previously, was used for dose calculation. The MR images were segmented into three material bins (air, water and bone) using the MLT algorithm. The bone regions were contoured to isolate bone tissue from the surrounding tissues and the operator time was approximately 2 hours. In these regions, specific threshold values were applied to convert the voxel values into $\mathrm{HU}$ of either bone or water. Any voxel outside these bone regions were considered either water or air. The results showed that the segmentation of MR images using the method used in this study provides accurate dose calculations with differences of less than $2 \%$. This method can be of interest to MRI-only based radiotherapy treatment planning and MR-based ART. 


\subsection{Future work}

The results of this thesis allow implementing ART techniques and underline the need for further investigations, as following :

1. Implementing the automated MLT algorithm for hypofractionated high dose IMRT for prostate, where 500 or 600 cGy per fraction, the PACE trial (Cancer Research UK PACE trial, 2016). In this case, 200 cGy could be delivered to the patient and then acquiring and segmenting $\mathrm{CBCT}$ images then rapidly replanning and optimizing that treatment plan. This can be done two or three times with one treatment slot.

2. Finding the optimum number of bins that are needed when segmenting CBCT images for different sites such as lung or head and neck cases, even in the complex example of a patient with dental fillings and implants.

3. Investigating the dose calculation accuracy based on segmented MR images using MLT algorithm for prostate patients with hip prostheses and for different sites.

4. Developing an automated algorithm for contouring bone regions in MR images, as well as an automated MLT algorithm, to reduce the operator time.

\subsection{Thesis conclusion}

This thesis reached its objectives in that it:

1. Enabled dose calculations to be performed with the use of CBCT images using MLT algorithm.

2. Developed a robust method to account for the full range of patient size as well as the difficulties presented by the metal artefacts in both pCT and CBCT images.

3. Found the optimum number of bins that are needed when segmenting CBCT images to give the best balance between dose accuracy and operator time (Almatani et al., 2016a).

4. Identified the effects of these bins on the dose calculation algorithms when using simple and complicated plan geometries. 
5. Developed an automated MLT algorithm and enhanced the performance of this method so it can be completed in a timeframe which can make it clinically useful. Then it was used as a fast decision-making tool regarding on-treatment patient shape changes and whether a new CT is required (Almatani et al., 2016b).

6. Enabled dose calculations to be performed with the use of MR images using the MLT algorithm.

The endpoint of this thesis was achieved which was to have an accurate and efficient way to modify CBCT data where it can be quickly used for dose calculation that is as accurate as using the TPS to calculate a dose distribution on a CT planning scan, then using it as a quick-decision-making tool for re-planning, as shown in Figure 1.6. 


\section{Appendices}

\section{Appendix A BEAMnrc input example}

Beam_6MV_Elekta \#!GUI1.0 AIR700ICRU

$0,0,0,0,1,3,1$, IWATCH ETC.

$1000,851,266,50000.0,2,1000,2,0$, NCASE ETC.

$15,100,0,0,0$, , DIRECTIONAL BREM OPTIONS

$-1,19,-0.14,0.0,0.0,1,0,-0.0,0.0,0.0$, IQIN, ISOURCE + OPTIONS

0, MONOENERGETIC

6.05

$0,0,0.7,0.01,0,0,0,0$, ECUT,PCUT,IREJCT,ESAVE

$0,1,1,7,7$, PHOTON FORCING

1,11 , SCORING INPUT

5,1

5.0, 10.0, 15.0, 20.0, 25.0,

0 , DOSE COMPONENTS

$0.0, \mathrm{Z}$ TO FRONT FACE

$* * * * * * * * * * *$ start of CM SLABS with identifier target $* * * * * * * * * * *$

10.0, RMAX

WRe and copper block target

2, NSLABS

0, ZMIN

$0.1,0.7,0.01,0,1,1.0$

WRE_18P0_700

$1,0.7,0.01,0,1,1.0$

CU700ICRU

************ start of CM CONS3R with identifier primcoll $* * * * * * * * * * *$

10.0, RMAX

Primary Collimator

$1.5, \mathrm{ZMIN}$

10.1, ZTHICK

2, NUM_NODE

$1.5,0.645$,

$11.6,2.9$,

$0.7,0.01,0,0,0$,

AIR700ICRU

$0.7,0.01,0,2,0$,

tangsten_Alloy

$* * * * * * * * * * *$ start of CM FLATFILT with identifier flattenf $* * * * * * * * * * *$

10.0, RMAX

Flattening Filter

13.39, ZMIN

6, NUMBER OF LAYERS

1, 0.34, \# CONES, ZTHICK OF LAYER 1

0 ,

0.545 ,

1, 0.27, \# CONES, ZTHICK OF LAYER 2 
0.545 ,

0.9 ,

1, 0.49, \# CONES, ZTHICK OF LAYER 3

0.9 ,

1.45 ,

$1,0.55$, \# CONES, ZTHICK OF LAYER 4

1.45 ,

2.25 ,

3, 0.56, \# CONES, ZTHICK OF LAYER 5

2.25, 3.85, 4.65,

$3.25,3.85,4.65$,

1, 0.2, \# CONES, ZTHICK OF LAYER 6

4.65,

4.65,

$0.7,0.01,0,3$,

MILDSTEEL700

$0.7,0.01,0,0$,

AIR700ICRU

$0.7,0.01,0,0$,

MILDSTEEL700

$0.7,0.01,0,0$,

AIR700ICRU

$0.7,0.01,0,0$,

MILDSTEEL700

0.7, 0.01, 0, 0,

AIR700ICRU

$0.7,0.01,0,0$,

MILDSTEEL700

0.7, 0.01, 0, 0,

AIR700ICRU

$0.7,0.01,0,0$,

MILDSTEEL700

$0.7,0.01,0,0$,

AIR700ICRU

$0.7,0.01,0,0$,

MILDSTEEL700

0.7, 0.01, 0, 0,

AIR700ICRU

$0.7,0.01,0,0$,

MILDSTEEL700

$0.7,0.01,0,0$,

AIR700ICRU

$* * * * * * * * * * *$ start of CM CHAMBER with identifier chamber $* * * * * * * * * * *$

7.4, RMAX

ion chamber

16.44, ZMIN

$2,15,2$, N_TOP, N_CHM, N_BOT

$0.0012,7.4,0$, ZTHICK, RCYS, FLAG FOR LAYER 1 IN TOP

$0.7,0.01,0,0$,

MYLAR700ICRU 
$0.511,0.01,0,0$,

AL700ICRU

0.1, 4.5, 0, ZTHICK, RCYS, FLAG FOR LAYER 2 IN TOP

$0.7,0.01,0,0$,

AIR700ICRU

$0.7,0.01,0,0$,

AL700ICRU

4.5, 5.04, 6.625, RADII FOR CENTRAL PART

$0.0012,0$, ZTHICK, FLAG FOR LAYER 1 IN CENTRAL PART

$0.7,0.01,0,0$,

MYLAR700ICRU

$0.1,0$, ZTHICK, FLAG FOR LAYER 2 IN CENTRAL PART

$0.7,0.01,0,0$,

AIR700ICRU

0.0012, 0, ZTHICK, FLAG FOR LAYER 3 IN CENTRAL PART

$0.7,0.01,0,0$,

MYLAR700ICRU

0.22, 0, ZTHICK, FLAG FOR LAYER 4 IN CENTRAL PART

$0.7,0.01,0,0$,

AIR700ICRU

0.0012, 0, ZTHICK, FLAG FOR LAYER 5 IN CENTRAL PART

$0.7,0.01,0,0$,

MYLAR700ICRU

$0.1,0$, ZTHICK, FLAG FOR LAYER 6 IN CENTRAL PART

$0.7,0.01,0,0$,

AIR700ICRU

0.0012, 0, ZTHICK, FLAG FOR LAYER 7 IN CENTRAL PART

$0.7,0.01,0,0$,

MYLAR700ICRU

0.1, 0, ZTHICK, FLAG FOR LAYER 8 IN CENTRAL PART

$0.7,0.01,0,0$,

AIR700ICRU

0.0012, 0, ZTHICK, FLAG FOR LAYER 9 IN CENTRAL PART

$0.7,0.01,0,0$,

MYLAR700ICRU

0.1, 0, ZTHICK, FLAG FOR LAYER 10 IN CENTRAL PART

$0.7,0.01,0,0$,

AIR700ICRU

0.0012, 0, ZTHICK, FLAG FOR LAYER 11 IN CENTRAL PART

$0.511,0.01,0,0$,

MYLAR700ICRU

0.1, 0, ZTHICK, FLAG FOR LAYER 12 IN CENTRAL PART

$0.7,0.01,0,0$,

AIR700ICRU

0.0012, 0, ZTHICK, FLAG FOR LAYER 13 IN CENTRAL PART

$0.7,0.01,0,0$,

MYLAR700ICRU

0.1, 0, ZTHICK, FLAG FOR LAYER 14 IN CENTRAL PART

$0.7,0.01,0,0$,

AIR700ICRU 
0.0012, 0, ZTHICK, FLAG FOR LAYER 15 IN CENTRAL PART

$0.7,0.01,0,0$,

MYLAR700ICRU

$0.7,0.01,0,0$, chamber wall

AL700ICRU

$0.7,0.01,0,0$, gap

AL700ICRU

$0.7,0.01,0,0$, container

AL700ICRU

0.3004, 6.625, 0, ZTHICK, RCYS, FLAG FOR LAYER 1 IN BOTTOM PART

$0.7,0.01,0,0$,

AIR700ICRU

$0.7,0.01,0,0$,

AL700ICRU

0.07, 4.5, 0, ZTHICK, RCYS, FLAG FOR LAYER 2 IN BOTTOM PART

$0.7,0.01,0,0$,

AIR700ICRU

$0.7,0.01,0,0$,

AL700ICRU

0 , MRNGE

$* * * * * * * * * * *$ start of CM SLABS with identifier backs $* * * * * * * * * * *$

5.45, RMAX

backscatter plate

1 , NSLABS

18.2, ZMIN

$0.3,0.7,0.01,0,0,0$

AL700ICRU

$* * * * * * * * * * *$ start of CM JAWS with identifier Wedge $* * * * * * * * * * *$

5.4, RMAX

Wedge

1, \# PAIRED BARS OR JAWS

$\mathrm{X}$

18.6, 24.4, 5.4, 5.4, 3, -4.086,

$0.7,0.01,0,0$,

$0.7,0.01,0,0$,

AIR700ICRU

************ start of CM MLCE with identifier ymlc ***********

20, RMAX

$\mathrm{x}$-MLCs for Elekta

0 , ORIENT

40, NUM_LEAF

29.8, 37.3, ZMIN, ZMAX

33.9, 33.7, ZSTEPL, ZSTEPR

$0.06, \mathrm{TGW}$

0.164, 0.201, X3, X4

1, 100, SPACE, SSD

0 , LBROT

0, ENDTYPE

15, 33.55, LEAFRADIUS, CIL

$-15.1567,15.0446,1$ 
$-15.1567,15.0446,1$

$-15.1567,15.0446,1$

$-15.1567,15.0446,1$

$-15.1567,15.0446,1$

$-15.1567,15.0446,1$

$-15.1567,15.0446,1$

$-15.1567,15.0446,1$

$-15.1567,15.0446,1$

$-15.1567,15.0446,1$

$-15.1567,15.0446,1$

$-15.1567,15.0446,1$

$-15.1567,15.0446,1$

$-15.1567,15.0446,1$

$-15.1567,15.0446,1$

$-16.1538,16.0343,1$

$-16.3554,16.341,1$

$-16.4624,16.4487,1$

$-16.4651,16.4534,1$

$-16.4651,16.4537,1$

$-16.3645,16.2521,1$

$-16.1632,16.2521,1$

$-16.0625,16.0511,1$

$-15.8612,15.9501,1$

$-15.056,15.1453,1$

$-15.056,15.1453,1$

$-15.056,15.1453,1$

$-15.056,15.1453,1$

$-15.056,15.1453,1$

$-15.056,15.1453,1$

$-15.056,15.1453,1$

$-15.056,15.1453,1$

$-15.056,15.1453,1$

$-15.056,15.1453,1$

$-15.056,15.1453,1$

$-15.056,15.1453,1$

$-15.056,15.1453,1$

$-15.056,15.1453,1$

$-15.056,15.1453,1$

$-15.056,15.1453,1$

$0.7,0.01,0,0$,

AIR700ICRU

$0.7,0.01,0,0$,

tangsten_Alloy

$* * * * * * * * * * *$ start of CM MLCQ with identifier ybj

28.0, RMAX

Backup jaw

0 , IDMLFC

39.6, ZMIN

3.0, ZTHICK

4, 20, \# LEAVES, TOTAL WIDTH 
0, ZFOCUS(1)

6, 41.1, R0LEAF, ZOLEAF

$-7.7948,7.7809,4$

$0.7,0.01,0,0$,

AIR700ICRU

$0.0,0.01,0,11$,

tangsten_Alloy

$* * * * * * * * * * *$ start of CM JAWS with identifier secjaws $* * * * * * * * * * *$

28.0, RMAX

Secondary jaws

1, \# PAIRED BARS OR JAWS

$\mathrm{X}$

$43.1,50.9,1.724,2.036,-2.155,-2.545$,

$0.0,0.0,0,0$,

$0.7,0.01,0,0$,

tangsten_Alloy

$* * * * * * * * * * *$ start of CM SLABS with identifier airslab $* * * * * * * * * * *$

30.0, RMAX

PMMA light field reticle-air

1 , NSLABS

51.9, ZMIN

$0.103,0.7,0.01,0,12,0.5$

MYLAR700ICRU

$* * * * * * * * * * *$ start of CM APPLICAT with identifier applicat $* * * * * * * * * * *$

28, RMAX

10 x 10 Electron Applicator

82, ZBACK

$1,0, \#$ SCRAPERS, SQUARE

$61,0.6,7,2,0,0$

$0.7,0.01,0,0$,

AIR700ICRU

$* * * * * * * * * * * * * * * * * * * * *$ end of all $\mathrm{CMs} * * * * * * * * * * * * * * * * * * * * * * * * * * * * * *$

\#\#\#\#\#\#\#\#\#\#\#\#\#\#\#\#\#\#\#\#\#

:Start MC Transport Parameter:

Global ECUT $=0.7$

Global PCUT $=0.01$

Global SMAX $=1 \mathrm{e} 10$

ESTEPE $=0.25$

$\mathrm{XIMAX}=0.5$

Boundary crossing algorithm $=$ EXACT

Skin depth for $\mathrm{BCA}=0$

Electron-step algorithm= PRESTA-II

Spin effects $=$ On

Brems angular sampling $=$ Simple

Brems cross sections $=\mathrm{BH}$

Bound Compton scattering= Off

Compton cross sections $=$ default

Pair angular sampling $=$ Simple

Pair cross sections $=\mathrm{BH}$ 
Photoelectron angular sampling $=$ Off

Rayleigh scattering $=$ Off

Atomic relaxations $=$ Off

Electron impact ionization $=$ Off

Photon cross sections $=\mathrm{si}$

Photon cross-sections output $=$ Off

:Stop MC Transport Parameter:

\#\#\#\#\#\#\#\#\#\#\#\#\#\#\#\#\#\#\#\#\#

:Start DBS rejection plane:

Use a rejection plane $=$ On

$\mathrm{Z}(\mathrm{cm})$ from zero reference plane $=85$

:Stop DBS rejection plane:

\#\#\#\#\#\#\#\#\#\#\#\#\#\#\#\#\#\#\#\#\#

:Start BCSE:

Use BCSE $=$ Off

Media to enhance $=$

Enhancement constant $=1000$

Enhancement power $=2$

Russian Roulette $=$ on

:Stop BCSE:

\#\#\#\#\#\#\#\#\#\#\#\#\#\#\#\#\#\#\#\#\#

\section{Appendix B DOSXYZnrc input example}

Dose_6MV_Elekta

\#!GUI1.0

0

/scratch/turki.almatani/egsnrc/dosxyznrc/CTh.egsphant

$0.7,0.01,0$

$0,0,0$,

$2,9,0.1,0.54,-0.7,270,270,18,180,0,20$

2, 0, 0, 0, 0, 0, 0, 0

BEAM_LinD_6MV,Bhip1_1,700icru_tangsten

$1000,0,1000,381,1236,100.0,0,0,1,0,0,0,0,100,0$

\#\#\#\#\#\#\#\#\#\#\#\#\#\#\#\#\#\#\#\#\#

:Start MC Transport Parameter:

Global ECUT $=0.7$

Global PCUT $=0.01$

Global SMAX $=1 \mathrm{e} 10$

$\mathrm{ESTEPE}=0.25$

$\mathrm{XIMAX}=0.5$

Boundary crossing algorithm $=$ EXACT

Skin depth for $\mathrm{BCA}=0$

Electron-step algorithm= PRESTA-II 
Spin effects $=$ On

Brems angular sampling $=$ Simple

Brems cross sections $=\mathrm{BH}$

Bound Compton scattering $=$ On

Compton cross sections $=$ default

Pair angular sampling $=$ Simple

Pair cross sections $=\mathrm{BH}$

Photoelectron angular sampling $=\mathrm{On}$

Rayleigh scattering $=$ On

Atomic relaxations $=$ On

Electron impact ionization $=$ On

Photon cross sections $=\mathrm{si}$

Photon cross-sections output $=$ Off

:Stop MC Transport Parameter:

\#\#\#\#\#\#\#\#\#\#\#\#\#\#\#\#\#\#\#\#\#

\section{Appendix C MLT automated algorithm example}

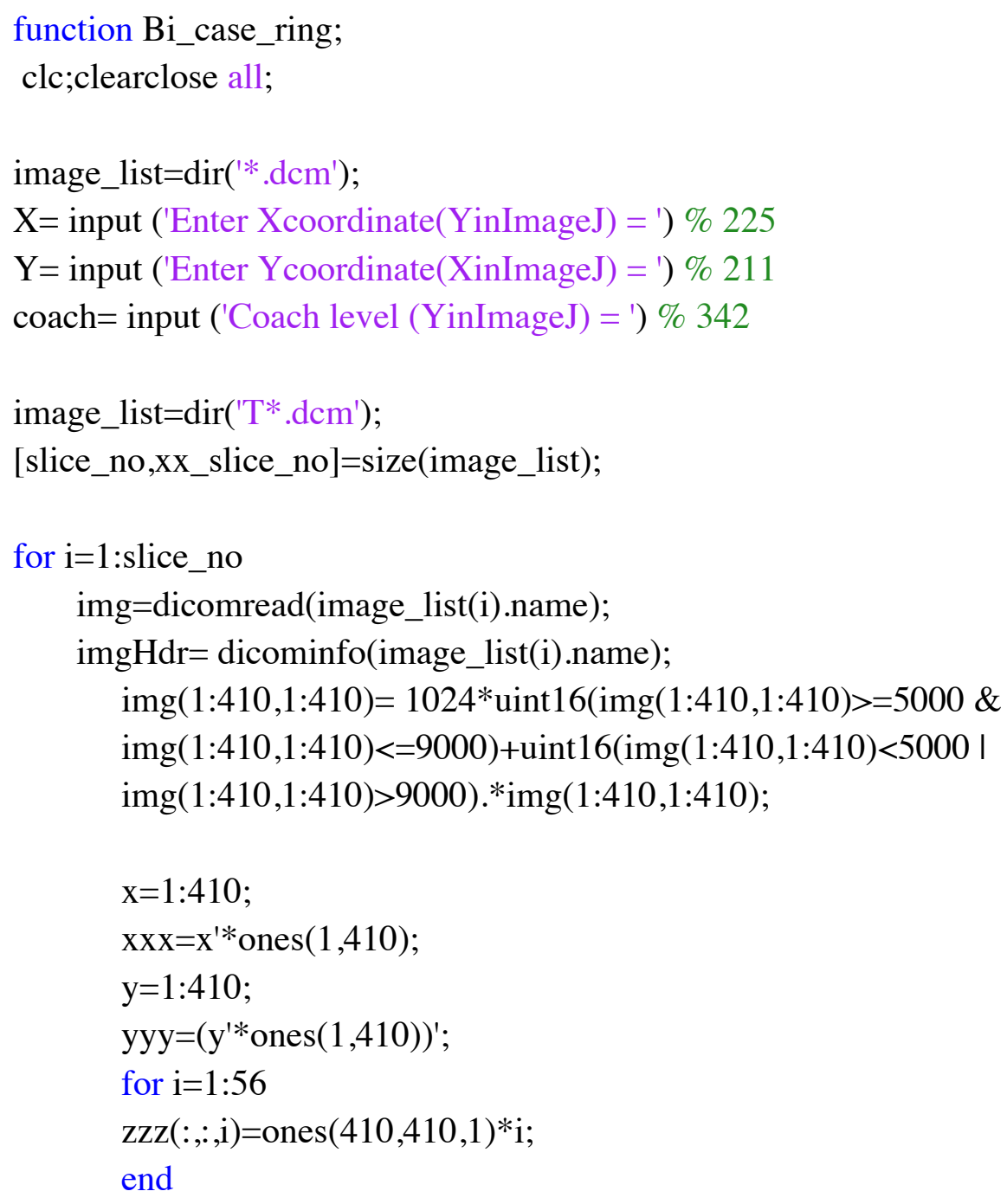


$\% * * * * * * * * * * * * * \%$ Ring $1 \mathrm{MLT} \% * * * * * * * * * * * * * \%$

$\%$ Finding threshold values for each material in this ring $x R i 1=\operatorname{size}\left(\operatorname{img}\left(\left(\left(\left((x x x-X) \cdot{ }^{\wedge} 2+(y y y-Y) .^{\wedge} 2\right)>0^{\wedge} 2\right) \&\left(\left((x x x-X) .^{\wedge} 2+(y y y-\right.\right.\right.\right.\right.$ Y).^2)<70^2))));

$x$ sizecbRi1 $=x \operatorname{Ri1}(1) * x \operatorname{Ri1}(2)$;

img_recb=reshape $\left(\operatorname{img}\left(\left(\left(\left(\right.\right.\right.\right.\right.$ xxx-X) $\left.\left..^{\wedge} 2+(y y y-Y) .^{\wedge} 2\right)>00^{\wedge} 2\right) \&\left(\left((x x x-X) \cdot^{\wedge} 2+(y y y-\right.\right.$ Y).^2)<70^2))),xsizecbRi1,1);

[hcbRi1,xcbRi1]=hist(double(img_recb),100);

[pixelsRi1,pvalue25Ri1]=max(hcbRi1(10:100));

HU_maxRi1=xcbRi1(pvalue25Ri1+9);

lo_MLT_hip=ceil(HU_maxRi1/0.3);

lo_MLT_bone=ceil(HU_maxRi1/0.785);

lo_MLT_h2o=ceil(HU_maxRi1/1.01);

lo_MLT_adipose=ceil(HU_maxRi1/1.5);

\%**************\% Ring $2 \mathrm{MLT} \% * * * * * * * * * * * * * \%$

\%Finding threshold values for each material in this ring $x \operatorname{Ri} 2=\operatorname{size}\left(\operatorname{img}\left(\left(\left(\left((x x x-X) .^{\wedge} 2+(y y y-Y) .^{\wedge} 2\right)>70^{\wedge} 2\right) \&\left(\left((x x x-X) .^{\wedge} 2+(y y y-\right.\right.\right.\right.\right.$ $\left.\left.\left.\left.\left.\mathrm{Y}) .^{\wedge} 2\right)<100^{\wedge} 2\right)\right)\right)\right)$; $x \operatorname{sizecbRi2}=x \operatorname{Ri} 2(1) * x \operatorname{Ri} 2(2)$;

img_recbRi2 $=\operatorname{reshape}\left(\operatorname{img}\left(\left(\left(\left((x x x-X) \cdot{ }^{\wedge} 2+(y y y-Y) \cdot{ }^{\wedge} 2\right)>70^{\wedge} 2\right) \&\left(\left((x x x-X) \cdot{ }^{\wedge} 2+(y y y-\right.\right.\right.\right.\right.$ Y).^2)<100^2))),xsizecbRi2,1);

[hcbRi2,xcbRi2]=hist(double(img_recbRi2),100);

[pixelsRi2,pvalue25Ri2]=max(hcbRi2(10:100));

HU_maxRi2=xcbRi2(pvalue25Ri2+9);

lo_MLT_hipRi2=ceil(HU_maxRi2/0.54);

lo_MLT_boneRi2=ceil(HU_maxRi2/0.8);

lo_MLT_h2oRi2=ceil(HU_maxRi2/1.0);

lo_MLT_adiposeRi2=floor(HU_maxRi2/HU_maxRi2^2);

$\% * * * * * * * * * * * * * \% \operatorname{Ring} 3 \mathrm{MLT} \% * * * * * * * * * * * * * \%$

$\%$ Finding threshold values for each material in this ring $x \operatorname{Ri3}=\operatorname{size}\left(\operatorname{img}\left(\left(\left(\left((x x x-X) \cdot{ }^{\wedge} 2+(y y y-Y) \cdot{ }^{\wedge} 2\right)>100^{\wedge} 2\right) \&\left(\left((x x x-X) \cdot{ }^{\wedge} 2+(y y y-\right.\right.\right.\right.\right.$ $\left.\left.\left.\left.\mathrm{Y}) .^{\wedge} 2\right)<140^{\wedge} 2\right)\right)\right)$ );

$x \operatorname{sizecbRi3}=x \operatorname{Ri3}(1) * x \operatorname{Ri3}(2)$;

img_recbRi3 $=$ reshape $\left(\operatorname{img}\left(\left(\left(\left((x x x-X) .^{\wedge} 2+(y y y-Y) \cdot{ }^{\wedge} 2\right)>100^{\wedge} 2\right) \&\left(\left((x x x-X) .^{\wedge} 2+(y y y-\right.\right.\right.\right.\right.$ Y). $\left.\left.\left.{ }^{\wedge} 2\right)<140^{\wedge} 2\right)\right)$ ), xsizecbRi3,1);

[hcbRi3,xcbRi3]=hist(double(img_recbRi3),100);

[pixelsRi3,pvalue25Ri3] =max(hcbRi3(10:100));

HU_maxRi3=xcbRi3(pvalue25Ri3+9);

lo_MLT_hipRi3=ceil(HU_maxRi3/0.54);

lo_MLT_boneRi3=ceil(HU_maxRi3/0.74);

lo_MLT_h2oRi3=ceil(HU_maxRi3/1.07); 
lo_MLT_adiposeRi3=ceil(HU_maxRi3/1.9);

$\% * * * * * * * * * * * * * \% \operatorname{Ring} 4 \mathrm{MLT} \% * * * * * * * * * * * * * \%$

$\%$ Finding threshold values for each material in this ring

$x R i 4=\operatorname{size}\left(\operatorname{img}\left(\left(\left((x x x-X) .^{\wedge} 2+(y y y-Y) .^{\wedge} 2\right)>140^{\wedge} 2\right) \&\left(\left((x x x-X) .^{\wedge} 2+(y y y-\right.\right.\right.\right.$

$\left.\left.\left.\left.\mathrm{Y}) .^{\wedge} 2\right)<170^{\wedge} 2\right)\right)\right)$ );

xsizecbRi4=xRi4(1)*xRi4(2);

img_recbRi4=reshape $\left(\operatorname{img}\left(\left(\left(\left(\right.\right.\right.\right.\right.$ xxx-X) $\left.\left..^{\wedge} 2+(y y y-Y) .^{\wedge} 2\right)>140^{\wedge} 2\right) \&\left(\left((x x x-X) .^{\wedge} 2+(y y y-\right.\right.$

Y).^2)<170^2))),xsizecbRi4,1);

[hcbRi4,xcbRi4]=hist(double(img_recbRi4),100);

[pixelsRi4,pvalue25Ri4]=max(hcbRi4(10:100));

HU_maxRi4=xcbRi4(pvalue25Ri4+9);

lo_MLT_hipRi4=ceil(HU_maxRi4/0.54);

lo_MLT_boneRi4=ceil(HU_maxRi4/0.75);

lo_MLT_h2oRi4=ceil(HU_maxRi4/1.1);

lo_MLT_adiposeRi4=ceil(HU_maxRi4/1.6);

$\% * * * * * * * * * * * * * \% \operatorname{Ring} 5 \mathrm{MLT} \% * * * * * * * * * * * * * \%$

$\%$ Finding threshold values for each material in this ring

$\mathrm{xRi5}=\operatorname{size}\left(\operatorname{img}\left(\left(\left(\left((\mathrm{xxx}-\mathrm{X}) \cdot{ }^{\wedge} 2+(\mathrm{yyy}-\mathrm{Y}) .^{\wedge} 2\right)>170^{\wedge} 2\right) \&\left(\left((\mathrm{xxx}-\mathrm{X}) .^{\wedge} 2+(\mathrm{yyy}-\right.\right.\right.\right.\right.$

Y).^2)<231^2))));

$x$ sizecbRi5 $=x \operatorname{Ri5}(1) * x \operatorname{Ri5}(2)$;

img_recbRi5=reshape $\left(\operatorname{img}\left(\left(\left(\left((x x x-X) .^{\wedge} 2+(y y y-Y) .^{\wedge} 2\right)>170^{\wedge} 2\right) \&(((x x x-\right.\right.\right.$

X).^2+(yyy-Y).^2)<231^2))),xsizecbRi5,1);

[hcbRi5,xcbRi5]=hist(double(img_recbRi5),100);

[pixelsRi5,pvalue25Ri5]=max(hcbRi5(4:100));

HU_maxRi5=xcbRi5(pvalue25Ri5+3);

if HU_maxRi5 <=140;

HU_maxRi5=156;

elseif HU_maxRi5 >=190;

HU_maxRi5=156;

else

HU_maxRi5=HU_maxRi5;

end

lo_MLT_h2oRi5=ceil(HU_maxRi5/0.25);

lo_MLT_adiposeRi5=ceil(HU_maxRi5/0.4);

$\% * * * * * * * * * * * * * * * * * * * * * * * * * * * * * \% \operatorname{Ring} 1 \% * * * * * * * * * * * * * * * * * * * * * * * * * * * * \%$

$\%$ Converting pixel values into HUs using the threshold values defined above for each material in this ring

$\operatorname{img}\left(\left(\left(\left((\mathrm{xxx}-\mathrm{X}) \cdot{ }^{\wedge} 2+(\mathrm{yyy}-\mathrm{Y}) \cdot^{\wedge} 2\right)>00^{\wedge} 2\right) \&\left(\left((\mathrm{xxx}-\mathrm{X}) \cdot^{\wedge} 2+(\mathrm{yyy}-\right.\right.\right.\right.$

$\left.\left.\left.\left.\mathrm{Y}) .^{\wedge} 2\right)<70^{\wedge} 2\right)\right)\right)=4000 * \operatorname{uint} 16\left(\operatorname{img}\left(\left(\left((\mathrm{xxx}-\mathrm{X}) \cdot{ }^{\wedge} 2+(\mathrm{yyy}-\mathrm{Y}) .^{\wedge} 2\right)>0^{\wedge} 2\right) \&(((\mathrm{xxx}-\right.\right.$ 
X).^2+(yyy-Y).^2)<70^2)) >=lo_MLT_hip \& img $\left(\left(\left((x x x-X) .^{\wedge} 2+(\right.\right.\right.$ yyy$\left.\left.\left.\left.\mathrm{Y}) .^{\wedge} 2\right)>0^{\wedge} 2\right) \&\left(\left((\mathrm{xxx}-\mathrm{X}) .^{\wedge} 2+(\mathrm{yyy}-\mathrm{Y}) .^{\wedge} 2\right)<70^{\wedge} 2\right)\right)<=9000\right)+\mathrm{uint} 16(\mathrm{img}(((((\mathrm{xxx}-$ $\left.\left.\left.\left.\mathrm{X}) .^{\wedge} 2+(\mathrm{yyy}-\mathrm{Y}) .^{\wedge} 2\right)>0^{\wedge} 2\right) \&\left(\left((\mathrm{xxx}-\mathrm{X}) \cdot{ }^{\wedge} 2+(\mathrm{yyy}-\mathrm{Y}) .^{\wedge} 2\right)<70^{\wedge} 2\right)\right)\right)<$ lo_MLT_hip I $\operatorname{img}\left(\left(\left(\left((x x x-X) .^{\wedge} 2+(y y y-Y) \cdot{ }^{\wedge} 2\right)>0^{\wedge} 2\right) \&\left(\left((x x x-X) \cdot^{\wedge} 2+(y y y-Y) .^{\wedge} 2\right)<70^{\wedge} 2\right)\right)\right.$ )$>9000) . * \operatorname{img}\left(\left(\left(\left((x x x-X) \wedge^{\wedge} 2+(y y y-Y) \cdot{ }^{\wedge} 2\right)>0 \wedge 2\right) \&\left(\left((x x x-X) \cdot{ }^{\wedge} 2+(y y y-Y) .^{\wedge} 2\right)<70^{\wedge} 2\right)\right)\right)$; $\operatorname{img}\left(\left(\left(\left((\mathrm{xxx}-\mathrm{X}) \cdot{ }^{\wedge} 2+(\mathrm{yyy}-\mathrm{Y}) \cdot^{\wedge} 2\right)>00^{\wedge} 2\right) \&\left(\left((\mathrm{xxx}-\mathrm{X}) \cdot^{\wedge} 2+(\mathrm{yyy}-\right.\right.\right.\right.$

$\left.\left.\left.\left.\mathrm{Y}) .^{\wedge} 2\right)<70^{\wedge} 2\right)\right)\right)=1552^{*} \operatorname{uint} 16\left(\operatorname{img}\left(\left(\left((\mathrm{xxx}-\mathrm{X}) .^{\wedge} 2+(\mathrm{yyy}-\mathrm{Y})\right)^{\wedge} 2\right)>0^{\wedge} 2\right) \&(((\mathrm{xxx}-\right.$ $\left.\left.\left.\mathrm{X}) .^{\wedge} 2+(\mathrm{yyy}-\mathrm{Y}) .^{\wedge} 2\right)<70^{\wedge} 2\right)\right)>=\mathrm{lo} \_M L T \_b o n e ~ \& \operatorname{img}\left(\left(\left((\mathrm{xxx}-\mathrm{X}) .^{\wedge} 2+(\mathrm{yyy}-\right.\right.\right.$ $\left.\left.\left.\mathrm{Y}) .^{\wedge} 2\right)>0^{\wedge} 2\right) \&\left(\left((\mathrm{xxx}-\mathrm{X}) .^{\wedge} 2+(\mathrm{yyy}-\mathrm{Y}) .^{\wedge} 2\right)<70^{\wedge} 2\right)\right)$

$<=$ lo_MLT_hip $)+$ uint16(img $\left(\left(\left(\left((\mathrm{xxx}-\mathrm{X}) .^{\wedge} 2+(\mathrm{yyy}-\mathrm{Y}) .^{\wedge} 2\right)>0^{\wedge} 2\right) \&\left(((\mathrm{xxx}-\mathrm{X}))^{\wedge} 2+(\mathrm{yyy}-\right.\right.\right.$ $\left.\left.\left.\left.\mathrm{Y}) .^{\wedge} 2\right)<70^{\wedge} 2\right)\right)\right)<$ lo_MLT_bone $\operatorname{limg}\left(\left(\left(\left((\mathrm{xxx}-\mathrm{X}) .^{\wedge} 2+(\mathrm{yyy}-\mathrm{Y}) .^{\wedge} 2\right)>0^{\wedge} 2\right) \&(((\mathrm{xxx}-\right.\right.$ $\left.\left.\left.\left.\left.\mathrm{X}) .^{\wedge} 2+(\mathrm{yyy}-\mathrm{Y}) .^{\wedge} 2\right)<70^{\wedge} 2\right)\right)\right)>\operatorname{lo} \_M L T \_h i p\right) . * \operatorname{img}\left(\left(\left(\left((\mathrm{xxx}-\mathrm{X}) .^{\wedge} 2+(\mathrm{yyy}-\right.\right.\right.\right.$ $\left.\left.\left.\left.\mathrm{Y}) .^{\wedge} 2\right)>0 \wedge 2\right) \&\left(\left((\mathrm{xxx}-\mathrm{X}) \cdot{ }^{\wedge} 2+(\mathrm{yyy}-\mathrm{Y}) .^{\wedge} 2\right)<70^{\wedge} 2\right)\right)\right)$; $\operatorname{img}\left(\left(\left(\left((\mathrm{xxx}-\mathrm{X}) \cdot{ }^{\wedge} 2+(\mathrm{yyy}-\mathrm{Y}) \cdot^{\wedge} 2\right)>00^{\wedge} 2\right) \&\left(\left((\mathrm{xxx}-\mathrm{X}) \cdot^{\wedge} 2+(\mathrm{yyy}-\right.\right.\right.\right.$ $\left.\left.\left.\left.\mathrm{Y}) .^{\wedge} 2\right)<70^{\wedge} 2\right)\right)\right)=1024 * \operatorname{uint} 16\left(\operatorname{img}\left(\left(\left((\mathrm{xxx}-\mathrm{X}) .^{\wedge} 2+(\mathrm{yyy}-\mathrm{Y}) .^{\wedge} 2\right)>0^{\wedge} 2\right) \&(((\mathrm{xxx}-\right.\right.$ $\left.\left.\left.\mathrm{X}) .^{\wedge} 2+(\mathrm{yyy}-\mathrm{Y}) .^{\wedge} 2\right)<70^{\wedge} 2\right)\right)>=\mathrm{lo} \_M L T \_h 20 \& \operatorname{img}\left(\left((\mathrm{xxx}-\mathrm{X}) .^{\wedge} 2+(\mathrm{yyy}-\right.\right.$ $\left.\left.\left.\mathrm{Y}) .^{\wedge} 2\right)>0^{\wedge} 2\right) \&\left(\left((\mathrm{xxx}-\mathrm{X}) \cdot^{\wedge} 2+(\mathrm{yyy}-\mathrm{Y}) .^{\wedge} 2\right)<70^{\wedge} 2\right)\right)$

$<=$ lo_MLT_bone $)+$ uint 16(img $\left(\left(\left(\left(\right.\right.\right.\right.$ xxx-X) $\left.\left..^{\wedge} 2+(\mathrm{yyy}-\mathrm{Y}) .^{\wedge} 2\right)>0^{\wedge} 2\right) \&\left(\left((\mathrm{xxx}-\mathrm{X}) .^{\wedge} 2+(\mathrm{yyy}-\right.\right.$ $\left.\left.\left.\left.\mathrm{Y}) .^{\wedge} 2\right)<70^{\wedge} 2\right)\right)\right)<\mathrm{lo} \_M L T \_h 2 o \mathrm{I} \operatorname{img}\left(\left(\left(((\mathrm{xxx}-\mathrm{X}))^{\wedge} 2+(\mathrm{yyy}-\mathrm{Y}) .^{\wedge} 2\right)>00^{\wedge} 2\right) \&(((\mathrm{xxx}-\right.$

$\left.\left.\left.\left.\left.\mathrm{X}) .^{\wedge} 2+(\mathrm{yyy}-\mathrm{Y}) .^{\wedge} 2\right)<70^{\wedge} 2\right)\right)\right)>\operatorname{lo} \_M L T \_b o n e\right) . * \operatorname{img}\left(\left(\left((\mathrm{xxx}-\mathrm{X}) .^{\wedge} 2+(\mathrm{yyy}-\right.\right.\right.$ $\left.\left.\left.\left.\mathrm{Y}) .^{\wedge} 2\right)>0^{\wedge} 2\right) \&\left(\left((\mathrm{xxx}-\mathrm{X}) \cdot{ }^{\wedge} 2+(\mathrm{yyy}-\mathrm{Y}) .^{\wedge} 2\right)<70^{\wedge} 2\right)\right)\right)$; $\operatorname{img}\left(\left(\left((\mathrm{xxx}-\mathrm{X}) \cdot{ }^{\wedge} 2+(\mathrm{yyy}-\mathrm{Y}) \cdot^{\wedge} 2\right)>00^{\wedge} 2\right) \&\left(\left((\mathrm{xxx}-\mathrm{X}) \cdot{ }^{\wedge} 2+(\mathrm{yyy}-\right.\right.\right.$ $\left.\left.\left.\left.\mathrm{Y}) .^{\wedge} 2\right)<70^{\wedge} 2\right)\right)\right)=928^{*} \operatorname{uint} 16\left(\operatorname{img}\left(\left(\left((\mathrm{xxx}-\mathrm{X}) .^{\wedge} 2+(\mathrm{yyy}-\mathrm{Y}) .^{\wedge} 2\right)>0^{\wedge} 2\right) \&(((\mathrm{xxx}-\right.\right.$ $\left.\left.\left.\mathrm{X}) .^{\wedge} 2+(\mathrm{yyy}-\mathrm{Y}) .^{\wedge} 2\right)<70^{\wedge} 2\right)\right)>=\mathrm{lo} \_M L T \_a d i p o s e ~ \& \operatorname{img}\left(\left(\left((\mathrm{xxx}-\mathrm{X}) \cdot{ }^{\wedge} 2+(\mathrm{yyy}-\right.\right.\right.$ $\left.\left.\left.\mathrm{Y}) .^{\wedge} 2\right)>0^{\wedge} 2\right) \&\left(\left((\mathrm{xxx}-\mathrm{X}) .^{\wedge} 2+(\mathrm{yyy}-\mathrm{Y}) .^{\wedge} 2\right)<70^{\wedge} 2\right)\right)$

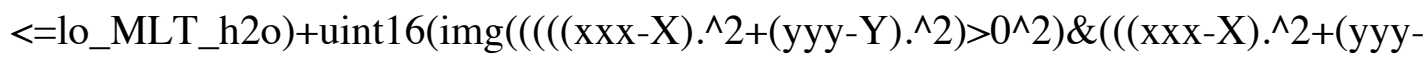
$\left.\left.\left.\left.Y) .^{\wedge} 2\right)<70^{\wedge} 2\right)\right)\right)<$ lo_MLT_adipose $I \operatorname{img}\left(\left(\left(\left((x x x-X) .^{\wedge} 2+(y y y-Y) .^{\wedge} 2\right)>0^{\wedge} 2\right) \&(((x x x-\right.\right.$ $\left.\left.\left.\left.\mathrm{X}) .^{\wedge} 2+(\mathrm{yyy}-\mathrm{Y}) .^{\wedge} 2\right)<70^{\wedge} 2\right)\right)\right)>$ lo_MLT_h2o $) . * \operatorname{img}\left(\left(\left(\left((\mathrm{xxx}-\mathrm{X}) .^{\wedge} 2+(\mathrm{yyy}-\right.\right.\right.\right.$ $\left.\left.\left.\left.\mathrm{Y}) .^{\wedge} 2\right)>0 \wedge 2\right) \&\left(\left((\mathrm{xxx}-\mathrm{X}) .^{\wedge} 2+(\mathrm{yyy}-\mathrm{Y}) .^{\wedge} 2\right)<70^{\wedge} 2\right)\right)\right)$;

$\% * * * * * * * * * * * * * * * * * * * * * * * * * * * * * \% \mathrm{Ring} 2 \% * * * * * * * * * * * * * * * * * * * * * * * * * * * * \%$ $\%$ Converting pixel values into HUs using the threshold values defined above for each material in this ring $\operatorname{img}\left(\left(\left(\left((\mathrm{xxx}-\mathrm{X}) \cdot{ }^{\wedge} 2+(\mathrm{yyy}-\mathrm{Y}) .^{\wedge} 2\right)>70^{\wedge} 2\right) \&\left(\left((\mathrm{xxx}-\mathrm{X}) .^{\wedge} 2+(\mathrm{yyy}-\right.\right.\right.\right.$ $\left.\left.\left.\left.\mathrm{Y}) .^{\wedge} 2\right)<100^{\wedge} 2\right)\right)\right)=4000^{*} \operatorname{uint} 16\left(\operatorname{img}\left(\left(\left((\mathrm{xxx}-\mathrm{X}) .^{\wedge} 2+(\mathrm{yyy}-\mathrm{Y}) .^{\wedge} 2\right)>70^{\wedge} 2\right) \&(((\mathrm{xxx}-\right.\right.$ $\left.\left.\left.\mathrm{X}) .^{\wedge} 2+(\mathrm{yyy}-\mathrm{Y}) . \wedge 2\right)<100^{\wedge} 2\right)\right)>=\mathrm{lo} \_M L T \_h i p R i 2 \& \operatorname{img}\left(\left((\mathrm{xxx}-\mathrm{X}) .^{\wedge} 2+(\mathrm{yyy}-\right.\right.$ $\left.\left.\left.\left.\mathrm{Y}) .^{\wedge} 2\right)>70^{\wedge} 2\right) \&\left(\left((\mathrm{xxx}-\mathrm{X}) .^{\wedge} 2+(\mathrm{yyy}-\mathrm{Y}) .^{\wedge} 2\right)<100^{\wedge} 2\right)\right)<=9000\right)+\mathrm{uint} 16(\mathrm{img}(((((\mathrm{xxx}-$ $\left.\left.\left.\left.\mathrm{X}) .^{\wedge} 2+(\mathrm{yyy}-\mathrm{Y}) \cdot{ }^{\wedge} 2\right)>70^{\wedge} 2\right) \&\left(\left((\mathrm{xxx}-\mathrm{X}) \cdot{ }^{\wedge} 2+(\mathrm{yyy}-\mathrm{Y}) \cdot{ }^{\wedge} 2\right)<100^{\wedge} 2\right)\right)\right)<$ lo_MLT_hipRi2 । $\operatorname{img}\left(\left(\left(\left((\mathrm{xxx}-\mathrm{X}) \cdot{ }^{\wedge} 2+(\mathrm{yyy}-\mathrm{Y}) \cdot{ }^{\wedge} 2\right)>70^{\wedge} 2\right) \&\left(\left((\mathrm{xxx}-\mathrm{X}) \cdot{ }^{\wedge} 2+(\mathrm{yyy}-\mathrm{Y}) \cdot{ }^{\wedge} 2\right)<100^{\wedge} 2\right)\right)\right.$ )$>9000) . * \operatorname{img}\left(\left(\left(\left((x x x-X) \wedge^{\wedge} 2+(y y y-Y) .^{\wedge} 2\right)>70^{\wedge} 2\right) \&\left(\left((x x x-X) .^{\wedge} 2+(y y y-Y) .^{\wedge} 2\right)<100^{\wedge} 2\right)\right)\right.$ )

$\operatorname{img}\left(\left(\left((\mathrm{xxx}-\mathrm{X}) \cdot{ }^{\wedge} 2+(\mathrm{yyy}-\mathrm{Y}) \cdot{ }^{\wedge} 2\right)>70^{\wedge} 2\right) \&\left(\left((\mathrm{xxx}-\mathrm{X}) .^{\wedge} 2+(\mathrm{yyy}-\right.\right.\right.$ $\left.\left.\left.\left.\mathrm{Y}) .^{\wedge} 2\right)<100^{\wedge} 2\right)\right)\right)=1552^{*} \operatorname{uint} 16\left(\operatorname{img}\left(\left(\left((\mathrm{xxx}-\mathrm{X}) .^{\wedge} 2+(\mathrm{yyy}-\mathrm{Y}) .^{\wedge} 2\right)>70^{\wedge} 2\right) \&(((\mathrm{xxx}-\right.\right.$ 
$\left.\left.\left.\mathrm{X}) .^{\wedge} 2+(\mathrm{yyy}-\mathrm{Y}) .^{\wedge} 2\right)<100^{\wedge} 2\right)\right)>=\mathrm{lo} \_M L T \_b o n e R i 2 \& \operatorname{img}\left(\left(\left((\mathrm{xxx}-\mathrm{X}) .^{\wedge} 2+(\mathrm{yyy}-\right.\right.\right.$ $\left.\left.\left.\mathrm{Y}) .^{\wedge} 2\right)>70^{\wedge} 2\right) \&\left(\left((\mathrm{xxx}-\mathrm{X}) .^{\wedge} 2+(\mathrm{yyy}-\mathrm{Y}) .{ }^{\wedge} 2\right)<100^{\wedge} 2\right)\right)$

$<=$ lo_MLT_hipRi2)+uint16(img $\left(\left(\left(\left((\mathrm{xxx}-\mathrm{X}) .^{\wedge} 2+(\mathrm{yyy}-\mathrm{Y}) .^{\wedge} 2\right)>70^{\wedge} 2\right) \&(((\mathrm{xxx}-\right.\right.$ $\left.\left.\left.\left.\mathrm{X}) .^{\wedge} 2+(\mathrm{yyy}-\mathrm{Y}) .^{\wedge} 2\right)<100^{\wedge} 2\right)\right)\right)<\mathrm{lo} \_M L T \_b o n e R i 2 \operatorname{img}\left(\left(\left(\left((\mathrm{xxx}-\mathrm{X}) .^{\wedge} 2+(\mathrm{yyy}-\right.\right.\right.\right.$ $\left.\left.\left.\left.\mathrm{Y}) .^{\wedge} 2\right)>70^{\wedge} 2\right) \&\left(\left((\mathrm{xxx}-\mathrm{X}) .^{\wedge} 2+(\mathrm{yyy}-\mathrm{Y}) .^{\wedge} 2\right)<100^{\wedge} 2\right)\right)\right)>$ lo_MLT_hipRi2).*img $(((((\mathrm{xxx}-$ $\left.\left.\left.\left.\mathrm{X}) .^{\wedge} 2+(\mathrm{yyy}-\mathrm{Y}) .^{\wedge} 2\right)>70^{\wedge} 2\right) \&\left(\left((\mathrm{xxx}-\mathrm{X}) .^{\wedge} 2+(\mathrm{yyy}-\mathrm{Y}) .^{\wedge} 2\right)<100^{\wedge} 2\right)\right)\right)$;

$\operatorname{img}\left(\left(\left(\left((\mathrm{xxx}-\mathrm{X}) .^{\wedge} 2+(\mathrm{yyy}-\mathrm{Y}) .^{\wedge} 2\right)>70^{\wedge} 2\right) \&\left(\left((\mathrm{xxx}-\mathrm{X}) .^{\wedge} 2+(\mathrm{yyy}-\right.\right.\right.\right.$

Y). $\left.\left.\left.\left.\wedge^{\wedge}\right)<100^{\wedge} 2\right)\right)\right)=1024^{*} \operatorname{uint} 16\left(\operatorname{img}\left(\left(\left((x x x-X) .^{\wedge} 2+(y y y-Y) .^{\wedge} 2\right)>70^{\wedge} 2\right) \&(((x x x-\right.\right.$ $\left.\left.\left.\mathrm{X}) .^{\wedge} 2+(\mathrm{yyy}-\mathrm{Y}) .^{\wedge} 2\right)<100^{\wedge} 2\right)\right)>=\mathrm{lo} \_M L T \_h 20 R i 2 \& \operatorname{img}\left(\left(\left((\mathrm{xxx}-\mathrm{X}) .^{\wedge} 2+(\mathrm{yyy}-\right.\right.\right.$ $\left.\left.\left.\mathrm{Y}) .^{\wedge} 2\right)>70^{\wedge} 2\right) \&\left(\left((\mathrm{xxx}-\mathrm{X}) .^{\wedge} 2+(\mathrm{yyy}-\mathrm{Y}) .{ }^{\wedge} 2\right)<100^{\wedge} 2\right)\right)$

$<=$ lo_MLT_boneRi2)+uint16(img $\left(\left(\left(\left((\mathrm{xxx}-\mathrm{X}) .^{\wedge} 2+(\mathrm{yyy}-\mathrm{Y}) \cdot{ }^{\wedge} 2\right)>70^{\wedge} 2\right) \&(((\mathrm{xxx}-\right.\right.$ $\left.\left.\left.\left.\mathrm{X}) .^{\wedge} 2+(\mathrm{yyy}-\mathrm{Y}) . \wedge 2\right)<100^{\wedge} 2\right)\right)\right)<\mathrm{lo} \_M L T \_h 20 R i 2 \mathrm{Img}\left(\left(\left((\mathrm{xxx}-\mathrm{X}) \cdot{ }^{\wedge} 2+(\mathrm{yyy}-\right.\right.\right.$ $\left.\left.\left.\mathrm{Y}) .^{\wedge} 2\right)>70^{\wedge} 2\right) \&\left(\left((\mathrm{xxx}-\mathrm{X}) .^{\wedge} 2+(\mathrm{yyy}-\mathrm{Y}) . \wedge 2\right)<100^{\wedge} 2\right)\right)$

)$>$ lo_MLT_boneRi2).*img $\left(\left(\left(\left((x x x-X) .^{\wedge} 2+(y y y-Y) \wedge^{\wedge} 2\right)>70^{\wedge} 2\right) \&\left(\left((x x x-X) .^{\wedge} 2+(y y y-\right.\right.\right.\right.$ Y).^2)<100^2)) );

$\operatorname{img}\left(\left(\left((\mathrm{xxx}-\mathrm{X}) \cdot \cdot^{\wedge} 2+(\mathrm{yyy}-\mathrm{Y}) \cdot^{\wedge} 2\right)>70^{\wedge} 2\right) \&\left(\left((\mathrm{xxx}-\mathrm{X}) \cdot^{\wedge} 2+(\mathrm{yyy}-\right.\right.\right.$

$\left.\left.\left.\left.\mathrm{Y}) .^{\wedge} 2\right)<100^{\wedge} 2\right)\right)\right)=928^{*} \operatorname{uint} 16\left(\operatorname{img}\left(\left(\left((\mathrm{xxx}-\mathrm{X}) .^{\wedge} 2+(\mathrm{yyy}-\mathrm{Y}) .^{\wedge} 2\right)>70^{\wedge} 2\right) \&(((\mathrm{xxx}-\right.\right.$

$\left.\left.\left.\mathrm{X}) .^{\wedge} 2+(\mathrm{yyy}-\mathrm{Y}) .{ }^{\wedge} 2\right)<100^{\wedge} 2\right)\right)>=\mathrm{lo} \_M L T \_a d i p o s e R i 2 \& \operatorname{img}\left(\left((\mathrm{xxx}-\mathrm{X}) .^{\wedge} 2+(\mathrm{yyy}-\right.\right.$ $\left.\left.\mathrm{Y}) . \wedge 2)>70^{\wedge} 2\right) \&\left(\left((\mathrm{xxx}-\mathrm{X}) \cdot{ }^{\wedge} 2+(\mathrm{yyy}-\mathrm{Y}) . \wedge 2\right)<100^{\wedge} 2\right)\right)$

$<=$ lo_MLT_h2oRi2)+uint16(img $(((($ xxx-X).^2+(yyy-Y).^2)>70^2)\&(((xxx$\left.\left.\left.\left.\mathrm{X}) .^{\wedge} 2+(\mathrm{yyy}-\mathrm{Y}) .{ }^{\wedge} 2\right)<100^{\wedge} 2\right)\right)\right)<\mathrm{lo} \_M L T \_a d i p o s e R i 2 \operatorname{img}\left(\left(\left((\mathrm{xxx}-\mathrm{X}) .^{\wedge} 2+(\mathrm{yyy}-\right.\right.\right.$ $\left.\left.\left.\left.\mathrm{Y}) .^{\wedge} 2\right)>70^{\wedge} 2\right) \&\left(\left((\mathrm{xxx}-\mathrm{X}) .^{\wedge} 2+(\mathrm{yyy}-\mathrm{Y}) .^{\wedge} 2\right)<100^{\wedge} 2\right)\right)\right)>$ lo_MLT_h2oRi2).*img $(((((\mathrm{xxx}-$ $\left.\left.\left.\left.\mathrm{X}) .^{\wedge} 2+(\mathrm{yyy}-\mathrm{Y}) .^{\wedge} 2\right)>70^{\wedge} 2\right) \&\left(\left((\mathrm{xxx}-\mathrm{X}) \cdot{ }^{\wedge} 2+(\mathrm{yyy}-\mathrm{Y}) \cdot{ }^{\wedge} 2\right)<100^{\wedge} 2\right)\right)\right)$;

$\% * * * * * * * * * * * * * * * * * * * * * * * * * * * * * \% \operatorname{Ring} 3 \% * * * * * * * * * * * * * * * * * * * * * * * * * * * * \%$ $\%$ Converting pixel values into HUs using the threshold values defined above for each material in this ring $\operatorname{img}\left(\left(\left(\left(\right.\right.\right.\right.$ xxx-X).^2+(yyy-Y) $\left.\left.\cdot^{\wedge} 2\right)>100^{\wedge} 2\right) \&\left(\left((x x x-X) \cdot{ }^{\wedge} 2+(y y y-\right.\right.$ $\left.\left.\left.\left.\mathrm{Y}) .^{\wedge} 2\right)<140^{\wedge} 2\right)\right)\right)=4000 * \operatorname{uint} 16\left(\operatorname{img}\left(\left(\left((\mathrm{xxx}-\mathrm{X}) .^{\wedge} 2+(\mathrm{yyy}-\mathrm{Y}) .^{\wedge} 2\right)>100^{\wedge} 2\right) \&(((\mathrm{xxx}-\right.\right.$ $\left.\left.\left.\mathrm{X}) .^{\wedge} 2+(\mathrm{yyy}-\mathrm{Y}) .^{\wedge} 2\right)<140^{\wedge} 2\right)\right)>=\mathrm{lo} \_M L T \_h i p R i 3 \& \operatorname{img}\left(\left((\mathrm{xxx}-\mathrm{X}) .^{\wedge} 2+(\mathrm{yyy}-\right.\right.$ $\left.\left.\left.\left.\mathrm{Y}) .^{\wedge} 2\right)>100^{\wedge} 2\right) \&\left(\left((\mathrm{xxx}-\mathrm{X}) .^{\wedge} 2+(\mathrm{yyy}-\mathrm{Y}) .{ }^{\wedge} 2\right)<140^{\wedge} 2\right)\right)<=9000\right)+\mathrm{uint} 16(\mathrm{img}((((\mathrm{xxx}-$ $\left.\left.\left.\left.X) .^{\wedge} 2+(y y y-Y) . \wedge 2\right)>100^{\wedge} 2\right) \&\left(\left((x x x-X) \cdot{ }^{\wedge} 2+(y y y-Y) . \wedge 2\right)<140^{\wedge} 2\right)\right)\right)<$ lo_MLT_hipRi3 I $\operatorname{img}\left(\left(\left(\left((\mathrm{xxx}-\mathrm{X}) .^{\wedge} 2+(\mathrm{yyy}-\mathrm{Y}) .^{\wedge} 2\right)>100^{\wedge} 2\right) \&\left(\left((\mathrm{xxx}-\mathrm{X}) .^{\wedge} 2+(\mathrm{yyy}-\mathrm{Y}) .^{\wedge} 2\right)<140^{\wedge} 2\right)\right)\right.$ )$>9000) \cdot * \operatorname{img}\left(\left(\left(\left((\mathrm{xxx}-\mathrm{X}) \cdot^{\wedge} 2+(\mathrm{yyy}-\mathrm{Y}) .^{\wedge} 2\right)>100^{\wedge} 2\right) \&\left(\left((\mathrm{xxx}-\mathrm{X}) \cdot{ }^{\wedge} 2+(\mathrm{yyy}-\right.\right.\right.\right.$ $\left.\left.\left.\left.\mathrm{Y}) .^{\wedge} 2\right)<140^{\wedge} 2\right)\right)\right)$; $\operatorname{img}\left(\left(\left(\left((\mathrm{xxx}-\mathrm{X}) \cdot{ }^{\wedge} 2+(\mathrm{yyy}-\mathrm{Y}) \cdot{ }^{\wedge} 2\right)>100^{\wedge} 2\right) \&\left(\left((\mathrm{xxx}-\mathrm{X}) \cdot{ }^{\wedge} 2+(\mathrm{yyy}-\right.\right.\right.\right.$ $\left.\left.\left.\left.\mathrm{Y}) .^{\wedge} 2\right)<140^{\wedge} 2\right)\right)\right)=1552 * \operatorname{uint} 16\left(\operatorname{img}\left(\left(\left((\mathrm{xxx}-\mathrm{X}) .^{\wedge} 2+(\mathrm{yyy}-\mathrm{Y}) .^{\wedge} 2\right)>100^{\wedge} 2\right) \&(((\mathrm{xxx}-\right.\right.$ $\left.\left.\left.\mathrm{X}) .^{\wedge} 2+(\mathrm{yyy}-\mathrm{Y}) \cdot{ }^{\wedge} 2\right)<140^{\wedge} 2\right)\right)>=$ lo_MLT_boneRi3 \& img $\left(\left(\left((\mathrm{xxx}-\mathrm{X}) \cdot{ }^{\wedge} 2+(\mathrm{yyy}-\right.\right.\right.$ $\left.\left.\left.\mathrm{Y}) .^{\wedge} 2\right)>100^{\wedge} 2\right) \&\left(\left((\mathrm{xxx}-\mathrm{X}) \cdot{ }^{\wedge} 2+(\mathrm{yyy}-\mathrm{Y}) \cdot{ }^{\wedge} 2\right)<140^{\wedge} 2\right)\right)$

$<=$ lo_MLT_hipRi3)+uint16(img $\left(\left(\left(((x x x-X))^{\wedge} 2+(y y y-Y) .^{\wedge} 2\right)>100^{\wedge} 2\right) \&(((x x x-\right.$ $\left.\left.\left.\left.\mathrm{X}) .^{\wedge} 2+(\mathrm{yyy}-\mathrm{Y}) .{ }^{\wedge} 2\right)<140^{\wedge} 2\right)\right)\right)<\mathrm{lo} \_M L T \_b o n e R i 3 \mathrm{I} \operatorname{img}\left(\left(\left((\mathrm{xxx}-\mathrm{X}) .^{\wedge} 2+(\mathrm{yyy}-\right.\right.\right.$ $\left.\left.\left.\mathrm{Y}) .^{\wedge} 2\right)>100^{\wedge} 2\right) \&\left(\left((\mathrm{xxx}-\mathrm{X}) .^{\wedge} 2+(\mathrm{yyy}-\mathrm{Y}) \cdot{ }^{\wedge} 2\right)<140^{\wedge} 2\right)\right)$ )$\left.>10 \_M L T \_h i p R i 3\right) . * \operatorname{img}\left(\left(\left((\mathrm{xxx}-\mathrm{X}) \cdot{ }^{\wedge} 2+(\mathrm{yyy}-\mathrm{Y}) .^{\wedge} 2\right)>100^{\wedge} 2\right) \&\left(\left((\mathrm{xxx}-\mathrm{X}) \cdot{ }^{\wedge} 2+(\mathrm{yyy}-\right.\right.\right.$ 
Y).^2)<140^2)) );

$\operatorname{img}\left(\left(\left(\left((\mathrm{xxx}-\mathrm{X}) \cdot^{\wedge} 2+(\mathrm{yyy}-\mathrm{Y}) .^{\wedge} 2\right)>100^{\wedge} 2\right) \&\left(\left((\mathrm{xxx}-\mathrm{X}) \cdot{ }^{\wedge} 2+(\mathrm{yyy}-\right.\right.\right.\right.$

$\left.\left.\left.\left.\mathrm{Y}) .^{\wedge} 2\right)<140^{\wedge} 2\right)\right)\right)=1024 * \operatorname{uint} 16\left(\operatorname{img}\left(\left(\left((\mathrm{xxx}-\mathrm{X}) .^{\wedge} 2+(\mathrm{yyy}-\mathrm{Y}) .^{\wedge} 2\right)>100^{\wedge} 2\right) \&(((\mathrm{xxx}-\right.\right.$

$\left.\left.\left.\mathrm{X}) .^{\wedge} 2+(\mathrm{yyy}-\mathrm{Y}) .^{\wedge} 2\right)<140^{\wedge} 2\right)\right)>=\mathrm{lo} \_M L T \_h 20 R i 3 \& \operatorname{img}\left(\left(\left((\mathrm{xxx}-\mathrm{X}) .^{\wedge} 2+(\mathrm{yyy}-\right.\right.\right.$

$\left.\left.\left.\mathrm{Y}) .^{\wedge} 2\right)>100^{\wedge} 2\right) \&\left(\left((\mathrm{xxx}-\mathrm{X}) .^{\wedge} 2+(\mathrm{yyy}-\mathrm{Y}) \cdot{ }^{\wedge} 2\right)<140^{\wedge} 2\right)\right)$

$<=$ lo_MLT_boneRi3)+uint16(img $(((($ xxx-X).^2+(yyy-Y).^2)>100^2)\&(((xxx-

$\left.\left.\left.\left.\mathrm{X}) .^{\wedge} 2+(\mathrm{yyy}-\mathrm{Y}) .^{\wedge} 2\right)<140^{\wedge} 2\right)\right)\right)<\mathrm{lo} \_M L T \_h 20 R i 3 \mathrm{I} \operatorname{img}\left(\left(\left(\left((\mathrm{xxx}-\mathrm{X}) .^{\wedge} 2+(\mathrm{yyy}-\right.\right.\right.\right.$

$\left.\left.\left.\mathrm{Y}) .^{\wedge} 2\right)>100^{\wedge} 2\right) \&\left(\left((\mathrm{xxx}-\mathrm{X}) .^{\wedge} 2+(\mathrm{yyy}-\mathrm{Y}) .^{\wedge} 2\right)<140^{\wedge} 2\right)\right)$

)$>$ lo_MLT_boneRi3).*img $\left(\left(\left((\mathrm{xxx}-\mathrm{X}) .^{\wedge} 2+(\mathrm{yyy}-\mathrm{Y}) .^{\wedge} 2\right)>100^{\wedge} 2\right) \&\left(\left((\mathrm{xxx}-\mathrm{X}) .^{\wedge} 2+(\mathrm{yyy}-\right.\right.\right.$ Y).^2)<140^2)) ;

$\operatorname{img}\left(\left(\left(\left((\mathrm{xxx}-\mathrm{X}) \cdot^{\wedge} 2+(\mathrm{yyy}-\mathrm{Y}) \cdot^{\wedge} 2\right)>100^{\wedge} 2\right) \&\left(\left((\mathrm{xxx}-\mathrm{X}) \cdot{ }^{\wedge} 2+(\mathrm{yyy}-\right.\right.\right.\right.$

$\left.\left.\left.\left.\mathrm{Y}) .^{\wedge} 2\right)<140^{\wedge} 2\right)\right)\right)=928 * \operatorname{uint} 16\left(\operatorname{img}\left(\left(\left((\mathrm{xxx}-\mathrm{X}) .^{\wedge} 2+(\mathrm{yyy}-\mathrm{Y}) \cdot{ }^{\wedge} 2\right)>100^{\wedge} 2\right) \&(((\mathrm{xxx}-\right.\right.$

$\left.\left.\left.\mathrm{X}) .^{\wedge} 2+(\mathrm{yyy}-\mathrm{Y}) .{ }^{\wedge} 2\right)<140^{\wedge} 2\right)\right)>=\mathrm{lo} \_M L T \_a d i p o s e R i 3 \& \operatorname{img}\left(\left(\left((\mathrm{xxx}-\mathrm{X}) .^{\wedge} 2+(\mathrm{yyy}-\right.\right.\right.$

$\left.\left.\left.\mathrm{Y}) .^{\wedge} 2\right)>100^{\wedge} 2\right) \&\left(\left((\mathrm{xxx}-\mathrm{X}) \cdot^{\wedge} 2+(\mathrm{yyy}-\mathrm{Y}) \cdot{ }^{\wedge} 2\right)<140^{\wedge} 2\right)\right)$

$<=$ lo_MLT_h2oRi3)+uint16(img $(((($ xxx-X).^2+(yyy-Y).^2)>100^2) \& $(((x x x-$

$\left.\left.\left.\left.\mathrm{X}) .^{\wedge} 2+(\mathrm{yyy}-\mathrm{Y}) . \wedge 2\right)<140^{\wedge} 2\right)\right)\right)<\mathrm{lo} \_M L T \_a d i p o s e R i 3 \mathrm{I} \operatorname{img}\left(\left(\left((\mathrm{xxx}-\mathrm{X}) .^{\wedge} 2+(\mathrm{yyy}-\right.\right.\right.$

$\left.\left.\left.\mathrm{Y}) .^{\wedge} 2\right)>100^{\wedge} 2\right) \&\left(\left((\mathrm{xxx}-\mathrm{X}) .^{\wedge} 2+(\mathrm{yyy}-\mathrm{Y}) \cdot{ }^{\wedge} 2\right)<140^{\wedge} 2\right)\right)$

)$>$ lo_MLT_h2oRi3).*img $\left(\left(\left((\mathrm{xxx}-\mathrm{X}) .^{\wedge} 2+(\mathrm{yyy}-\mathrm{Y}) .^{\wedge} 2\right)>100^{\wedge} 2\right) \&\left(\left((\mathrm{xxx}-\mathrm{X}) .^{\wedge} 2+(\mathrm{yyy}-\right.\right.\right.$

Y).^2)<140^2)) );

$\% * * * * * * * * * * * * * * * * * * * * * * * * * * * * * \% \mathrm{Ring} 4 \% * * * * * * * * * * * * * * * * * * * * * * * * * * * * \%$

$\%$ Converting pixel values into HUs using the threshold values defined above for each material in this ring

$\operatorname{img}\left(\left(\left(\left((\mathrm{xxx}-\mathrm{X}) \cdot{ }^{\wedge} 2+(\mathrm{yyy}-\mathrm{Y}) \cdot{ }^{\wedge} 2\right)>140^{\wedge} 2\right) \&\left(\left((\mathrm{xxx}-\mathrm{X}) \cdot{ }^{\wedge} 2+(\mathrm{yyy}-\right.\right.\right.\right.$

$\left.\left.\left.\left.\mathrm{Y}) .^{\wedge} 2\right)<170^{\wedge} 2\right)\right)\right)=4000^{*} \operatorname{uint} 16\left(\operatorname{img}\left(\left(\left((\mathrm{xxx}-\mathrm{X}) .^{\wedge} 2+(\mathrm{yyy}-\mathrm{Y}) \cdot{ }^{\wedge} 2\right)>140^{\wedge} 2\right) \&(((\mathrm{xxx}-\right.\right.$

$\left.\left.\left.\mathrm{X}) .^{\wedge} 2+(\mathrm{yyy}-\mathrm{Y}) .^{\wedge} 2\right)<170^{\wedge} 2\right)\right)>=\mathrm{lo} \_M L T \_h i p R i 4 \& \operatorname{img}\left(\left(\left((\mathrm{xxx}-\mathrm{X}) \cdot{ }^{\wedge} 2+(\mathrm{yyy}-\right.\right.\right.$

$\left.\left.\left.\left.\mathrm{Y}) .^{\wedge} 2\right)>140^{\wedge} 2\right) \&\left(\left((\mathrm{xxx}-\mathrm{X}) .^{\wedge} 2+(\mathrm{yyy}-\mathrm{Y}) .^{\wedge} 2\right)<170^{\wedge} 2\right)\right)<=9000\right)+\mathrm{uint} 16(\mathrm{img}(((((\mathrm{xxx}-$

$\left.\left.\left.\left.\mathrm{X}) .^{\wedge} 2+(\mathrm{yyy}-\mathrm{Y}) .^{\wedge} 2\right)>140^{\wedge} 2\right) \&\left(\left((\mathrm{xxx}-\mathrm{X}) .^{\wedge} 2+(\mathrm{yyy}-\mathrm{Y}) .^{\wedge} 2\right)<170^{\wedge} 2\right)\right)\right)<$ lo_MLT_hipRi4 I

$\operatorname{img}\left(\left(\left(((x x x-X))^{\wedge} 2+(y y y-Y) .^{\wedge} 2\right)>140^{\wedge} 2\right) \&\left(\left((x x x-X) \wedge^{\wedge} 2+(y y y-Y) .^{\wedge} 2\right)<170^{\wedge} 2\right)\right)$

)$>9000) \cdot * \operatorname{img}\left(\left(\left(\left((\mathrm{xxx}-\mathrm{X}) \cdot^{\wedge} 2+(\mathrm{yyy}-\mathrm{Y}) .^{\wedge} 2\right)>140^{\wedge} 2\right) \&\left(((\mathrm{xxx}-\mathrm{X}))^{\wedge} 2+(\mathrm{yyy}-\right.\right.\right.$

$\left.\left.\left.\left.\mathrm{Y}) \wedge^{\wedge} 2\right)<170^{\wedge} 2\right)\right)\right)$;

$\operatorname{img}\left(\left(\left(\left((\mathrm{xxx}-\mathrm{X}) \cdot{ }^{\wedge} 2+(\mathrm{yyy}-\mathrm{Y}) \cdot{ }^{\wedge} 2\right)>140^{\wedge} 2\right) \&\left(\left((\mathrm{xxx}-\mathrm{X}) \cdot{ }^{\wedge} 2+(\mathrm{yyy}-\right.\right.\right.\right.$

$\left.\left.\left.\left.\mathrm{Y}) .^{\wedge} 2\right)<170^{\wedge} 2\right)\right)\right)=1552 * \operatorname{uint} 16\left(\operatorname{img}\left(\left(\left((\mathrm{xxx}-\mathrm{X}) .^{\wedge} 2+(\mathrm{yyy}-\mathrm{Y}) .^{\wedge} 2\right)>140^{\wedge} 2\right) \&(((\mathrm{xxx}-\right.\right.$

$\left.\left.\left.\mathrm{X}) .^{\wedge} 2+(\mathrm{yyy}-\mathrm{Y}) .^{\wedge} 2\right)<170^{\wedge} 2\right)\right)>=$ lo_MLT_boneRi4 \& img $\left(\left(\left((\mathrm{xxx}-\mathrm{X}) .^{\wedge} 2+(\mathrm{yyy}-\right.\right.\right.$

$\left.\left.\left.\mathrm{Y}) .^{\wedge} 2\right)>140^{\wedge} 2\right) \&\left(\left((\mathrm{xxx}-\mathrm{X}) .^{\wedge} 2+(\mathrm{yyy}-\mathrm{Y}) \cdot{ }^{\wedge} 2\right)<170^{\wedge} 2\right)\right)$

$<=$ lo_MLT_hipRi4)+uint16(img $\left(\left(\left(((\mathrm{xxx}-\mathrm{X}))^{\wedge} 2+(\mathrm{yyy}-\mathrm{Y}) .^{\wedge} 2\right)>140^{\wedge} 2\right) \&(((\mathrm{xxx}-\right.$

$\left.\left.\left.\left.X) .^{\wedge} 2+(y y y-Y) . \wedge 2\right)<170^{\wedge} 2\right)\right)\right)<$ lo_MLT_boneRi4 I $\operatorname{img}\left(\left(\left(\left((x x x-X){ }^{\wedge} 2+(y y y-\right.\right.\right.\right.$

$\left.\left.\left.\mathrm{Y}) .^{\wedge} 2\right)>140^{\wedge} 2\right) \&\left(\left((\mathrm{xxx}-\mathrm{X}) .^{\wedge} 2+(\mathrm{yyy}-\mathrm{Y}) .^{\wedge} 2\right)<170^{\wedge} 2\right)\right)$

)$>$ lo_MLT_hipRi4).*img $\left(\left(\left((\mathrm{xxx}-\mathrm{X}) \cdot{ }^{\wedge} 2+(\mathrm{yyy}-\mathrm{Y}) .^{\wedge} 2\right)>140^{\wedge} 2\right) \&\left(\left((\mathrm{xxx}-\mathrm{X}) \cdot{ }^{\wedge} 2+(\mathrm{yyy}-\right.\right.\right.$

Y).^2)<170^2)) );

$\operatorname{img}\left(\left(\left(\left((\mathrm{xxx}-\mathrm{X}) \cdot{ }^{\wedge} 2+(\mathrm{yyy}-\mathrm{Y}) .^{\wedge} 2\right)>140^{\wedge} 2\right) \&\left(\left((\mathrm{xxx}-\mathrm{X}) \cdot{ }^{\wedge} 2+(\mathrm{yyy}-\right.\right.\right.\right.$

$\left.\left.\left.\left.\mathrm{Y}) .^{\wedge} 2\right)<170^{\wedge} 2\right)\right)\right)=1024^{*} \operatorname{uint} 16\left(\mathrm{img}\left(\left(\left((\mathrm{xxx}-\mathrm{X}) .^{\wedge} 2+(\mathrm{yyy}-\mathrm{Y}) .^{\wedge} 2\right)>140^{\wedge} 2\right) \&(((\mathrm{xxx}-\right.\right.$

$\left.\left.\left.\mathrm{X}) .^{\wedge} 2+(\mathrm{yyy}-\mathrm{Y}) .^{\wedge} 2\right)<170^{\wedge} 2\right)\right)>=\mathrm{lo} \_M L T \_h 20 R i 4 \& \operatorname{img}\left(\left(\left((\mathrm{xxx}-\mathrm{X}) .^{\wedge} 2+(\mathrm{yyy}-\right.\right.\right.$ 
$\left.\left.\left.\mathrm{Y}) .^{\wedge} 2\right)>140^{\wedge} 2\right) \&\left(\left((\mathrm{xxx}-\mathrm{X}) .^{\wedge} 2+(\mathrm{yyy}-\mathrm{Y}) .^{\wedge} 2\right)<170^{\wedge} 2\right)\right)$

$<=$ lo_MLT_boneRi4)+uint16(img $\left(\left(\left(\left(\right.\right.\right.\right.$ xxx-X).^2+(yyy-Y).$\left.\left.^{\wedge} 2\right)>140^{\wedge} 2\right) \&(((x x x-$ $\left.\left.\left.\left.\mathrm{X}) .^{\wedge} 2+(\mathrm{yyy}-\mathrm{Y}) .^{\wedge} 2\right)<170^{\wedge} 2\right)\right)\right)<\mathrm{lo} \_M L T \_h 20 R i 4 \mathrm{I} \operatorname{img}\left(\left(\left(\left((\mathrm{xxx}-\mathrm{X}) .^{\wedge} 2+(\mathrm{yyy}-\right.\right.\right.\right.$ $\left.\left.\left.\mathrm{Y}) .^{\wedge} 2\right)>140^{\wedge} 2\right) \&\left(\left((\mathrm{xxx}-\mathrm{X}) .^{\wedge} 2+(\mathrm{yyy}-\mathrm{Y}) .^{\wedge} 2\right)<170^{\wedge} 2\right)\right)$ )$>$ lo_MLT_boneRi4).*img $\left(\left(\left((\mathrm{xxx}-\mathrm{X}) \cdot^{\wedge} 2+(\mathrm{yyy}-\mathrm{Y}) .^{\wedge} 2\right)>140^{\wedge} 2\right) \&\left(\left((\mathrm{xxx}-\mathrm{X}) .^{\wedge} 2+(\mathrm{yyy}-\right.\right.\right.$ Y).^2)<170^2)) ); $\operatorname{img}\left(\left(\left(\left((\mathrm{xxx}-\mathrm{X}) .^{\wedge} 2+(\mathrm{yyy}-\mathrm{Y}) .^{\wedge} 2\right)>140^{\wedge} 2\right) \&\left(\left((\mathrm{xxx}-\mathrm{X}) \cdot{ }^{\wedge} 2+(\mathrm{yyy}-\right.\right.\right.\right.$ $\left.\left.\left.\left.\mathrm{Y}) .^{\wedge} 2\right)<170^{\wedge} 2\right)\right)\right)=928^{*} \operatorname{uint} 16\left(\operatorname{img}\left(\left(\left((\mathrm{xxx}-\mathrm{X}) .^{\wedge} 2+(\mathrm{yyy}-\mathrm{Y}) .^{\wedge} 2\right)>140^{\wedge} 2\right) \&(((\mathrm{xxx}-\right.\right.$ $\left.\left.\left.\mathrm{X}) .^{\wedge} 2+(\mathrm{yyy}-\mathrm{Y}) . \wedge 2\right)<170^{\wedge} 2\right)\right)>=\mathrm{lo} \_M L T \_a d i p o s e R i 4 \& \operatorname{img}\left(\left((\mathrm{xxx}-\mathrm{X}) .{ }^{\wedge} 2+(\mathrm{yyy}-\right.\right.$ $\left.\left.\left.\mathrm{Y}) .^{\wedge} 2\right)>140^{\wedge} 2\right) \&\left(\left((\mathrm{xxx}-\mathrm{X}) .^{\wedge} 2+(\mathrm{yyy}-\mathrm{Y}) \cdot{ }^{\wedge} 2\right)<170^{\wedge} 2\right)\right)$

$<=$ lo_MLT_h2oRi4)+uint16(img $\left(\left(\left(\left((x x x-X) .^{\wedge} 2+(y y y-Y) \cdot{ }^{\wedge} 2\right)>140^{\wedge} 2\right) \&(((x x x-\right.\right.$ $\left.\left.\left.\left.\mathrm{X}) .^{\wedge} 2+(\mathrm{yyy}-\mathrm{Y}) .^{\wedge} 2\right)<170^{\wedge} 2\right)\right)\right)<\mathrm{lo} \_M L T \_a d i p o s e R i 4 \mathrm{I} \operatorname{img}((((\mathrm{xxx}-\mathrm{X}) . \wedge 2+(\mathrm{yyy}-$ $\left.\left.\left.\mathrm{Y}) .^{\wedge} 2\right)>140^{\wedge} 2\right) \&\left(\left((\mathrm{xxx}-\mathrm{X}) \cdot^{\wedge} 2+(\mathrm{yyy}-\mathrm{Y}) \cdot{ }^{\wedge} 2\right)<170^{\wedge} 2\right)\right)$

)$>$ lo_MLT_h2oRi4).*img $\left(\left(\left((\mathrm{xxx}-\mathrm{X}) .^{\wedge} 2+(\mathrm{yyy}-\mathrm{Y}) .^{\wedge} 2\right)>140^{\wedge} 2\right) \&\left(\left((\mathrm{xxx}-\mathrm{X}) .^{\wedge} 2+(\mathrm{yyy}-\right.\right.\right.$ Y).^2)<170^2)) );

$\% * * * * * * * * * * * * * * * * * * * * * * * * * * * * * \% \mathrm{Ring} 5 \% * * * * * * * * * * * * * * * * * * * * * * * * * * * * \%$ $\%$ Converting pixel values into HUs using the threshold values defined above for each material in this ring

$\operatorname{img}\left(\left(\left((\mathrm{xxx}-\mathrm{X}) \cdot{ }^{\wedge} 2+(\mathrm{yyy}-\mathrm{Y}) \cdot{ }^{\wedge} 2\right)>170^{\wedge} 2\right) \&\left(\left((\mathrm{xxx}-\mathrm{X}) \cdot{ }^{\wedge} 2+(\mathrm{yyy}-\right.\right.\right.$

$\left.\left.\left.\left.\mathrm{Y}) .^{\wedge} 2\right)<231^{\wedge} 2\right)\right)\right)=1024 * \operatorname{uint} 16\left(\operatorname{img}\left(\left((\mathrm{xxx}-\mathrm{X}) .^{\wedge} 2+(\mathrm{yyy}-\mathrm{Y}) \cdot{ }^{\wedge} 2\right)>170^{\wedge} 2\right) \&(((\mathrm{xxx}-\right.$ $\left.\left.\left.\mathrm{X}) .{ }^{\wedge} 2+(\mathrm{yyy}-\mathrm{Y}) . \wedge 2\right)<231^{\wedge} 2\right)\right)>=\mathrm{lo} \_M L T \_h 20 R i 5 \& \operatorname{img}(((\mathrm{xxx}-\mathrm{X}) . \wedge 2+(\mathrm{yyy}-$ $\left.\left.\left.\left.\mathrm{Y}) .^{\wedge} 2\right)>170^{\wedge} 2\right) \&\left(\left((\mathrm{xxx}-\mathrm{X}) .^{\wedge} 2+(\mathrm{yyy}-\mathrm{Y}) .^{\wedge} 2\right)<231^{\wedge} 2\right)\right)<=3000\right)+\mathrm{uint} 16(\mathrm{img}(((((\mathrm{xxx}-$ $\left.\left.\left.\left.\mathrm{X}) .^{\wedge} 2+(\mathrm{yyy}-\mathrm{Y}) \cdot{ }^{\wedge} 2\right)>170^{\wedge} 2\right) \&\left(\left((\mathrm{xxx}-\mathrm{X}) .^{\wedge} 2+(\mathrm{yyy}-\mathrm{Y}) . \wedge 2\right)<231^{\wedge} 2\right)\right)\right)<$ lo_MLT_h2oRi5 I $\operatorname{img}\left(\left(\left(\left((\mathrm{xxx}-\mathrm{X}) .^{\wedge} 2+(\mathrm{yyy}-\mathrm{Y}) \cdot{ }^{\wedge} 2\right)>170^{\wedge} 2\right) \&\left(\left((\mathrm{xxx}-\mathrm{X}) .^{\wedge} 2+(\mathrm{yyy}-\mathrm{Y}) .^{\wedge} 2\right)<231^{\wedge} 2\right)\right)\right.$ )$>3000) \cdot * \operatorname{img}\left(\left(\left(\left((x x x-X) .^{\wedge} 2+(y y y-Y) .^{\wedge} 2\right)>170^{\wedge} 2\right) \&\left(\left((x x x-X){ }^{\wedge} 2+(y y y-\right.\right.\right.\right.$ $\left.\left.\left.\left.Y) .^{\wedge} 2\right)<231^{\wedge} 2\right)\right)\right)$;

$\operatorname{img}\left(\left(\left((\mathrm{xxx}-\mathrm{X}) \cdot^{\wedge} 2+(\mathrm{yyy}-\mathrm{Y}) \cdot^{\wedge} 2\right)>170^{\wedge} 2\right) \&\left(\left((\mathrm{xxx}-\mathrm{X}) \cdot{ }^{\wedge} 2+(\mathrm{yyy}-\right.\right.\right.$ $\left.\left.\left.\left.\mathrm{Y}) .^{\wedge} 2\right)<231^{\wedge} 2\right)\right)\right)=928^{*} \operatorname{uint} 16\left(\operatorname{img}\left(\left(\left((\mathrm{xxx}-\mathrm{X}) .^{\wedge} 2+(\mathrm{yyy}-\mathrm{Y}) .^{\wedge} 2\right)>170^{\wedge} 2\right) \&(((\mathrm{xxx}-\right.\right.$ $\left.\left.\left.\mathrm{X}) .^{\wedge} 2+(\mathrm{yyy}-\mathrm{Y}) .^{\wedge} 2\right)<231^{\wedge} 2\right)\right)>=\mathrm{lo} \_M L T \_a d i p o s e R i 5 \& \operatorname{img}\left(\left((\mathrm{xxx}-\mathrm{X}) .^{\wedge} 2+(\mathrm{yyy}-\right.\right.$ $\left.\left.\left.\mathrm{Y}) .^{\wedge} 2\right)>170^{\wedge} 2\right) \&\left(\left((\mathrm{xxx}-\mathrm{X}) .^{\wedge} 2+(\mathrm{yyy}-\mathrm{Y}) .^{\wedge} 2\right)<231^{\wedge} 2\right)\right)$

$<=$ lo_MLT_h2oRi5)+uint16(img $\left(\left(\left(\left(\right.\right.\right.\right.$ xxx-X).^2+(yyy-Y). $\left.\left.{ }^{\wedge} 2\right)>170^{\wedge} 2\right) \&(((x x x-$ X).^2+(yyy-Y).^2)<231^2)) $)<$ lo_MLT_adiposeRi5 I img $\left(\left(\left(\left(\right.\right.\right.\right.$ xxx-X) $.^{\wedge} 2+($ yyy$\left.\left.\left.\mathrm{Y}) .^{\wedge} 2\right)>170^{\wedge} 2\right) \&\left(\left((\mathrm{xxx}-\mathrm{X}) \cdot{ }^{\wedge} 2+(\mathrm{yyy}-\mathrm{Y}) \cdot{ }^{\wedge} 2\right)<231^{\wedge} 2\right)\right)$

)$>$ lo_MLT_h2oRi5).*img $\left(\left(\left((\mathrm{xxx}-\mathrm{X}) .^{\wedge} 2+(\mathrm{yyy}-\mathrm{Y}) .^{\wedge} 2\right)>170^{\wedge} 2\right) \&\left(\left((\mathrm{xxx}-\mathrm{X}) .^{\wedge} 2+(\mathrm{yyy}-\right.\right.\right.$ Y).^2)<231^2)) );

$\operatorname{img}\left(\left(\left(\left((x x x-X) \cdot{ }^{\wedge} 2+(y y y-Y) \cdot{ }^{\wedge} 2\right)>170^{\wedge} 2\right) \&\left(\left((x x x-X) \cdot{ }^{\wedge} 2+(y y y-\right.\right.\right.\right.$ $\left.\left.\left.\left.\mathrm{Y}) .^{\wedge} 2\right)<231^{\wedge} 2\right)\right)\right)=48^{*} \operatorname{uint} 16\left(\operatorname{img}\left(\left(\left((\mathrm{xxx}-\mathrm{X}) .^{\wedge} 2+(\mathrm{yyy}-\mathrm{Y}) .^{\wedge} 2\right)>170^{\wedge} 2\right) \&(((\mathrm{xxx}-\right.\right.$ $\left.\left.\left.\mathrm{X}) .^{\wedge} 2+(\mathrm{yyy}-\mathrm{Y}) .^{\wedge} 2\right)<231^{\wedge} 2\right)\right)>=49 \& \operatorname{img}\left(\left(\left((\mathrm{xxx}-\mathrm{X}) .^{\wedge} 2+(\mathrm{yyy}-\mathrm{Y}) .^{\wedge} 2\right)>170^{\wedge} 2\right) \&(((\mathrm{xxx}-\right.$ $\left.\left.\left.\mathrm{X}) .^{\wedge} 2+(\mathrm{yyy}-\mathrm{Y}) .^{\wedge} 2\right)<231^{\wedge} 2\right)\right)<=$ lo_MLT_adiposeRi5)+uint16(img $(((((\mathrm{xxx}-$ $\left.\left.\left.\left.\mathrm{X}) .^{\wedge} 2+(\mathrm{yyy}-\mathrm{Y}) \cdot{ }^{\wedge} 2\right)>170^{\wedge} 2\right) \&\left(\left((\mathrm{xxx}-\mathrm{X}) .^{\wedge} 2+(\mathrm{yyy}-\mathrm{Y}) .^{\wedge} 2\right)<231^{\wedge} 2\right)\right)\right)<49 \mid \operatorname{img}(((((\mathrm{xxx}-$ $\left.\left.\left.\mathrm{X}) .^{\wedge} 2+(\mathrm{yyy}-\mathrm{Y}) .^{\wedge} 2\right)>170^{\wedge} 2\right) \&\left(\left((\mathrm{xxx}-\mathrm{X}) .^{\wedge} 2+(\mathrm{yyy}-\mathrm{Y}) .^{\wedge} 2\right)<231^{\wedge} 2\right)\right)$ )$>$ lo_MLT_adiposeRi5).*img $\left(\left(\left(\left(\right.\right.\right.\right.$ xxx-X).^2+(yyy-Y).$\left.\left.^{\wedge} 2\right)>170^{\wedge} 2\right) \&(((x x x-$ 
X).^2+(ууу-Y).^2)<231^2)) );

$\%$ correcting low $\mathrm{HU}$ (air) in high density region (metals)

$\operatorname{img}((\mathrm{X}-50: \mathrm{X}+50),(\mathrm{Y}-180: \mathrm{Y}-58))=928 * \operatorname{uint} 16(\mathrm{img}((\mathrm{X}-50: \mathrm{X}+50),(\mathrm{Y}-180: \mathrm{Y}-$ 58) $)>=0 \& \operatorname{img}((\mathrm{X}-50: \mathrm{X}+50),(\mathrm{Y}-180: \mathrm{Y}-58))<=927)+u i n t 16(\operatorname{img}((\mathrm{X}-50: \mathrm{X}+50),(\mathrm{Y}-$ 180:Y-58) $)<0$ I $\operatorname{img}((\mathrm{X}-50: \mathrm{X}+50),(\mathrm{Y}-180: \mathrm{Y}-58))>927) . \operatorname{img}((\mathrm{X}-50: \mathrm{X}+50),(\mathrm{Y}-180: \mathrm{Y}-$ $58)$;

$\operatorname{img}((\mathrm{X}-50: \mathrm{X}+50),(\mathrm{Y}+49: \mathrm{Y}+182))=928 * \operatorname{uint} 16(\mathrm{img}((\mathrm{X}-$

$50: \mathrm{X}+50),(\mathrm{Y}+49: \mathrm{Y}+182))>=0 \& \operatorname{img}((\mathrm{X}-$

$50: \mathrm{X}+50),(\mathrm{Y}+49: \mathrm{Y}+182))<=927)+u i n t 16(\mathrm{img}((\mathrm{X}-50: \mathrm{X}+50),(\mathrm{Y}+49: \mathrm{Y}+182))<0$ ।

$\operatorname{img}((\mathrm{X}-50: \mathrm{X}+50),(\mathrm{Y}+49: \mathrm{Y}+182))>927) . * \operatorname{img}((\mathrm{X}-50: \mathrm{X}+50),(\mathrm{Y}+49: \mathrm{Y}+182))$;

$\%$ to exculde coach

$\operatorname{img}($ coach:410,1:410 $)=48 *$ uint $16(\operatorname{img}(\operatorname{coach}: 410,1: 410)>=0$ \&

$\operatorname{img}(\operatorname{coach}: 410,1: 410)<=9000)+\operatorname{uint} 16(\operatorname{img}(\operatorname{coach}: 410,1: 410)<0$ ।

$\operatorname{img}($ coach:410,1:410)>9000).*img(coach:410,1:410);

$\%$ to exclude the high HU in the top

$\operatorname{img}(1: 110,1: 410)=1024 * \operatorname{uint} 16(\operatorname{img}(1: 110,1: 410)>=1025 \&$

$\operatorname{img}(1: 110,1: 410)<=9000)+$ uint $16(\operatorname{img}(1: 110,1: 410)<1025$ ।

$\operatorname{img}(1: 110,1: 410)>9000) .{ }^{*} \operatorname{img}(1: 110,1: 410)$;

dicomwrite(img, ['Bi' num2str(i) '.dcm'], imgHdr,'CreateMode','Copy')

end

close all 


\section{Reference}

AARUP, L. R., NAHUM, A. E., ZACHARATOU, C., JUHLER-NØTTRUP, T., KNÖÖS, T., NYSTRÖM, H., SPECHT, L., WIESLANDER, E. \& KORREMAN, S. S. 2009. The effect of different lung densities on the accuracy of various radiotherapy dose calculation methods: implications for tumour coverage. Radiotherapy and Oncology, 91, 405-414.

AHNESJÖ, A. 1989. Collapsed cone convolution of radiant energy for photon dose calculation in heterogeneous media. Medical physics, 16, 577-592.

AHNESJÖ, A. \& ASPRADAKIS, M. M. 1999. Dose calculations for external photon beams in radiotherapy. Physics in medicine and biology, 44, R99.

AHNESJÖ, A., SAXNER, M. \& TREPP, A. 1992. A pencil beam model for photon dose calculation. Medical physics, 19, 263-273.

AHNESJÖ, A., WEBER, L., MURMAN, A., SAXNER, M., THORSLUND, I. \& TRANEUS, E. 2005. Beam modeling and verification of a photon beam multisource model. Medical physics, 32, 1722-1737.

AIRD, E. G. A. \& CONWAY, J. 2002 . CT simulation for radiotherapy treatment planning. The British journal of radiology, 75, 937-949.

ALMATANI, T. 2011. Treatment planning using CBCT. Master Thesis, Swansea University.

ALMATANI, T., HUGTENBURG, R. P., LEWIS, R., BARLEY, S. \& EDWARDS, M. 2016a. Simplified material assignment for cone beam computed tomography-based dose calculations of prostate radiotherapy with hip prostheses. Journal of Radiotherapy in Practice, 15, 170-180.

ALMATANI, T., HUGTENBURG, R. P., LEWIS, R. D., BARLEY, S. E. \& EDWARDS, M. A. 2016b. Automated algorithm for CBCT-based dose calculations of prostate radiotherapy with bilateral hip prostheses. The British Journal of Radiology, 89, 20160443.

ALMATANI, T., HUGTENBURG, R. P., LEWIS, R. D., BARLEY, S. E. \& EDWARDS, M. A. 2017. Dosimetric feasibility of magnetic resonance (MR)based dose calculation of prostate radiotherapy using multilevel threshold algorithm. Journal of Radiotherapy in Practice, 16, 415-422. 
ALMBERG, S. S., FRENGEN, J., KYLLING, A. \& LINDMO, T. 2012. Monte Carlo linear accelerator simulation of megavoltage photon beams: independent determination of initial beam parameters. Medical physics, 39, 40-47.

ALSALEH, W. 2014. The prediction of secondary cancer risk after high-energy Xray radiotherapy using the Monte Carlo method and voxel phantoms. $\mathrm{PhD}$ Thesis, Swansea University.

ALTUNBAS, C., LAI, C.-J., ZHONG, Y. \& SHAW, C. C. 2014. Reduction of ring artifacts in CBCT: Detection and correction of pixel gain variations in flat panel detectors. Medical physics, 41,091913.

ANAS, E. M. A., KIM, J. G., LEE, S. Y. \& HASAN, M. K. 2011. Comparison of ring artifact removal methods using flat panel detector based CT images.

Biomedical engineering online, 10, 72.

ANDREASEN, D., VAN LEEMPUT, K. \& EDMUND, J. M. 2016. A patch-based pseudo-CT approach for MRI-only radiotherapy in the pelvis. Medical Physics, 43, 4742-4752.

ANDREO, P. 1985. The interaction of electrons with matter II. scattering. The Computation of Dose Distributions in Electron Beam Radiotherapy. Umea University Sweden.

ANDREO, P. 1991. Monte Carlo techniques in medical radiation physics. Physics in medicine and biology, 36, 861 .

ANTONUK, L. E., YORKSTON, J., HUANG, W., SANDLER, H., SIEWERDSEN, J. H. \& EL-MOHRI, Y. 1996. Megavoltage imaging with a large-area, flatpanel, amorphous silicon imager. International Journal of Radiation Oncology* Biology* Physics, 36, 661-672.

ATTIX, F. H. 2008. Introduction to radiological physics and radiation dosimetry, John Wiley \& Sons.

BANSAL, A., KAPOOR, R., SINGH, S. K., KUMAR, N., OINAM, A. S. \& SHARMA, S. C. 2012. Dosimetric comparison of standard three-dimensional conformal radiotherapy followed by intensity-modulated radiotherapy boost schedule (sequential IMRT plan) with simultaneous integrated boost-IMRT (SIB IMRT) treatment plan in patients with localized carcinoma prostate. Indian journal of urology: IJU: journal of the Urological Society of India, 28, 300. 
BARO, J., SEMPAU, J., FERNÁNDEZ-VAREA, J. M. \& SALVAT, F. 1995. PENELOPE: an algorithm for Monte Carlo simulation of the penetration and energy loss of electrons and positrons in matter. Nuclear Instruments and Methods in Physics Research Section B: Beam Interactions with Materials and Atoms, 100, 31-46.

BARRETT, A., DOBBS, J. \& ROQUES, T. 2009. Practical Radiotherapy Planning Fourth Edition, CRC Press.

BATHO, H. F. 1964. Lung corrections in cobalt 60 beam therapy. Journal of the Canadian Association of Radiologists, 15, 79.

BENTZEN, S. M. 2005. Theragnostic imaging for radiation oncology: dose-painting by numbers. The lancet oncology, 6, 112-117.

BERGER, M. J. 1963. Monte Carlo calculation of the penetration and diffusion of fast charged particles. Methods in computational physics, 1, 135-215.

BERGER, M. J. \& HUBBELL, J. H. XCOM, Photon cross sections on a personal computer. Report No. NBSIR87-3597. Gaithersburg, MD: NIST; 1987.

BERGER, M. J. \& SELTZER, S. M. 1973. ETRAN Monte Carlo code system for electron and photon transport through extended media. Radiation Shielding Information Center (RSIC) Report CCC-107, Oak Ridge National Laboratory, Oak Ridge, TN, 75.

BHABHA, H. J. 1936. The scattering of positrons by electrons with exchange on Dirac's theory of the positron. Proceedings of the Royal Society of London Series A: Mathematical, Physical \& Engineering Sciences, 154, 195-206.

BIELAJEW, A. F., ROGERS, D. W. O., JENKINS, T. W., NELSON, W. R. \& RINDI, A. 1988. Monte Carlo Transport of electrons and photons. et al. Plenum Press, New York.

BODA-HEGGEMANN, J., LOHR, F., WENZ, F., FLENTJE, M. \& GUCKENBERGER, M. 2011. kV cone-beam CT-based IGRT. Strahlentherapie und Onkologie, 187, 284-291.

BOGGULA, R., LORENZ, F., ABO-MADYAN, Y., LOHR, F., WOLFF, D., BODAHEGGEMANN, J., HESSER, J., WENZ, F. \& WERTZ, H. 2009. A new strategy for online adaptive prostate radiotherapy based on cone-beam CT. Zeitschrift für Medizinische Physik, 19, 264-276. 
BOGGULA, R., WERTZ, H., LORENZ, F., MADYAN, Y. A., BODA-

HEGGEMANN, J., SCHNEIDER, F., POLEDNIK, M., HESSER, J., LOHR, F. \& WENZ, F. 2007. A proposed strategy to implement CBCT images for replanning and dose calculations. International Journal of Radiation Oncology* Biology* Physics, 69, S655-S656.

BOL, G. H., HISSOINY, S., LAGENDIJK, J. J. W. \& RAAYMAKERS, B. W. 2012. Fast online Monte Carlo-based IMRT planning for the MRI linear accelerator. Physics in medicine and biology, 57, 1375.

BOONE, J. M. \& SEIBERT, J. A. 1988. An analytical model of the scattered radiation distribution in diagnostic radiology. Medical physics, 15, 721-725. BOURLAND, J. D. 2012. Image-guided Radiation Therapy, Crc Press. BOYDEV, C., TALEB-AHMED, A., DERRAZ, F., PEYRODIE, L., THIRAN, J.-P. \& PASQUIER, D. 2015. Development of CBCT-based prostate setup correction strategies and impact of rectal distension. Radiation Oncology, 10, 1.

BRIESMEISTER, J. F. 1986. MCNP-A general Monte Carlo code for neutron and photon transport. $L a-7396-m$.

BRUN, R., HAGELBERG, R., HANSROUL, M. \& LASSALLE, J. C. 1978. GEANT: Simulation program for particle physics experiments. User guide and reference manual. CERN Rep. CERN-DD-78-2.

BURRIDGE, N., AMER, A., MARCHANT, T., SYKES, J., STRATFORD, J., HENRY, A., MCBAIN, C., PRICE, P. \& MOORE, C. 2006. Online adaptive radiotherapy of the bladder: small bowel irradiated-volume reduction. International Journal of Radiation Oncology* Biology* Physics, 66, 892-897.

CANCER RESEARCH UK. 2003. Incidence, 2003 [Online]. Cancer Research UK.

Available: http://www.cancerresearchuk.org/cancerinfo/cancerstats/types/prostate/incidence/ [Accessed November 2013].

CANCER RESEARCH UK. 2004. Mortality, 2004 [Online]. Cancer Research UK. Available: http://www.cancerresearchuk.org/cancerinfo/cancerstats/types/prostate/mortality/ [Accessed November 2013].

CANCER RESEARCH UK. 2014. Cancer Research UK. Available: http://www.cancerresearchuk.org/health-professional/cancer- 
$\underline{\text { statistics/statistics-by-cancer-type/prostate-cancer - heading-One [Accessed }}$ August 2016].

CANCER RESEARCH UK PACE TRIAL. 2016. Cancer Research UK Available: http://www.cancerresearchuk.org/about-cancer/find-a-clinical-trial/a-trialcomparing-surgery-conventional-radiotherapy-and-stereotactic-radiotherapyfor-localised-prostate-cancer-pace - undefined [Accessed December 2016].

CARRASCO, P., JORNET, N., DUCH, M. A., WEBER, L., GINJAUME, M., EUDALDO, T., JURADO, D., RUIZ, A. \& RIBAS, M. 2004. Comparison of dose calculation algorithms in phantoms with lung equivalent heterogeneities under conditions of lateral electronic disequilibrium. Medical physics, 31, 2899-2911.

CEDRIC, X. Y.\& WONG, J. W. 1993. Implementation of the ETAR method for 3D inhomogeneity correction using FFT. Medical physics, 20, 627-632.

CHAI, X., VAN HERK, M., BETGEN, A., HULSHOF, M. \& BEL, A. 2012. Automatic bladder segmentation on CBCT for multiple plan ART of bladder cancer using a patient-specific bladder model. Physics in medicine and biology, 57, 3945 .

CHAN, M. F., YANG, J., SONG, Y., BURMAN, C., CHAN, P. \& LI, S. 2011. Evaluation of imaging performance of major image guidance systems. Biomedical imaging and intervention journal, 7.

CHEN, G. T. Y. 1988. Dose volume histograms in treatment planning. International Journal of Radiation Oncology* Biology* Physics, 14, 1319-1320.

CHEUNG, J., AUBRY, J.-F., YOM, S. S., GOTTSCHALK, A. R., CELI, J. C. \& POULIOT, J. 2009. Dose recalculation and the dose-guided radiation therapy (DGRT) process using megavoltage cone-beam CT. International Journal of Radiation Oncology* Biology* Physics, 74, 583-592.

CHIBANI, O. \& LI, X. A. 2002. Monte Carlo dose calculations in homogeneous media and at interfaces: a comparison between GEPTS, EGSnrc, MCNP, and measurements. Medical physics, 29, 835-847.

CIERNIK, I. F., BAUMERT, B. G., EGLI, P., GLANZMANN, C. \& LÜTOLF, U. M. 2002. On-line correction of beam portals in the treatment of prostate cancer using an endorectal balloon device. Radiotherapy and oncology, 65, 39-45. 
CULLEN, D. E., PERKINS, S. T. \& RATHKOPF, J. A. 1990. The 1989 Livermore evaluated photon data library (EPDL). Lawrence Livermore National Lab., CA (USA).

D’AVINO, V., PALMA, G., LIUZZI, R., CONSON, M., DORIA, F., SALVATORE, M., PACELLI, R. \& CELLA, L. 2015. Prediction of gastrointestinal toxicity after external beam radiotherapy for localized prostate cancer. Radiation Oncology, 10, 1.

DAVIS, A., PEARSON, E., PAN, X. \& PELIZZARI, C. 2014. WE-G-BRF-07: NonCircular Scanning Trajectories with Varian Developer Mode. Medical Physics, 41, 523-523.

DAWSON, L. A. \& JAFFRAY, D. A. 2007. Advances in image-guided radiation therapy. Journal of clinical oncology, 25, 938-946.

DE CREVOISIER, R., TUCKER, S. L., DONG, L., MOHAN, R., CHEUNG, R., COX, J. D. \& KUBAN, D. A. 2005. Increased risk of biochemical and local failure in patients with distended rectum on the planning CT for prostate cancer radiotherapy. International Journal of Radiation Oncology* Biology* Physics, 62, 965-973.

DE MAN, B. \& BASU, S. 2004. Distance-driven projection and backprojection in three dimensions. Physics in medicine and biology, 49, 2463.

DE MEERLEER, G. O., VAKAET, L. A. M. L., DE GERSEM, W. R. T., DE WAGTER, C., DE NAEYER, B. \& DE NEVE, W. 2000. Radiotherapy of prostate cancer with or without intensity modulated beams: a planning comparison. International Journal of Radiation Oncology* Biology* Physics, 47, 639-648.

DEARNALEY, D., SYNDIKUS, I., MOSSOP, H., BIRTLE, A., BLOOMFIELD, D., CRUICKSHANK, C., GRAHAM, J., HASSAN, S., KHOO, V . \& LOGUE, J. P. 2015. 5 year outcomes of a phase III randomised trial of conventional or hypofractionated high dose intensity modulated radiotherapy for prostate cancer (CRUK/06/016): report from the CHHiP Trial Investigators Group. European Journal of Cancer.

DEASY, J. O., BLANCO, A. I. \& CLARK, V. H. 2003. CERR: a computational environment for radiotherapy research. Medical physics, 30, 979-985. 
DEVIC, S. 2012. MRI simulation for radiotherapy treatment planning. Medical physics, 39, 6701-6711.

DING, G. X., CYGLER, J. E., YU, C. W., KALACH, N. I. \& DASKALOV, G. 2005. A comparison of electron beam dose calculation accuracy between treatment planning systems using either a pencil beam or a Monte Carlo algorithm. International Journal of Radiation Oncology* Biology* Physics, 63, 622-633. DOEMER, A., CHETTY, I. J., GLIDE-HURST, C., NURUSHEV, T., HEARSHEN, D., PANTELIC, M., TRAUGHBER, M., KIM, J., LEVIN, K. \& ELSHAIKH, M. A. 2015. Evaluating organ delineation, dose calculation and daily localization in an open-MRI simulation workflow for prostate cancer patients. Radiation Oncology, 10, 1.

DOWLING, J. A., LAMBERT, J., PARKER, J., SALVADO, O., FRIPP, J., CAPP, A., WRATTEN, C., DENHAM, J. W. \& GREER, P. B. 2012. An atlas-based electron density mapping method for magnetic resonance imaging (MRI)alone treatment planning and adaptive MRI-based prostate radiation therapy. International Journal of Radiation Oncology* Biology* Physics, 83, e5-e11. DOWNES, P., YAIKHOM, G., GIDDY, J. P., WALKER, D. W., SPEZI, E. \& LEWIS, D. G. 2009. High-performance computing for Monte Carlo radiotherapy calculations. Philosophical Transactions of the Royal Society A: Mathematical, Physical and Engineering Sciences, 367, 2607-2617.

DUDERSTADT, J. J. \& MARTIN, W. R. 1979. Transport Theory. Wiley, New York. DUNLOP, A., MCQUAID, D., NILL, S., MURRAY, J., POLUDNIOWSKI, G., HANSEN, V . N., BHIDE, S., NUTTING, C., HARRINGTON, K. \& NEWBOLD, K. 2015. Comparison of CT number calibration techniques for CBCT-based dose calculation. Strahlentherapie und Onkologie, 191, 970-978. EDMUND, J. M., ANDREASEN, D., MAHMOOD, F. \& VAN LEEMPUT, K. 2015. Cone beam computed tomography guided treatment delivery and planning verification for magnetic resonance imaging only radiotherapy of the brain. Acta Oncologica, 54, 1496-1500.

EILERTSEN, K., NILSEN TOR ARNE VESTAD, L., GEIER, O. \& SKRETTING, A. 2008. A simulation of MRI based dose calculations on the basis of radiotherapy planning CT images. Acta Oncologica, 47, 1294-1302. 
FADDEGON, B. A., KAWRAKOW, I., KUBYSHIN, Y., PERL, J., SEMPAU, J. \& URBAN, L. 2009. The accuracy of EGSnrc, Geant4 and PENELOPE Monte Carlo systems for the simulation of electron scatter in external beam radiotherapy. Physics in medicine and biology, 54, 6151.

FAN, Q., LU, B., PARK, J. C., NIU, T., LI, J. G., LIU, C. \& ZHU, L. 2015. Imagedomain shading correction for cone-beam CT without prior patient information. Journal of Applied Clinical Medical Physics, 16.

FELDKAMP, L. A., DAVIS, L. C. \& KRESS, J. W. 1984. Practical cone-beam algorithm. Josa a, 1, 612-619.

FELLER, V. 1950. An Introduction to Probability Theory and Its Applications: Volume One, John Wiley \& Sons.

FEUVRET, L., NOËL, G., MAZERON, J.-J. \& BEY, P. 2006. Conformity index: a review. International Journal of Radiation Oncology* Biology* Physics, 64, 333-342.

FIORINO, C., VALDAGNI, R., RANCATI, T. \& SANGUINETI, G. 2009. Dosevolume effects for normal tissues in external radiotherapy: pelvis. Radiotherapy and Oncology, 93, 153-167.

FIPPEL, M. 1999. Fast Monte Carlo dose calculation for photon beams based on the VMC electron algorithm. Medical physics, 26, 1466-1475.

FOTINA, I., HOPFGARTNER, J., STOCK, M., STEININGER, T., LÜTGENDORFCAUCIG, C. \& GEORG, D. 2012. Feasibility of CBCT-based dose calculation: comparative analysis of HU adjustment techniques. Radiotherapy and Oncology, 104, 249-256.

FRANCESCON, P., CORA, S. \& CHIOVATI, P. 2003. Dose verification of an IMRT treatment planning system with the BEAM EGS4-based Monte Carlo code. Medical physics, 30, 144-157.

FÜTTERER, J. J., BARENTSZ, J. O. \& HEIJMINK, S. W. 2008. Value of 3-T magnetic resonance imaging in local staging of prostate cancer. Topics in Magnetic Resonance Imaging, 19, 285-289.

GILES, D. M. 2010. Cone-Beam Computed Tomography: Imaging Dose during CBCT Scan Acquisition and Accuracy of CBCT Based Dose Calculations.

GLATSTEIN, E. Intensity-modulated radiation therapy: the inverse, the converse, and the perverse. Seminars in radiation oncology, 2002. Elsevier, 272-281. 
GRAHAM, S. A., MOSELEY, D. J., SIEWERDSEN, J. H. \& JAFFRAY, D. A. 2007. Compensators for dose and scatter management in cone-beam computed tomography. Medical physics, 34, 2691-2703.

GREEN, E. M., FORREST, L. J. \& ADAMS, W. M. 2002. A vacuum-formable mattress for veterinary radiotherapy positioning: comparison with conventional methods. Veterinary radiology \& ultrasound: the official journal of the American College of Veterinary Radiology and the International Veterinary Radiology Association, 44, 476-479.

GUAN, H. \& DONG, H. 2009. Dose calculation accuracy using cone-beam CT (CBCT) for pelvic adaptive radiotherapy. Physics in medicine and biology, 54, 6239 .

GUTIERREZ, S., DESCAMPS, B. \& VANHOVE, C. 2015. MRI-Only Based Radiotherapy Treatment Planning for the Rat Brain on a Small Animal Radiation Research Platform (SARRP). PloS one, 10, e0143821.

HANSEN, E. K., BUCCI, M. K., QUIVEY, J. M., WEINBERG, V. \& XIA, P. 2006. Repeat CT imaging and replanning during the course of IMRT for head-andneck cancer. International Journal of Radiation Oncology* Biology* Physics, 64, 355-362.

HATTON, J., MCCURDY, B. \& GREER, P. B. 2009. Cone beam computerized tomography: the effect of calibration of the Hounsfield unit number to electron density on dose calculation accuracy for adaptive radiation therapy. Physics in medicine and biology, 54, N329.

HELD, M., SNEED, P. K., FOGH, S. E., POULIOT, J. \& MORIN, O. 2015.

Feasibility of MV CBCT-based treatment planning for urgent radiation therapy: dosimetric accuracy of MV CBCT-based dose calculations. Journal of Applied Clinical Medical Physics, 16.

HILLE, A., TÖWS, N., SCHMIDBERGER, H. \& HESS, C. F. 2005. A Prospective Three-Dimensional Analysis about the Impact of Differences in the Clinical Target Volume in Prostate Cancer Irradiation on Normal-Tissue Exposure. Strahlentherapie und Onkologie, 181, 789-795.

HOSKIN, P. J. 2008. On target: ensuring geometric accuracy in radiotherapy, Royal College of Radiologists. 
HPC-WALES. Bangor, UK. Available: http://www.hpcwales.co.uk [Accessed December 2016.

HSIEH, J., MOLTHEN, R. C., DAWSON, C. A. \& JOHNSON, R. H. 2000. An iterative approach to the beam hardening correction in cone beam CT. Medical physics, 27, 23-29.

HSU, S.-H., CAO, Y., HUANG, K., FENG, M. \& BALTER, J. M. 2013.

Investigation of a method for generating synthetic CT models from MRI scans of the head and neck for radiation therapy. Physics in medicine and biology, $58,8419$.

HU, W., YE, J., WANG, J., MA, X. \& ZHANG, Z. 2010. Use of kilovoltage X-ray volume imaging in patient dose calculation for head-and-neck and partial brain radiation therapy. Radiation Oncology, 5, 1 .

HYER, D. E., SERAGO, C. F., KIM, S., LI, J. G. \& HINTENLANG, D. E. 2010. An organ and effective dose study of XVI and OBI cone-beam CT systems. Journal of Applied Clinical Medical Physics, 11.

ICRU 2010. Prescribing, recording, and reporting photon-beam intensity-modulated radiation therapy (IMRT). ICRU Report 83. J icru, 10, 1-106.

INTERNATIONAL ELECTROTECHNICAL, C. 1998. Medical electrical equipment-Part 2-1: Particular requirements for the safety of electron accelerators in the range of $1 \mathrm{MeV}$ to $50 \mathrm{MeV}$. Geneva: IEC.

ITALIA, C., FIORINO, C., CIOCCA, M., CATTANEO, G. M., MONTANARO, P., BOLOGNESI, A., LANCENI, A., RENI, M., BONINI, A. \& MODUGNO, A. 1997. Quality control by portal film analysis in radiotherapy for prostate cancer: a comparison between two different institutions and treatment techniques. Tumori, 84, 640-648.

JACKSON JOHN, D. 1999. Classical electrodynamics. John Wiley \& Sons, Inc., New York, NY.

JAFFRAY, D. A. \& SIEWERDSEN, J. H. 2000. Cone-beam computed tomography with a flat-panel imager: initial performance characterization. Medical physics, 27, 1311-1323.

JAFFRAY, D. A., SIEWERDSEN, J. H., WONG, J. W. \& MARTINEZ, A. A. 2002. Flat-panel cone-beam computed tomography for image-guided radiation 
therapy. International Journal of Radiation Oncology* Biology* Physics, 53, 1337-1349.

JARRY, G., GRAHAM, S. A., JAFFRAY, D. A., MOSELEY, D. J. \&

VERHAEGEN, F. Scatter correction for kilovoltage cone-beam computed tomography $(\mathrm{CBCT})$ images using Monte Carlo simulations. Medical Imaging, 2006. International Society for Optics and Photonics, 614254614254-10.

JOHANSSON, A., KARLSSON, M. \& NYHOLM, T. 2011. CT substitute derived from MRI sequences with ultrashort echo time. Medical physics, 38, 27082714.

JOHNS, H. E. 1983. Physics of radiology, Charles River Media.

JOHNS, H. E. \& CUNNINGHAM, J. R. 1983. The Physics of Radiology 4th edn (Springfield, IL: Thomas).

KAMATH, S., SONG, W., CHVETSOV, A., OZAWA, S., LU, H., SAMANT, S., LIU, C., LI, J. G. \& PALTA, J. R. 2011. An image quality comparison study between XVI and OBI CBCT systems. Journal of Applied Clinical Medical Physics, 12.

KAN, M. W. K., LEUNG, L. H. T., WONG, W. \& LAM, N. 2008. Radiation dose from cone beam computed tomography for image-guided radiation therapy. International Journal of Radiation Oncology* Biology* Physics, 70, 272-279.

KAPANEN, M., COLLAN, J., BEULE, A., SEPPÄLÄ, T., SAARILAHTI, K. \& TENHUNEN, M. 2013. Commissioning of MRI - only based treatment planning procedure for external beam radiotherapy of prostate. Magnetic resonance in medicine, $70,127-135$.

KAPANEN, M. \& TENHUNEN, M. 2013. T1/T2*-weighted MRI provides clinically relevant pseudo-CT density data for the pelvic bones in MRI-only based radiotherapy treatment planning. Acta Oncologica, 52, 612-618.

KAROTKI, A., MAH, K., MEIJER, G. \& MELTSNER, M. 2011. Comparison of bulk electron density and voxel-based electron density treatment planning. Journal of Applied Clinical Medical Physics, 12.

KAWRAKOW, I. 2001. VMC++, electron and photon Monte Carlo calculations optimized for radiation treatment planning. Advanced Monte Carlo for Radiation Physics, Particle Transport Simulation and Applications. Springer. 
KAWRAKOW, I. \& ROGERS, D. W. O. 2000. The EGSnrc code system. NRC Report PIRS-701, NRC, Ottawa.

KEALL, P. J. 1996. Electron transport in photon and election beam modelling. KEEREMAN, V., FIERENS, Y., BROUX, T., DE DEENE, Y., LONNEUX, M.\& VANDENBERGHE, S. 2010. MRI-based attenuation correction for PET/MRI using ultrashort echo time sequences. Journal of nuclear medicine, 51, 812818.

KEEREMAN, V., VANHOVE, C.\& VANDENBERGHE, S. 2012. MRI - Based Attenuation Correction for Emission Tomography using Ultrashort Echo Time Sequences. eMagRes.

KHAN, F. M. \& STATHAKIS, S. 2010. The Physics of Radiation Therapy. Medical Physics, 37.

KNOLL, G. F. 2010. Radiation detection and measurement, John Wiley \& Sons. KNÖÖS, T., WIESLANDER, E., COZZI, L., BRINK, C., FOGLIATA, A., ALBERS, D., NYSTRÖM, H. \& LASSEN, S. 2006. Comparison of dose calculation algorithms for treatment planning in external photon beam therapy for clinical situations. Physics in medicine and biology, 51, 5785.

KOIVULA, L., WEE, L. \& KORHONEN, J. 2016. Feasibility of MRI-only treatment planning for proton therapy in brain and prostate cancers: Dose calculation accuracy in substitute CT images. Medical Physics, 43, 4634-4642.

KORHONEN, J. 2015. Magnetic resonance imaging-based radiation therapy-Methods enabling the radiation therapy treatment planning workflow for prostate cancer patients by relying solely on MRI-based images throughout the process.

KORHONEN, J., KAPANEN, M., KEYRILÄINEN, J., SEPPÄLÄ, T. \& TENHUNEN, M. 2014. A dual model HU conversion from MRI intensity values within and outside of bone segment for MRI-based radiotherapy treatment planning of prostate cancer. Medical physics, 41, 011704.

KRIEGER, T. \& SAUER, O. A. 2005. Monte Carlo-versus pencil-beam-/collapsedcone-dose calculation in a heterogeneous multi-layer phantom. Physics in medicine and biology, 50, 859.

KUFE, D. W., POLLOCK, R. E., WEICHSELBAUM, R. R., BAST JR, R. C., GANSLER, T. S., HOLLAND, J. F. \& FREI III, E. 2003. Holland-Frei cancer medicine. 
KUTCHER, G. J. \& BURMAN, C. 1989. Calculation of complication probability factors for non-uniform normal tissue irradiation: The effective volume method gerald. International Journal of Radiation Oncology* Biology* Physics, 16, 1623-1630.

LA, S., CEBERG, C., TOMASZEWICZ, A. \& WEBER, L. 2009. Conversion of greyscale intensity values from CBCT images acquired on Elekta XVI to HU. Radiotherapy and Oncology, 92, S64.

LAGENDIJK, J. J. W., RAAYMAKERS, B. W., VAN DEN BERG, C. A. T., MOERLAND, M. A., PHILIPPENS, M. E. \& VAN VULPEN, M. 2014a. MR guidance in radiotherapy. Physics in medicine and biology, 59, R349.

LAGENDIJK, J. J. W., RAAYMAKERS, B. W. \& VAN VULPEN, M. The magnetic resonance imaging-linac system. Seminars in radiation oncology, 2014b. Elsevier, 207-209.

LAGENDIJK, J. J. W., VULPEN, M. \& RAAYMAKERS, B. W. 2016. The development of the MRI linac system for online MRI - guided radiotherapy: a clinical update. Journal of internal medicine.

LAMBERT, J., GREER, P. B., MENK, F., PATTERSON, J., PARKER, J., DAHL, K., GUPTA, S., CAPP, A., WRATTEN, C. \& TANG, C. 2011. MRI-guided prostate radiation therapy planning: Investigation of dosimetric accuracy of MRI-based dose planning. Radiotherapy and Oncology, 98, 330-334.

LANGEN, K. M. \& JONES, D. T. L. 2001. Organ motion and its management. International Journal of Radiation Oncology* Biology* Physics, 50, 265-278.

LEE, C., LANGEN, K. M., LU, W., HAIMERL, J., SCHNARR, E., RUCHALA, K. J., OLIVERA, G. H., MEEKS, S. L., KUPELIAN, P. A. \& SHELLENBERGER, T. D. 2008a. Evaluation of geometric changes of parotid glands during head and neck cancer radiotherapy using daily MVCT and automatic deformable registration. Radiotherapy and Oncology, 89, 81-88.

LEE, L., LE, Q.-T. \& XING, L. 2008b. Retrospective IMRT dose reconstruction based on cone-beam CT and MLC log-file. International Journal of Radiation Oncology* Biology* Physics, 70, 634-644.

LÉTOURNEAU, D., MARTINEZ, A. A., LOCKMAN, D., YAN, D., VARGAS, C., IVALDI, G. \& WONG, J. 2005a. Assessment of residual error for online 
cone-beam CT-guided treatment of prostate cancer patients. International Journal of Radiation Oncology* Biology* Physics, 62, 1239-1246.

LÉTOURNEAU, D., WONG, J. W., OLDHAM, M., GULAM, M., WATT, L., JAFFRAY, D. A., SIEWERDSEN, J. H. \& MARTINEZ, A. A. 2005b. Conebeam-CT guided radiation therapy: technical implementation. Radiotherapy and Oncology, 75, 279-286.

LI, J., YAO, W., XIAO, Y.\& YU, Y. 2013. Feasibility of improving cone-beam CT number consistency using a scatter correction algorithm. Journal of Applied Clinical Medical Physics, 14.

LI, T., SCHREIBMANN, E., YANG, Y.\& XING, L. 2005. Motion correction for improved target localization with on-board cone-beam computed tomography. Physics in medicine and biology, 51, 253.

LI, T., ZHU, X., THONGPHIEW, D., LEE, W. R., VUJASKOVIC, Z., WU, Q., YIN, F.-F. \& WU, Q. J. 2010. On-line adaptive radiation therapy: feasibility and clinical study. Journal of oncology, 2010.

LI, X. A. 2011. Adaptive radiation therapy, CRC Press.

LI, X. A., ALBER, M., DEASY, J. O., JACKSON, A., JEE, K.-W. K., MARKS, L. B., MARTEL, M. K., MAYO, C., MOISEENKO, V.\& NAHUM, A. E. 2012. The use and QA of biologically related models for treatment planning: Short report of the TG-166 of the therapy physics committee of the AAPM a). Medical Physics, 39, 1386-1409.

LIN, S.-Y., CHU, T.-C. \& LIN, J.-P. 2001. Monte Carlo simulation of a clinical linear accelerator. Applied Radiation and Isotopes, 55, 759-765.

LIU, B., BENNETT, J., WANG, G., DE MAN, B., ZENG, K., YIN, Z., FITZGERALD, P.\& YU, H. 2012. Completeness map evaluation demonstrated with candidate next - generation cardiac CT architectures. Medical physics, 39, 2405-2416.

LOW, D. A., HARMS, W. B., MUTIC, S. \& PURDY, J. A. 1998. A technique for the quantitative evaluation of dose distributions. Medical physics, 25, 656-661.

LU, B., LU, H. \& PALTA, J. 2010. A comprehensive study on decreasing the kilovoltage cone-beam CT dose by reducing the projection number. Journal of Applied Clinical Medical Physics, 11. 
LYMAN, J. T. 1985. Complication probability as assessed from dose-volume histograms. Radiation Research, 104, S13-S19.

MACKIE, T. R., BIELAJEW, A. F., ROGERS, D. W. O. \& BATTISTA, J. J. 1988. Generation of photon energy deposition kernels using the EGS Monte Carlo code. Physics in medicine and biology, 33, 1 .

MACKIE, T. R., EL - KHATIB, E., BATTISTA, J., SCRIMGER, J., VAN DYK, J. \& CUNNINGHAM, J. R. 1985a. Lung dose corrections for 6 - and 15 - MV x rays. Medical physics, 12, 327-332.

MACKIE, T. R., RECKWERDT, P., MCNUTT, T., GEHRING, M. \& SANDERS, C. 1996. Photon beam dose computations. Teletherapy: Present and Future, 103135.

MACKIE, T. R., SCRIMGER, J. W. \& BATTISTA, J. J. 1985b. A convolution method of calculating dose for 15 - MV x rays. Medical physics, 12, 188-196.

MAIL, N., MOSELEY, D. J., SIEWERDSEN, J. H. \& JAFFRAY, D. A. 2008. An empirical method for lag correction in cone-beam CT. Medical physics, 35, 5187-5196.

MAIL, N., MOSELEY, D. J., SIEWERDSEN, J. H. \& JAFFRAY, D. A. 2009. The influence of bowtie filtration on cone-beam CT image quality. Medical Physics, 36, 22-32.

MALTZ, J. S., BLANZ, W.-E., HRISTOV, D. \& BANI-HASHEMI, A. Cone beam $\mathrm{X}$-ray scatter removal via image frequency modulation and filtering. Engineering in Medicine and Biology Society, 2005. 1854-1857.

MARCHANT, T. E., MOORE, C. J., ROWBOTTOM, C. G., MACKAY, R. I. \& WILLIAMS, P. C. 2008. Shading correction algorithm for improvement of cone-beam CT images in radiotherapy. Physics in medicine and biology, 53, 5719 .

MARTIN, J. M., FRANTZIS, J., EADE, T. \& CHUNG, P. 2010. Clinician's guide to prostate IMRT plan assessment and optimisation. Journal of medical imaging and radiation oncology, 54, 569-575.

MAUND, I. F., BENSON, R. J., FAIRFOUL, J., COOK, J., HUDDART, R. \& POYNTER, A. 2014. Image-guided radiotherapy of the prostate using daily CBCT: the feasibility and likely benefit of implementing a margin reduction. The British journal of radiology, 87, 20140459. 
MAYLES, P. 2007. Handbook of Radiotherapy Physics.

MAYLES, P., NAHUM, A. \& ROSENWALD, J.-C. 2007. Handbook of radiotherapy physics: theory and practice, CRC Press.

MAYLES, P., NAHUM, A. \& ROSENWALD, J.-C. 2010. Handbook of radiotherapy physics: theory and practice, CRC Press.

MCDERMOTT, P. N. 2016. Tutorials in Radiotherapy Physics: Advanced Topics with Problems and Solutions, CRC Press LLC.

METCALFE, P. E., WONG, T. P. \& HOBAN, P. W. 1993. Radiotherapy X-ray beam inhomogeneity corrections: the problem of lateral electronic disequilibrium in lung. Australasian physical \& engineering sciences in medicine/supported by the Australasian College of Physical Scientists in Medicine and the Australasian Association of Physical Sciences in Medicine, 16, 155-167.

METROPOLIS, N. 1987. The beginning of the Monte Carlo method. Los Alamos Science, 15, 125-130.

METROPOLIS, N. \& ULAM, S. 1949. The monte carlo method. Journal of the American statistical association, 44, 335-341.

MICHALSKI, J. M., GAY, H., JACKSON, A., TUCKER, S. L. \& DEASY, J. O. 2010. Radiation dose-volume effects in radiation-induced rectal injury. International Journal of Radiation Oncology* Biology* Physics, 76, S123S129.

MILAN, J. \& BENTLEY, R. E. 1974. The storage and manipulation of radiation dose data in a small digital computer. The British journal of radiology, 47, 115-121.

MOHAN, R., MAGERAS, G. S., BALDWIN, B., BREWSTER, L. J., KUTCHER, G. J., LEIBEL, S., BURMAN, C. M., LING, C. C. \& FUKS, Z. 1992. Clinically relevant optimization of 3 - D conformal treatments. Medical physics, 19, 933-944.

MØLLER, C. 1932. Passage of hard beta rays through matter. Ann. Physik, 14, 531. MORGAN-FLETCHER, S. L. 2001. Prescribing, recording and reporting photon beam therapy (Supplement to ICRU Report 50), ICRU Report 62. ICRU, pp. ix+ 52, 1999 (ICRU Bethesda, MD) \$65.00 ISBN 0-913394-61-0. The British Journal of Radiology, 74, 294-294.

MORIN, O., CHEN, J., AUBIN, M., GILLIS, A., AUBRY, J.-F., BOSE, S., CHEN, H., DESCOVICH, M., XIA, P. \& POULIOT, J. 2007. Dose calculation using 
megavoltage cone-beam CT. International Journal of Radiation Oncology* Biology* Physics, 67, 1201-1210.

MOSELEY, D. J., WHITE, E. A., WILTSHIRE, K. L., ROSEWALL, T., SHARPE, M. B., SIEWERDSEN, J. H., BISSONNETTE, J.-P., GOSPODAROWICZ, M., WARDE, P. \& CATTON, C. N. 2007. Comparison of localization performance with implanted fiducial markers and cone-beam computed tomography for on-line image-guided radiotherapy of the prostate.

International Journal of Radiation Oncology* Biology* Physics, 67, 942-953.

MURPHY, M. J. 2011. Adaptive motion compensation in radiotherapy, Boca Raton, FL, USA, CRC Press.

MURTHY, V., MASTER, Z., ADURKAR, P., MALLICK, I., MAHANTSHETTY, U., BAKSHI, G., TONGAONKAR, H. \& SHRIVASTAVA, S. 2011. 'Plan of the day'adaptive radiotherapy for bladder cancer using helical tomotherapy. Radiotherapy and Oncology, 99, 55-60.

MUTIC, S. \& DEMPSEY, J. F. The ViewRay system: magnetic resonance-guided and controlled radiotherapy. Seminars in radiation oncology, 2014. Elsevier, 196-199.

NELSON, W. R. \& HIRAYAMA, H. 1985. DW 0. Rogers, “The EGS4 Code System”. Stanford Linear Accelerator Center report SLAC-265.

NEUENSCHWANDER, H., MACKIE, T. R. \& RECKWERDT, P. J. 1995. MMC-a high-performance Monte Carlo code for electron beam treatment planning. Physics in medicine and biology, 40, 543.

NIJKAMP, J., POS, F. J., NUVER, T. T., DE JONG, R., REMEIJER, P., SONKE, J.J. \& LEBESQUE, J. V. 2008. Adaptive radiotherapy for prostate cancer using kilovoltage cone-beam computed tomography: first clinical results. International Journal of Radiation Oncology* Biology* Physics, 70, 75-82.

NING, R., TANG, X. \& CONOVER, D. 2004. X-ray scatter correction algorithm for cone beam CT imaging. Medical physics, 31, 1195-1202.

NISBET, A., BEANGE, I., VOLLMAR, H.-S., IRVINE, C., MORGAN, A. \& THWAITES, D. I. 2004. Dosimetric verification of a commercial collapsed cone algorithm in simulated clinical situations. Radiotherapy and oncology, $73,79-88$. 
NUVER, T. T., HOOGEMAN, M. S., REMEIJER, P., VAN HERK, M. \&

LEBESQUE, J. V. 2007. An adaptive off-line procedure for radiotherapy of prostate cancer. International Journal of Radiation Oncology* Biology* Physics, 67, 1559-1567.

O'CONNOR, J. E. 1957. The variation of scattered x-rays with density in an irradiated body. Physics in medicine and biology, 1, 352.

OBORN, B. M., METCALFE, P. E., BUTSON, M. J. \& ROSENFELD, A. B. 2010. Monte Carlo characterization of skin doses in $6 \mathrm{MV}$ transverse field MRIlinac systems: effect of field size, surface orientation, magnetic field strength, and exit bolus. Medical physics, 37, 5208-5217.

OELKFE, U. \& SCHOLZ, C. 2006. Dose calculation algorithms. New Technologies in Radiation Oncology. Springer.

OLDHAM, M., LÉTOURNEAU, D., WATT, L., HUGO, G., YAN, D., LOCKMAN, D., KIM, L. H., CHEN, P. Y., MARTINEZ, A. \& WONG, J. W. 2005. Conebeam-CT guided radiation therapy: A model for on-line application. Radiotherapy and oncology, 75, 271. E1-271. E8.

ONOZATO, Y., KADOYA, N., FUJITA, Y., ARAI, K., DOBASHI, S., TAKEDA, K., KISHI, K., UMEZAWA, R., MATSUSHITA, H. \& JINGU, K. 2014. Evaluation of On-Board kV Cone Beam Computed Tomography-Based Dose Calculation With Deformable Image Registration Using Hounsfield Unit Modifications. International Journal of Radiation Oncology* Biology* Physics, 89, 416-423.

ONOZATO, Y., KADOYA, N., FUJITA, Y., KATSUTA, Y., DOBASHI, S., TAKEDA, K., KISHI, K., SATOU, K., ABE, K. \& UMEZAWA, R. Evaluation of dose calculation accuracy of modified CBCT using Multi-levelthreshold algorithm. World Congress on Medical Physics and Biomedical Engineering May 26-31, 2012, Beijing, China, 2013. Springer, 1799-1802. OWEN, R., KRON, T., FOROUDI, F., MILNER, A., COX, J., DUCHESNE, G., CLEEVE, L., ZHU, L., CRAMB, J. \& SPARKS, L. 2009. Comparison of CT on rails with electronic portal imaging for positioning of prostate cancer patients with implanted fiducial markers. International Journal of Radiation Oncology* Biology* Physics, 74, 906-912. 
PAPANIKOLAOU, N., BATTISTA, J. J., BOYER, A. L., KAPPAS, C., KLEIN, E., MACKIE, T. R., SHARPE, M. \& VAN DYK, J. 2004. Tissue inhomogeneity corrections for megavoltage photon beams. AAPM Task Group, 65, 1-142.

PAUWELS, R., STAMATAKIS, H., BOSMANS, H., BOGAERTS, R., JACOBS, R., HORNER, K. \& TSIKLAKIS, K. 2013. Quantification of metal artifacts on cone beam computed tomography images. Clinical oral implants research, 24, 94-99.

PAWLOWSKI, J. M., YANG, E. S., MALCOLM, A. W., COFFEY, C. W. \& DING, G. X. 2010. Reduction of dose delivered to organs at risk in prostate cancer patients via image-guided radiation therapy. International Journal of Radiation Oncology* Biology* Physics, 76, 924-934.

PEARSON, E. A., CHO, S., PELIZZARI, C. A. \& PAN, X. Non-circular cone beam CT trajectories: A preliminary investigation on a clinical scanner. Nuclear Science Symposium Conference Record (NSS/MIC), 2010 IEEE, 2010. IEEE, 3172-3175.

PETKOVSKA, S., TOLEVSKA, C., KRALEVA, S. \& PETRESKA, E. Conformity index for brain cancer patients. Conference on medical physics and biomedical engineering, 2010. 56.

PILIERO, M. 2014. Modelling and development of tissue-equivalent dosimeters for small field radiotherapy. $\mathrm{PhD}$ Thesis, Swansea University

PINEDA, A. R., SIEWERDSEN, J. H. \& TWARD, D. J. Analysis of image noise in 3D cone-beam CT: Spatial and Fourier domain approaches under conditions of varying stationarity. Medical Imaging, 2008. International Society for Optics and Photonics, 69131Q-69131Q-10.

PISANI, L., LOCKMAN, D., JAFFRAY, D., YAN, D., MARTINEZ, A. \& WONG, J. 2000. Setup error in radiotherapy: on-line correction using electronic kilovoltage and megavoltage radiographs. International Journal of Radiation Oncology* Biology* Physics, 47, 825-839.

POLUDNIOWSKI, G. G., EVANS, P. M. \& WEBB, S. 2012. Cone beam computed tomography number errors and consequences for radiotherapy planning: an investigation of correction methods. International Journal of Radiation Oncology* Biology* Physics, 84, e109-e114. 
PRELL, D., KYRIAKOU, Y. \& KALENDER, W . A. 2009. Comparison of ring artifact correction methods for flat-detector CT. Physics in medicine and biology, 54, 3881 .

PURDIE, T. G., BISSONNETTE, J.-P., FRANKS, K., BEZJAK, A., PAYNE, D., SIE, F., SHARPE, M. B. \& JAFFRAY, D. A. 2007. Cone-beam computed tomography for on-line image guidance of lung stereotactic radiotherapy: localization, verification, and intrafraction tumor position. International Journal of Radiation Oncology* Biology* Physics, 68, 243-252.

RAAIJMAKERS, A. J. E., HÅRDEMARK, B., RAAYMAKERS, B. W., RAAIJMAKERS, C. P. J. \& LAGENDIJK, J. J. W. 2007. Dose optimization for the MRI-accelerator: IMRT in the presence of a magnetic field. Physics in medicine and biology, 52, 7045.

RAAIJMAKERS, A. J. E., RAAYMAKERS, B. W. \& LAGENDIJK, J. J. W. 2005. Integrating a MRI scanner with a $6 \mathrm{MV}$ radiotherapy accelerator: dose increase at tissue-air interfaces in a lateral magnetic field due to returning electrons. Physics in medicine and biology, 50, 1363.

RAAYMAKERS, B. W., RAAIJMAKERS, A. J. E., KOTTE, A., JETTE, D. \& LAGENDIJK, J. J. W. 2004. Integrating a MRI scanner with a 6 MV radiotherapy accelerator: dose deposition in a transverse magnetic field. Physics in medicine and biology, 49, 4109.

RAFIC, M. \& RAVINDRAN, P. 2015. Evaluation of on-board imager cone beam CT hounsfield units for treatment planning using rigid image registration. Journal of cancer research and therapeutics, 11, 690.

RANK, C. M., TREMMEL, C., HÜNEMOHR, N., NAGEL, A. M., JÄKEL, O.\& GREILICH, S. 2013. MRI-based treatment plan simulation and adaptation for ion radiotherapy using a classification-based approach. Radiation Oncology, 8, 1.

RECKWERDT, P. J. \& MACKIE, T. R. 1992. Superposition/convolution speed improvements using Run-Length Raytracing. Med.Phys, 19, 784.

RICHTER, A., HU, Q., STEGLICH, D., BAIER, K., WILBERT, J., GUCKENBERGER, M.\& FLENTJE, M. 2008. Investigation of the usability of conebeam CT data sets for dose calculation. Radiat Oncol, 3, 42. 
RINKEL, J., GERFAULT, L., ESTEVE, F. \& DINTEN, J. M. 2007. A new method for X-ray scatter correction: first assessment on a cone-beam CT experimental setup. Physics in medicine and biology, 52, 4633.

ROESKE, J. C., FORMAN, J. D., MESINA, C. F., HE, T., PELIZZARI, C. A., FONTENLA, E., VIJAYAKUMAR, S. \& CHEN, G. T. Y. 1995. Evaluation of changes in the size and location of the prostate, seminal vesicles, bladder, and rectum during a course of external beam radiation therapy. International Journal of Radiation Oncology* Biology* Physics, 33, 1321-1329.

ROGERS, D. W. O. \& BIELAJEW, A. F. 1988. Experimental benchmarks of EGS. Monte Carlo Transport of Electrons and Photons. Springer.

ROGERS, D. W. O. \& BIELAJEW, A. F. 1990. The Dosimetry of Ionizing Radiation. 3.

ROGERS, D. W. O., FADDEGON, B. A., DING, G. X., MA, C. M., WE, J. \& MACKIE, T. R. 1995. BEAM: A Monte Carlo code to simulate radiotherapy treatment units. Medical physics, 22, 503-524.

ROGERS, D. W. O., WALTERS, B. \& KAWRAKOW, I. 2001. BEAMnrc users manual. NRC Report PIRS, 509.

RUBINSTEIN, A. E., GUINDANI, M. \& HAZLE, J. D. 2014. Investigating magnetic field dose effects in small animals: a Monte Carlo study. International Journal of Cancer Therapy and Oncology, 2.

SCHLEGEL, W. C., BRADY, L. W., HEILMANN, H. P., BORTFELD, T., MOLLS, M. \& GROSU, A. L. 2006. New technologies in radiation oncology, Springer.

SCHMIDT, M. A. \& PAYNE, G. S. 2015. Radiotherapy planning using MRI. Physics in medicine and biology, 60, R323.

SCHNEIDER, U., PEDRONI, E. \& LOMAX, A. 1996. The calibration of CT Hounsfield units for radiotherapy treatment planning. Physics in medicine and biology, 41, 111.

SCHULZE, R., HEIL, U., GRO B , D., BRUELLMANN, D. D., DRANISCHNIKOW, E., SCHWANECKE, U. \& SCHOEMER, E. 2014. Artefacts in CBCT: a review. Dentomaxillofacial Radiology.

SEABY, A. W., THOMAS, D. W., RYDE, S. J. S., LEY, G. R. \& HOLMES, D. 2002. Design of a multiblock phantom for radiotherapy dosimetry applications. The British journal of radiology, 75, 56-58. 
SECO, J. \& VERHAEGEN, F. 2013. Monte Carlo techniques in radiation therapy, CRC Press.

SHAW, C. C. 2014. Cone beam computed tomography, Taylor \& Francis.

SHEIKH-BAGHERI, D. \& ROGERS, D. W. O. 2002. Monte Carlo calculation of nine megavoltage photon beam spectra using the BEAM code. Medical physics, 29, 391-402.

SHOWALTER, T. N., NAWAZ, A. O., XIAO, Y., GALVIN, J. M. \& VALICENTI, R. K. 2008. A cone beam CT-based study for clinical target definition using pelvic anatomy during postprostatectomy radiotherapy. International Journal of Radiation Oncology* Biology* Physics, 70, 431-436.

SIEWERDSEN, J. H. \& JAFFRAY, D. A. 2000. Optimization of x-ray imaging geometry (with specific application to flat-panel cone-beam computed tomography). Medical physics, 27, 1903-1914.

SIEWERDSEN, J. H., MOSELEY, D. J. \& JAFFRAY, D. A. Incorporation of task in 3D imaging performance evaluation: The impact of asymmetric NPS on detectability. Medical Imaging 2004, 2004. International Society for Optics and Photonics, 89-97.

SMITH, B. D. 1985. Image reconstruction from cone-beam projections: necessary and sufficient conditions and reconstruction methods. Medical Imaging, IEEE Transactions on, 4, 14-25.

SMITSMANS, M. H. P., DE BOIS, J., SONKE, J.-J., BETGEN, A., ZIJP, L. J., JAFFRAY, D. A., LEBESQUE, J. V. \& VAN HERK, M. 2005. Automatic prostate localization on cone-beam CT scans for high precision image-guided radiotherapy. International Journal of Radiation Oncology* Biology* Physics, 63, 975-984.

SMITSMANS, M. H. P., POS, F. J., DE BOIS, J., HEEMSBERGEN, W. D., SONKE, J.-J., LEBESQUE, J. V . \& VAN HERK, M. 2008. The influence of a dietary protocol on cone beam CT-guided radiotherapy for prostate cancer patients. International Journal of Radiation Oncology* Biology* Physics, 71, 1279-1286.

SON, J., BAEK, T., LEE, B., SHIN, D., PARK, S. Y., PARK, J., LIM, Y. K., LEE, S. B., KIM, J. \& YOON, M. 2015. A comparison of the quality assurance of four 
dosimetric tools for intensity modulated radiation therapy. Radiology and oncology, 49, 307-313.

SONG, P. Y., WASHINGTON, M., VAIDA, F., HAMILTON, R., SPELBRING, D., WYMAN, B., HARRISON, J., CHEN, G. T. Y. \& VIJAYAKUMAR, S. 1996. A comparison of four patient immobilization devices in the treatment of prostate cancer patients with three dimensional conformal radiotherapy. International Journal of Radiation Oncology* Biology* Physics, 34, 213-219. SONKE, J.-J., ZIJP, L., REMEIJER, P. \& VAN HERK, M. 2005. Respiratory correlated cone beam CT. Medical physics, 32, 1176-1186.

SONTAG, M. R. \& CUNNINGHAM, J. R. 1978. The Equivalent Tissue-Air Ratio Method for Making Absorbed Dose Calculations in a Heterogeneous Medium 1. Radiology, 129, 787-794.

SPEZI, E., DOWNES, P., JARVIS, R., RADU, E. \& STAFFURTH, J. 2012. Patientspecific three-dimensional concomitant dose from cone beam computed tomography exposure in image-guided radiotherapy. International Journal of Radiation Oncology* Biology* Physics, 83, 419-426.

SPEZI, E., LEWIS, D. G. \& SMITH, C. W. 2002. A DICOM-RT-based toolbox for the evaluation and verification of radiotherapy plans. Physics in medicine and biology, 47, 4223.

SPIRYDOVICH, S., PAPIEZ, L., LANGER, M., SANDISON, G. \& THAI, V. 2006. High density dental materials and radiotherapy planning: Comparison of the dose predictions using superposition algorithm and fluence map Monte Carlo method with radiochromic film measurements. Radiotherapy and oncology, 81, 309-314.

SRINIVASAN, K. 2015. Applications of cone beam computed tomography in radiotherapy treatment planning.

STANESCU, T., TADIC, T. \& JAFFRAY, D. A. 2014. Commissioning of an MRguided radiation therapy system. International Journal of Radiation Oncology* Biology* Physics, 90, S94-S95.

STILlIE, A. L., KRON, T., FOX, C., HERSCHTAL, A., HAWORTH, A., THOMPSON, A., OWEN, R., TAI, K. H., DUCHESNE, G. \& FOROUDI, F. 2009. Rectal filling at planning does not predict stability of the prostate gland 
during a course of radical radiotherapy if patients with large rectal filling are re-imaged. Clinical Oncology, 21, 760-767.

STORM, L. \& ISRAEL, H. I. 1970. Photon cross sections from $1 \mathrm{keV}$ to $100 \mathrm{MeV}$ for elements $\mathrm{Z}=1$ to $\mathrm{Z}=100$. Atomic Data and Nuclear Data Tables, 7, 565681.

SVEISTRUP, J., AF ROSENSCHÖLD, P. M., DEASY, J. O., OH, J. H., POMMER, T., PETERSEN, P. M. \& ENGELHOLM, S. A. 2014. Improvement in toxicity in high risk prostate cancer patients treated with image-guided intensitymodulated radiotherapy compared to 3D conformal radiotherapy without daily image guidance. Radiation Oncology, 9, 1 .

SYKES, J. R., LINDSAY, R., IBALL, G. \& THWAITES, D. I. Dosimetry of CBCT: methods, doses and clinical consequences. Journal of Physics: Conference Series, 2013. IOP Publishing, 012017.

TAKEMURA, A., TANABE, S., TOKAI, M., UEDA, S., NOTO, K., ISOMURA, N. \& KOJIMA, H. 2015. Long-term stability of the Hounsfield unit to electron density calibration curve in cone-beam computed tomography images for adaptive radiotherapy treatment planning. Journal of Radiotherapy in Practice, 14, 410-417.

TANG, X., NING, R., YU, R. \& CONOVER, D. 2001. Cone beam volume CT image artifacts caused by defective cells in x-ray flat panel imagers and the artifact removal using a wavelet-analysis-based algorithm. Medical physics, 28, 812825 .

THOR, M., PETERSEN, J. B. B., BENTZEN, L., HØYER, M. \& MUREN, L. P. 2011. Deformable image registration for contour propagation from CT to cone-beam CT scans in radiotherapy of prostate cancer. Acta Oncologica, 50, 918-925.

THORNTON, S. T. \& REX, A. 2012. Modern physics for scientists and engineers, Cengage Learning.

TURBELL, H. 2001. Cone-beam reconstruction using filtered backprojection.

TUY, H. K. 1983. An inversion formula for cone-beam reconstruction. SIAM Journal on Applied Mathematics, 43, 546-552. 
VAN ZIJTVELD, M., DIRKX, M. \& HEIJMEN, B. 2007. Correction of conebeam CT values using a planning CT for derivation of the "dose of the day". Radiotherapy and Oncology, 85, 195-200.

VERHAEGEN, F. \& SEUNTJENS, J. 2003. Monte Carlo modelling of external radiotherapy photon beams. Physics in medicine and biology, 48, R107.

WALTERS, B., KAWRAKOW, I. \& ROGERS, D. W. O. 2005. DOSXYZnrc users manual. NRC Report PIRS, 794.

WANG, J. \& XING, L. Accurate determination of the shape and location of metal objects in X-ray computed tomography. SPIE Medical Imaging, 2010. International Society for Optics and Photonics, 76225A-76225A-10.

WEBER, D. C., WANG, H., ALBRECHT, S., OZSAHIN, M., TKACHUK, E., ROUZAUD, M., NOUET, P. \& DIPASQUALE, G. 2008. Open low-field magnetic resonance imaging for target definition, dose calculations and set-up verification during three-dimensional CRT for glioblastoma multiforme. Clinical Oncology, 20, 157-167.

WIESLANDER, E. \& KNÖÖS, T. 2000. A virtual linear accelerator for verification of treatment planning systems. Physics in medicine and biology, 45, 2887.

WILLIAMSON, J. F., WHITING, B. R., BENAC, J., MURPHY, R. J., BLAINE, G. J., O’SUlliVAN, J. A., POLITTE, D. G. \& SNYDER, D. L. 2002. Prospects for quantitative computed tomography imaging in the presence of foreign metal bodies using statistical image reconstruction. Medical physics, 29, 24042418.

WILSON, R. R. 1951. The range and straggling of high energy electrons. Physical Review, 84, 100.

WONG, J. W., JAFFRAY, D. A., SIEWERDSEN, J. H. \& YAN, D. 2014. Cone beam CT: Transforming radiation treatment guidance, planning, and monitoring. Cone Beam Computed Tomography, 223.

WOO, M. K. \& CUNNINGHAM, J. R. 1990. The validity of the density scaling method in primary electron transport for photon and electron beams. Medical physics, 17, 187-194.

WU, Q. J., THONGPHIEW, D., WANG, Z., MATHAYOMCHAN, B., CHANKONG, V., YOO, S., LEE, W. R. \& YIN, F.-F. 2008. On-line reoptimization of prostate IMRT plans for adaptive radiation therapyAbstract 
and preliminary data presented at 49th AAPM Annual Meeting, Minneapolis, MN, USA, July 2007. Physics in medicine and biology, 53, 673.

YANG, Y., SCHREIBMANN, E., LI, T., WANG, C. \& XING, L. 2007. Evaluation of on-board kV cone beam CT (CBCT)-based dose calculation. Physics in medicine and biology, 52, 685.

YOO, S. \& YIN, F.-F. 2006. Dosimetric feasibility of cone-beam CT-based treatment planning compared to CT-based treatment planning. International Journal of Radiation Oncology* Biology* Physics, 66, 1553-1561.

ZELEFSKY, M. J., PEI, X., CHOU, J. F., SCHECHTER, M., KOLLMEIER, M., COX, B., YAMADA, Y., FIDALEO, A., SPERLING, D. \& HAPPERSETT, L. 2011. Dose escalation for prostate cancer radiotherapy: predictors of longterm biochemical tumor control and distant metastases-free survival outcomes. European urology, 60, 1133-1139.

ZHU, L., WANG, J. \& XING, L. 2009. Noise suppression in scatter correction for cone-beam CT. Medical physics, 36, 741-752. 